\title{
Advanced Materials for Radiation Dosimetry
}

by

\section{Christin Gädtke}

\author{
A thesis \\ submitted to Victoria University of Wellington \\ in fulfilment of the requirements for the degree of \\ Doctor of Philosophy \\ in Physics.
}

Victoria University of Wellington

2014 



\section{Abstract}

The motivation for this work was to find materials that have the following characteristics: good optical transparency, rapid read-out, automation of read-out, good fading characteristic, promise high sensitivity to ionising radiation, and tissue equivalence for use in medical applications. For example, there are medical applications in brachytherapy and high-energy photon therapy for the treatment of cancer. These applications benefit small dosimeters for monitoring radiation during radiotherapy or for dose verification and validation.

This thesis studies fluoroperovskite materials that were manufactured as bulk materials or nanoparticles. The techniques of photoluminescence (PL), radioluminescence (RL), thermoluminescence (TL) and optically stimulated luminescence (OSL) were employed in order to get a deeper understanding of the defect distribution in these materials. A detailed model of the trap distribution was developed from the results of these measurements. It was observed that compared to the bulk materials, the nanoparticles show a lower PL lifetime and less dependence on the dose of the RL intensity, which is due to the different defect distribution. The nanoparticles also demonstrate more low temperature peaks in the TL glow curves. PL and RL measurements of $\mathrm{Eu}^{3+}$ doped samples show that the crystal environment of the $\mathrm{Eu}^{3+}$ in the bulk material is more distorted than for the nanoparticles. For the bulk materials, the thermal coefficient of the RL is $<0.4 \% / \mathrm{K}$, which is a desirable property of real -time dosimeters. The thermal coefficient of the RL in the nanoparticles has a high uncertainty $(\sim 7 \% / \mathrm{K})$ compared to the bulk materials $(\sim 0.4 \% / \mathrm{K})$. For the fluoride nanoparticles, it was observed that the PL lifetimes for the $\mathrm{LaF}_{3}$ decreases with increasing rare earth concentrations. This can be attributed to energy transfer from luminescence ions in the core to luminescence ions near the surface followed by non-radiative decay. In comparison, the decrease of the PL lifetimes of $\mathrm{RbMgF}_{3}$ and $\mathrm{NaMgF}_{3}$ is predominantly due to non-radiative recombination centres inside the crystal. 



\section{Acknowledgments}

The results presented in this thesis were part of my three years work at the photonics group at Callaghan Innovation (former Industrial Research Ltd.) and at the radiation imaging and detection group at Victoria University of Wellington.

I would like to take the opportunity to thank everyone that made the completion of this thesis possible.

First of all, I would like to express my sincere gratitude to my day-to-day supervisor Dr. Grant Williams for his support, guidance and invaluable discussions throughout my $\mathrm{PhD}$. In similar way I would like to thank my co-supervisor Dr. Andy Edgar.

I also would like to thank everyone belonging to the photonics group of Callaghan Innovation for their assistance, in particular David Clarke for preparing and also assisting in the preparation of the nanoparticles and Sebastiampillai Raymond for his help with the long-term RL measurements. Stefaan Janssens for his help with some of the measurements and interesting discussions.

At Callaghan Innovation, I would like to thank Martin Ryan for his help with the XRD. I would like to thank Nicola Winch and Laura Dixie from VUW for their assistance with the fluorescence spectrometer and the thermoluminescence spectrometer and Chris Varoy for the help with the preparation of the bulk polycrystalline materials.

I would like to acknowledge the funding I received from the MacDiarmid Institute.

Finally, I would like to thank my mum, Heike Gädtke, and my friends for their support throughout these years. 



\section{Contents}

List of Figures $\quad$ vi

List of Tables $\quad$ xxiii

\begin{tabular}{lll}
\hline 1 & Introduction & 1
\end{tabular}

2 Basic quantities and units in radiation dosimetry 3

2.1 Radiometric quantities . . . . . . . . . . . . . . . . . . 11

$2.2 \quad$ Interaction of radiation with matter and their energy dependence . . . . . 11

2.2 .1 Interaction of radiation with matter . . . . . . . . . . . . . . . . . 11

$2.2 .2 \quad$ Energy dependence of a radiative process . . . . . . . . . . . . 13

$2.2 .3 \quad$ Effective atomic number $\ldots \ldots \ldots \ldots$. . . . . . . . . . . . 17

2.2 .4 Radiation protection dosimetry . . . . . . . . . . . . . . . 18

2.3 Dosimetric quantities . . . . . . . . . . . . . . . . . . . . . . . . . . . 18

$2.4 \quad$ Fields of dosimetry $\ldots \ldots \ldots \ldots$. . . . . . . . . . . . . . . . . . 19

2.4 .1 Personal dosimetry $\ldots \ldots \ldots \ldots$

2.4 .2 Medical dosimetry . . . . . . . . . . . . . . . . . 20

2.4 .3 Environmental dosimetry . . . . . . . . . . . . . . . . . 24

2.4 .4 Retrospective dosimetry . . . . . . . . . . . . . . . . . 24

\begin{tabular}{|lll}
\hline 3 & Theoretical Background & 25
\end{tabular}

3.1 Fluoroperovskite $\ldots \ldots \ldots \ldots$. . . . . . . . . . . . . . . . . . . . . . 25

3.2 Photoluminescence . . . . . . . . . . . . . . . . . . . . . . . . 26 
$3.2 .1 \quad$ Photoluminescence of $\mathrm{Eu}^{2+}$ in fluoroperovskites . . . . . . . . . . 28

$3.2 .2 \quad$ Photoluminescence of $\mathrm{Mn}^{2+}$ in fluoroperovskites . . . . . . . . . . 30

3.2 .3 Judd-Ofelt theory $\ldots \ldots \ldots$. . . . . . . . . . . . . . . . . . 32

3.2 .4 Photoluminescence lifetime measurements . . . . . . . . . . . . . . 33

3.3 Thermoluminescence $\ldots \ldots \ldots \ldots$. . . . . . . . . . . . . . . . . . . . . 34

3.4 Radioluminescence . . . . . . . . . . . . . . . . . . . . . 36

$3.5 \quad$ Optically Stimulated Luminescence $\ldots \ldots \ldots$

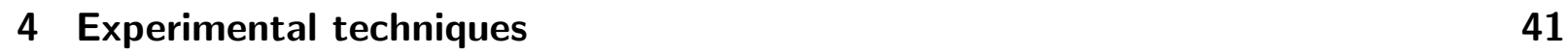

4.1 Sample preparation . . . . . . . . . . . . . . . . . . . 41

$4.1 .1 \quad$ Polycrystalline samples . . . . . . . . . . . . . . . . . . . . . . 41

4.1 .2 Nanoparticles . . . . . . . . . . . . . . . . . . . . . . . . . 42

4.2 X-ray diffraction $\ldots \ldots \ldots \ldots$. . . . . . . . . . . . . . . . . . . . . . . .

4.3 Thermoluminescence measurements . . . . . . . . . . . . . . . . . . 45

4.4 Radioluminescence measurements . . . . . . . . . . . . . . . . . . . . . . . . 46

4.5 Radiation facilities $\ldots \ldots \ldots$. . . . . . . . . . . . . . . . . . . . . . . . . . . 47

4.6 Photoluminescence, PL lifetime and temperature dependent PL measurements 48

5 Bulk polycrystalline $\mathrm{NaMgF}_{3}$

5.1 Photoluminescence and PL lifetime measurements . . . . . . . . . . . . . 52

$5.1 .1 \quad$ Eu doped polycrystalline $\mathrm{NaMgF}_{3} \ldots \ldots \ldots \ldots$. . . . . . . . . . 52

$5.1 .2 \quad$ Mn doped polycrystalline $\mathrm{NaMgF}_{3} \ldots \ldots \ldots$. . . . . . . . 60

5.2 Thermoluminescence measurements . . . . . . . . . . . . . . . . . . 64

$5.2 .1 \quad$ Eu doped polycrystalline $\mathrm{NaMgF}_{3} \ldots \ldots \ldots \ldots$. . . . . . . . . 64

$5.2 .2 \quad$ Mn doped polycrystalline $\mathrm{NaMgF}_{3} \ldots \ldots \ldots$. . . . . . . . 67

5.3 Radioluminescence and temperature dependent RL measurements . . . . . 73

5.3 .1 Radioluminescence measurements . . . . . . . . . . . . . . . . . . . 74

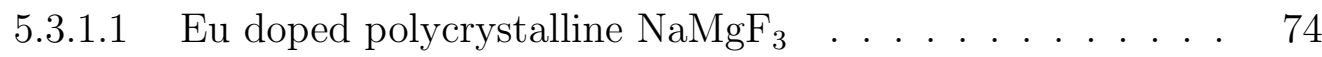

$5.3 .1 .2 \quad$ Mn doped polycrystalline $\mathrm{NaMgF}_{3}$. . . . . . . . . . . 77 
5.3 .2 Temperature dependent RL measurements $\ldots \ldots \ldots \ldots$. . . 80

$\begin{array}{llll}5.3 .2 .1 & \text { Eu doped polycrystalline } \mathrm{NaMgF}_{3} & \ldots \ldots \ldots \ldots & \ldots\end{array}$

5.3 .2 .2 Mn doped polycrystalline $\mathrm{NaMgF}_{3} \ldots \ldots \ldots \ldots$

5.4 Optically stimulated luminescence measurements $\ldots \ldots \ldots$. . . . . 86

5.5 Conclusion $\ldots \ldots \ldots \ldots$. . . . . . . . . . . . . . . . . . . . . . . . . . . 88

\begin{tabular}{lll}
\hline & $\mathrm{NaMgF}_{3}$ nanoparticles & 91
\end{tabular}

6.1 Photoluminescence and PL lifetime measurements . . . . . . . . . . . 91

$6.1 .1 \quad$ Eu doped $\mathrm{NaMgF}_{3}$ nanoparticles $\ldots \ldots \ldots \ldots \ldots \ldots \ldots$

6.1 .2 Mn doped $\mathrm{NaMgF}_{3}$ nanoparticles . . . . . . . . . . . . 102

6.2 Thermoluminescence measurements . . . . . . . . . . . . . . . . . . . 106

6.2 .1 Eu doped $\mathrm{NaMgF}_{3}$ nanoparticles $\ldots \ldots \ldots \ldots \ldots$

$6.2 .2 \quad$ Mn doped $\mathrm{NaMgF}_{3}$ nanoparticles $\ldots \ldots \ldots \ldots \ldots \ldots$

6.3 Radioluminescence and temperature dependent RL measurements . . . . 111

6.3 .1 Radioluminescence measurements . . . . . . . . . . . . . . . . 111

6.3 .1 .1 Eu doped $\mathrm{NaMgF}_{3}$ nanoparticles $\ldots \ldots \ldots \ldots \ldots \ldots$

$6.3 .1 .2 \quad$ Mn doped $\mathrm{NaMgF}_{3}$ nanoparticles $\ldots \ldots \ldots \ldots$

6.3 .2 Temperature dependent RL measurements . . . . . . . . . . . . 119

$6.3 .2 .1 \quad$ Eu doped $\mathrm{NaMgF}_{3}$ nanoparticles $\ldots \ldots \ldots \ldots \ldots$

$6.3 .2 .2 \quad$ Mn doped $\mathrm{NaMgF}_{3}$ nanoparticles $\ldots \ldots \ldots \ldots \ldots$

6.4 Conclusion . . . . . . . . . . . . . . . . . . . . . . . . . . . . . . . . . . . . . . . . . . . . 124

$\begin{array}{lll}7 & \text { Bulk polycrystalline } \mathrm{RbMgF}_{3} & 127\end{array}$

7.1 Photoluminescence and PL lifetime measurements . . . . . . . . . . . . . 127

$7.1 .1 \quad$ Eu doped polycrystalline $\mathrm{RbMgF}_{3} \ldots \ldots \ldots \ldots \ldots \ldots$

7.1 .2 Mn doped polycrystalline $\mathrm{RbMgF}_{3} \quad \ldots \ldots \ldots \ldots \ldots$

7.2 Thermoluminescence measurements . . . . . . . . . . . . . . . . . . . 134

$7.2 .1 \quad$ Eu doped polycrystalline $\mathrm{RbMgF}_{3} \ldots \ldots \ldots \ldots \ldots \ldots$

7.2 .2 Mn doped polycrystalline $\mathrm{RbMgF}_{3} \quad \ldots \ldots \ldots \ldots \ldots$ 
$7.3 \quad$ Radioluminescence and temperature dependent RL measurements . . . . 139

7.3 .1 Radioluminescence measurements . . . . . . . . . . . . . . . . . 139

\begin{tabular}{llllll}
\hline 7.3 .1 .1 & Eu doped polycrystalline $\mathrm{RbMgF}_{3}$ & $\ldots$ & $\ldots$
\end{tabular}$\ldots \ldots \ldots$

7.3.1.2 Mn doped polycrystalline $\mathrm{RbMgF}_{3} \ldots \ldots \ldots \ldots$

7.3 .2 Temperature dependent RL measurements . . . . . . . . . . . . . 144

7.3.2.1 Eu doped polycrystalline $\mathrm{RbMgF}_{3} \ldots \ldots \ldots \ldots$

$7.3 .2 .2 \quad$ Mn doped polycrystalline $\mathrm{RbMgF}_{3} \ldots \ldots \ldots \ldots$

7.4 Conclusion . . . . . . . . . . . . . . . . . . . . . . . . . . . . 147

$\begin{array}{lll}8 & \mathrm{RbMgF}_{3} \text { nanoparticles } & 149\end{array}$

8.1 Photoluminescence and PL lifetime measurements . . . . . . . . . . . . . . 149

8.1 .1 Eu doped $\mathrm{RbMgF}_{3}$ nanoparticles . . . . . . . . . . . . . . . . . . 149

$8.1 .2 \quad$ Mn doped $\mathrm{RbMgF}_{3}$ nanoparticles $\ldots \ldots \ldots \ldots \ldots$

8.2 Thermoluminescence measurements . . . . . . . . . . . . . . . . . . 167

$8.2 .1 \quad$ Eu doped $\mathrm{RbMgF}_{3}$ nanoparticles $\ldots \ldots \ldots \ldots \ldots$

$8.2 .2 \quad$ Mn doped $\mathrm{RbMgF}_{3}$ nanoparticles $\ldots \ldots \ldots \ldots \ldots$

8.3 Radioluminescence and temperature dependent RL measurements . . . . . 171

8.3 .1 Radioluminescence measurements . . . . . . . . . . . . . . . . . 172

8.3.1.1 Eu doped $\mathrm{RbMgF}_{3}$ nanoparticles $\ldots \ldots \ldots \ldots \ldots$

8.3 .1 .2 Mn doped $\mathrm{RbMgF}_{3}$ nanoparticles $\ldots \ldots \ldots \ldots$

8.3 .2 Temperature dependent RL measurements $\ldots \ldots \ldots \ldots$

$8.3 .2 .1 \quad$ Eu doped $\mathrm{RbMgF}_{3}$ nanoparticles $\ldots \ldots \ldots \ldots$. . . . . 179

$8.3 .2 .2 \quad$ Mn doped $\mathrm{RbMgF}_{3}$ nanoparticles $\ldots \ldots \ldots \ldots \ldots$

8.4 Conclusion . . . . . . . . . . . . . . . . . . . . . . . 186

$\begin{array}{lll}9 & \text { Other polycrystalline nanoparticles } & 189\end{array}$

9.1 Photoluminescence and PL lifetime measurements of $\mathrm{LaF}_{3} \ldots \ldots$

$9.1 .1 \quad$ Eu doped $\mathrm{LaF}_{3}$ nanoparticles $\ldots \ldots \ldots \ldots \ldots \ldots$

$9.1 .2 \quad$ Sm doped $\mathrm{LaF}_{3}$ nanoparticles $\ldots \ldots \ldots \ldots \ldots \ldots$ 
9.2 Radioluminescence measurements of $\mathrm{LaF}_{3} \quad \ldots \ldots \ldots$

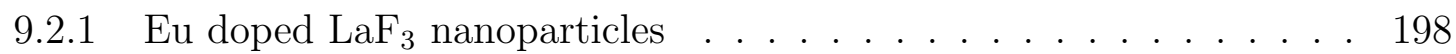

$9.2 .2 \quad$ Sm doped $\mathrm{LaF}_{3}$ nanoparticles . . . . . . . . . . . . . . . . . . . . 199

9.3 Photoluminescence measurements of $\mathrm{BaF}_{2} \quad \ldots \ldots \ldots$. . . . . . . . . . . . . 200

9.4 Radioluminescence measurements of $\mathrm{BaF}_{2} \quad \ldots \ldots \ldots$. . . . . . . . . . . . 201

9.5 Photoluminescence measurements of $\mathrm{BaMgF}_{4} \ldots \ldots$. . . . . . . . . . . . 202

9.6 Radioluminescence measurements of $\mathrm{BaMgF}_{4} \quad \ldots \ldots \ldots$

9.7 Conclusion . . . . . . . . . . . . . . . . . . . . . . . . . . . . . . . 204

$\begin{array}{ll}10 \text { Summary and Conclusion } & 207\end{array}$

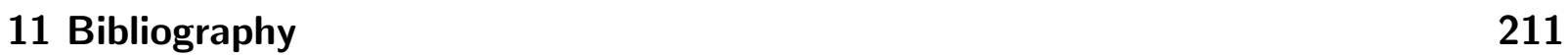

12 List of publications 223 


\section{List of Figures}

2.1 Graph of the Rayleigh, photoelectric, Compton, pair production, and total mass attenuation coefficient for soft tissue $(Z=7)$ as a function of energy [1]. 10

2.2 Unrestricted $\mathrm{S} / \rho$ and restricted $\left((\mathrm{L} / \rho)_{\Delta}\right.$ with $\Delta=10$ and $\left.100 \mathrm{keV}\right)$ total mass stopping powers for carbon $\left(\rho=1.70 \mathrm{~g} / \mathrm{cm}^{3}\right)$. Vertical lines indicate the points at which restricted and unrestricted mass stopping powers begin to diverge as the kinetic energy increases [2] . . . . . . . . . . . . . . . . . 13

2.3 Mass energy absorption coefficients for detector materials. . . . . . . . . . 14

$2.4 \quad$ Energy response of detector materials compared to tissue. . . . . . . . . . . 15

2.5 Burlin cavity theory parameter $d$ as a function of photon energy for two cylindrical plastic scintillator sizes. This parameter represents the fraction of the dose deposited in the cavity due to electrons generated outside the cavity [1]. . . . . . . . . . . . . . . . . . . . . . . . . . . . 16

3.1 (a) Orthorhombic structure of $\mathrm{NaMgF}_{3}$ and (b) Hexagonal structure of

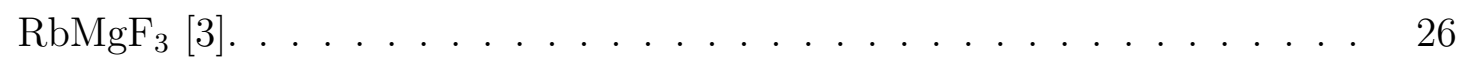

3.2 Photoluminescence process. $\ldots \ldots \ldots \ldots$. . . . . . . . . . . . . . . 27

3.3 Configuration coordinate diagram of $\mathrm{Eu}^{2+}$ in $\mathrm{RbMgF}_{3}$ [4]. . . . . . . . . . 30

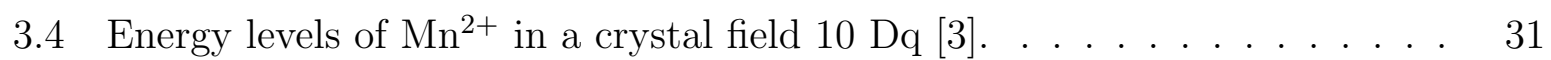

3.5 One-trap/ one- centre model for the TL process [5]. . . . . . . . . . . . . . 35

3.6 Model of the one trap, one recombination centre for RL. . . . . . . . . . . 37

3.7 One-trap/ one-centre OSL model [6]. . . . . . . . . . . . . . . . . . . . . . 39 
4.1 XRD diffractogram of $\operatorname{RbMgF}_{3}(\mathrm{a})$ and $\mathrm{NaMgF}_{3}(\mathrm{~b})$ bulks and the reference pattern for $\mathrm{RbMgF}_{3}$ (blue lines in (a)) and $\mathrm{NaMgF}_{3}$ (red lines in (b)). XRD diffractogram of $\mathrm{RbMgF}_{3}(\mathrm{c})$ and $\mathrm{NaMgF}_{3}(\mathrm{~d})$ nanoparticles and the reference

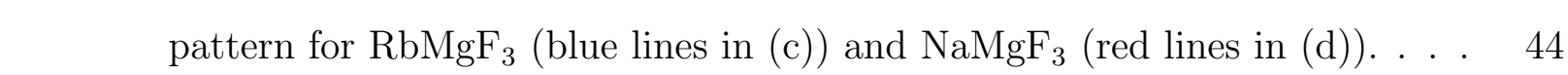

4.2 Schematic of the thermoluminescence measurements. . . . . . . . . . . . 46

4.3 Schematic of the radioluminescence measurements with the ${ }^{241} \mathrm{Am}$-source. . $\quad 47$

4.4 Schematic configuration of the Varian Cary Eclipse fluorescence spectrometer[7]. 49

4.5 Schematic configuration of the Jobin-Yvon FluoroLog $\left.{ }^{\mathrm{R}}\right)$ spectrometer [8]. . $\quad 50$

5.1 (a) PL excitation (dashed curve, $\lambda_{e m}=360$ (blue) $\mathrm{nm}$ and $420 \mathrm{~nm}$ (black)) \begin{tabular}{|l|l|}
\hline and emission (solid curve, $\lambda_{e x}=256 \mathrm{~nm}$ ) spectra for $\mathrm{NaMgF}_{3} 0.2 \%$ Eu. (b) \\
\hline Configuration coordinate diagram of $\mathrm{Eu}^{2+}$ in $\mathrm{NaMgF}_{3}[\underline{3},[9]$. . . . . . . . . & 53
\end{tabular}

5.2 (a) Absolute PL intensity of the emission spectra, $\lambda_{e x}=256 \mathrm{~nm}$, for $\mathrm{NaMgF}_{3}$ \begin{tabular}{|c|c|}
\hline $0.2 \%$ Eu before (solid curve) and after irradiation with a dose of $~ 19 \mathrm{kGy}$ \\
\hline
\end{tabular} (dashed curve). Inset: Normalised PL of the emission spectra, $\lambda_{e x}=256 \mathrm{~nm}$, \begin{tabular}{|c|}
\hline for $\mathrm{NaMgF}_{3} 0.2 \%$ Eu before (solid curve) and after irradiation with a dose \\
\hline of $\sim 19 \mathrm{kGy}$ (dashed curve) (b) PL excitation at $\lambda_{e m}=610 \mathrm{~nm}$ (blue: solid
\end{tabular} of $\sim 19 \mathrm{kGy}$ (dashed curve). (b) PL excitation at $\lambda_{e m}=610 \mathrm{~nm}$ (blue: solid \begin{tabular}{|c|}
\hline curve - before irradiation, dashed curve - after irradiation with a dose of $\sim 19$ \\
\hline
\end{tabular} \begin{tabular}{|c|c|}
\hline $\mathrm{kGy}$ ) and emission at $\lambda_{e x}=396 \mathrm{~nm}$ (red: solid curve - before irradiation, \\
\hline
\end{tabular}

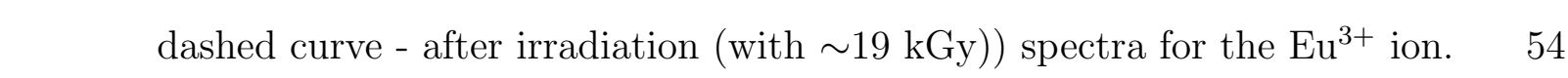

5.3 (a) Absolute PL intensity of the excitation spectra, $\lambda_{e m}=610 \mathrm{~nm}$, before (solid curve) and after irradiation with a dose of $\sim 19$ kGy (dashed curve). \begin{tabular}{|c|}
\hline (b) Absolute PL intensity of the emission spectra, $\lambda_{e x}=396 \mathrm{~nm}$, before (solid \\
\hline curve) and after irradiation with a dose of $\sim 19 \mathrm{kGy}$ (dashed curve) (c) PL \\
\hline
\end{tabular} \begin{tabular}{|c|}
\hline emission spectra of $\mathrm{Eu}^{3+}$ corrected for the broad unknown peak before (solid \\
\hline
\end{tabular} curve) and after heavy irradiation with a dose of $\sim 19$ kGy (dashed curve).

(d) Absolute PL excitation spectra at $\lambda_{e m}=450 \mathrm{~nm}$ before (solid curve) and after heavy irradiation with a dose of $\sim 19 \mathrm{kGy}$ (dashed curve). . . . . . . . 56 
5.4 (a) Change of the crystal field leading to a shift in the 5d4f level in $\mathrm{Eu}^{2+}$.

(b) Electron from $\mathrm{Eu}^{2+}$ recombines in a deep non-optical or non-thermal trap. 57

5.5 (a) PL lifetime decay of $0.2 \%$ Eu doped $\mathrm{NaMgF}_{3}$ at $\lambda_{e m}=360 \mathrm{~nm}$ (solid curve) and (b) $\lambda_{e m}=390 \mathrm{~nm}$ emission (solid curve). The dashed curve is the exponential fit. Both lifetimes were measured at $\lambda_{e x}=256 \mathrm{~nm}$. . . . . 58

5.6 (a) PL excitation spectrum at $\lambda_{e m}=500 \mathrm{~nm}$ (dashed curve) and $\lambda_{e m}=600$ $\mathrm{nm}$ emission (solid curve) for the $1 \% \mathrm{Mn}$ doped $\mathrm{NaMgF}_{3}$ sample. (b) PL emission spectrum for $\mathrm{NaMgF}_{3}$ doped with $0.5 \% \mathrm{Mn}$ (dashed curve) and $1 \% \mathrm{Mn}$ (solid curve) excited at $396 \mathrm{~nm}$. (c) Broadening of the peaks in the PL excitation spectra for the distorted $\mathrm{Mn}^{2+}$ site. (d) Splitting of the energy levels of $\mathrm{Mn}^{2+}$ in an octahedral crystal field. . . . . . . . . . .

5.7 (a) PL lifetime decay of $1 \% \mathrm{Mn}$ doped $\mathrm{NaMgF}_{3}$ at $\lambda_{e m}=500 \mathrm{~nm}$ (solid curve) and (b) $\lambda_{e m}=600 \mathrm{~nm}$ emission (solid curve). The dashed curve is the exponential fit. Both lifetimes were measured at $\lambda_{e x}=465 \mathrm{~nm}$.

5.8 (a) TL for the $\mathrm{NaMgF}_{3}$ sample doped with $0.2 \% \mathrm{Eu}$ (solid curve). The TL \begin{tabular}{|c|}
\hline data was detected with the CCD after 5 min X-ray irradiation $(40 \mathrm{kV}, 40$ \\
\hline $\mathrm{mA}$ ) at a heating rate of $1 \mathrm{~K} / \mathrm{s}$. The fitted peaks were obtained using first \\
\hline order kinetics glow curve deconvolution (dashed curves). (b) TL spectra
\end{tabular} vs. wavelength for the different temperatures. (c) Model for the probability of the emission from the $4 \mathrm{f}$ and $5 \mathrm{~d}$ levels to the ground states for higher temperatures. . . . . . . . . . . . . . . . .

5.9 (a) TL for the $\mathrm{NaMgF}_{3}$ sample doped with $0.5 \% \mathrm{Mn}$ (solid curve). The TL data was detected with the PMT after 1 min X-ray irradiation $(40 \mathrm{kV}, 40$ $\mathrm{mA}$ ) at a heating rate of $1 \mathrm{~K} / \mathrm{s}$. The fitted peaks were obtained using first order kinetics glow curve deconvolution (dashed curves). (b) TL spectra vs. wavelength for the different temperatures detected after 5 min of Xray irradiation $(40 \mathrm{kV}, 40 \mathrm{~mA})$ detected with the CCD. (c) Normalized TL intensity of the TL curves at $443 \mathrm{~K}$ (blue curve) and $622 \mathrm{~K}$ (black curve). (d) Model for F-centre recombination close to the regular $\mathrm{Mn}^{2+}$ site. . . . 68 
5.10 TL for the $\mathrm{NaMgF}_{3}$ sample doped with $1 \% \mathrm{Mn}$ (solid curve). The TL data was detected with the PMT after 1 min (a), repeated after another 5 min (b) and repeated again after 1 min (c) of X-ray irradiation $(40 \mathrm{kV}, 40$ \begin{tabular}{|c|}
\hline $\mathrm{mA}$ ) at a heating rate of $1 \mathrm{~K} / \mathrm{s}$. The fitted peaks were obtained using first \\
\hline
\end{tabular} \begin{tabular}{|c|}
\hline order kinetics glow curve deconvolution (dashed curves). (d) TL spectra \\
\hline
\end{tabular} vs. wavelength for the different temperatures detected after 5 min of X-ray

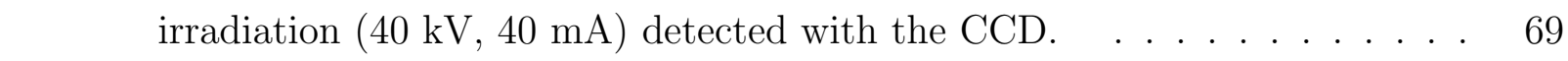

5.11 (a) TL for the $\mathrm{NaMgF}_{3}$ sample doped with $1 \% \mathrm{Mn}$ (solid curve). The TL data was detected with the PMT after 1 min, 5 min and again 1 min X-ray irradiation. (b) Normalized TL intensity of the TL curves at $427 \mathrm{~K}$ (blue curve) and $622 \mathrm{~K}$ (black curve). . . . . . . . . . . . . . 71

5.12 TL for $\mathrm{NaMgF}_{3}: 1 \% \mathrm{Mn}$ after 1 min irradiation and $2 \mathrm{~s}$ of bleaching with

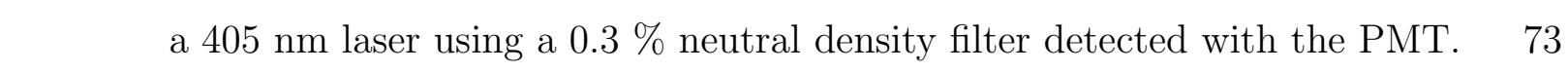

5.13 (a) Radioluminescence spectra for $\mathrm{NaMgF}_{3}: 0.2 \%$ Eu for different doses and \begin{tabular}{|c|}
\hline (b) the integrated RL spectrum as a function of dose during continuous X- \\
\hline ray irradiation $\left(\mathrm{Eu}^{2+}\right.$ filled squares and $\mathrm{Eu}^{3+}$ open squares). (c) Existence \\
\hline of electron and hole traps next to the Eu. (d) Dose dependence of the RL \\
\hline signal as a result of the generation of non-radiative recombination centres. \\
\hline
\end{tabular}

5.14 (a) Radioluminescence spectra for $\mathrm{NaMgF}_{3}: 0.5 \%$ Mn for different doses \begin{tabular}{|c|}
\hline and (b) the integrated RL spectra as a function of dose during continuous X- \\
\hline ray irradiation (regular $\mathrm{Mn}^{2+}$ site filled squares and highly distorted $\mathrm{Mn}^{2+}$ \\
\hline
\end{tabular}

\begin{tabular}{|l|}
\hline site open squares). (c) Radioluminescence spectra for $\mathrm{NaMgF}_{3}: 1 \% \mathrm{Mn}$ \\
\hline for different doses and (d) the integrated RL spectra as a function of dose \\
\hline during continuous X-ray irradiation (regular $\mathrm{Mn}^{2+}$ site filled squares and \\
\hline highly distorted $\mathrm{Mn}^{2+}$ site open squares). . . . . . . . . . . . . . . . 78
\end{tabular}


5.15 Normalised RL spectra for low doses (blue curve) and after 12 kGy irradi\begin{tabular}{|l|}
\hline ation (black curve) for $0.5 \%$ (a) and $1 \% \mathrm{Mn}$ (b) doped sample. Ratio of \\
\hline the RL intensity of the distorted peak divided by the RL intensity of the \\
\hline regular peak as a function of dose for $0.5 \% \mathrm{Mn}(\mathrm{c})$ and $1 \% \mathrm{Mn}(\mathrm{d})$ doped \\
\hline
\end{tabular}

5.16 (a) Temperature dependent RL for $\mathrm{NaMgF}_{3}: 0.2 \% \mathrm{Eu}$ for different temperature up to $100{ }^{\circ} \mathrm{C}$. Arrows facing up signal an increase of temperature, \begin{tabular}{|c|}
\hline and arrows facing down signal a decrease in temperature. (b) RL spectra \\
\hline
\end{tabular} \begin{tabular}{|c|}
\hline measured for different temperatures. (c) Model of the RL and TL trapping \\
\hline
\end{tabular} and detrapping in $\mathrm{NaMgF}_{3}$ doped with Eu. . . . . . . . . . . . . . . 82

5.17 (a) Temperature dependent RL for $0.5 \% \mathrm{Mn}$ and (b) for $1 \% \mathrm{Mn}$ doped $\mathrm{NaMgF}_{3}$ for different temperature up to $100{ }^{\circ} \mathrm{C}$. Blue indicates the regular \begin{tabular}{|c|}
\hline $\mathrm{Mn}^{2+}$ site and red the highly distorted site. (c) Total RL signal of the \\
\hline
\end{tabular} \begin{tabular}{|c|}
\hline distorted and the regular site versus the temperature for the $1 \%$ doped \\
\hline sample (top graph) and the $0.5 \%$ sample (bottom graph). (d) RL spectra \\
\hline
\end{tabular} sample (top graph) and the $0.5 \%$ sample (bottom graph). (d) RL spectra

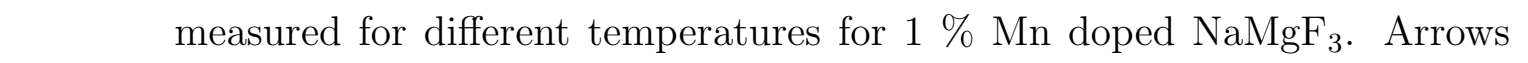
facing up signal an increase of temperature, and arrows facing down signal a decrease in temperature. Black curves show the trend of the temperature

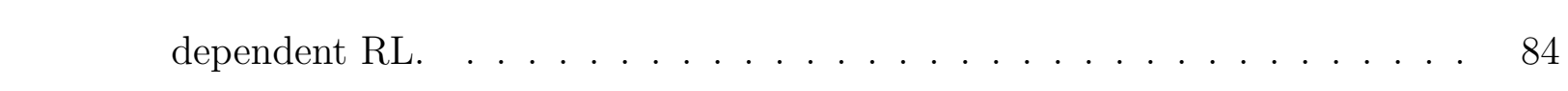

5.18 (a) Absolute OSL signal versus time for the different doses. (b) The to\begin{tabular}{|c|}
\hline tal OSL signal plotted versus the total dose. (c) The change of the OSL \\
\hline
\end{tabular} signal ( $\triangle$ OSL) divided by the change of dose versus the total dose. (d) \begin{tabular}{|l}
\hline OSL $/ \Delta$ dose versus the change of the dose $(\Delta$ dose $)$. \\
\hline
\end{tabular}$\ldots \ldots$. . . . 87

$6.1 \quad$ (a) PL excitation for the $\mathrm{Eu}^{2+}\left(\lambda_{e m}=360 \mathrm{~nm}\right.$ - dashed curve $)$ and $\mathrm{Eu}^{3+}$ \begin{tabular}{|c|}
\hline$\left(\lambda_{e m}=592 \mathrm{~nm}\right.$ - solid curve) and (b) emission $\left(\lambda_{e x}=396 \mathrm{~nm}\right.$; inset $\lambda_{e x}=256$ \\
\hline $\mathrm{nm})$ spectra for $\mathrm{NaMgF}_{3}$ doped with $1 \% \mathrm{Eu}$ (dashed curve) and $5 \% \mathrm{Eu}$ \\
\hline
\end{tabular} (solid curve). . . . . . . . . . . . . . . . . . 92 
6.2 (a) PL emission spectra for $0.1 \%$, (b) $0.5 \%$. (c) $1 \%$ and (d) $5 \%$ Eu doped $\mathrm{NaMgF}_{3}$ at $\lambda_{e x}=396 \mathrm{~nm}$ at $10 \mathrm{~K}$ (solid curve) and at room temperature (dashed curve). Inset: PL excitation spectra detected at $\lambda_{e m}=450 \mathrm{~nm}$ for the different Eu concentrations of $\mathrm{NaMgF}_{3}$.

6.3 (a) Temperature dependent average PL lifetime of $1 \%$ Eu doped $\mathrm{NaMgF}_{3}$ $\left(\lambda_{e m}=592 \mathrm{~nm} ; \lambda_{e x}=375 \mathrm{~nm}\right)$ from the three exponential fit of the data with only the $\tau_{1}$ (filled squares), $\tau_{2}$ (filled circles), $\tau_{3}$ (filled triangles) and the average lifetime, $\tau_{123, \text { ave }}$ (open squares). $\tau_{3}$ was fitted using equation 6.1 . (b) Temperature dependent average PL lifetime of $1 \%$ Eu doped $\mathrm{NaMgF}_{3}$ from \begin{tabular}{|c|}
\hline the three exponential fit of the data with only the $\tau_{1}$ (filled squares), $\tau_{2}$ (filled \\
\hline
\end{tabular} circles) and the average lifetime, $\tau_{12, a v e}$ (open squares) when only using the first and the second component. . . . . . . . . . . . . . . . . . 96

6.4 (a) Temperature dependent average PL lifetime of $5 \%$ Eu doped $\mathrm{NaMgF}_{3}$ \begin{tabular}{|c|}
\hline$\left(\lambda_{e m}=592 \mathrm{~nm} ; \lambda_{e x}=375 \mathrm{~nm}\right)$ from the three exponential fit of the data \\
\hline
\end{tabular} \begin{tabular}{|c|}
\hline with only the $\tau_{1}$ (filled squares), $\tau_{2}$ (filled circles), $\tau_{3}$ (filled triangles) and the \\
\hline
\end{tabular} \begin{tabular}{|c|c|c|}
\hline average lifetime, $\tau_{123, \text { ave }}$ (open squares). $\tau_{3}$ was fitted using equation 6.1 . (b) \\
\hline
\end{tabular} Temperature dependent average PL lifetime of $5 \% \mathrm{Eu}$ doped $\mathrm{NaMgF}_{3}$ from the three exponential fit of the data with only the $\tau_{1}$ (filled squares), $\tau_{2}$ (filled circles) and the average lifetime, $\tau_{12, a v e}$ (open squares) when only using the first and the second component. . . . . . . . . . . . . . . . 97

6.5 (a) Average PL lifetime for $\mathrm{Eu}^{3+}\left(\lambda_{e m}=592 \mathrm{~nm}, \lambda_{e x}=375 \mathrm{~nm}\right)$ doped $\mathrm{NaMgF}_{3}$ nanoparticles against the rare earth concentration. (b) The PL lifetime for the unknown broad peak was measured at $\lambda_{e m}=500 \mathrm{~nm}$ and

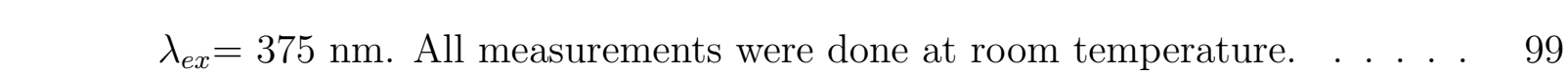

$6.6 \quad$ (a) PL lifetime decay of $5 \% \mathrm{Eu}$ doped $\mathrm{NaMgF}_{3}$ at $\lambda_{e m}=590 \mathrm{~nm}$ (solid \begin{tabular}{|c|}
\hline curve). The dashed curve is the exponential fit. The lifetime was measured \\
\hline
\end{tabular} \begin{tabular}{|c|c|}
\hline at $\lambda_{e x}=375 \mathrm{~nm}$. (b) The lifetime decay curves at $\lambda_{e m}=590 \mathrm{~nm}$ emission \\
\hline
\end{tabular} for the different Eu concentrations in $\mathrm{NaMgF}_{3} . \ldots \ldots$. . . . . . . . . . 100 
6.7 (a) PL excitation spectra for $5 \% \mathrm{Mn}$ doped $\mathrm{NaMgF}_{3}$ at $\lambda_{e m}=500 \mathrm{~nm}$ (dashed curve) and $\lambda_{e m}=600 \mathrm{~nm}$ (solid curve) for the distorted $\mathrm{Mn}^{2+}$ site. \begin{tabular}{|c|}
\hline (b) PL emission spectrum for $\mathrm{NaMgF}_{3}$ doped with $1 \% \mathrm{Mn}$ (red), $2 \%$ (blue) \\
\hline
\end{tabular} and $5 \%$ Mn (black) excited at $396 \mathrm{~nm} . \quad \ldots \ldots$. . . . . . . . . 103

6.8 (a) PL lifetime decay of $1 \% \mathrm{Mn}$ doped $\mathrm{NaMgF}_{3}$ at $\lambda_{e m}=500 \mathrm{~nm}$ (solid curve) and (b) $\lambda_{e m}=600 \mathrm{~nm}$ (solid curve). The dashed curve is the exponential fit.

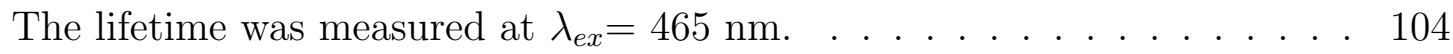

6.9 Average PL lifetime for the distorted $\mathrm{Mn}^{2+}$ site $\left(\lambda_{e m}=500 \mathrm{~nm}\right.$ red squares $)$ \begin{tabular}{|c|}
\hline and the regular $\mathrm{Mn}^{2+}$ site $\left(\lambda_{e m}=600 \mathrm{~nm}\right.$ blue squares $)$ doped $\mathrm{NaMgF}_{3}$ \\
\hline
\end{tabular} nanoparticles versus the rare earth concentration. . . . . . . . . . . . . 105

6.10 TL glow curves for $\mathrm{NaMgF}_{3}$ doped with $0.5 \%$ (a) and $5 \%$ (b) Eu after 20 min irradiation with the X-ray generator operating at $40 \mathrm{kV}$ and $40 \mathrm{~mA}$ (solid curves). The TL was detected with the PMT. The fitted peaks were

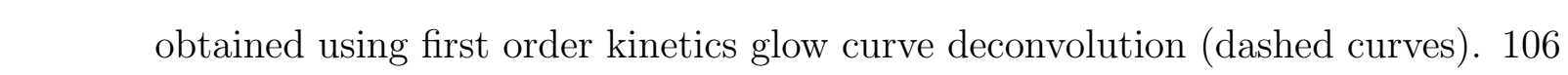

6.11 TL glow curves for $\mathrm{NaMgF}_{3}$ doped with $1 \% \mathrm{Mn}(\mathrm{a}), 2 \% \mathrm{Mn}$ (b), $5 \% \mathrm{Mn}$ \begin{tabular}{|c|}
\hline (c) after $20 \mathrm{~min}$ irradiation and for $1 \% \mathrm{Mn}$ after $10 \mathrm{~min}, 30 \mathrm{~min}$ and again \\
\hline
\end{tabular} $10 \mathrm{~min}$ (d) irradiation with the X-ray generator operating at $40 \mathrm{kV}$ and 40 \begin{tabular}{|c|}
\hline mA (solid curves). The TL was detected with the PMT. The fitted peaks \\
\hline
\end{tabular} \begin{tabular}{|c|}
\hline were obtained using first order kinetics glow curve deconvolution (dashed \\
\hline
\end{tabular} curves.$\quad \ldots \ldots \ldots \ldots$

6.12 Radioluminescence spectra for $\mathrm{NaMgF}_{3}$ nanoparticles doped with $0.1 \% \mathrm{Eu}$ \begin{tabular}{|c|}
\hline (a) and $0.5 \% \mathrm{Eu}(\mathrm{c})$ for different doses and the integrated RL spectra as a \\
\hline function of dose during continuous X-ray irradiation for $0.1 \% \mathrm{Eu}(\mathrm{b})$ and \\
\hline $0.5 \% \mathrm{Eu}(\mathrm{d})$ doped samples $\left(\mathrm{Eu}^{2+}\right.$ open squares and $\mathrm{Eu}^{3+}$ filled squares). \\
\hline
\end{tabular}

6.13 Radioluminescence spectra for $\mathrm{NaMgF}_{3}$ nanoparticles doped with $1 \% \mathrm{Eu}$ \begin{tabular}{|c|}
\hline (a) and $2 \% \mathrm{Eu}$ (c) for different doses and the integrated RL spectra as a \\
\hline
\end{tabular} function of dose during continuous X-ray irradiation for $1 \% \mathrm{Eu}$ (b) and 2

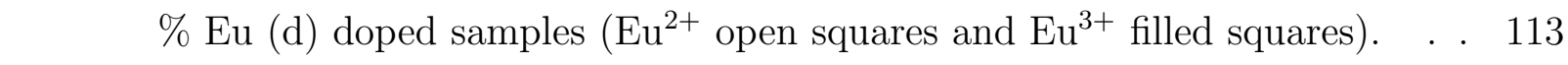


6.14 Radioluminescence spectra for $\mathrm{NaMgF}_{3}$ nanoparticles doped with $5 \% \mathrm{Eu}$

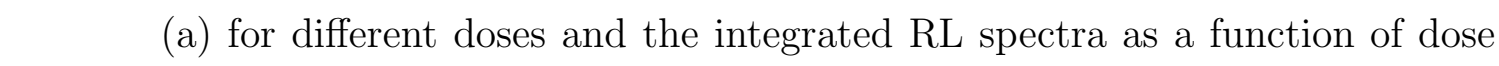
during continuous X-ray irradiation for $5 \% \mathrm{Eu}(\mathrm{b})$ doped samples $\left(\mathrm{Eu}^{2+}\right.$ \begin{tabular}{|c|c|}
\hline open squares and $\mathrm{Eu}^{3+}$ filled squares, solid line ${ }^{241} \mathrm{Am}$ irradiation). The \\
\hline
\end{tabular} $\gamma$-ray RL was scaled to match the X-ray RL. . . . . . . . . . . . . . . . . 114

6.15 The integrated $\mathrm{Eu}^{3+}$ (filled squares, left axis) and $\mathrm{Eu}^{2+}$ (open squares, right axis) RL spectra as a function of radiation dose for $\mathrm{NaMgF}_{3}: 1 \%$ Eu nanoparticles with a crystal size of $57 \mathrm{~nm}[10] \ldots \ldots \ldots$. . . . . . . 116

6.16 Radioluminescence spectra for $\mathrm{NaMgF}_{3}$ nanoparticles doped with $1 \% \mathrm{Mn}$ (a) for different doses and the integrated RL spectra as a function of dose

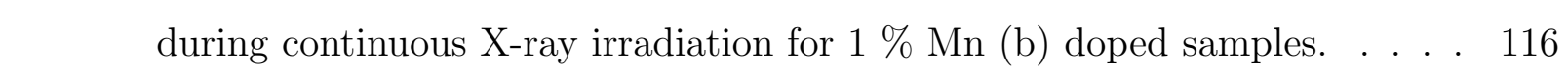

6.17 Radioluminescence spectra for $\mathrm{NaMgF}_{3}$ nanoparticles doped with $2 \% \mathrm{Mn}$ (a) and and $5 \%$ Mn (c), for different doses and the integrated RL spectra \begin{tabular}{|c|}
\hline as a function of dose during continuous X-ray irradiation for $2 \% \mathrm{Mn}(\mathrm{b})$, \\
\hline
\end{tabular} and $5 \% \mathrm{Mn}(\mathrm{d})$ doped samples, solid line ${ }^{241} \mathrm{Am}$ irradiation. The $\gamma$-ray RL

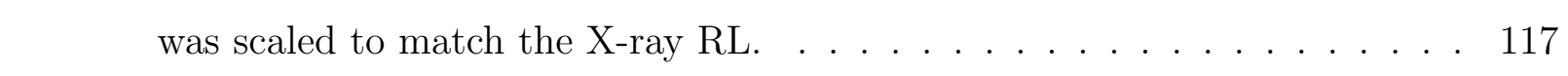

6.18 The integrated RL spectra as a function of radiation dose for $\mathrm{NaMgF}_{3}: 1 \%$

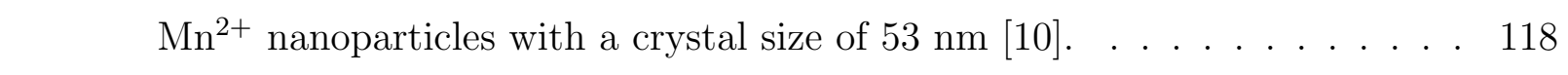

6.19 Temperature dependent RL spectra for $\mathrm{NaMgF}_{3}$ nanoparticles doped with \begin{tabular}{|c|}
\hline $0.1 \% \mathrm{Eu}(\mathrm{a})$, and $5 \% \mathrm{Eu}(\mathrm{c})$ for different temperatures up to $100{ }^{\circ} \mathrm{C}$ and \\
\hline the respective RL spectra versus the wavelength for different temperatures \\
\hline
\end{tabular} ((b) for $0.1 \% \mathrm{Eu}$ and (d) for $5 \% \mathrm{Eu})$. Arrows facing up signal an increase \begin{tabular}{|c|}
\hline of temperature, and arrows facing down signal a decrease in temperature. \\
\hline
\end{tabular} $\mathrm{Eu}^{2+}$ is indicated by the red colour and $\mathrm{Eu}^{3+}$ by the blue colour. The lines are guided to the eye. . . . . . . . . . . . . . . . . 120 6.20 Access of non-radiative traps with increasing temperature. . . . . . . . . . 121 
6.21 Temperature dependent RL spectra for $\mathrm{NaMgF}_{3}$ nanoparticles doped with $1 \% \mathrm{Mn}$ (a) for different temperatures up to $100{ }^{\circ} \mathrm{C}$ and the respective RL spectra versus the wavelength for different temperatures ((b) for $1 \% \mathrm{Mn}$ ). Arrows facing up signal an increase of temperature, and arrows facing down signal a decrease in temperature. The lines are guided to the eye. . . . . . 122

6.22 Temperature dependent RL spectra for $\mathrm{NaMgF}_{3}$ nanoparticles doped with5 $\% \mathrm{Mn}$ (a) for different temperatures up to $100{ }^{\circ} \mathrm{C}$ and the respective RL spectra versus the wavelength for different temperatures ((b) for $5 \% \mathrm{Mn}$ ).

Arrows facing up signal an increase of temperature, and arrows facing down signal an decreases in temperature. The lines are guided to the eye. (c) Non-radiative recombination near a Mn site. . . . . . . . . . . . . . . . . . 124

7.1 (a) PL excitation (dashed curve, $\lambda_{e m}=360$ (blue) $\mathrm{nm}$ and $420 \mathrm{~nm}$ (black)) and emission (solid curve, $\lambda_{e x}=256 \mathrm{~nm}$ ) spectra for $\mathrm{RbMgF}_{3} 0.2 \% \mathrm{Eu}$. (b)

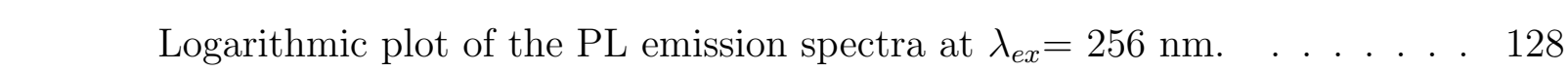

7.2 (a) Absolute PL intensity of the emission spectra, $\lambda_{e x}=256 \mathrm{~nm}$, for $\mathrm{RbMgF}_{3}$ \begin{tabular}{|c|c|}
\hline $0.2 \%$ Eu before (solid curve) and after irradiation with a dose of $12.7 \mathrm{kGy}$ \\
\hline
\end{tabular} (dashed curve). (b) PL excitation at $\lambda_{e m}=590 \mathrm{~nm}$ (blue: solid curve - before irradiation, dashed curve - after irradiation) and emission at $\lambda_{e x}=396 \mathrm{~nm}$ (red: solid curve - before irradiation, dashed curve - after irradiation with a \begin{tabular}{|c|}
\hline dose of $12.7 \mathrm{kGy}$ ) spectra for the $\mathrm{Eu}^{3+}$ ion. (c) Absolute PL intensity of the \\
\hline
\end{tabular} excitation spectra, $\lambda_{e m}=590 \mathrm{~nm}$, before (solid curve) and after irradiation \begin{tabular}{|c|}
\hline with a dose of 12.7 kGy (dashed curve). (d) Absolute PL intensity of the \\
\hline
\end{tabular} emission spectra, $\lambda_{e x}=396 \mathrm{~nm}$, before (solid curve) and after irradiation

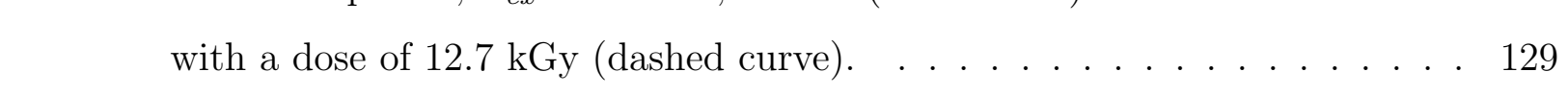

7.3 (a) PL lifetime decay of $0.2 \%$ Eu doped $\mathrm{RbMgF}_{3}$ at $\lambda_{e m}=360 \mathrm{~nm}$ (solid curve) and (b) $\lambda_{e m}=400 \mathrm{~nm}$ emission (solid curve). The dashed curve is the exponential fit. Both lifetimes were measured at $\lambda_{e x}=256 \mathrm{~nm} . \quad$. . . . 131 
7.4 (a) PL excitation spectrum at $\lambda_{e m}=550 \mathrm{~nm}$ (dashed curve) and $\lambda_{e m}=580$ nm emission (solid curve). (b) PL emission spectrum for $\mathrm{RbMgF}_{3}$ doped

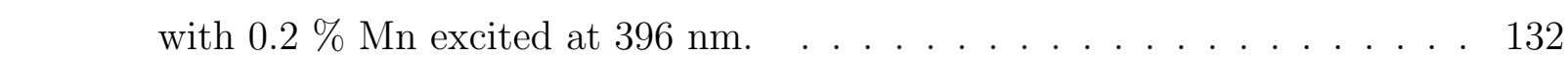

7.5 (a) PL lifetime decay of $0.2 \% \mathrm{Mn}$ doped $\mathrm{RbMgF}_{3}$ at $\lambda_{e m}=550 \mathrm{~nm}$ (solid \begin{tabular}{|c|c|}
\hline curve) and (b) $\lambda_{e m}=580 \mathrm{~nm}$ emission (solid curve). The dashed curve is \\
\hline
\end{tabular} \begin{tabular}{|c|c|c|}
\hline the exponential fit. Both lifetimes were measured at $\lambda_{e x}=465 \mathrm{~nm}$. \\
\hline
\end{tabular}

7.6 (a) TL for the $\mathrm{RbMgF}_{3}$ sample doped with $0.2 \% \mathrm{Eu}$ (solid curve). The TL \begin{tabular}{|c|}
\hline data was detected with the PMT after 5 min X-ray irradiation $(40 \mathrm{kV}, 40$ \\
\hline
\end{tabular} $\mathrm{mA}$ ) at a heating rate of $1 \mathrm{~K} / \mathrm{s}$. The fitted peaks were obtained using first order kinetics glow curve deconvolution (dashed curves). (b) TL spectra vs.

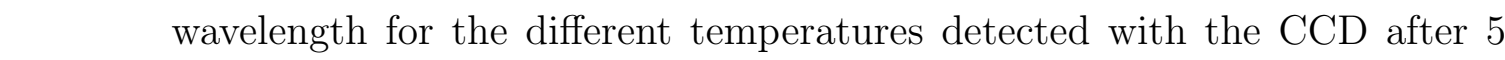
min X-ray irradiation. (c) TL for the unknown peak between $500 \mathrm{~nm}$ and $650 \mathrm{~nm}$ (solid curve). TL was detected with the CCD after 5 min of X-ray irradiation and the peaks were fitted using first order kinetics glow curve deconvolution (dashed curves). . . . . . . . . . . . . 136

7.7 (a) TL for the $\mathrm{RbMgF}_{3}$ sample doped with $0.2 \% \mathrm{Mn}$ (solid curve). The TL signal was detected with the PMT after 1 min X-ray irradiation $(40 \mathrm{kV}, 40$ $\mathrm{mA}$ ) at a heating rate of $1 \mathrm{~K} / \mathrm{s}$. The fitted peaks were obtained using first order kinetics glow curve deconvolution (dashed curves). (b) TL spectra vs. wavelength for the different temperatures detected with the CCD after 5 min X-ray irradiation. (c) Normalised TL spectra at 341.5 K, $477 \mathrm{~K}$ and 622 K. . . . . . . . . . . . . . . . . . . . . . . . 138

7.8 (a) Radioluminescence spectra for $\mathrm{RbMgF}_{3}: 0.2 \% \mathrm{Eu}$ versus the dose and (b) the integrated RL spectra as a function of dose during continuous Xray irradiation $\left(\mathrm{Eu}^{2+}\right.$ : filled squares, $\mathrm{Eu}^{3+}$ : open squares, unknown defect: circles). (c) Existence of electron and hole traps next to the Eu. (d) Dose dependence of the RL signal as a result of the generation of non-radiative recombination centres. . . . . . . . . . . . . . . . . . . . . . 141 
7.9 (a) PL, TL and RL emission spectra for the broad peak around $400 \mathrm{~nm}$ to $580 \mathrm{~nm}$. (b) Different excitation regions for PL and RL. . . . . . . . . . . . 142

7.10 (a) Radioluminescence spectra for $\mathrm{RbMgF}_{3}: 0.2 \%$ Mn for versus the dose \begin{tabular}{|c|}
\hline and (b) the integrated RL spectra as a function of dose during continuous \\
\hline
\end{tabular} X-ray irradiation. . . . . . . . . . . . . . . . . . 143

7.11 (a) Temperature dependent RL for $\mathrm{RbMgF}_{3}: 0.2 \% \mathrm{Eu}$ for different temperature up to $100{ }^{\circ} \mathrm{C}$. Arrows facing up signal an increase of temperature, and arrows facing down signal a decrease in temperature. (b) RL spectra measured at different temperatures. (c) Hopping of the carriers to a deep non-radiative trap due to increasing temperature. . . . . . . . . . . . . . . 145

7.12 (a) Temperature dependent RL for $\mathrm{RbMgF}_{3}: 0.2 \%$ Mn for different temperature up to $100{ }^{\circ} \mathrm{C}$. Arrows facing up signal an increase of temperature, \begin{tabular}{|c|}
\hline and arrows facing down signal a decrease in temperature. (b) RL spectra \\
\hline
\end{tabular} measured for different temperatures. . . . . . . . . . . . . . . 147

8.1 (a) PL excitation for the $\mathrm{Eu}^{2+}\left(\lambda_{e m}=360 \mathrm{~nm}\right.$ - dashed curve $)$ and $\mathrm{Eu}^{3+}$ $\left(\lambda_{e m}=592 \mathrm{~nm}\right.$ - solid curve) and (b) emission $\left(\lambda_{e x}=396 \mathrm{~nm} ;\right.$ inset $\lambda_{e x}=256$ $\mathrm{nm})$ spectra for $\mathrm{RbMgF}_{3}$ doped with $1 \% \mathrm{Eu}$ (dashed curve) and $5 \% \mathrm{Eu}$ (solid curve). . . . . . . . . . . . . . . . . . . 150

8.2 (a) PL emission spectra for $0.1 \%$, (b) $0.5 \%$, (c) $1 \%$ and (d) $5 \%$ Eu doped $\mathrm{RbMgF}_{3}$ at $\lambda_{e x}=396 \mathrm{~nm}$ at $10 \mathrm{~K}$ (solid curve) and at room temperature (dashed curve). Inset: PL excitation spectra detected at $\lambda_{e m}=450 \mathrm{~nm}$ for the different Eu concentrations of $\mathrm{RbMgF}_{3} . \ldots \ldots$. . . . . . . . . 152 
8.3 (a) Temperature dependent average PL lifetime of $1 \%$ Eu doped $\mathrm{RbMgF}_{3}$ \begin{tabular}{|c|}
\hline$\left(\lambda_{e m}=592 \mathrm{~nm} ; \lambda_{e x}=375 \mathrm{~nm}\right)$ from the three exponential fit of the data \\
\hline with only the $\tau_{1}$ (filled squares), $\tau_{2}$ (filled circles), $\tau_{3}$ (filled triangles) and the \\
\hline average lifetime, $\tau_{123, \text { ave }}$ (open squares). $\tau_{3}$ was fitted using equation 8.1 . (b) \\
\hline
\end{tabular} Temperature dependent average PL lifetime of $1 \%$ Eu doped $\mathrm{RbMgF}_{3}$ from the three exponential fit of the data with only the $\tau_{1}$ (filled squares), $\tau_{2}$ (filled circles) and the average lifetime, $\tau_{12, a v e}$ (open squares) when only using the first and the second component. . . . . . . . . . . . . . . . . 154

8.4 (a) Temperature dependent average PL lifetime of $5 \%$ Eu doped $\mathrm{RbMgF}_{3}$ \begin{tabular}{|c|}
\hline$\left(\lambda_{e m}=592 \mathrm{~nm} ; \lambda_{e x}=375 \mathrm{~nm}\right)$ from the three exponential fit of the data \\
\hline with only the $\tau_{1}$ (filled squares), $\tau_{2}$ (filled circles), $\tau_{3}$ (filled triangles) and the \\
\hline average lifetime, $\tau_{123, \text { ave }}$ (open squares). $\tau_{3}$ was fitted using equation 8.1 . ( $\left.\mathrm{b}\right)$ \\
\hline Temperature dependent average PL lifetime of $5 \%$ Eu doped $\mathrm{RbMgF}_{3}$ from \\
\hline the three exponential fit of the data with only the $\tau_{1}$ (filled squares), $\tau_{2}$ (filled \\
\hline circles) and the average lifetime, $\tau_{12, \text { ave }}$ (open squares) when only using the \\
\hline
\end{tabular} first and the second component. . . . . . . . . . . . . . . . 155

8.5 (a) Average PL lifetime for $\mathrm{Eu}^{3+}\left(\lambda_{e m}=592 \mathrm{~nm}, \lambda_{e x}=375 \mathrm{~nm}\right)$ doped $\mathrm{RbMgF}_{3}$ nanoparticles against the rare earth concentration and (b) for the broad unknown peak $\lambda_{e m}=500 \mathrm{~nm}$ and $\lambda_{e x}=375 \mathrm{~nm}$. The measurements were done at room temperature. . . . . . . . . . . . . . 156

$8.6 \quad$ (a) Average PL lifetime for $\mathrm{Eu}^{2+}\left(\lambda_{e m}=360 \mathrm{~nm}\right.$ : open squares and $\lambda_{e m}=380$ \begin{tabular}{|c|}
\hline $\mathrm{nm}$ : filled squares at $\lambda_{e x}=256 \mathrm{~nm}$ ) doped $\mathrm{RbMgF}_{3}$ nanoparticles against \\
\hline the rare earth concentration and (b) average PL lifetime for $\mathrm{Eu}^{2+}\left(\lambda_{e m}=360\right.$ \\
\hline $\mathrm{nm}$ : open squares and $\lambda_{e m}=380 \mathrm{~nm}$ : filled squares at $\lambda_{e x}=256 \mathrm{~nm}$ ) doped \\
\hline $\mathrm{NaMgF}_{3}$ nanoparticles against the rare earth concentration. (c) PL lifetime \\
\hline for the $\mathrm{Eu}^{3+}$ ion with $\lambda_{e m}=590 \mathrm{~nm}$ and $\lambda_{e x}=375 \mathrm{~nm}$ for the $\mathrm{RbMgF}_{3}$ \\
\hline (circles) and $\mathrm{NaMgF}_{3}$ (squares). (d) PL lifetime for the broad peak at $\lambda_{e m}=$ \\
\hline $500 \mathrm{~nm}$ and $\lambda_{e x}=375 \mathrm{~nm}$ for the $\mathrm{RbMgF}_{3}$ (circles) and $\mathrm{NaMgF}_{3}$ (squares). \\
\hline
\end{tabular}

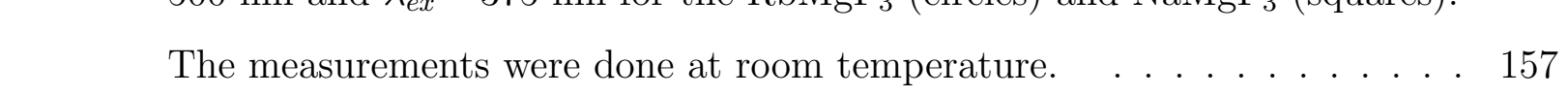


8.7 (a) PL lifetime decay of $5 \%$ Eu doped $\mathrm{RbMgF}_{3}$ at $\lambda_{e m}=590 \mathrm{~nm}$ (solid curve). The dashed curve is the exponential fit. The lifetime was measured at $\lambda_{e x}=375 \mathrm{~nm}$. (b) The lifetime decay curves at $\lambda_{e m}=590 \mathrm{~nm}$ emission for the different Eu concentrations in $\mathrm{NaMgF}_{3} . \quad$. . . . . . . . . . . . . . 160

8.8 Quantum efficiency, $\eta$, for $\mathrm{RbMgF}_{3}$ and $\mathrm{NaMgF}_{3}$ against the Eu concentration. 162

8.9 The Judd-Ofelt parameters $\Omega_{2}$ and $\Omega_{4}$ for the $\mathrm{Eu}^{3+}$ ions for $\mathrm{RbMgF}_{3}$ (a) and $\mathrm{NaMgF}_{3}$ (b) against the Eu concentration. . . . . . . . . . . . . 163

8.10 (a) PL excitation spectra for $5 \% \mathrm{Mn}$ doped $\mathrm{RbMgF}_{3}$ at $\lambda_{e m}=500 \mathrm{~nm}$ (dashed curve) and $\lambda_{e m}=600 \mathrm{~nm}$ (solid curve) for the distorted $\mathrm{Mn}^{2+}$ site \begin{tabular}{|c|c|}
\hline [11]. (b) PL emission spectrum for $\mathrm{RbMgF}_{3}$ doped with $1 \% \mathrm{Mn}$ (red), $2 \%$ \\
\hline
\end{tabular} (blue) and $5 \% \mathrm{Mn}$ (black) excited at $396 \mathrm{~nm}[11] . \quad$. . . . . . . . . . . 164

8.11 (a) PL lifetime decay of $5 \% \mathrm{Mn}$ doped $\mathrm{RbMgF}_{3}$ at $\lambda_{e m}=550 \mathrm{~nm}$ (solid \begin{tabular}{|c|c|}
\hline curve) and (b) at $\lambda_{e m}=580 \mathrm{~nm}$ (solid curve). The dashed curve is the \\
\hline
\end{tabular} \begin{tabular}{|l}
\hline exponential fit. The lifetime was measured at $\lambda_{e x}=465 \mathrm{~nm}$. \\
\hline
\end{tabular} . . . . . . 165

8.12 Average PL lifetime for the two inequivalent sites of $\mathrm{Mn}^{2+}\left(\lambda_{e m}=550 \mathrm{~nm}\right.$ - circles and $\lambda_{e m}=580 \mathrm{~nm}$ - squares) in $\mathrm{RbMgF}_{3}$ nanoparticles against the rare earth concentration $\left(\lambda_{e x}=465 \mathrm{~nm}\right) . \ldots \ldots \ldots \ldots$

8.13 TL glow curves for $\mathrm{RbMgF}_{3}$ doped with $1 \%$ (a), $2 \%$ (b) and $5 \%$ (c) Eu after 20 min irradiation with the X-ray generator operating at $40 \mathrm{kV}$ and $40 \mathrm{~mA}$ \begin{tabular}{|c|}
\hline (solid curves). The TL was detected with the PMT. The fitted peaks were \\
\hline obtained using first order kinetics glow curve deconvolution (dashed curves).
\end{tabular} (d) Normalised TL glow curves for $1 \%$ Eu doped $\mathrm{RbMgF}_{3}$ nanoparticles (blue curve), $0.5 \%$ Eu doped $\mathrm{NaMgF}_{3}$ nanoparticles (green curve) and 0.2

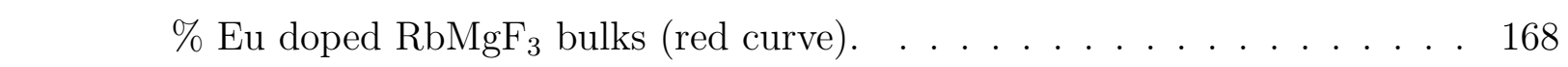


8.14 TL glow curves for $\mathrm{RbMgF}_{3}$ doped with $1 \% \mathrm{Mn}(\mathrm{a}), 2 \% \mathrm{Mn}(\mathrm{b}), 5 \%$ Mn (c) after 20 min irradiation with the X-ray generator operating at 40 $\mathrm{kV}$ and $40 \mathrm{~mA}$ (solid curves). The TL was detected with the PMT. The fitted peaks were obtained using first order kinetics glow curve deconvolution (dashed curves). (d) Normalised TL glow curves for $5 \%$ Mn doped $\mathrm{RbMgF}_{3}$ nanoparticles (blue curve), $5 \% \mathrm{Mn}$ doped $\mathrm{NaMgF}_{3}$ nanoparticles (green

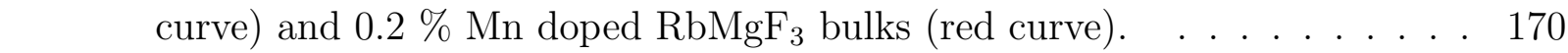

8.15 Radioluminescence spectra for $\mathrm{RbMgF}_{3}$ nanoparticles doped with $0.1 \% \mathrm{Eu}$ (a) and $0.5 \% \mathrm{Eu}$ (c) for different doses and the integrated RL spectra as a

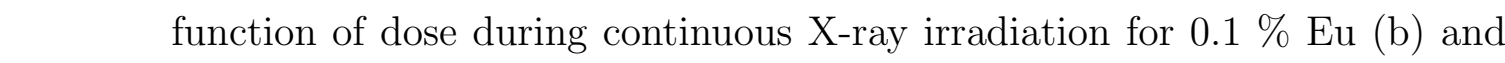

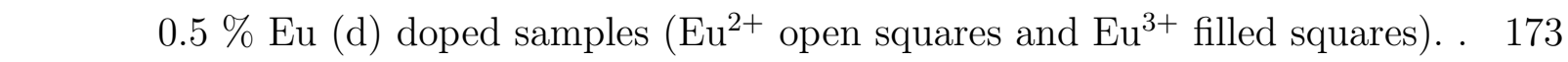

8.16 Radioluminescence spectra for $\mathrm{RbMgF}_{3}$ nanoparticles doped with $1 \% \mathrm{Eu}$ (a) and $2 \% \mathrm{Eu}$ (c) for different doses and the integrated RL spectra as a function of dose during continuous X-ray irradiation for $1 \% \mathrm{Eu}$ (c) and 2

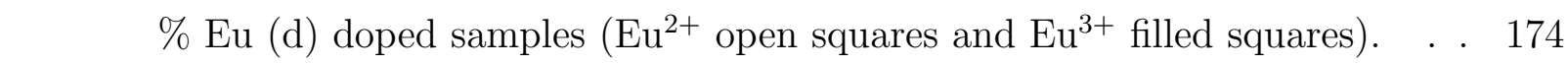

8.17 Radioluminescence spectra for $\mathrm{RbMgF}_{3}$ nanoparticles doped with $5 \% \mathrm{Eu}$ \begin{tabular}{|c|c|c|c|}
\hline (a) for different doses and the integrated RL spectra as a function of dose \\
\hline
\end{tabular} during continuous X-ray irradiation for $5 \% \mathrm{Eu}(\mathrm{b})$ doped samples $\left(\mathrm{Eu}^{2+}\right.$ open squares and $\mathrm{Eu}^{3+}$ filled squares, solid line ${ }^{241} \mathrm{Am}$ irradiation). The $\gamma$-ray RL was scaled to match the X-ray RL. . . . . . . . . . . . . . . . . . 175

8.18 Radioluminescence spectra for $\mathrm{RbMgF}_{3}$ nanoparticles doped with $1 \% \mathrm{Mn}$

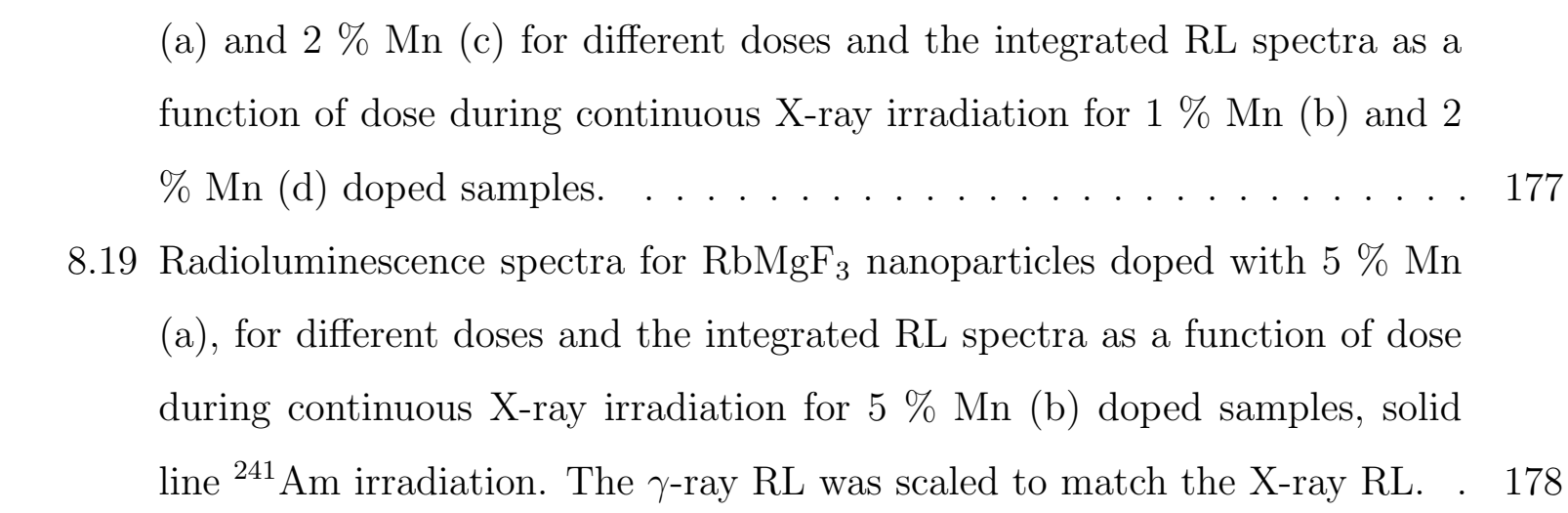


8.20 Temperature dependent RL spectra for $\mathrm{RbMgF}_{3}$ nanoparticles doped with $0.1 \% \mathrm{Eu}$ (a) for different temperatures up to $100{ }^{\circ} \mathrm{C}$ and the respective RL spectra versus the wavelength for different temperatures ((b) for $0.1 \% \mathrm{Eu})$. $\mathrm{Eu}^{2+}$ is indicated by the red colour and $\mathrm{Eu}^{3+}$ by the blue colour. Arrows facing up signal an increase of temperature, and arrows facing down signal a decrease in temperature. Black curves show the trend of the temperature dependent RL.

8.21 Temperature dependent RL spectra for $\mathrm{RbMgF}_{3}$ nanoparticles doped with

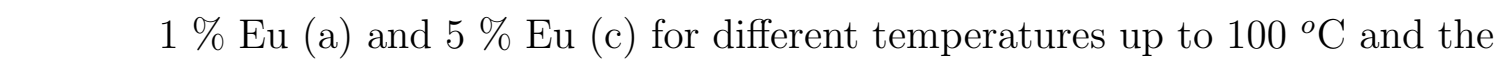
respective RL spectra versus the wavelength for different temperatures ((b) for $1 \% \mathrm{Eu}$ and (d) for $5 \% \mathrm{Eu}) . \mathrm{Eu}^{2+}$ is indicated by the red colour and $\mathrm{Eu}^{3+}$ by the blue colour. Arrows facing up signal an increase of temperature, and arrows facing down signal a decrease in temperature. Black curves show the trend of the temperature dependent RL. . . . . . . . . . . . . . . . . . 182

8.22 Temperature dependent RL spectra for $\mathrm{RbMgF}_{3}$ nanoparticles doped with \begin{tabular}{|c|}
\hline $1 \% \mathrm{Mn}(\mathrm{a})$ and $5 \% \mathrm{Mn}$ (c) for different temperatures up to $100{ }^{\circ} \mathrm{C}$ and \\
\hline the respective RL spectra versus the wavelength for different temperatures \\
\hline ((b) for $1 \% \mathrm{Mn}$ and (d) for $5 \% \mathrm{Mn})$. Arrows facing up signal an increase \\
\hline
\end{tabular} of temperature, and arrows facing down signal a decrease in temperature.

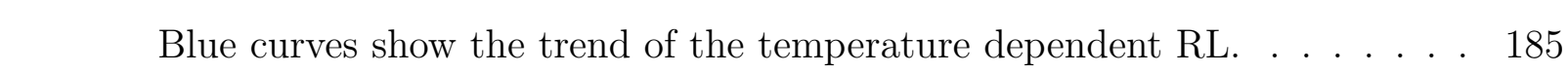

9.1 (a) PL excitation $\left(\lambda_{e m}=590 \mathrm{~nm}\right)$ and (b) PL emission $\left(\lambda_{e x}=395 \mathrm{~nm}\right)$ spectra for $\mathrm{LaF}_{3}: 5 \% \mathrm{Eu}^{3+}$ nanoparticles. (c) PL emission $\left(\lambda_{e x}=395 \mathrm{~nm}\right)$ spectra for all the different $\mathrm{Eu}^{3+}$ concentrations in $\mathrm{LaF}_{3}$ nanoparticles [12]. . . . . 191

9.2 (a) Average PL lifetime for $\mathrm{Eu}^{3+}\left(\lambda_{e m}=590 \mathrm{~nm}, \lambda_{e x}=400 \mathrm{~nm}\right)$ doped $\mathrm{LaF}_{3}$ samples against the rare earth concentration. The line is guided to the eye [12]. (b) PL lifetime decay of $5 \%$ Eu doped $\mathrm{LaF}_{3}$ at $\lambda_{e m}=590 \mathrm{~nm}$ (black curve). The red curve is the exponential fit. The lifetime was measured at $\lambda_{e m}=400 \mathrm{~nm}$. . . . . . . . . . . . . . . . . . . . . 193 
9.3 (a) PL excitation $\left(\lambda_{e m}=600 \mathrm{~nm}\right)$ and (b) PL emission $\left(\lambda_{e x}=400 \mathrm{~nm}\right)$ spectra for $\mathrm{LaF}_{3}: 5 \% \mathrm{Sm}^{3+}$ nanoparticles [12]. . . . . . . . . . . . . . . . . . 195

9.4 Cross relaxation process between $\mathrm{Sm}^{3+}$ ion [13]. . . . . . . . . . . . . 196

9.5 (a) Average PL lifetimes for $\mathrm{Sm}^{3+}\left(\lambda_{e m}=600 \mathrm{~nm}, \lambda_{e x}=400 \mathrm{~nm}\right)$ doped $\mathrm{LaF}_{3}$ samples against the rare earth concentration. The line is guided to the eye [12]. (b) PL lifetime decay of $5 \% \mathrm{Sm}$ doped $\mathrm{LaF}_{3}$ at $\lambda_{\text {em }}=600 \mathrm{~nm}$ (black

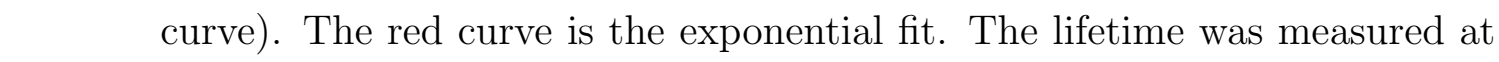
$\lambda_{e m}=400 \mathrm{~nm}$. . . . . . . . . . . . . . . . . 197

9.6 (a) Radioluminescence spectra for $\mathrm{LaF}_{3}: 5 \% \mathrm{Eu}^{3+}$ nanoparticles during continuous X-ray irradiation. (b) The integrated RL spectra as a function of irradiation time for $\mathrm{LaF}_{3}: 5 \% \mathrm{Eu}^{3+}$ nanoparticles during continuous Xray irradiation (filled squares) or $60 \mathrm{keV} \gamma$-ray irradiation (solid line). The

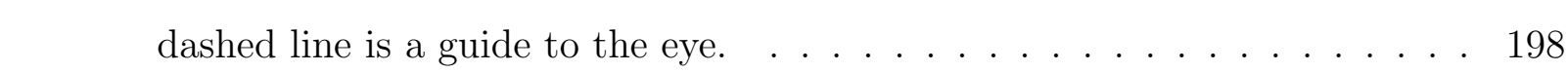

9.7 (a) Radioluminescence spectra for (a) $\mathrm{LaF}_{3}: 5 \% \mathrm{Sm}^{3+}$ nanoparticles. (b) \begin{tabular}{|c|}
\hline The integrated RL spectra as a function of irradiation time for $\mathrm{LaF}_{3}: 5$ \\
\hline$\% \mathrm{Sm}^{3+}$
\end{tabular} $\% \mathrm{Sm}^{3+}$ nanoparticles. The radioluminescence data were recorded during continuous X-ray irradiation. . . . . . . . . . . . . . . . . 200

9.8 (a) PL excitation $\left(\lambda_{e m}=590 \mathrm{~nm}\right)$ and (b) PL emission $\left(\lambda_{e x}=395 \mathrm{~nm}\right)$ spectra for $\mathrm{BaF}_{2}: 5 \% \mathrm{Eu}^{3+}$ nanoparticles. . . . . . . . . . . . . . . . 201

9.9 (a) Radioluminescence spectra for $\mathrm{BaF}_{2}: 5 \% \mathrm{Eu}^{3+}$ nanoparticles during continuous X-ray irradiation after 68 Gy (black line) and 6.5 kGy (blue line). (b) The integrated RL spectra as a function of irradiation time for $\mathrm{BaF}_{2}: 5 \% \mathrm{Eu}^{3+}$ nanoparticles during continuous X-ray irradiation 202 9.10 (a) PL excitation $\left(\lambda_{e m}=630 \mathrm{~nm}\right)$ and (b) PL emission $\left(\lambda_{e x}=396 \mathrm{~nm}\right)$ spectra for $\mathrm{BaMgF}_{4}: 5 \% \mathrm{Mn}^{2+}$ nanoparticles. . . . . . . . . . . . . . 203

9.11 (a) Radioluminescence spectra for $\mathrm{BaMgF}_{4}: 5 \% \mathrm{Mn}^{2+}$ nanoparticles during \begin{tabular}{|l|}
\hline continuous X-ray irradiation after 68 Gy (black line) and $6.5 \mathrm{kGy}$ (blue line). \\
\hline (b) The integrated RL spectra as a function of irradiation time for $\mathrm{BaMgF}_{4}$ : \\
\hline $5 \% \mathrm{Mn}^{2+}$ nanoparticles during continuous X-ray irradiation. . . . . . . . 204
\end{tabular} 


\section{List of Tables}

$2.1 \quad$ Typical radiation doses applied in dosimetry; Gy is the unit Gray with 1

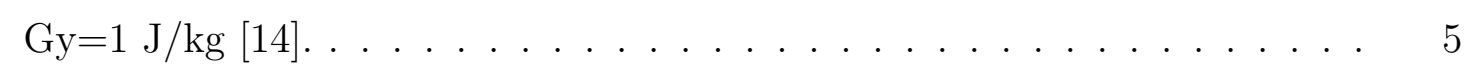

2.2 Summary of radiation sickness after various doses of radiation (Sievert $=\mathrm{Sv}=\mathrm{J} / \mathrm{kg}$ )

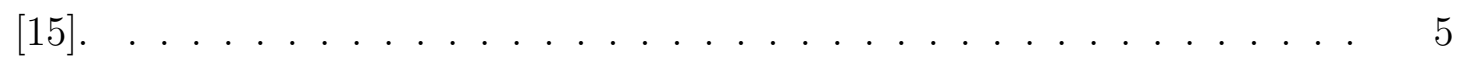

2.3 Typically effective doses applied during medical examination [15, 16, 17]. . 21

2.4 Types of cancer treatment with typically applied doses (in Gy) [15, 16, 17]. 23

$3.1 \quad$ Selection rules for the forced electric dipole process, magnetic dipole process

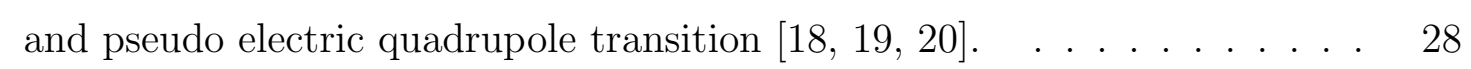

$5.1 \quad$ Different compounds with the pre factors of the PL lifetimes of $0.2 \% \mathrm{Eu}$ doped $\mathrm{NaMgF}_{3} . \ldots \ldots \ldots \ldots \ldots$

$5.2 \quad$ Average PL lifetimes of $0.2 \%$ Eu doped $\mathrm{NaMgF}_{3}$. . . . . . . . . . . . 59

5.3 Different compounds with the pre factors of the PL lifetimes of Mn doped

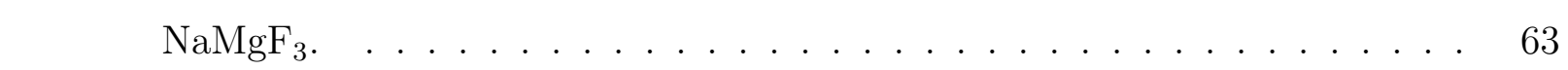

5.4 Average PL lifetimes of distorted and regular $\mathrm{Mn}^{2+}$ sites in $\mathrm{NaMgF}_{3}$. . . . 64

5.5 The activation energies of the individual glow curve peaks for the $\mathrm{NaMgF}_{3}: 0.2$ $\%$ Eu sample obtained by first order kinetics glow curve deconvolution. The top line is the peak temperature for the glow curve. $\ldots \ldots$. . . . . 66 
5.6 The activation energies of the individual glow curve peaks for $\mathrm{NaMgF}_{3}$ doped with different concentrations of Mn obtained by first order kinetics glow curve deconvolution. The top line is the activation energy for the different peaks for the glow curves. . . . . . . . . . . . . . . . . . . . 72

5.7 Summary of temperature changes of the RL signal for different doped samples and different $\mathrm{Mn}^{2+}$ sites. . . . . . . . . . . . . . . . . . . 85

6.1 Summary of the different measured PL lifetimes, $\tau_{12, \text { ave }}$, the radiative lifetime, $\tau_{\text {rad }}$, and the quantum efficiency, $\eta$, for $\mathrm{NaMgF}_{3}$ doped with different concentrations of Eu. . . . . . . . . . . . . . . . . . . . . . . 97

6.2 The Judd-Ofelt parameters $\Omega_{2}$ and $\Omega_{4}$ for ${ }^{5} \mathrm{D}_{0} \rightarrow{ }^{7} \mathrm{~F}_{2}$ transitions for different concentrations of Eu doped $\mathrm{NaMgF}_{3}$ nanoparticles measured using the PL

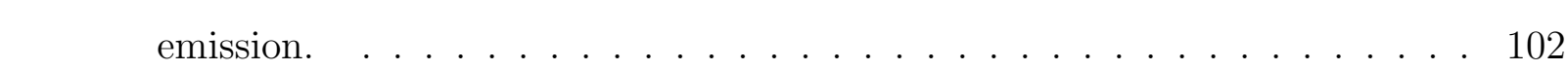

6.3 Summary of different measured PL lifetimes, $\tau_{\text {ave }}$, for $\mathrm{NaMgF}_{3}$ doped with

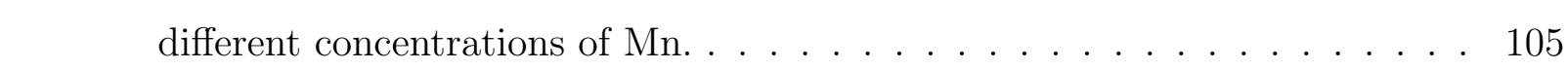

6.4 The activation energies of the individual glow curve peaks for the $\mathrm{NaMgF}_{3}$ :

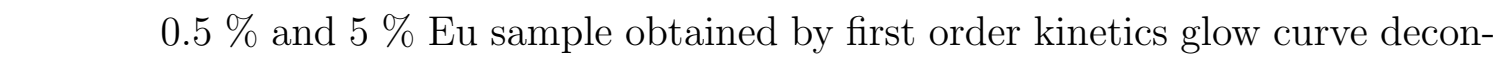
volution. The top line is the activation energy for the glow curve. . . . . . 107

6.5 The activation energies of the individual glow curve peaks for $\mathrm{NaMgF}_{3}$ doped with different concentration of $\mathrm{Mn}^{2+}$ obtained by first order kinetics glow curve deconvolution. The top line is the activation energy for the different peaks for the glow curves. . . . . . . . . . . . . . . . . . . . . . 110

7.1 Different compounds with the pre factors of the PL lifetimes of Eu doped

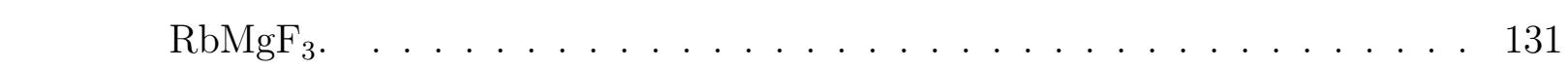

7.2 Different compounds with the pre factors of the PL lifetimes of Mn doped $\mathrm{RbMgF}_{3} \ldots \ldots \ldots \ldots \ldots \ldots \ldots \ldots \ldots \ldots \ldots \ldots$

7.3 The activation energies of the individual glow curve peaks for the $\mathrm{RbMgF}_{3}: 0.2$ \% Eu sample obtained by first order kinetics glow curve deconvolution. The top line is the activation energy of the peaks for the for the glow curve. 137 
7.4 The activation energies of individual glow curve peaks for the $\mathrm{RbMgF}_{3}: 0.2$ $\%$ Mn sample obtained by first order kinetics glow curve deconvolution. The top line is the peak temperature for the glow curve. $\ldots$. . . . . . . . . 139

8.1 Summary of the different measured PL lifetimes, $\tau_{12, \text { ave }}$, the radiative lifetime, $\tau_{\text {rad }}$, and the quantum efficiency, $\eta$, for $\mathrm{RbMgF}_{3}$ doped with different

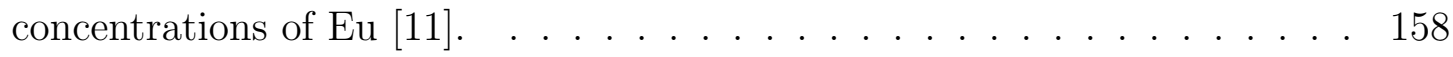

8.2 Summary of the measured PL lifetimes, $\tau_{\text {ave }}$, for different Eu-ions and the broad peak around $470 \mathrm{~nm}$ for $\mathrm{RbMgF}_{3}$ and $\mathrm{NaMgF}_{3}$ bulk materials. . . . 161

8.3 The Judd-Ofelt parameters $\Omega_{2}$ and $\Omega_{4}$ for ${ }^{5} \mathrm{D}_{0} \rightarrow{ }^{7} \mathrm{~F}_{2}$ transitions for different concentrations of Eu doped $\mathrm{RbMgF}_{3}$ nanoparticles measured using the PL emission. . . . . . . . . . . . . . . . . . . 163

8.4 Summary of different measured PL lifetimes, $\tau_{\text {ave }}$, for $\mathrm{RbMgF}_{3}$ doped with different concentrations of $\mathrm{Mn}$, exited at $465 \mathrm{~nm}$. . . . . . . . . . . . 167

8.5 The activation energies of the individual glow curve peaks for the $\mathrm{RbMgF}_{3}: 1$

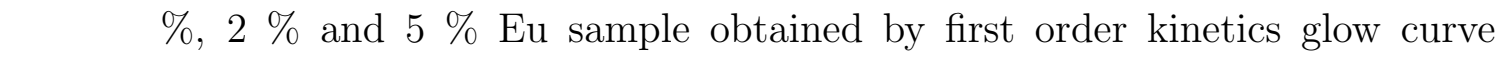
deconvolution. The top line is the activation energy for the glow curve. . . 169

8.6 The activation energies of the individual glow curve peaks for $\mathrm{RbMgF}_{3}$ doped with different concentration of Mn obtained by first order kinetics glow curve deconvolution. The top line is the activation energy for the different peaks for the glow curves. . . . . . . . . . . . . . . . . . . . . . 171

8.7 Summary of thermal coefficients of the RL signal for different doped samples and the $\mathrm{Eu}^{2+}$ and $\mathrm{Eu}^{3+}$ ions in $\mathrm{RbMgF}_{3}$ nanoparticles. . . . . . . . . . . . 183

8.8 Summary of the thermal coefficient of the RL signal for the different doped samples and the $\mathrm{Mn}^{2+}$ ion in $\mathrm{RbMgF}_{3}$ nanoparticles. . . . . . . . . . . . . 184

9.1 Summary of different measured PL lifetimes, $\tau_{\text {ave }}$ and the quantum efficiency, $\eta$, for $\mathrm{LaF}_{3}$ doped with different concentrations of $\mathrm{Eu}^{3+}[12]$.

9.2 Summary of the different measured PL lifetimes, $\tau_{\text {ave }}$, for $\mathrm{LaF}_{3}$ doped with different concentrations of $\mathrm{Sm}^{3+}$. . . . . . . . . . . . . . . . . . . 197 
9.3 Summary of the dose dependence of the RL signal for different fluoride compounds after receiving a dose of $\sim 10 \mathrm{kGy} . ~ \ldots . . . . .205$ 


\section{Introduction}

Fluoroperovskite-based compounds have recently attracted interest because of the range of potential applications including optical amplifiers [21, 22] and radiation detectors [23, 24]. Bulk materials and nanoparticles have the potential to be used in small sized and remote dosimeters that can be employed in a number of applications i.e. determining the real-time radiation dose and dose rate in radiation protection, non-destructive testing, and in medical radiotherapy for monitoring the prescribed radiation for dose verification and validation. Transparent nanopartilces/ polymer composites are particularly useful for this application, because they can be used to construct long fibre optic dosimeters that take advantage of the reduced light scattering. Real-time read-out can be performed with radioluminescence $(\mathrm{RL})$. This is a phenomenon that occurs from the prompt radiative recombination of charged particles after irradiation. There is a need for new RL materials that have good transparency, high sensitivity and with a response to radiation that is equivalent to that of tissue. Dose verification and validation can also be done with thermoluminescence (TL) materials, which is a phenomenon where irradiation induces carriers that become trapped. These carriers are released by heating the sample resulting in recombination and light emission. New TL materials with tissue equivalent characteristics will find applications in the area of personal dosimetry.

This work presents an investigation into the use of bulk materials and nanoparticles for real-time dosimetry and the determination of the total dose after irradiation. In chapter 2 , basic theory on the quantities to describe the interaction of radiation with matter and the energy dependence is presented. Chapter 3 explains the theoretical background of 
the techniques, which are employed and the luminescence characteristics of the rare earth ions. Experimental techniques and a description of sample preparations are outlined in chapter 4 . In chapters $5-8$, potential materials for tissue equivalent applications in radiation dosimetry are explored. New measurements were performed to investigate the RL and temperature dependent RL behaviour of bulk materials. The photoluminescence (PL) after heavy irradiation was also investigated. For the fluoroperovskite nanoparticles temperature dependent PL and PL lifetime measurements were performed. Their RL, TL and temperature dependent RL was determined. New defects were observed in both the bulk materials and nanoparticles and a detailed model for the trap distribution was developed. Chapter 9 presents the final results chapter and an overview of other fluoride nanoparticles and their respective RL characteristics. Chapter 10 provides a brief summary and an outlook from this thesis. 


\section{Basic quantities and units in radiation dosimetry}

The motivation of today's research is to find new transparent dosimeters for different energy regions and applications. A good transparency is important for the samples, because during the stimulation of the sample the emitted light will not be scattered in the material. To better understand the quantities utilised in radiation dosimetry and the different energy regions for their applications, a short overview is outlined in this chapter.

To measure the radiation, which is incident on a material or person, is of great interest. For devices that are to be used in space will degrade with the amount of radiation they receive, or in the human context radiation damages the DNA in biological cells. In radiotherapy, it is desirable for thermoluminescence dosimeters (TLD) and optically stimulated luminescence (OSLD) materials to have an effective atomic number $\left(Z_{e f f}\right)$ near that of human tissue $\left(\mathrm{Z}_{e f f}=7.6\right)$. This means the response to radiation is equivalent to tissue. In personal dosimetry, the doses range from $1 \mu \mathrm{Sv}$ to $1 \mathrm{~Sv}$ and the uncertainty of the detector material should be less than $4 \%$ for radiotherapy, otherwise the damage of healthy tissue would be too high [6]. Therefore, there is a need for dosimeters to measure the radiation to know if the dose delivered to the devices/ humans is harmful [14]. In the past, there have been many different types of dosimeters developed, for example:

Geiger Müller survey meter: secondary ionisation is caused by ionized radiation which enters the gas filled chamber, or an electron is produced during the interaction of 
$\mathrm{X}-/ \gamma$ - ray with the wall material [25]

Ionisation chamber: voltage is set up that incident radiation creates only one electronhole-pair which creates a pulse, no further generation of electron-hole-pairs

Silicon photo-diode dosimeters: where holes and electrons are produced by irradiation of the material which leads to a current pulse [26]

MOSFET dosimeters: the irradiation creates electrons and holes which are trapped in the $\mathrm{SiO}_{2}$ which leads to a negative shift in the threshold voltage [26]

Scintillation detectors: scintillation material (crystal, liquid and polymer materials) stops incident radiation and the energy of the particle (e.g. X-ray or $\gamma$-ray (photon), $\alpha$ particle, electron) will be transformed into visible light which will be detected with a photomultiplier tube (PMT) [26]

Thermoluminescence ( $T L$ ) dosimeters: irradiation induces carriers which are trapped until heating the sample (read-out), this leads to recombination of the trapped charges and the emission of light, with the generation of a 'glow curve'

Optically stimulated Luminescence dosimeters (OSLD): same as TLD, the only difference is that trapped carriers are released via stimulation of the sample with light [26, 27]

Diamond detectors: a narrow strip of diamond is doped, which makes the whole diamond stack act as a diode., a charged particle, which passes through these strips, causes ionisation currents, which are detected and measured

It is also important to look at the typical dose ranges, which are applied in radiation dosimetry (see Tab. 2.1). Tab. 2.2 summarise the impact and symptoms of various doses of radiation on the human body. 
Table 2.1: Typical radiation doses applied in dosimetry;

Gy is the unit Gray with $1 \mathrm{~Gy}=1 \mathrm{~J} / \mathrm{kg}$ [14.

\begin{tabular}{|c|c|c|}
\hline Dose & Typical exposure & Typical effect observed \\
\hline $1-5 \mathrm{mGy}$ & One year natural background & Minor risk on biological cells \\
\hline 4 Gy & & $\begin{array}{l}\text { Single exposure is a lethal dose } \\
\text { (leading to death after one month } \\
\text { in } 50 \% \text { of the cases) }\end{array}$ \\
\hline $10-20$ Gy & $\begin{array}{l}\text { Dose delivered to a tumour dur- } \\
\text { ing radiotherapy }\end{array}$ & $\begin{array}{l}\text { Destroys tumour, possibility of } \\
\text { destroying healthy tissue }\end{array}$ \\
\hline 10 - 100 Gy & $\begin{array}{l}\text { Annual dose received by a satel- } \\
\text { lite }\end{array}$ & Damage of electronics \\
\hline 1 MGy & $\begin{array}{l}\text { Dose expected in some detectors } \\
\text { at CERN }\end{array}$ & $\begin{array}{l}\text { Mechanical properties of materi- } \\
\text { als alter }\end{array}$ \\
\hline
\end{tabular}

Table 2.2: Summary of radiation sickness after various doses of radiation (Siev$\mathrm{ert}=\mathrm{Sv}=\mathrm{J} / \mathrm{kg})[15]$.

\begin{tabular}{|l|l|l|}
\hline Dose & Effect & Symptoms \\
\hline \hline $1-5$ mSv per year & & $\begin{array}{l}\text { Average exposure of a citizen during one year } \\
\text { (e.g. as a result of travel by plane, taking } \\
\text { X-rays at the hospital and background radi- } \\
\text { ation) }\end{array}$ \\
\hline up to 0.2 Sv & $\begin{array}{l}\text { Long-term consequences (cancer or modifica- } \\
\text { tion of heritable information) could occur if } \\
\text { the exposure happens over a long period; the } \\
\text { only symptoms are the reduction of the red } \\
\text { blood cells }\end{array}$ \\
\hline
\end{tabular}

Continued on next page 
Table 2.2 - Continued from previous page

\begin{tabular}{|c|c|c|}
\hline Dose & Effect & Symptoms \\
\hline $0.5-1 \mathrm{~Sv}$ & & $\begin{array}{l}\text { Radiation sickness with headaches, exhaus- } \\
\text { tion with a high risk of infection }\end{array}$ \\
\hline $1-2 \mathrm{~Sv}$ & $\begin{array}{l}\text { Light radiation } \\
\text { sickness }\end{array}$ & $\begin{array}{l}10 \% \text { deaths after } 30 \text { days; symptoms dur- } \\
\text { ing } 3-6 \text { hours after irradiation: sickness with } \\
\text { vomiting; symptoms are getting less after a } \\
\text { short recovery but will come back after } 10-14 \\
\text { days: absence of appetite, tiredness, loss of } \\
\text { white blood cells and higher risk of infection }\end{array}$ \\
\hline $2-3 \mathrm{~Sv}$ & $\begin{array}{l}\text { Median radia- } \\
\text { tion sickness }\end{array}$ & $\begin{array}{l}35 \% \text { deaths after } 30 \text { days: after 1- } 6 \text { hours af- } \\
\text { ter irradiation: first symptoms like sickness } \\
\text { with vomiting, short recovery after two days } \\
\text { which will last until one or two weeks, after } \\
\text { recovery again symptoms like illness tired- } \\
\text { ness, loss of hair on the whole body, recov- } \\
\text { ery lasts several months; strong loss of white } \\
\text { blood cells and very high risk of infection }\end{array}$ \\
\hline $3-4 \mathrm{~Sv}$ & $\begin{array}{l}\text { Serious radia- } \\
\text { tion sickness }\end{array}$ & $\begin{array}{l}50 \% \text { deaths after } 30 \text { days: after } 1-6 \text { hours af- } \\
\text { ter the incident: first symptoms like sickness } \\
\text { with vomiting, short recovery after two days } \\
\text { but symptoms come back and additionally } \\
\text { diarrhoea, bleeding in the mouth, under the } \\
\text { skin and in the kidneys; strong loss of white } \\
\text { blood cells and very high risk of infection }\end{array}$ \\
\hline
\end{tabular}

Continued on next page 
Table 2.2 - Continued from previous page

\begin{tabular}{|l|l|l|}
\hline Dose & Effect & Symptoms \\
\hline \hline $4-6 \mathrm{~Sv}$ & Acute radiation & $\begin{array}{l}90 \% \text { deaths after 30 days: symptoms are in } \\
\text { the beginning the same as after the recov- } \\
\text { ery when receiving a dose of 3-4 Sv, only } \\
\text { stronger, death usually appears because of } \\
\text { bleeding and infection after 2-12 days }\end{array}$ \\
\hline 6-10 Sv & $\begin{array}{l}\text { Acute radiation } \\
\text { sickness }\end{array}$ & $\begin{array}{l}\text { after 15-30 minutes after the radiation and } \\
\text { last usually 2 days, a short recovery of 5-10 } \\
\text { days (walking-ghost-phase), after that death } \\
\text { due to circulation collapse }\end{array}$ \\
\hline $20-80 \mathrm{~Sv}$ & $\begin{array}{l}\text { Acute radiation } \\
\text { sickness }\end{array}$ & $\begin{array}{l}100 \% \text { deaths: symptoms same as 6-10 Sv, } \\
\text { rapid death }\end{array}$ \\
\hline
\end{tabular}

In the above Tab. 2.2 the unit Sievert is employed, which is also defined as $1 \mathrm{~Sv}=1 \mathrm{~J} / \mathrm{kg}$ same as the unit Gray $(1 \mathrm{~Gy}=1 \mathrm{~J} / \mathrm{kg}$ ). The difference between these two units is that Gray is utilised to describe a physical quantity. This means it measures amount of energy per mass which is absorbed by an irradiated sample without taking the different types of radiation into account. Different types of radiation have a different effect on the cells even if they transmit the same amount of energy. Therefore, Sievert describes the damage to the cell caused by the different types of radiation. The different factors for the types of radiation are 1 for $\beta$-, $\gamma$ - ray and 5-20 for neutrons, heavy particles and $\alpha$-ray [28].

The purpose of measuring radiation varies and hence, some of the above named dosimeters are not practical to be employed in certain areas. For example ionisation chambers are the gold standard for medical physicists, but they are affected by climatic conditions (ambient pressure and humidity), or they have a dead time after each event during which the system is not able to record another event [25]. 
Scintillation detectors have the problem of quenching (optically or chemically quenching). Quenching is an effect where the light output is reduced by re-absorption of the scintillation light by a scintillation ion or by light scattering. These detectors are also affected by Cherenkov radiation, which means when electromagnetic radiation is emitted after a charged particle is passing through a dielectric medium with a speed greater than the phase velocity of light in that material. The charged particles polarise the molecules of the detector material due to rapid return to their ground state and radiation is emitted in the process [25, 26].

Diodes have the disadvantage that the material is not tissue equivalent, and the ambient temperature is important during measurement. The diodes only detect the radiation of the field, which will be used for linear accelerators or imaging plates in the hospital and not the dose.

In TLD and OSLD, the radiation induces carriers, which are trapped until a read out. During this read-out, the carriers are excited out of their traps and emit photons (luminescence) [29]. The emitted light is detected with a photomultiplier tube (PMT) and is a value for the absorbed radiation dose of the crystal [27]. So far, there is only one company which produces a commercial $\mathrm{Al}_{2} \mathrm{O}_{3}$ :C OSL dosimeter (OSLD). This company is Landauer $\AA$ with their Luxel $\AA+$ Dosimeter. The Luxel@ + detects X-, $\beta$-, $\gamma$ - and neutron radiation [30]. Commercial OSL dosimeters are capable of measurements as low as $50 \mu \mathrm{Gy}$. McKeever also reported they can measure as low as $10 \mu$ Gy. Both techniques of TL and OSL have advantages and disadvantages - optically stimulated luminescence dosimeters have the biggest advantage of a nearly real time read-out. OSL has been employed for personal dosimetry as well as the determination of environmental radiation doses absorbed by geological materials in order to date them. The OSL technique in personal dosimeters promises high sensitivity, rapid read-out, automation of read-out, good fading characteristics, and the material should have an effective atomic number like that of tissue (that means the material response to radiation is like that of tissue) [31, 32]. A tissue equivalent response to radiation is important, especially if the radiation energy of the $\mathrm{X}$ - or $\gamma$ - rays is 
between $20 \mathrm{keV}$ and $\sim 100 \mathrm{keV}$. In this region the photoelectric interaction is predominant (as shown in Fig. 2.1), which is a function of the third power of the atomic number and the linear attenuation coefficient for this region is given by,

$$
\mu_{p}=\rho_{e} C_{P} \frac{Z^{m}}{E^{n}}
$$

where components have been determined experimental, $\mathrm{m}=3.8, \mathrm{n}=3.2$ [33], $\rho$ is the density of the electrons, $C_{p}$ is an empirical constant and is $9.8 \times 10^{-24}$ [34], $Z$ is the atomic number of the detector material and $E$ the energy of the electrons. For detector materials with a higher atomic number than tissue there will be an overresponse in the mass energy absorption coefficient for energies up to $100 \mathrm{keV}$ and the measurements will be dependent of the incident energy of the photon. For materials with a similar atomic number to tissue, the measurements will become independent of the incident energy of the photon (explained in a later chapter). There is still a limitation of employing materials with an overresponse below $100 \mathrm{keV}$. Even if the average photon energy is above $100 \mathrm{keV}$ secondary electrons with an energy below $100 \mathrm{keV}$ will be produced. In a bremsstrahlung photon beam with a peak energy above $100 \mathrm{keV}$ there are still some photons with a lower energy than 100 $\mathrm{keV}$ and this causes an overresponse because the measurements below $100 \mathrm{keV}$ are not independent of the photon energy [35]. 


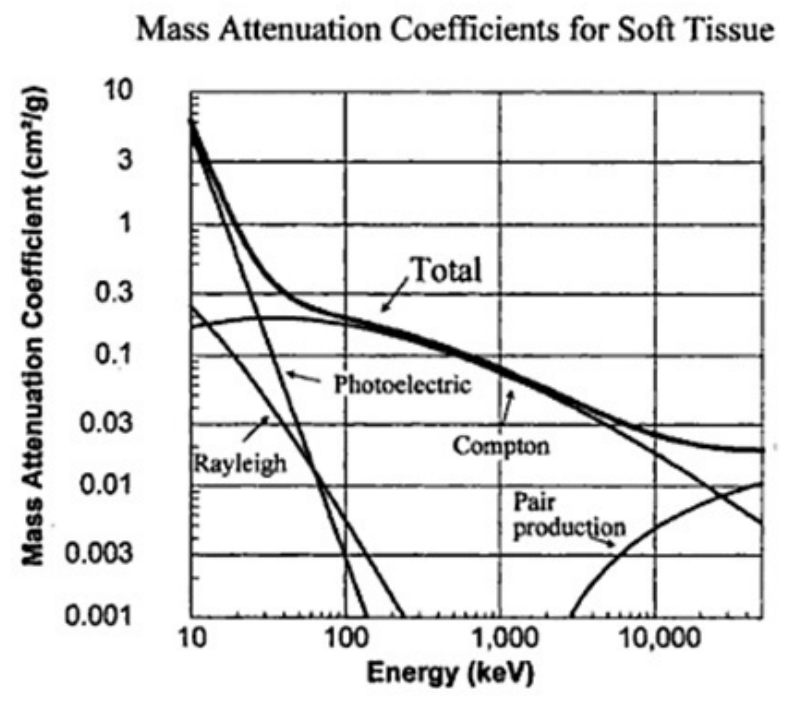

Figure 2.1: Graph of the Rayleigh, photoelectric, Compton, pair production, and total mass attenuation coefficient for soft tissue $(Z=7)$ as a function of energy [1].

OSL dosimeters constructed of fluoroperovskite compounds as will be explained in this thesis, have the advantage of multiple read-out or the same event [31] as well as the detection of the absorbed dose and the dose rate of an event. In general, the detectors can be produced in a smaller size compared to the ion chambers, which are mainly utilised at the hospital these days. With these materials, it is possible to shift the emission to high wavelengths and therefore Cherenkov radiation is not an issue anymore. Furthermore, large area detectors can be produced to "image" the radiation field [27]. OSL dosimeters can also be combined with radioluminescence dosimeters (RLD) [23, 24, 36]. The bulk materials which are observed in this thesis show OSL [3] and RL [10, 11, 12]. RLD have the advantage for real-time read-out, which means the absorbed dose in the material can be measured as a function of the intensity of the emitted light. This application is important for dosimeters employed for patients in a hospital, i.e.. The medical physicist is able to immediately stop a treatment if there is an over or under dose. 


\subsection{Radiometric quantities}

To describe a radiation field, which includes all types of radiations, one can utilise the particle fluence, $\Phi$, which is the quotient of the number of the particles $\delta N$ incident on cross-sectional area $\delta a$,

$$
\Phi=\frac{\delta N}{\delta a} \quad\left(\text { unit } \mathrm{m}^{-2}\right)
$$

With the corresponding fluence rate of particles, $\phi$,

$$
\phi=\frac{\delta^{2} N}{\delta a \delta t} \quad\left(\text { unit } \mathrm{m}^{-2} \mathrm{~s}^{-1}\right)
$$

The same applies for the energy fluence, $\Psi$, and energy fluence rate, $\psi$,

$$
\Psi=\frac{\delta R}{\delta a} \quad\left(\text { unit } \mathrm{Jm}^{-2}\right)
$$

With $\delta R$ being the radiant energy emitted, transferred or received on a cross-sectional area $\delta a$ [3, 37.

$$
\psi=\frac{\delta^{2} R}{\delta a \delta t} \quad\left(\text { unit } \mathrm{Wm}^{-2}\right)
$$

\subsection{Interaction of radiation with matter and their energy dependence}

\subsubsection{Interaction of radiation with matter}

The interaction of charged and uncharged particles are determined by measuring the cross section $\sigma$, which is usually characterised in the unit barn $\left(1 \mathrm{~b}=10^{-24} \mathrm{~cm}^{2}=10^{-28} \mathrm{~m}^{2}\right)$. The sum of all the cross sections $\sigma_{J}$ of each particle $J$ multiplied with the quotient of the Avogadro constant $N_{A}$ and the molecular mass $M$ of the target material is equal to the quotient between the linear attenuation coefficient $\mu$ and the density of the absorbing material $\rho$ [38].

$$
\frac{d N}{d l}=N \mu
$$




$$
\frac{\mu}{\rho}=\frac{1}{\rho d l} \frac{d N}{N}=\frac{N_{A}}{M} \sum_{J} \sigma_{J} \quad\left(\text { unit } \mathrm{m}^{2} \mathrm{~kg}^{-1}\right)
$$

$d N / N$ is the number of particles, which interact with other particles during their transit with a distance $d l$. In the following equation 2.9 , the mass energy transfer coefficient is shown. $\delta R_{t r} / R$ is the amount of incident energy that is transferred as kinetic energy by the charged particles during interaction in the distance $d l$. The kinetic energy is converted from the radiation energy of these particles. $\mu_{t r} / \rho$ includes the energy loss caused by radiative processes.

$$
\begin{gathered}
\frac{d R_{t r}}{d l}=\mu_{t r} R_{t r} \\
\frac{\mu_{t r}}{\rho}=\frac{1}{\rho \delta l} \frac{\delta R_{t r}}{R} \quad\left(\text { unit } \mathrm{m}^{2} \mathrm{~kg}^{-1}\right)
\end{gathered}
$$

The mass energy absorption coefficient is more a quantity for dosimetric calculations. The linear attenuation coefficient $\mu / \rho$ describes the total energy, which is absorbed by the material. The mass energy transfer coefficient $\mu_{t r} / \rho$ presents a value of the radiation caused from secondary electrons, and describes the biological damage caused in the material.

$$
\frac{\mu_{e n}}{\rho}=\frac{\mu_{t r}}{\rho}(1-g) \quad\left(\text { unit } \mathrm{m}^{2} \mathrm{~kg}^{-1}\right)
$$

In this equation 2.10, $g$ represents the energy loss during the radiative process. Equation 2.10 changes for compounds as a fact of the independence of the components.

$$
\left(\frac{\mu_{e n}}{\rho}\right)_{\text {compound }}=\sum_{i} w_{i}\left(\frac{\mu_{e n}}{\rho}\right)_{i} \quad .
$$

The corresponding mass energy absorption cross section $\sigma_{e n}$ is,

$$
\left(\sigma_{\text {en }}\right)_{\text {compound }}=\frac{\left(\mu_{\text {en }} / \rho\right)_{\text {compound }}}{N_{A} \sum_{i} w_{i} / A_{i}}
$$

with,

$$
w_{i}=\frac{A_{i}}{\sum_{j} A_{j}}
$$

as the fraction of the abundance by weight and $A_{i}$ the atomic weight of the $i^{\text {th }}$ component (equation 2.12). For charged particles, the mass stopping power is expressed by following equation 2.14 ,

$$
\frac{S}{\rho}=\frac{1}{\rho} \frac{\delta E}{\delta l} \quad\left(\text { unit } \mathrm{Jm}^{2} \mathrm{~kg}^{-1} \text { or } \mathrm{eVm}^{2} \mathrm{~kg}^{-1}\right)
$$


which describes the energy loss $\delta E$ of a charged particle during a distance $\delta l[3]$.

The restricted linear collision stopping power defines the energy loss $\left(d E_{\Delta}\right)$ of a charged particle during soft and hard collision along a distance $d l$ minus the total kinetic energy of charged particles that are released with an excess kinetic energy of the value $\Delta$.

$$
L_{\Delta}=\frac{d E_{\Delta}}{d l} \quad\left(\text { unit } \mathrm{Jm}^{-1}=\mathrm{eVm}^{-1}\right)
$$

By dividing $L_{\Delta}$ by the density of the material, the restricted mass collision stopping power is defined. The sum of the collision mass stopping power and the radiative mass stopping power is the total mass stopping power. In Fig. 2.2, the total unrestricted and restricted electron mass stopping power of carbon is shown [2].

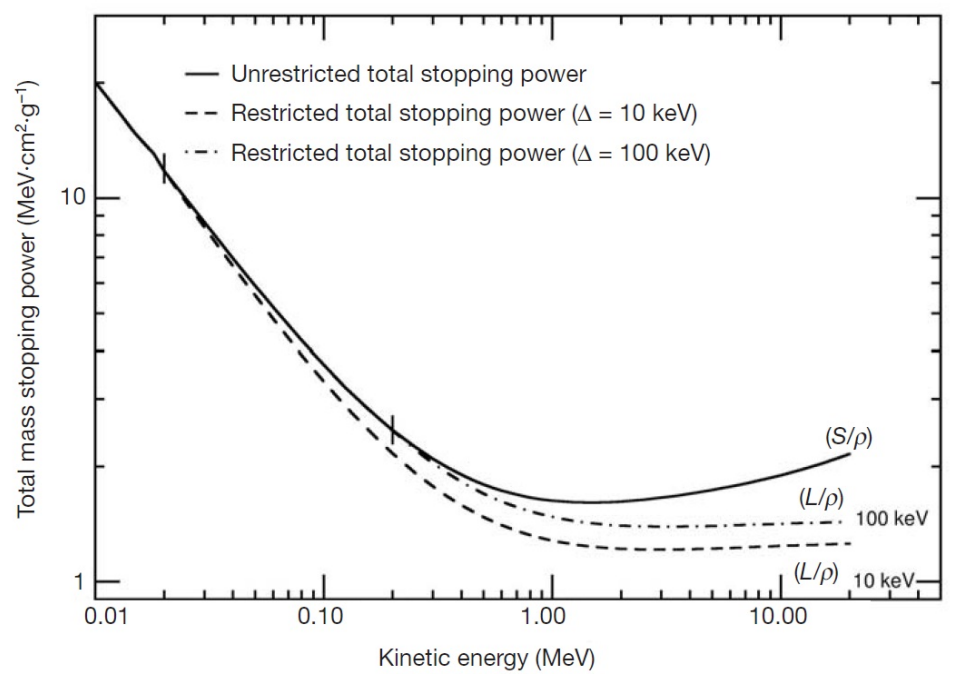

Figure 2.2: Unrestricted $\mathrm{S} / \rho$ and restricted $\left((\mathrm{L} / \rho)_{\Delta}\right.$ with $\Delta=10$ and $\left.100 \mathrm{keV}\right)$ total mass stopping powers for carbon $\left(\rho=1.70 \mathrm{~g} / \mathrm{cm}^{3}\right)$. Vertical lines indicate the points at which restricted and unrestricted mass stopping powers begin to diverge as the kinetic energy increases [2].

\subsubsection{Energy dependence of a radiative process}

For personal dosimetry or radiotherapy, it is essential that the materials show tissue equivalence. Therefore, the main focus in today's research is to find materials, which have the 
same absorption cross-section as human tissue has. This chapter will focus on the considerations, which have to be taken into account for the design of a detector of a dosimeter with these properties. In Fig. 2.3, the mass energy absorption coefficient for tissue and common detector materials are illustrated.

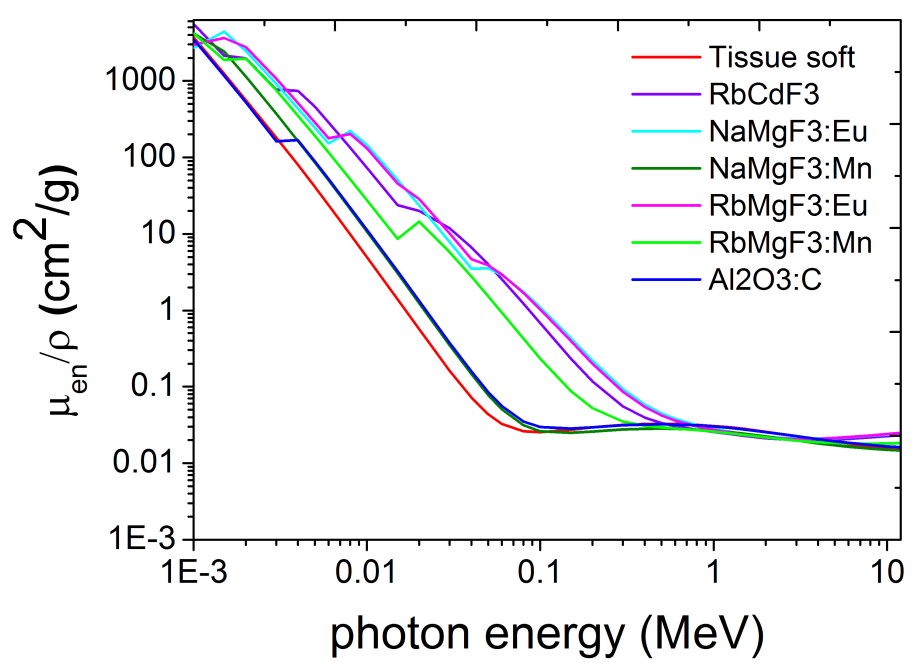

Figure 2.3: Mass energy absorption coefficients for detector materials.

The photons usually have energies in the range between several hundred eV (soft X-rays) to several $\mathrm{MeV}$ (hard X-rays) and can be up to $20 \mathrm{MeV}$ for photon radiotherapy. Radiation defects are usually calculated by the mass-energy-absorption, which was expressed in equation 2.10. In tissue, the photoelectric contribution is large at lower energies and at these energies the photon energy becomes dependent on $\sim Z^{4} / E^{3}$. This effect is dependent on the atomic number of the material and therefore, the cross over energy from the photoelectric to the Compton contribution increases with increasing Z. The probability for the Compton effect is independent of the atomic number of the material; it is only an interaction with the electrons of the absorber. The energy of the emitted photon is dependent on the angle of the emission and on the incident photon energy. The energy dependence $E D$ for a dosimeter material to the photon energy $E$ is shown in the following equation 2.16 in the large detector limit, which means equation 2.16 is not valid for finite 
sized detectors, which is explained in the Burlin cavity theory (as explained later in this chapter) [1, 2, 39].

$$
E D=\frac{\left(\mu_{\text {en }}(E) / \rho\right)_{\text {detector }}}{\left(\mu_{\text {en }}(E) / \rho\right)_{\text {reference }}} / \frac{\left(\mu_{\text {en }}\left(E_{0}\right) / \rho\right)_{\text {detector }}}{\left(\mu_{\text {en }}\left(E_{0}\right) / \rho\right)_{\text {reference }}}
$$

Furthermore, it is assumed that the energies are low enough so that thermal neutron production can be ignored (below $\sim 10 \mathrm{MeV}$ ). The mass energy absorption coefficient of the detector and the reference material are given by $\left(\mu_{e n} / \rho\right)_{\text {detector }}$ and $\left(\mu_{e n} / \rho\right)_{\text {reference }}$, respectively, and $\mathrm{E}_{0}$ is the photon energy of a reference radiation. $E D$ values are shown in Fig. 2.4 for some detector materials.

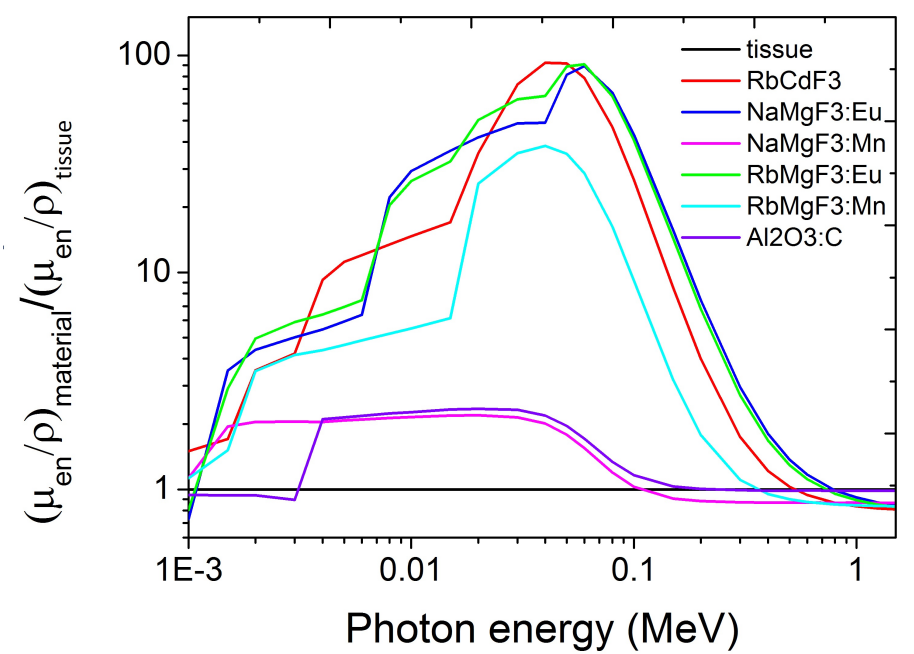

Figure 2.4: Energy response of detector materials compared to tissue.

With $s_{d}$ and $s_{m}$ expressing the mass collision stopping powers of the detector and the medium, respectively. The quotient of the absorbed dose in the detector and the surrounding medium is [40],

$$
\frac{D_{d}}{D_{m}}=f=d \frac{\overline{s_{d}}}{s_{m}}+(1-d) \frac{\left(\mu_{e n} / \rho\right)_{d}}{\left(\mu_{e n} / \rho\right)_{m}}
$$

$d$ is expressed in the following equation 2.18, $d$ is also related to the size of the cavity and it becomes zero for large cavities and approaches unity for small ones. As a result of this, equation 2.17 must be considered for energies above $\sim 1 \mathrm{MeV}$ as the cavity behaves as an 
intermediate cavity, and the Burlin cavity theory needs to be applied and small cavity size is only obtained for energies above $20 \mathrm{MeV}$ (see also Fig. 2.5) [1],

$$
d=\frac{1-e^{-\beta g}}{\beta g}
$$

with $\beta$ the effective mass attenuation coefficient for electrons and $g$ the average path length. For a small sized cavity, as explained in the Bragg-Gray cavity theory, the quotient of the average absorbed dose in the cavity and the absorbed dose in the medium around the scintillator (without the scintillator) is equal to the quotient of the average mass collision stopping powers of the cavity and the medium. For large cavities, the quotient of the doses is equal to the quotient of the average mass-energy-absorption coefficients of the cavity and the medium. In the latter case, it is assumed that charged particles are completely generated by photon interactions in the cavity and the energy of the particles is absorbed in the cavity. Burlin explains the gap between these two other theories [1].

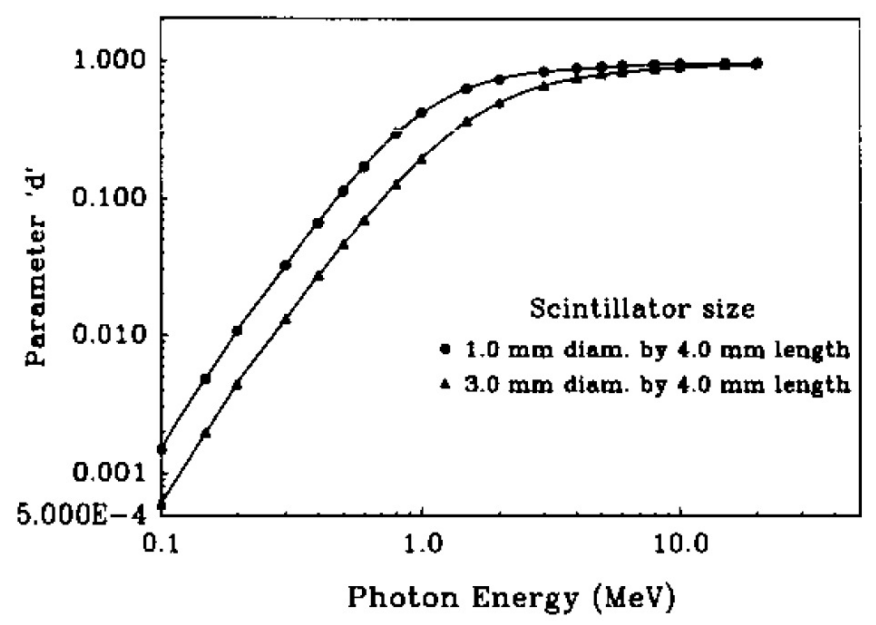

Figure 2.5: Burlin cavity theory parameter $d$ as a function of photon energy for two cylindrical plastic scintillator sizes. This parameter represents the fraction of the dose deposited in the cavity due to electrons generated outside the cavity [1].

The energy response is then expressed by equation 2.19 .

$$
E D=\frac{D_{d}(E)}{D_{m}(E)} / \frac{D_{d}\left(E_{0}\right)}{D_{m}\left(E_{0}\right)}
$$


Important for this work is the energy dependence of the fluoroperovskites, especially $\mathrm{RbMgF}_{3}$ and $\mathrm{NaMgF}_{3}$, which will be mainly investigated [3].

\subsubsection{Effective atomic number}

The mass energy absorption cross section defines the interactions caused by the radiation. For photon energies below $1 \mathrm{MeV}$, the photoelectric effect is dependent on the effective atomic number of the material. The effective atomic number calculates the average atomic number of a compound. Hence, the effective atomic number is equivalent to the atomic number $Z$ of an element, which has the same cross section as the compound. In cases for photon energies below $1 \mathrm{MeV}$, the energy response of a compound with one number is employed to describe the cross section rather than using the whole $E D$ curve. Therefore, a low energy approximation to the photoelectric cross section for the effective atomic number is presented by the following equation [41],

$$
Z_{e f f_{E M P}}=\sqrt[3]{\frac{\sum n_{i} Z_{i}^{4}}{\sum n_{i} Z_{i}}}
$$

with $n_{i}$ as the molar fraction and $Z_{i}$ the atomic number of the $i^{\text {th }}$ component. If the material has a high atomic number the value will be overestimated. Tissue has a value of 7.62 calculated with the equation described above [3]. A material is considered to be useful for medical applications with a $\mathrm{Z}_{\text {eff }}<14$. The effective atomic numbers of some of our materials are given below:

$\mathrm{Z}_{e f f}=10.8$ for $\mathrm{NaMgF}_{3}: \mathrm{Mn}^{2+}$

$\mathrm{Z}_{e f f}=13.6$ for $\mathrm{NaMgF}_{3}: \mathrm{Eu}^{2+}$

$\mathrm{Z}_{\text {eff }}=31.3$ for $\mathrm{RbMgF}_{3}: \mathrm{Mn}^{2+}$

$\mathrm{Z}_{e f f}=31.7$ for $\mathrm{RbMgF}_{3}: \mathrm{Eu}^{2+}$ 


\subsubsection{Radiation protection dosimetry}

To determine the effect of absorbed radiation on a biological cell, the quality factor $Q$ has been introduced. The quantity factor considers the different types of radiation. The product of the quality factor $Q$ and the absorbed dose $D$ is the dose equivalent $H$ [37, 42].

$$
H=Q D \quad\left(\text { unit } \mathrm{Jkg}^{-1}\right)
$$

The dependence of the quality factor $\mathrm{Q}$ on the linear energy transfer $\mathrm{L}$ in water has been specified by the ICRP.

$$
Q(L)= \begin{cases}1 & \text { for } L \leq 10 \\ 0.32 L-2.2 & \text { for } 10<L<100 \\ 300 / \sqrt{L} & \text { for } L \geq 100\end{cases}
$$

$L$ has the unit $\mathrm{keV} / \mu \mathrm{m}[37,42]$.

\subsection{Dosimetric quantities}

Kerma means kinetic energy released per unit mass and describes the sum of the kinetic energy $\left(d E_{t r}\right)$ of charged particles, which is transferred to uncharged particles in a mass $d m$ [3, 37, 43].

$$
K=\frac{d E_{t r}}{d m}=\int \Phi_{E} E \frac{\mu_{t r}}{\rho} d E \quad\left(\text { unit } \mathrm{Gy}=\mathrm{Jkg}^{-1}\right)
$$

Where $\Phi_{E}=\mathrm{d} \Phi / \mathrm{dE}$ is a given particle fluence energy distribution and Gy is the unit Gray with,

$$
1 \mathrm{~Gy}=1 \frac{J}{k g}=100 \mathrm{rad}
$$

The corresponding kerma rate is,

$$
\dot{K}=\frac{d K}{d t} \quad\left(\text { unit } \mathrm{Gys}^{-1}=\mathrm{Wkg}^{-1}\right)
$$


The absorbed dose $D$ is the quotient of the mean energy imparted $d \bar{\epsilon}$ to a mass $d m$.

$$
D=\frac{\bar{\epsilon}}{d m} \quad\left(\text { unit } \mathrm{Gy}=\mathrm{Jkg}^{-1}\right)
$$

With the corresponding dose rate,

$$
\dot{D}=\frac{d D}{d t} \quad\left(\text { unit } \mathrm{Gys}^{-1}=\mathrm{Wkg}^{-1}\right)
$$

Kerma and the absorbed dose have the same units but kerma is a microscopic quantity, which describes the radiation field, and the absorbed dose is a macroscopic quantity [3, 37, 43].

\subsection{Fields of dosimetry}

\subsubsection{Personal dosimetry}

The aim of implementing personal dosimeters is to monitor the radiation for employees exposed to radiation, i.e. in radiology, at nuclear power plants, radiation therapy or teaching facilities like universities. As mentioned in the introduction section, it is important that the dosimeter has a nearly tissue equivalent energy dependence. Therefore, the TL dosimeters (TLD) are to be employed in this field, but also film badge, scintillators, OSLD, or diodes for real-time measurements [6].

To monitor the penetration of radiation of an area, the ambient dose equivalent $H^{*}(d)$, with $\mathrm{d}=10 \mathrm{~mm}$, is utilised. In this case, $\mathrm{H}^{*}(\mathrm{~d})$ is also written as $H^{*}(10)$ and the value should be independent of the directional distribution of the dosimeter [44, 45]. For monitoring a low-penetration of radiation, the directional dose equivalent $H^{\prime}(d, \Omega)$, with $\mathrm{d}=0.07$ $\mathrm{mm}$ or $\mathrm{d}=3 \mathrm{~mm}$, is utilised. The directional dose equivalent is the dose which is to be measured in an actual radiation field with $\Omega$ as the specific direction of the field at a depth d. $H^{\prime}(3, \Omega)$ is to be chosen when monitoring the radiation to the eye lens, but in almost all cases $H^{\prime}(0.07, \Omega)$ is employed for monitoring low- penetration of radiation [44, 45]. 
$H_{p}(d)$ is the personal dose equivalent, which considers the dose equivalent in tissue at a depth $d$ in a human body where the actual personal dosimeter should be worn. In this case, $H_{p}(0.07)$ and $H_{p}(10)$ are defined as the skin dose at a depth of $\mathrm{d}=0.07 \mathrm{~mm}$ and the effective dose at $\mathrm{d}=10 \mathrm{~mm}$ in the body, respectively. However, they are to be measured by the dosimeter wearing on the body. $H_{p}(3)$ is employed in rare cases as the dose to the lens of an eye at a depth of $\mathrm{d}=3 \mathrm{~mm}$. These values are defined for all types of radiation, and also include the backscattering of the body [44, 45].

\subsubsection{Medical dosimetry}

Radiation is utilised in two main areas in medicine: One is radiation imaging (i.e. computed tomography (CT), projection radiography, mammography) and the other is radiotherapy (i.e. external beam radiotherapy, brachytherapy and systemic radioisotope therapy). For over 120 years, radiation imaging has been employed to examine the whole body or parts of it for clinical diagnostics. These imaging processes have since been improved and the doses have been optimised to reduce the harm of radiation to biological cells. In radiation therapy, the property of ionising radiation to damage human tissue cells is utilised to treat cancer. Compared to medical imaging a higher dose is exposed to the body during radiation therapy. This implies different requirements for dosimeters between these regimes.

\section{Medical imaging}

There are several methods for medical imaging including X-ray examinations, fluoroscopy, $\mathrm{CT}$ and mammography. The dose exposed to the body is measured in mSv and various doses are employed within the different methods [46, 47, 48].

X-ray examination or projection radiography is a technique where X-rays are transmitted through the body and then are captured in a plate of sensors to produce an image. On the other hand, fluoroscopy is a method to produce real-time images during a procedure. 
Therefore, it needs a constant input of X-rays and works with contrast media to visualise the organs as they work [15, 16, 17].

CT or X-ray computed tomography is employed to create a 3D image of the body. 3D data of the patient anatomy is produced via reconstruction of many beamlets or rays $(2 \mathrm{D}$ slides) [15, 16, 17].

Mammography is usually utilised for breast screening to detect breast cancer. The mechanism is similar to X-ray examination but it uses lower doses than CT [15, 16, 17].

In Tab. 2.3, the different methods of radiation imaging with their various scan doses are listed.

Table 2.3: Typically effective doses applied during medical examination [15, 16, 17].

\begin{tabular}{|l|l|}
\hline Type of examination & Typically effective dose (mSv) \\
\hline \hline Intraoral X-ray (dental) & 0.005 \\
\hline Chest X-ray & 0.1 \\
\hline Mammography & 0.4 \\
\hline Head CT & 1.5 \\
\hline Chest CT (low dose) & 1.5 \\
\hline Screening mammography & 3 \\
\hline Abdomen CT & 5.3 \\
\hline Chest CT & 7 \\
\hline Chest, abdomen and pelvis CT & 9.9 \\
\hline CT-abdomen and pelvis & 15 \\
\hline Coronary CT angiography (CTA-heart) & 16 \\
\hline Fluoroscopy & 0.9 \\
\hline
\end{tabular}

The natural background radiation to a person is around 1-5 mSv per year. 
To measure the dose received to the patient, measurements are usually performed free-inair, on phantoms or directly on the patients with TL dosimeters. To protect the patient from the harm from the radiation during this diagnostic process, there is a demand for tissue equivalent dosimeters, which measure the absorbed dose to the patient in real time [3].

\section{Radiation therapy}

Radiation therapy is a very complex area and medical physicists in the hospital need special education and extensive experience before becoming a certified medical physicist. In general, there are three different methods of radiation therapy, which will be briefly explained in the following section.

External beam radiotherapy employs a linear accelerator to produce 2D cross section beams. By control of couch, collimator, and gantry angles, the patient can be irradiated from any given angle. Dosimeters are needed for dose verification and validation to check in vivo dose to the patient or the treatment plan prior to radiation. Currently, diodes and TL, OSL dosimeters are utilised in this area.

Another way to treat the cancer is to place radiation source(s) proximal to the tumour, which is applied in brachytherapy. For this, the source(s) may be applied as a permanent implant, or delivered from an afterloader for a temporary exposure. This means, there can be a higher radiation dose applied to the cancer cells while healthy tissue remains minimally affected [15, 16, 17].

For some techniques, the cancer is targeted via a radioactive isotope that is ingested or infused to the bloodstream [15, 16, 17]. The dose applied to cancer during radiation therapy also depends on the type and stage of the cancer. Tab. 2.4 shows some typical doses applied in photon radiation therapy [15, 16, 17]. 
Table 2.4: Types of cancer treatment with typically applied doses (in Gy) [15, 16, 17].

\begin{tabular}{|l|l|}
\hline Type of treatment & Applied dose (Gy) \\
\hline \hline Curative & $60-80$ \\
\hline Lymphomas (curative) & $20-40$ \\
\hline Preventive (adjuvant) & $45-60$ \\
\hline
\end{tabular}

The dose to treat the cancer is fractionated. This means, the total dose is not applied all at once. Healthy tissue exhibits better repair mechanisms, so fractionation allows the healthy cells time to recover while the more radiosensitive tumour cells does not. The dose applied to the cancer also depends on the specific radiosensitivity of the cancer. For example, leukaemia, most lymphomas or germ cell tumours are highly radiosensitive when a lower dose is applied. Most of the epithelial cancers are more radiosensitive, and usually a dose up to 60-70 Gy is applied. Radiosensitive are renal cells cancer and melanoma [15, 16, 17].

Currently, the absorbed dose is calculated before the treatment and any required measurements are carried out on a phantom. For complex techniques, medical physicists are interested in measuring the dose received by the patient in real time as well as new methods for dose verification and validation. Time-resolved measurements can better indicate where delivery issues exist. Measurement methods, which have been employed so far involve are semiconductor dosimeters, TLD and OSL dosimeters [49, 50, 51. Most of the detectors do not demonstrate tissue equivalence nor provide ideal spatial resolution (e.g. ionisation chambers) for the new treatment regimes. TLD does not provide real time dose information but as they are non-invasive, they are widely used. Therefore, there is a need for small, tissue-equivalent detectors to measure the actual absorbed dose on the patient in real time. Currently, plastic scintillators are the only commercial provided OSL dosimeter is used to measure the actual dose received by the patient [3, 52]. 


\subsubsection{Environmental dosimetry}

Environmental radiation means, the radiation a person is receiving through cosmic radiation, terrestrial radiation (natural radionuclide in the earth crust) and radiation from man-made radiation (medical treatments, radiation sources being moved, nuclear power plants, coal-fired power plants and nuclear weapons testing i.e.). Therefore, environmental dosimetry measures the exposure of radiation by nuclear and radiological facilities to the environment. A high sensitivity for dosimeters is required (monthly radiation levels around 40-80 $\mu \mathrm{Gy}$ ), as well as robustness and insensitivity to extreme environmental conditions (humidity, temperature, mechanical stress). TL dosimeters are mostly to be employed in this area of application [53, 54, 55].

\subsubsection{Retrospective dosimetry}

Retrospective dosimetry monitors radiation caused by human factors e.g. by nuclear power plant accidents. A sample of an inorganic material is collected and measured with TL, OSL dosimeters or ESR (electron spin resonance) [56]. The same techniques are utilised for geological or archaeologically dating. Requirements for the detector should be an equal effective atomic number as the investigated material. As mentioned in the previous section, the measured dosed are quite low and therefore a high sensitivity is needed [56]. 


\section{Theoretical Background}

This chapter outlines a theoretical overview of the materials to be investigated, explains the principles of the techniques and equipment employed to measure the properties of the samples.

\subsection{Fluoroperovskite}

Fluoroperovskites are part of the group of the perovskites. A perovskite structure is any material with the same type of crystal structure as calcium titanium oxide $\left(\mathrm{CaTiO}_{3}\right)$. The fluoroperovskites have the structure $\mathrm{ABF}_{3}$, where $\mathrm{A}$ is a cation, $\mathrm{B}$ a metal (in this case $\mathrm{Mg}$ ) and $\mathrm{F}$ is the chemical symbol of fluorine. These structures have been recently of great interest for their range of electronic, magnetic and optical properties. The crystal structure of the fluoroperovskites would be ideally cubic. However, due to the small size of the cation the structure changes and produces a lower symmetry. The undistorted structure of the perovskite is cubic $(=1)$. The degree of distortion of the cubic structure can be estimated from the tolerance factor $t$ by Goldschmidt [57],

$$
t=\frac{r_{A}+r_{F}}{\sqrt{2}\left(r_{B}+r_{F}\right)}
$$

where $\mathrm{r}_{A}$ is the radius of the cation, $\mathrm{r}_{F}$ the radius of fluorine and $\mathrm{r}_{B}$ the radius of the metal. From $0.76 \leq t \leq 0.88$ the structure of the crystal will be orthorhombic, like $\mathrm{GdFeO}_{3}$, and $0.89 \leq t \leq 1.02$ cubic structures are expected, like $\mathrm{BaTiO}_{3}$, and $>1$ hexagonal structures 
are expected, like $\mathrm{BaNiO}_{3}$ [57]. $\mathrm{NaMgF}_{3}$ has a Goldschmidt factor of 0.83 and the structure is orthorhombic with the space group of $\mathrm{P}_{b n m}$. In this case, Eu substitutes for $\mathrm{Na}^{+}$and $\mathrm{Mn}^{2+}$ for $\mathrm{Mg}^{2+}$. For $\mathrm{RbMgF}_{3}$ the Goldschmidt factor is 1.02 and the structure is therefore hexagonal with a space group of $\mathrm{P}_{6_{3} / m m c}$. Eu substitutes for $\mathrm{Rb}^{+}$and $\mathrm{Mn}^{2+}$ for $\mathrm{Mg}^{2+}$, see Fig. 3.1 .

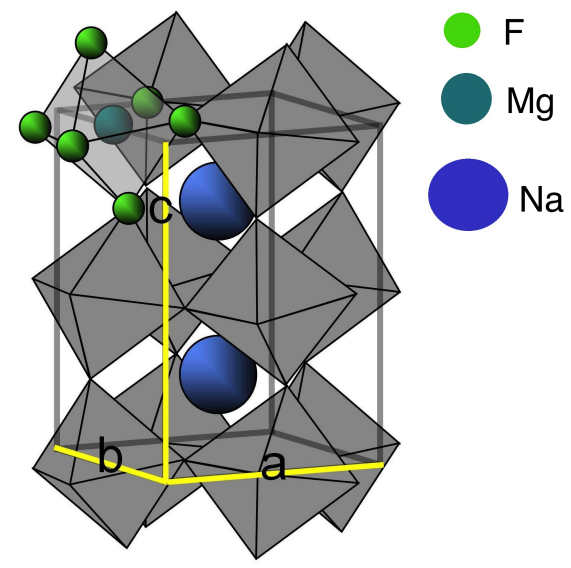

(a)

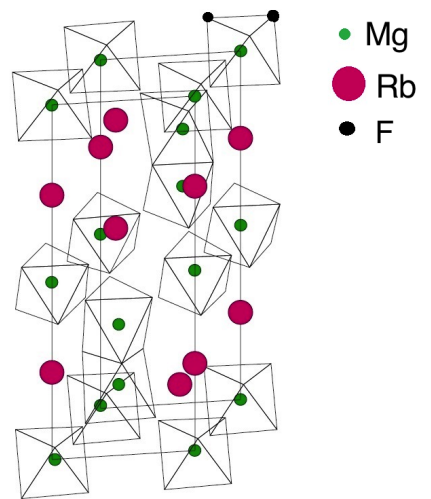

(b)

Figure 3.1: (a) Orthorhombic structure of $\mathrm{NaMgF}_{3}$ and

(b) Hexagonal structure of $\mathrm{RbMgF}_{3}[3]$.

\subsection{Photoluminescence}

The excitation of an electron to a higher energy state due to the absorption of a photon of a certain energy and the decay to a lower energy state while emitting a photon is called photoluminescence $(\mathrm{PL})$. The wavelength of the emitted photon and the wavelength of the photon to excite the material are collected and from this information the structure of the material will be obtained.

In this thesis, spectra of rare earth (RE) ions within the host crystal will be observed. No PL was detected from undoped samples. RE show the presence of deep $4 \mathrm{f}$ shell, which is not completely filled and electrons in this shell are shielded by electrons in an outer shell. 
Hence, their emission will result in a sharp line spectra. There is a difference between the emission of a free RE and RE in solids, because the transition takes place within the $4 \mathrm{f}$ shell in the crystal. Therefore, the absorption and emission takes place between different levels, and this results in a shift in the spectral position compared to the free RE ions. This shift is called "Stokes Shift", which is illustrated in Fig. 3.2 in a configuration coordinate diagram. In Fig. 3.2, $\mathrm{E}$ is the energy of the levels and $\mathrm{Q}$ is the configuration coordinate.

In the process of PL, an absorption of a photon takes place and an electron gets excited from a ground state to a higher excited state. This excitation is too fast and hence the lattice does not have time to relax. Therefore, multi phonon emissions take place and the electron moves into a lower excited state while loosing energy to the lattice. The electron emits a photon while going back to the ground and this photon has less energy as the absorbed photon ("Stokes Shift") [18].

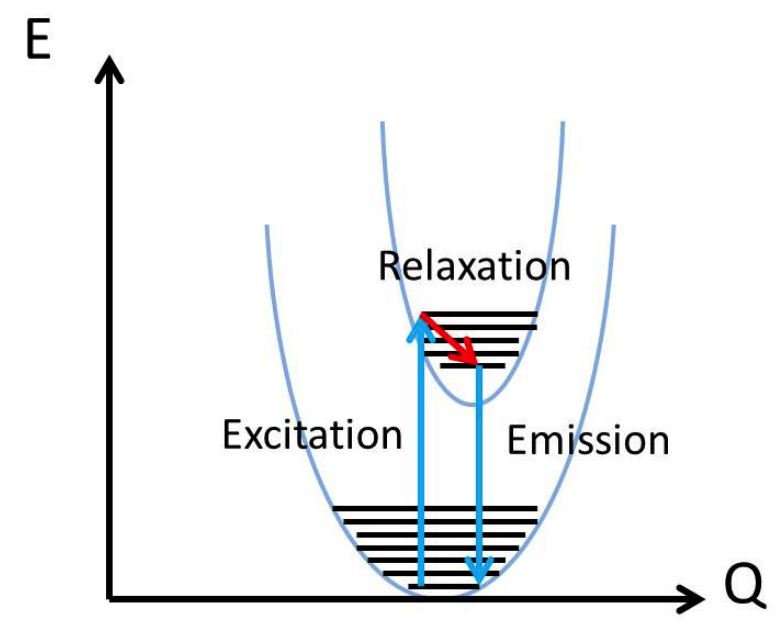

Figure 3.2: Photoluminescence process.

Electric dipole transitions within a $4 \mathrm{f}$ shell are forbidden in a free ion because the parity does not change within the shell, but they are allowed for magnetic dipole transitions. Therefore, it is expected that only magnetic dipole transitions will occur. However, for an ion in a crystal there can be a mixture of the $4 \mathrm{f}^{n}$ states with opposite parity states of 
the $4 \mathrm{f}^{n}-5 \mathrm{~d}$ configuration due to an odd-parity crystal field. The magnetic dipole process is five orders of magnitude smaller than the electric dipole one for a free ion. This means, in a crystal even a small amount of this crystal field mixing can result in the majority of the intensity arising from this "forced" or "induced" electric dipole transition. Transitions within the $4 \mathrm{f}$ shell can also become allowed through mechanism such as the coupling of $4 \mathrm{f}$ electrons with transient dipoles, which are induced in the ligands by the radiation field, leading to an increase in intensity of even parity transitions. These transitions are called "hypersensitive" transitions because they show large variations in oscillator strengths depending on the surrounding environment. These transition are discussed in more detail by Görller-Walrand and Binnemans, but are also known as "pseudo-quadrupole" transitions because the relevant selection rules follow those of true quadrupole transitions [58]. Selection rules for all these processes are listed in Tab. 3.1 and are assumptions for the Judd-Ofelt theory summarised by Walsh e.g. [18, 19, 20].

Table 3.1: Selection rules for the forced electric dipole process, magnetic dipole process and pseudo electric quadrupole transition [18, 19, 20].

\begin{tabular}{|l|l|l|l|l|}
\hline Process & $\mathrm{S}$ & $\mathrm{L}$ & $\mathrm{J}($ No $0 \leftrightarrow 0)$ & Parity \\
\hline \hline $\begin{array}{l}\text { Forced electric } \\
\text { dipole }\end{array}$ & $\Delta \mathrm{S}=0$ & $\Delta \mathrm{L} \leq 6$ & $\begin{array}{l}\Delta \mathrm{J} \leq 6, \text { if } \\
\Delta \mathrm{J}=2,4,6\left(\mathrm{~J} \text { or } \mathrm{J}^{\prime}=0\right)\end{array}$ & opposite \\
\hline Magnetic dipole & $\Delta \mathrm{S}=0$ & $\Delta \mathrm{L}=0$ & $\begin{array}{l}\Delta \mathrm{J}=0, \pm 1 \\
(0-0 \text { forbidden })\end{array}$ & same \\
\hline $\begin{array}{l}\text { Pseudo electric } \\
\text { quadrupole }\end{array}$ & $\Delta \mathrm{S}=0$ & $\Delta \mathrm{L}=0, \pm 1, \pm 2$ & $\Delta \mathrm{J}= \pm 1, \pm 2$ & opposite \\
\hline
\end{tabular}

\subsubsection{Photoluminescence of $\mathrm{Eu}^{2+}$ in fluoroperovskites}

Europium has an atomic number of 63 and its electron configuration is $6 \mathrm{~s}^{2} 4 \mathrm{f}^{7}$, which means the $f$ shell is only half filled and the electrons in the ground state have all the same 
spin. The optical absorption of Europium depends on the host in the crystal. The ground state of Europium ${ }^{2+}$ with the electron configuration $[X e] 4 f^{7}$ is ${ }^{8} \mathrm{~S}_{7 / 2}$ and this state is lifted into two excited states $4 f^{6} 5 d\left(T_{2 g}\right)$ and $4 f^{6} 5 d\left(E_{g}\right)$ due to the crystal field. The $4 f^{6} 5 d$ $\left(T_{2 g}\right)$ level is significantly lower than the $4 f^{7}\left({ }^{6} P_{7 / 2}\right)$, which leads to a broad d-f electron absorption and emission (several $1000 \mathrm{~cm}^{-1}$ ), which was reported for $\mathrm{Eu}^{2+}$ in $\mathrm{KMgF}_{3}$ and $\mathrm{RbMgF}_{3}$ by J. Garcia et al. [59].

The detection of only one broad band leads to the suggestion that the splitting of the crystal field, $10 \mathrm{Dq}$, of the compound is small. But since the splitting still exists, the $4 f^{6} 5 d\left(E_{g}\right)$ level is above the $4 f^{7}\left({ }^{6} P_{7 / 2}\right)$. This is proved by a sharp line emission from the $4 \mathrm{f}-4 \mathrm{f}$ transition. The $4 \mathrm{f}-4 \mathrm{f}$ transition is spin and parity forbidden in the electric dipole approximation. They can occur via state mixing, magnetic dipole, electric quadrupole, etc.. They take place at a lower rate and therefore these transitions have a longer lifetime than the $5 \mathrm{~d}-4 \mathrm{f}$ transition, which are allowed transitions. The $5 \mathrm{~d}-4 \mathrm{f}$ transition is allowed, and hence has a shorter lifetime. The coordination number of the $\mathrm{Eu}^{2+}$ ion in the compounds $\mathrm{KMgF}_{3}$ and $\mathrm{RbMgF}_{3}$, for example, is 12 as it is found on the $\mathrm{Rb}^{+}$and $\mathrm{K}^{+}$site because it is too large for the $\mathrm{Mg}^{2+}$ - ion [59]. Fig. 3.3 shows the configuration coordinate diagram for $\mathrm{RbMgF}_{3}: \mathrm{Eu}^{2+}$ and the splitting of the different levels by the crystal field. 


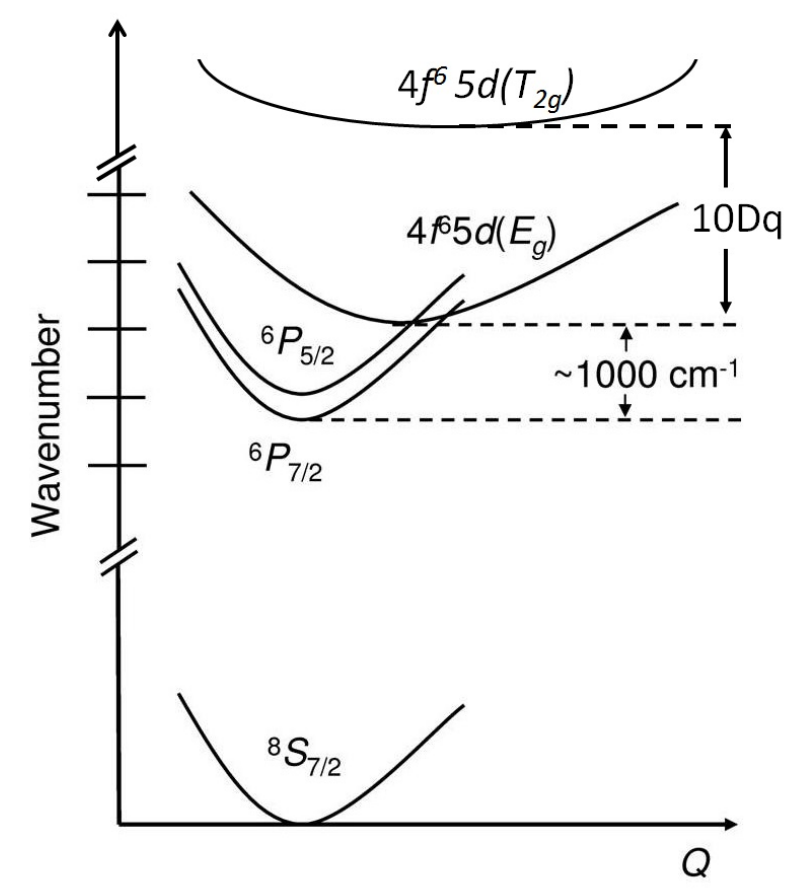

Figure 3.3: Configuration coordinate diagram of $\mathrm{Eu}^{2+}$ in $\mathrm{RbMgF}_{3}$ [4.

\subsubsection{Photoluminescence of $\mathrm{Mn}^{2+}$ in fluoroperovskites}

Manganese is on the $25^{\text {th }}$ place in the periodic table of elements and has an electronic configuration of $4 \mathrm{~s}^{2} 3 \mathrm{~d}^{5}$, which means the $d$ shell is only half filled and the electrons in the ground state form the spin sextet ${ }^{6} \mathrm{~S}$. The excited $d$ states have lower spin multiplicity, which means the corresponding transitions are spin and parity forbidden in the electric dipole approximation, but maybe partly allowed due to mixing of electron states and phonon modes with opposite symmetries as explained in the previous section.

A calculation of the energy levels for this electron configuration has been made by Tanabe and Sugano for the $\mathrm{Mn}^{2+}$ in octahedral coordination $\left(O_{h}\right.$ symmetry). This means in an octahedral complex 6 ligands surround a metal centre with a single pair on each axis. In this calculation, it has also been considered that the $d$ electron states (quartet states: ${ }^{4} \mathrm{~F},{ }^{4} \mathrm{D},{ }^{4} \mathrm{P},{ }^{4} \mathrm{G}$ ) are split by the crystal field when excited. This means, several optical 
transitions can be seen. These four quartet states can be seen on the left hand site of the Tanabe-Sugano diagrams. On the right hand site in the diagram, the different levels can be seen for a crystal field $10 D q \neq 0(10 \mathrm{Dq}$ is the splitting energy of the crystal field and is positive for octahedral coordination and negative for tetrahedral and cubic coordination) and the ground state as the $\mathrm{x}$-axis. The right axis in the Tanabe-Sugano diagram shows the symmetry properties, and the letters in the brackets lead to the energy level outside the crystal field. The superscripts are calculated by $2 S+1$ where $S$ is the total spin number of the crystal and the capital letters in the states $\left({ }^{6} \mathrm{~S},{ }^{4} \mathrm{~F},{ }^{4} \mathrm{D},{ }^{4} \mathrm{P},{ }^{4} \mathrm{G}\right)$ are the total orbital angular momentum $L$. The levels have a degeneracy of $2 L+1$, which can be lifted by the crystal field. In Fig. 3.4 the Tanabe-Sugano diagram of $\mathrm{Mn}^{2+}$ is shown [3, 57].

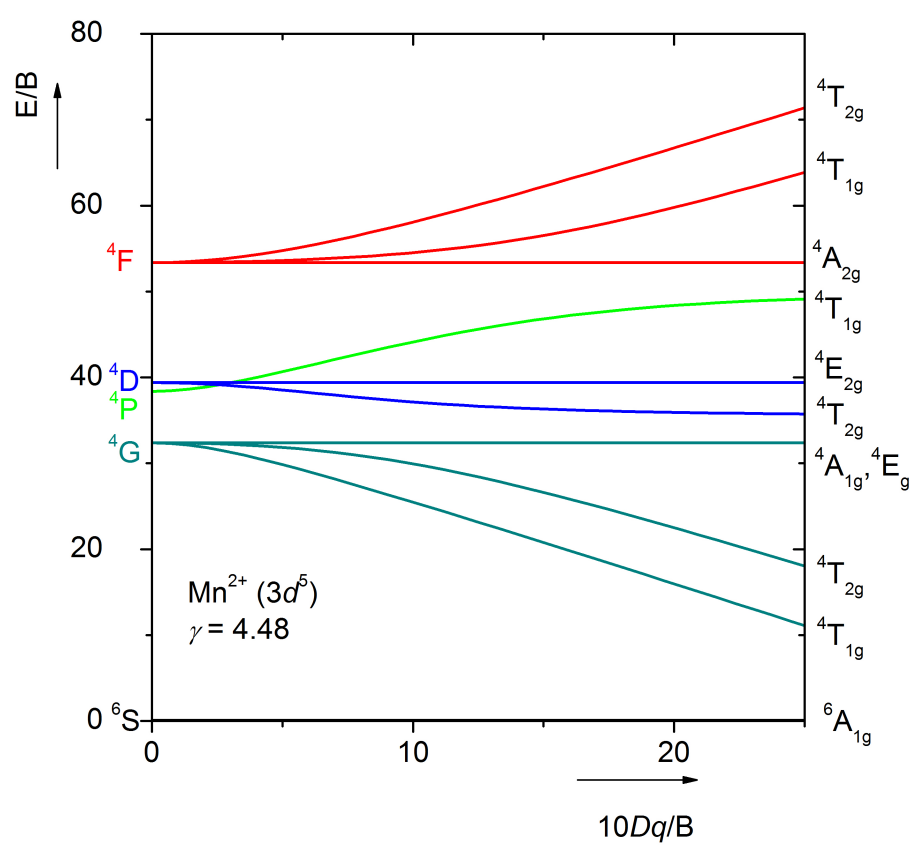

Figure 3.4: Energy levels of $\mathrm{Mn}^{2+}$ in a crystal field $10 \mathrm{Dq}[3]$. 


\subsubsection{Judd-Ofelt theory}

The Judd-Ofelt theory presents information on the local environment characteristics around a trivalent rare-earth ion and describes the transition intensities of these in a solid [20, 60]. The absorption spectra of the rare earth ions will be normally employed for calculation of the Judd-Ofelt parameters, but for $\mathrm{Eu}^{3+}$, which mainly will be investigated in this thesis, the corrected PL emission spectra can be employed. The reason for this is the nature of the $\mathrm{Eu}^{3+}$ transitions. The ${ }^{5} \mathrm{D}_{0} \rightarrow{ }^{7} \mathrm{~F}_{1}$ transition is a magnetic dipole transition where the oscillator strength is not influenced by the crystalline environment. This means, this transition can be utilised to compare the different spectra and calculate the Judd-Ofelt parameters from it [22]. This transition rate can be calculated with [61, 62, 63],

$$
A_{0-1}=\frac{64 \pi^{2} v^{3}}{3 h(2 J+1)} n^{3} S_{m d}=0.31 \times 10^{-11} n^{3} v_{0-1}^{3}
$$

with $\mathrm{h}$ as the Planck's constant, $v_{0-1}$ as the transition energy in $\mathrm{cm}^{-1}, n$ the effective refractive index with $n=1.364$ for $\mathrm{NaMgF}_{3}$ and $n=1.419$ for $\mathrm{RbMgF}_{3}$ and $\mathrm{S}_{m d}$ the magnetic line strength which is independent of the host. The other transitions are electric dipoles where the oscillator strength depends on the crystal field. These transition rates can be estimated from [63, 64],

$$
A_{0-J}=A_{0-1} \frac{I_{0-J} v_{0-1}}{I_{0-1} v_{0-J}}
$$

with $v_{0-J}$ as the relative transition energies in $\mathrm{cm}^{-1}$ and $\mathrm{I}_{0-J}$ the integrated emission intensities of the corrected PL spectra. Therefore, for $\mathrm{Eu}^{3+}$ the Judd-Ofelt parameters $\Omega_{2}$ and $\Omega_{4}$ can be calculated from the $0-\mathrm{J}$ transition rates [10, 22, 65, 66],

$$
A_{0-J}=\frac{100 \times 64 \pi^{4} v^{3} e^{2}}{3 h \times 4 \pi \epsilon_{0}} \frac{1}{2 J+1} \chi \Omega_{J}\left\langle{ }^{5} D_{0}\left\|U^{(J)}\right\|{ }^{7} F_{J}\right\rangle^{2}
$$

where $v$ is the transition energy in $\mathrm{cm}^{-1}, \epsilon_{0}$ the permittivity of free space, h the Planck's constant, e the electron charge and $\chi$ can be calculated by,

$$
\chi=n\left(\left(n^{2}+2\right) / 3\right)^{2}
$$


The reduced squared matrix elements are given by $\left\langle{ }^{5} D_{0} \| U^{(J)} \mid{ }^{7} F_{J}\right\rangle^{2}$, which were calculated for $\mathrm{LaF}_{3}$ but they can be employed for different systems because they are nearly independent of the crystalline environment [10, 20, 22, 66].

$\left\langle{ }^{5} D_{0}\left\|U^{(2)}\right\|{ }^{7} F_{2}\right\rangle^{2}=0.0032$

$\left\langle\left.{ }^{5} D_{0}\left\|U^{(4)}\right\|\right|^{7} F_{4}\right\rangle^{2}=0.0023$.

$\Omega_{2}$ and $\Omega_{4}$ are connected with short and long range effects, respectively. $\Omega_{2}$ should increase with a decreasing site symmetry, an increasing coordination number and a decreasing bond length [22].

\subsubsection{Photoluminescence lifetime measurements}

The average PL lifetime can be calculated from the PL decay curve, which can be fitted with multiple exponential components. The reason for fitting the PL with multi exponential fits is that the lifetime consists of a distribution of lifetimes and and they can be averaged to one lifetime. The ideal case would be a single exponential fit, which shows that there exist only one lifetime of the ions. The average lifetime was then calculated using [67],

$$
\tau_{\text {ave }}=\left[\int t I(t) d t / \int I(t) d t\right] .
$$

where the time $t$ is multiplied with the individual t-dependent term, $I(t)$, divided by the total t-dependence term. The radiative lifetime can be calculated as [68],

$$
\tau_{\text {rad }}=\frac{1}{\sum_{J} A_{0-J}}
$$

where $\mathrm{A}_{0-J}$ are the $0-\mathrm{J}$ transitions rates calculated with the Judd-Ofelt theory in the previous section.

The quantum efficiency is defined as the probability of a photon being emitted to the probability of a photon being absorbed. This can be calculated from the ratio of the 
measured average lifetime divided by the radiative lifetime. Hence, the quantum efficiency for the $\mathrm{Eu}^{3+}$ ion can be written as [69],

$$
\eta=\frac{\tau_{\text {ave }}}{\tau_{\text {rad }}}
$$

\subsection{Thermoluminescence}

As already mentioned in the introduction of this thesis, thermoluminescence (TL) is a phenomena where irradiation induces carriers which will be trapped until heating the sample (read-out). This leads to recombination of the trapped charges and consequent emission of light, with the generation of a "glow curve". TL provides additional information of the trapping centres via the fitting of the glow curve and calculating the activation energy of the traps by first order kinetics deconvolution, as performed in this thesis.

The material is in equilibrium before applying ionised radiation. In the crystal, there exist trapping states for the electrons with concentration $N$ and holes with concentration $M$. Incoming X-rays create mobile holes in the valence band with a concentration of $m_{v}\left(\mathrm{~cm}^{-3}\right)$ and electrons in the conduction band with a concentration of $n_{c}\left(\mathrm{~cm}^{-3}\right)$. Electrons get trapped with the probability coefficient $A_{n}$. After a thermal stimulation of these trapped electrons, some electrons might fall directly back to the trapping states and some electrons will recombine with the trapped holes with the recombination probability $A_{m}$. To get the information about the total absorbed dose by the material, thermally stimulation will be applied. By measuring the intensity of the emitted light, the received dose can be estimated. The release of energy can be via an electron-hole recombination which is illustrated in Fig. 3.5. 


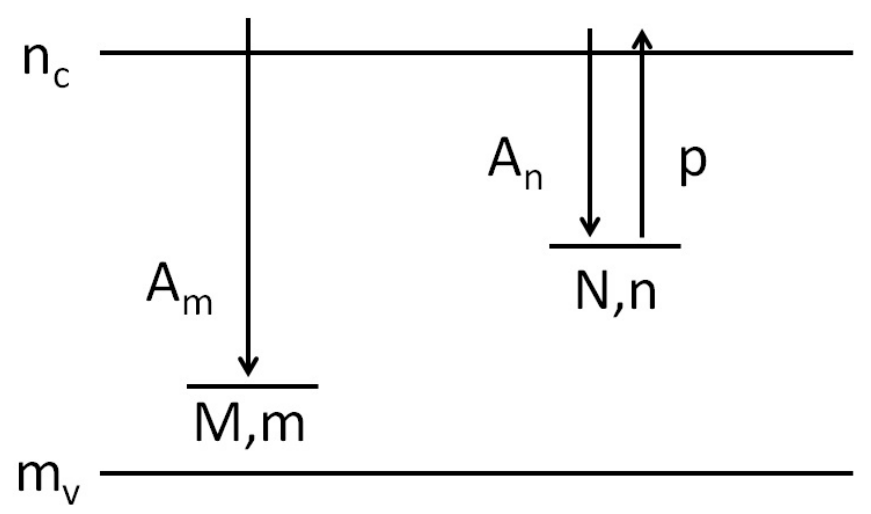

Figure 3.5: One-trap/ one- centre model for the TL process [5].

Furthermore, the probability of releasing a trapped charge and overcome the barrier E is presented by the Arrhenius equation:

$$
p(T)=s(T) e^{-\frac{E}{k T}}
$$

with k the Boltzmann's constant and $\mathrm{s}(\mathrm{T})$ a frequency factor which is related to the local lattice vibrational frequency and the entropy change cause by charge release. With increasing temperature, the particle can overcome the barrier $\mathrm{E}$ and the particle can move into $\mathrm{n}_{c}$. If this recombination is radiative, it is called thermoluminescence [5]. p varies over time when increasing the temperature during a TL read-out with a constant heating rate $\beta=$ $\mathrm{dT} / \mathrm{dt}$. The detected TL is the so-called glow curve, which is a plot of the luminescence intensity over the temperature. A one trap model with finite re-trapping probability and one recombination centre can be modelled by the following rate equations,

$$
\begin{gathered}
\frac{d m}{d t}=-A_{m} m n_{c} \\
\frac{d n}{d t}=-n s e^{-\frac{E}{k T}}+A_{n}(N-n) n_{c} \\
\frac{d n_{c}}{d t}=\frac{d m}{d t}-\frac{d n}{d t}
\end{gathered}
$$

with the assumption $\frac{d n_{c}}{d t} \ll \frac{d m}{d t}, \frac{d n}{d t}$ and,

$$
I_{T L}=-\frac{d m}{d t}
$$


$\mathrm{dn} / \mathrm{dt}$ can be written as,

$$
\begin{gathered}
\frac{d n}{d t}=-n s e^{-\frac{E}{k T}}+A_{n}(N-n) n_{c}=-A_{m} m n_{c} \\
n_{c}\left(A_{m} m+A_{n}(N-n)\right)=n s e^{-\frac{E}{k T}} \\
n_{c}=\frac{n s e^{-\frac{E}{k T}}}{A_{m} m+A_{n}(N-n)}
\end{gathered}
$$

Eliminating $\mathrm{n}_{c}$ leads to,

$$
\frac{d n}{d t}=-n s e^{-\frac{E}{k T}}\left(1-\frac{A_{n}(N-n)}{A_{m} m+A_{n}(N-n)}\right)
$$

In this thesis, the activation energies and TL peaks were calculated via first order kinetics deconvolution which is described by the Randall and Wilkins expression, and assumes that re-trapping is negligible, $\mathrm{mA}_{m} \gg(\mathrm{N}-\mathrm{n}) \mathrm{A}_{n}$ then equation 3.17 becomes,

$$
\frac{d n}{d t}=-n s e^{-\frac{E}{k T}}
$$

and using $\mathrm{T}(\mathrm{t})=\mathrm{T}_{0}+\beta \mathrm{t}$, dn/dt can be integrated to,

$$
I_{T L}=n_{o} s \exp \left(-\frac{E}{k T}\right) \exp \left[-\frac{s}{\beta} \int_{T_{0}}^{T} \exp \left(-\frac{E}{k T^{\prime}}\right) \mathrm{d} T^{\prime}\right]
$$

where $\mathrm{n}_{o}$ is the concentration at $\mathrm{T}_{o}, \beta$ is the heating rate and ' $\mathrm{T}^{\prime}$ is a variable representing the temperature [5]. The first term in equation 3.19 represents what is happening during the stimulation and the second term describes the energy loss.

\subsection{Radioluminescence}

Radioluminescence (RL) has recently attracted considerable interest, because it can be employed to determine the real-time radiation dose rate for radiation protection, nondestructive testing, and in medicine for monitoring radiation dose during radiotherapy or for dose verification and validation [10, 11, 12, 70, 71. Fig. 3.6 shows a symbolic RL 
recombination process and the process involves an electron - hole generation and recombination at a luminescent ion. During this process, the X-rays get absorbed and this causes the creation of electrons and holes in the conduction and valence band. These electrons and holes are mobile and can recombine (on a luminescent ion $\left(\mathrm{A}^{*}\right)$. The light emitted by radioluminescence after absorption of radiation is usually detected with photomultiplier tubes (PMT) or charge-couple device (CCD) detectors. Radioluminescent materials are of interest, which emit light in the region from $200 \mathrm{~nm}$ till $700 \mathrm{~nm}$ (visible light), because the PMT detects from $\sim 350 \mathrm{~nm}-700 \mathrm{~nm}$ and the CCD from $\sim 400 \mathrm{~nm}-800 \mathrm{~nm}$ [72].

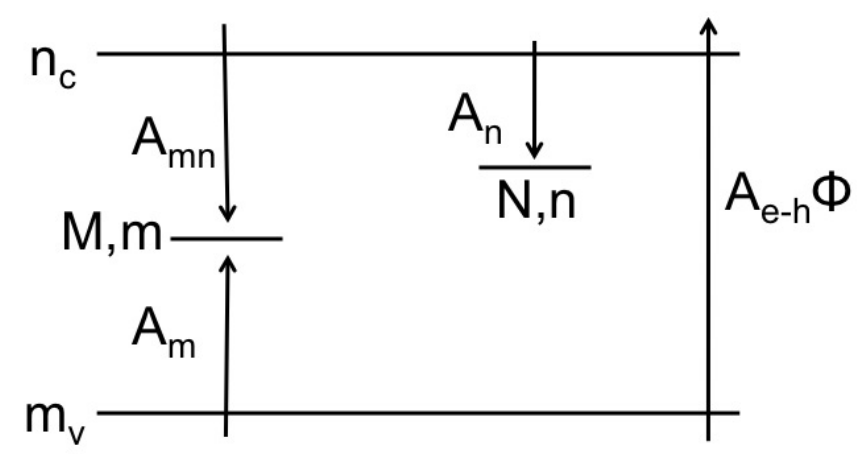

Figure 3.6: Model of the one trap, one recombination centre for RL.

In Fig. 3.6, $N$ is the total concentration of traps and $M$ is the total concentration of hole traps. $m_{v}$ and $n_{c}$ are the concentration of holes and electrons in the valence and conduction band, respectively. $A_{e-h}$ is the ionisation probability and $\Phi$ the radiation flux. $A_{n}$ is the probability coefficient where electrons get trapped and $A_{m}$ the recombination probability where some electrons will recombine with the trapped holes. Therefore, the following rate equations were estimated [73],

$$
\begin{gathered}
\frac{d n}{d t}=A_{n}(N-n) n_{c} \\
\frac{d n_{c}}{d t}=A_{e-h} \Phi-A_{m n} m n_{c}-\frac{d n}{d t} \\
\frac{d m}{d t}=-A_{m n} m n_{c}+A_{m}(M-m) m_{v} \\
\frac{d n_{v}}{d t}=A_{e-h} \Phi-A_{m}(M-n) m_{v}
\end{gathered}
$$




$$
I_{R L} \sim \frac{d m}{d t}
$$

\subsection{Optically Stimulated Luminescence}

The process of stimulated luminescence in general means that through radiation the sample absorbs energy, which causes ionisation of valence electrons, the freed electrons and holes drift through the crystal and get trapped in defects. Energy will be absorbed via the stimulation of the sample with i.e. UV, visible or IR light, which causes recombination of trapped carriers. During a radiative recombination luminescence can be measured [6].

The material before the radiation is in equilibrium and becomes metastable during the radiation until the stimulation. After the stimulation the state of the system is in equilibrium again [6]. In OSL the intensity of the emitted luminescence is correlated to the rate of the electrons and holes which recombine. This rate is a function of the concentration of the charged particles. Usually a luminescence versus time curve is plotted and the integral of this graph is connected to the trapped particles, which is proportional to the dose of absorbed radiation [6]. For a fixed energy with a continuous illumination the probability to stimulate the trapped carriers is dependent on the energy and the illumination intensity $\Phi$, and is not time-independent. This type of OSL is called continuous wave OSL, CW-OSL, and the shape of the OSL intensity versus time graph is a decay curve. One way to simplify the OSL process is to assume that the system has one type of electron trap and one type of hole trap (see Fig. 3.7), which is also called the one-trap/ one-centre model and equation 3.25 shows the charge neutrality for this system [74, 75].

$$
n_{c}+n=m_{v}+m
$$

In the above equation $3.25, n_{c}$ is the concentration of the electrons in the conduction band, $n$ the concentrations of the electrons in the traps, $m_{v}$ the concentration of the holes in the valence band and $m$ the concentration of the recombination centres. 


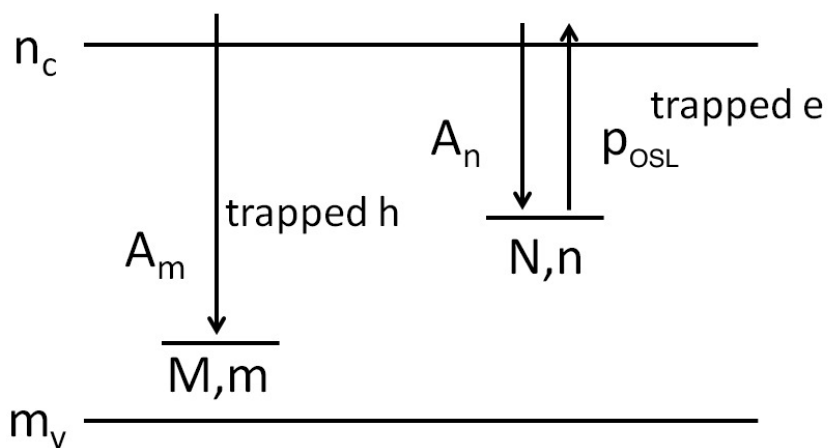

Figure 3.7: One-trap/ one-centre OSL model [6].

The material is in equilibrium before applying ionised radiation. $N$ is the total concentration of electron traps and $M$ the total concentration of hole traps. Incoming X-rays create mobile holes in the valence band with a concentration of $m_{v}\left(\mathrm{~cm}^{-3}\right)$ and electrons in the conduction band with a concentration of $n_{c}\left(\mathrm{~cm}^{-3}\right)$. Electrons get trapped with the probability coefficient $A_{n}$. After an optical stimulation of these trapped electrons, some electrons might fall directly back to the trapping states and $A_{m}$ is the recombination probability where some electrons will recombine with the trapped holes. The derivative of equation with respect to the time $t$ and for a one trap model with finite re-trapping probability and one recombination centre,

$$
\begin{aligned}
\frac{d m}{d t} & =-A_{m} m n_{c} \\
\frac{d n}{d t} & =-n p_{O S L} \\
\frac{d n_{c}}{d t} & =\frac{d m}{d t}-\frac{d n}{d t}
\end{aligned}
$$

with the assumption of slow re-trapping $\frac{d n_{c}}{d t} \ll \frac{d m}{d t}, \frac{d n}{d t}$ and,

$$
I_{O S L}=-\frac{d m}{d t}
$$

dn/dt can be written as,

$$
\frac{d n}{d t}=-n p_{O S L}+A_{n}(N-n) n_{c}=-A_{m} m n_{c}
$$




$$
\begin{gathered}
n_{c}\left(A_{m} m+A_{n}(N-n)\right)=n p_{O S L} \\
n_{c}=\frac{n p_{O S L}}{A_{m} m+A_{n}(N-n)}
\end{gathered}
$$

Eliminating $\mathrm{n}_{c}$ leads to,

$$
\frac{d n}{d t}=-\frac{n p_{O S L} A_{m} m}{A_{m} m+A_{n}(N-n)}
$$

The one-trap/ one-centre model is a good way to also describe non-exponential OSL curves [6, 31, 74, 75]. Examples of traps include:

- An F-entre is a vacancy with a trapped electron and in the case of $\mathrm{Al}_{2} \mathrm{O}_{3}$ the $\mathrm{F}^{+}$centres are recombination centres.

- $\mathrm{V}_{k}$-centre or a $\mathrm{X}_{2}^{-}$-centre means a hole trapped between two halide ions [3]. 


\section{Experimental techniques}

\subsection{Sample preparation}

In this thesis different types of samples are produced, which are described in the following paragraphs. The samples are utilised for 1D dosimeters and the starting compounds are usually polycrystalline bulk materials or nanoparticles, which are further fabricated as thin films or pressed as pellets, respectively. All samples are prepared in glove boxes under an inert atmosphere to protect the materials and ensure that the operating person does not inhale hazardous powder.

\subsubsection{Polycrystalline samples}

The polycrystalline bulk samples were prepared at Victoria University of Wellington. The compounds are produced by mixing the starting materials (e.g. $\mathrm{RbF}, \mathrm{NaF}, \mathrm{MgF}_{2}$, and either $\mathrm{MnF}_{2}$ or $\mathrm{EuF}_{2}$ ) in the stoichiometric ratio in a glassy carbon crucible and are sealed with a glassy carbon lid. The mixing is performed in a sealed glove box under nitrogen ("dry" or "oxygen free") atmosphere. After that, the crucible with the powder was transported to the furnace, which is connected to another glove box. The samples are then heated with a furnace consisting of a quartz tube where a central zone was heated by a radio frequency heating utilising a graphite susceptor. The samples were heated slightly above the melting temperature, and then slowly cooled down below the melting point. 
With a computer program, a defined heating pattern was written for the furnace. During this heating a constant argon flow was applied. The different sample concentrations that were studied and reported in this thesis were chosen because these concentrations were used in the fibre optic dosimeter in the Wellington Hospital.

\subsubsection{Nanoparticles}

The motivation of preparing nanoparticles is to get transparent plates of an arbitrary size and shape for different types and application of dosimeters. The fluoroperovskite $\mathrm{RbMgF}_{3}$ and $\mathrm{NaMgF}_{3}$ nanoparticles doped with $1 \%, 2 \%$ or $5 \% \mathrm{Mn}$ or Eu were produced using a reverse micro-emulsion method [76]. The precursors were high purity $\mathrm{RbNO}_{3} \times 6 \mathrm{H}_{2} \mathrm{O}$, $\mathrm{NaNO}_{3} \mathrm{x} 6 \mathrm{H}_{2} \mathrm{O}, \mathrm{MgNO}_{3} \mathrm{x} 6 \mathrm{H}_{2} \mathrm{O}, \mathrm{Mn}\left(\mathrm{NO}_{3}\right)_{3}, \mathrm{Eu}\left(\mathrm{NO}_{3}\right)_{3}$, Cetyl trimethylammonium bromide $(\mathrm{CTAB})\left(\left(\mathrm{C}_{16} \mathrm{H}_{33}\right) \mathrm{N}\left(\mathrm{CH}_{3}\right)_{3} \mathrm{Br}\right)$ and $\mathrm{NH}_{4} \mathrm{~F} .10 \mathrm{~g}$ of CTAB and $50 \mathrm{ml}$ of 2-octanol were placed in a flask and stirred for about 1 hour. The nanoparticles were produced by first dissolving (370.4mg, $10 \mathrm{mmol}$ ) $\mathrm{NH}_{4} \mathrm{~F}$ into $4.6 \mathrm{ml}$ of milliQ water. This was then added to the solution containing $10 \mathrm{~g}$ of CTAB and $50 \mathrm{ml}$ of 2-octanol and stirred for 30 minutes (flask A). $3 \mathrm{mmol}$ of $\mathrm{RbNO}_{3}$ or $\mathrm{NaNO}_{3}, 2.5 \mathrm{mmol}$ of $\mathrm{MgNO}_{3}$ and $\mathrm{x}$ mmol of $\mathrm{Mn}\left(\mathrm{NO}_{3}\right)_{3}$ or $\mathrm{Eu}\left(\mathrm{NO}_{3}\right)_{3}$, were dissolved in $4.6 \mathrm{ml}$ of milliQ water. This was then added to a solution containing $10 \mathrm{~g}$ of CTAB and $50 \mathrm{ml}$ of 2-octanol and also stirred for 30 minutes (flask B). The contents of flask B were slowly added to flask A while stirring for about 10 minutes after which the nanoparticles precipitated. The solution was diluted with ethanol and centrifuged at 4000 rpm for 3 to 5 minutes to isolate and purify the nanoparticles. This process was repeated 5 times. Fluoride nanoparticles were manufactured with a different method which will be explained in the particular chapter. 


\subsection{X-ray diffraction}

After the preparation of the samples (polycrystalline bulk materials, nanoparticles or the thin sheets, e.g.). The samples were characterised with X-ray diffraction (XRD) to ensure the purity and to examine if any process affects the purity of the compound. In most of the XRD measurements, the samples were ground until they form a fine powder and the diffraction pattern was recorded for the powder. The powder is placed either on aluminium powder sample holders (at Callaghan Innovation), or on silicon powder sample holders (at VUW). The X-ray diffraction patterns of the samples were performed with an X-ray generator (Model PW 1729, Philips, Holland) with crystal monochromatic Co $\mathrm{K}_{\alpha}(\lambda=1.79 \AA)$ radiation. At Victoria University, the XRD patterns were collected with $\mathrm{Cu} \mathrm{K}_{\alpha}$ X-rays $(1.54 \AA)$ radiation. The patterns were then compared with reference patterns from the International Centre for Diffraction Data database. Fig. 4.1 shows the XRD patterns of $\operatorname{RbMgF}_{3}(\mathrm{a})$ bulks, $\mathrm{NaMgF}_{3}(\mathrm{~b})$ bulks, $\mathrm{RbMgF}_{3}(\mathrm{c})$ nanoparticles and $\mathrm{NaMgF}_{3}(\mathrm{~d})$ nanoparticles. XRD pattern for the $\mathrm{LaF}_{3}$ and $\mathrm{BaMgF}_{4}$ are already published elsewhere [19, 22, 77]. The JCPDS reference pattern is 01-070-8139 for $\mathrm{NaMgF}_{3}$ and 01-076-0179 for $\mathrm{RbMgF}_{3}$ with an orthorhombic $\left(\mathrm{P}_{b n m}\right)$ and hexagonal $\left(\mathrm{P}_{6_{3} n m}\right)$ phase for the bulk materials, respectively. For the nanoparticles, the JCPDS reference pattern is 04-012-1614 for $\mathrm{NaMgF}_{3}$ and $04-008-2832$ for $\mathrm{RbMgF}_{3}$ with an orthorhombic $\left(\mathrm{P}_{\text {bnm }}\right)$ and hexagonal $\left(\mathrm{P}_{6_{3} n m}\right)$ phase, respectively. 


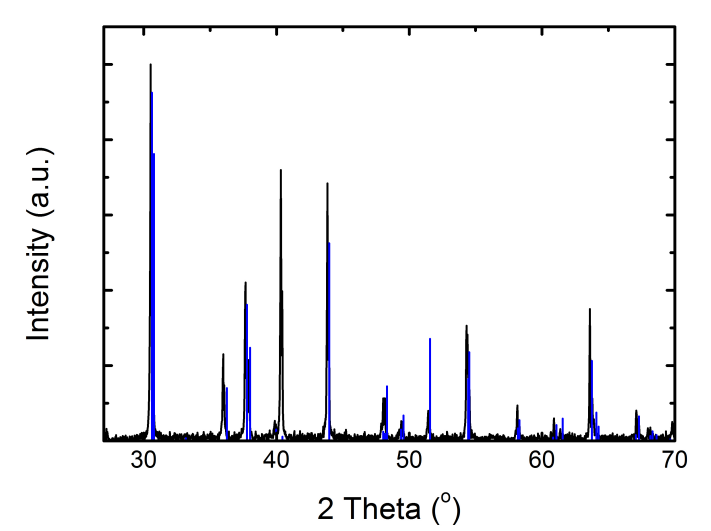

(a)

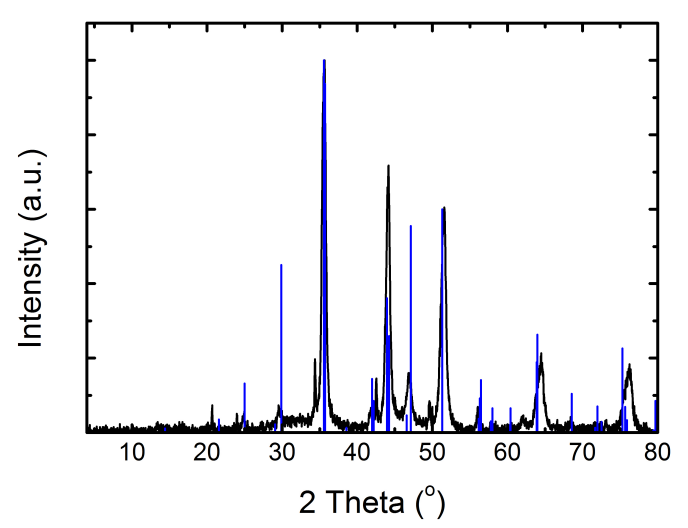

(c)

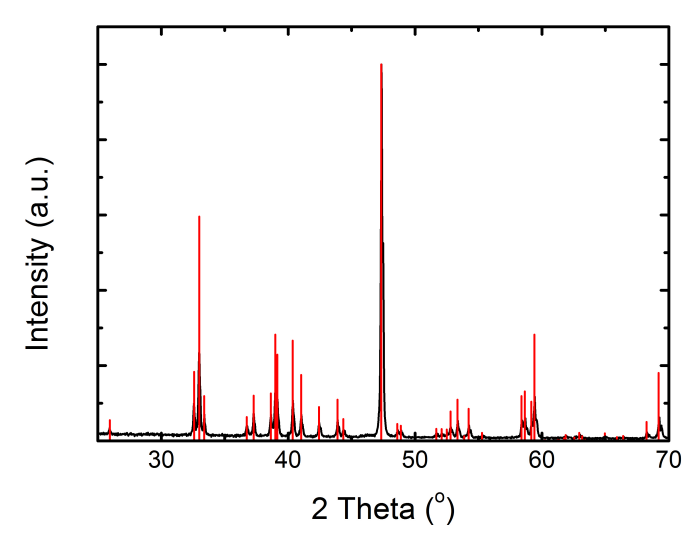

(b)

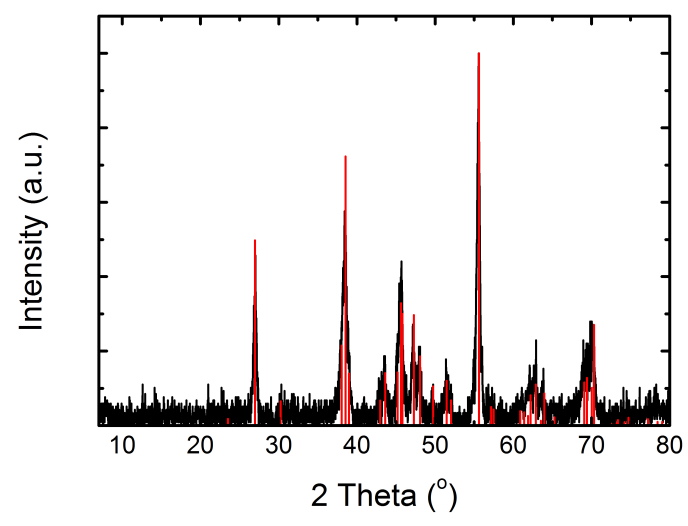

(d)

Figure 4.1: XRD diffractogram of $\mathrm{RbMgF}_{3}(\mathrm{a})$ and $\mathrm{NaMgF}_{3}(\mathrm{~b})$ bulks and the reference pattern for $\mathrm{RbMgF}_{3}$ (blue lines in (a)) and $\mathrm{NaMgF}_{3}$ (red lines in (b)).

XRD diffractogram of $\mathrm{RbMgF}_{3}(\mathrm{c})$ and $\mathrm{NaMgF}_{3}(\mathrm{~d})$ nanoparticles and the reference pattern for $\mathrm{RbMgF}_{3}$ (blue lines in (c)) and $\mathrm{NaMgF}_{3}$ (red lines in (d)).

The crystal size $l$ (grain size) of the nanoparticles was estimated with the Scherrer formula [78],

$$
\begin{array}{r}
l=\frac{K \lambda}{\beta \cos (\theta)}, \\
\text { with } \beta=\frac{1}{2}\left(2 \theta_{2}-2 \theta_{1}\right)
\end{array}
$$

where $\mathrm{K}$ is a constant which is close to unity (0.9) and related to the crystalline shape, 
which is assumed to be spherical, $\lambda$ is the X-ray wavelength and $\theta$ is the Bragg angle [78].

\subsection{Thermoluminescence measurements}

TL measurements were performed with a custom-built TL spectrometer. The schemata of the TL measurements are illustrated in Fig. 4.2. The samples were powdered, immersed in isopropanol and dispersed on two rectangular PT 100 resistor (sample holder) for controlled heating up to $350{ }^{\circ} \mathrm{C}$. The sample holder was $2 \mathrm{~mm}$ x $3 \mathrm{~mm}$ big and usually a $1 \mathrm{~mm}$ thick layer of the powder isopropanol mixture was placed on it. The sample holder was heated up to $350{ }^{\circ} \mathrm{C}$ outside of the light-tight cylinder to evaporate all of the isopropanol. For better heat transport in the sample, the powder is usual immersed with silicon oil, but this oil shows a signal in the TL, which overlaps with the signal of the samples. Therefore, isopropanol was utilised instead. This sample holder was placed in a light-tight cylinder, which has a connection to a fibre cable on the top and to the X-ray generator on the front. All measurements were performed in a lead box, which surrounds the X-rays generator. The emission was detected with an optical fibre that was connected to a photomultiplier with a photon-counting unit or a CCD (charged-couple device). The CCD was used for a high intensity TL signal, for example with the bulk materials. It was possible to observe which transition is having an impact on the TL signal with the CCD. The nanoparticles did not have an intense TL signal and therefore the PMT (photomultiplier tubes) was utilised to detect the TL glow curve. It was not possible to get any information about the origin of the TL signal because the PMT only detects the overall signal of the TL glow curve and does not detect an extra TL intensity versus wavelength spectra for each temperature step compared to the measurements with the CCD. After each TL measurement, a black body measurement was performed to subtract the background from the actual TL signal. The X-ray irradiations were carried out with a Philips X-ray generator operating at $40 \mathrm{kV}$ and $40 \mathrm{~mA}$, using a W-tube. The glow curves were measured with different heating rate $1 \mathrm{~K} / \mathrm{s}$ and at different doses. 


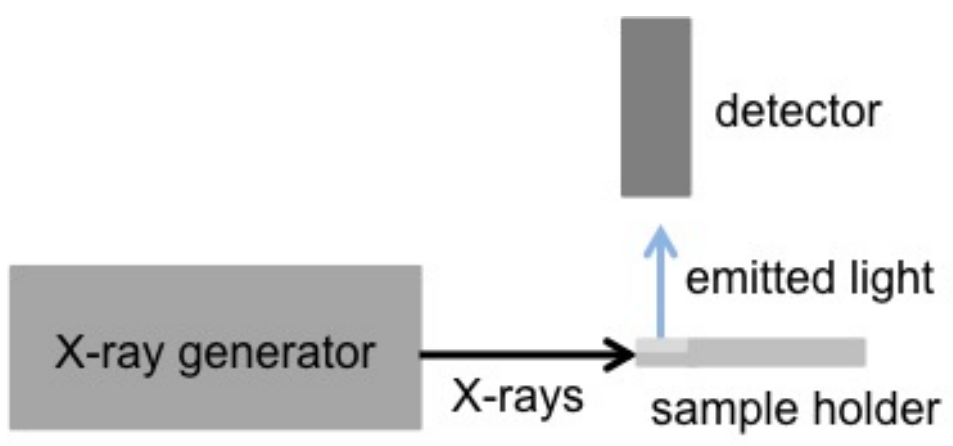

Figure 4.2: Schematic of the thermoluminescence measurements.

\subsection{Radioluminescence measurements}

The ${ }^{241} \mathrm{Am}$ RL measurements were performed with a custom-built system that was designed especially for these measurements. A schematic of this holder is illustrated in Fig. 4.3. It consists of two parts: the lower part holds the PMT and filters can be adjusted and an upper part, which holds the sample and the source. Between the upper and lower part a foil is attached, which keeps the sample in position. Right above the sample, the holder for the ${ }^{241} \mathrm{Am}$ source starts. The source can be adjusted to a different height depending on purpose of the measurements, which needs to be performed. The two parts of the holder are screwed to each other, and they are constructed of lead to protect the person who is measuring being exposed to harmful radiation. Additionally on the top of the upper holder, there is a lead screw to protect the operator from radiation, to easily remove or bring the source into the sample holder and to protect the PMT from being exposed to light from the environment during the measurement. This setup captures the whole emitted light of the sample during the exposure by the radiation source with the PMT. 


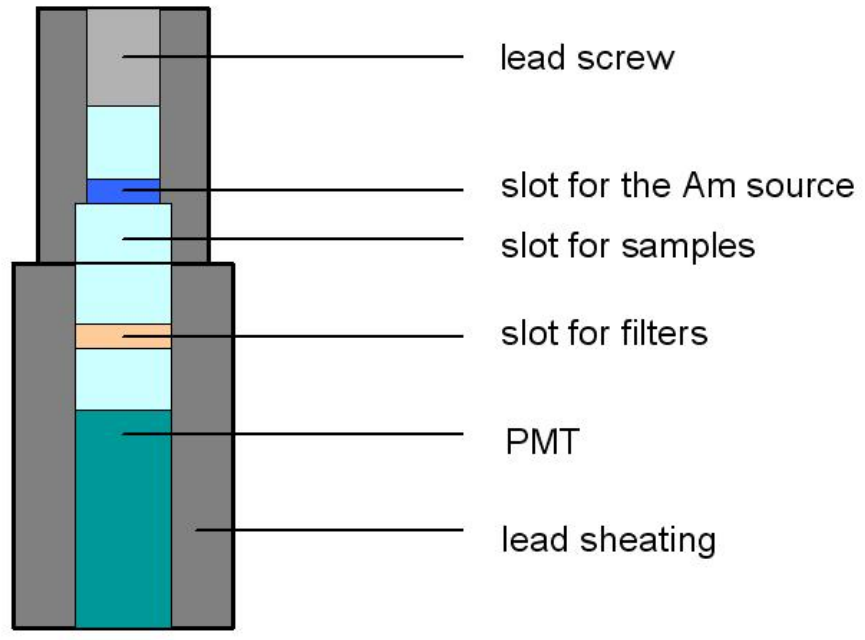

Figure 4.3: Schematic of the radioluminescence measurements with the ${ }^{241} \mathrm{Am}$-source.

Higher dose measurements were performed using a custom-built system attached to an $\mathrm{X}$-ray set using X-rays generated by a cobalt X-ray tube (40 kV and $40 \mathrm{~mA})$ with an Aluminium filter. The sample was located $\sim 3 \mathrm{~cm}$ from the exit port (similar to the TL measurements, explained in the previous section). The only difference is that the sample was placed on a holder with an $45^{\circ}$ angle. This system was also utilised to collect the RL spectra where an optical fibre was connected to an Ocean Optics USB 2000 or 4000 spectrometer that had a CCD detector array.

\subsection{Radiation facilities}

\section{${ }^{241} \mathrm{Am}$ radio nuclide}

The ${ }^{241} \mathrm{Am}$ source emits up to $0.4 \mathrm{mGy} / \mathrm{s}$ (at a distance of $0.1 \mathrm{~cm}$ ) of monochromatic $\gamma$-rays with an energy of about $60 \mathrm{keV}$ with an activity of $3500 \mathrm{MBq}$. Most of the measurements or irradiations of the sample to measure RL, OSL or conversion efficiency OSL are performed by placing the source directly on the sample. For some RL measurements, the distance between the sample and the source is varied with the sample holder described 
in the preceding paragraph. The dose rates on the sample vary with the distance from the source, which has to be taken into account when comparing the measurements done with a different setup.

\section{Philips PW 1720 X-ray tube with W-anode}

The X-ray beam was employed with a tube voltage of $40 \mathrm{kV}$ and a current of $40 \mathrm{~mA}$. The distance in this tube can be varied. It can be utilised either without a filter, with an $\mathrm{Al}$ filter or a quartz window.

\subsection{Photoluminescence, PL lifetime and temperature dependent PL measurements}

\section{Cary Eclipse fluorescence spectrometer}

Photoluminescence measurements performed at the Callaghan Innovation were carried out with the Varian Cary50 Eclipse. It operates with a pulsed Xe-lamp with a pulse of 2-3 $\mu \mathrm{s}$ and a repetition rate up to $80 \mathrm{~Hz}$. The schematic configuration of the Cary Eclipse is illustrated in Fig. 4.5 [7]. The wavelength range of the pulsed Xenon lamp is from 190 $\mathrm{nm}$ to $1100 \mathrm{~nm}$. The device offers a different range of slit widths both for the emission and excitation beam, for example 1.5, 2.5, 5, 10, $20 \mathrm{~nm}$ and a round slit of $10 \mathrm{~nm}$. For the excitation and emission detection, there are also built-in filters as well as a possibility to insert an external filter in the device. Spectra can be detected with the fluorescence or phosphorescence mode. The total decay time, gate time and delay between the pulse and start of the measurement can be selected with the phosphorescence mode. For fluorescence measurements, the signal is measured during the excitation pulse. Lifetime measurements can be performed with a time resolution down to $1 \mu \mathrm{s}$ [7]. 


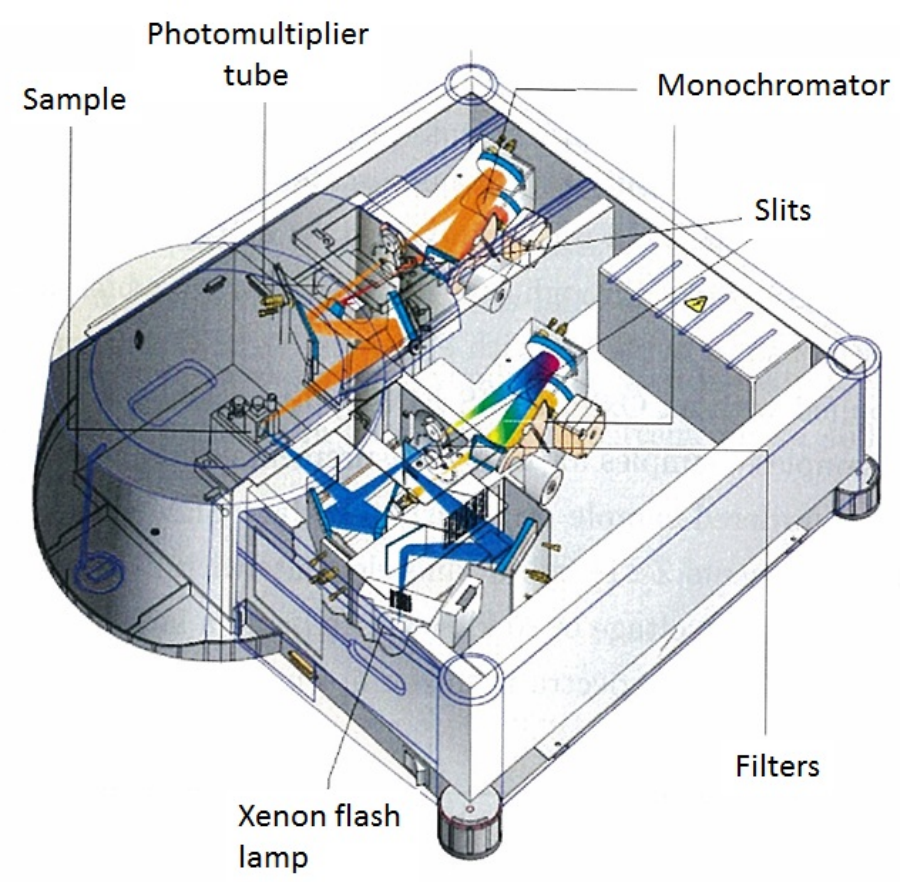

Figure 4.4: Schematic configuration of the Varian Cary Eclipse fluorescence spectrometer[7].

\section{Jobin-Yvon FluoroLog $\mathbb{B}$}

At Victoria University of Wellington, photoluminescence (PL), PL lifetime, temperature dependent PL and OSL measurements were performed using the Jobin-Yvon FluoroLog ${ }^{\circledR}$ spectrometer which operates over a wide wavelength range, from $250 \mathrm{~nm}$ until $1550 \mathrm{~nm}$, by employing a Xe short-arc lamp. This spectrometer was also utilised to perform lifetime measurements from a few $\mu$ s or even longer. The setup was slightly modified and three different LEDs were utilised as an excitation source (370nm, $465 \mathrm{~nm}, 498 \mathrm{~nm}$ ). This method is called multichannel scaling single-photon-counting spectroscopy (MCS). Low temperature PL and lifetime measurements were performed for temperatures down to 10 $\mathrm{K}$ with an RMC-22 closed cycle refrigerator [8]. 


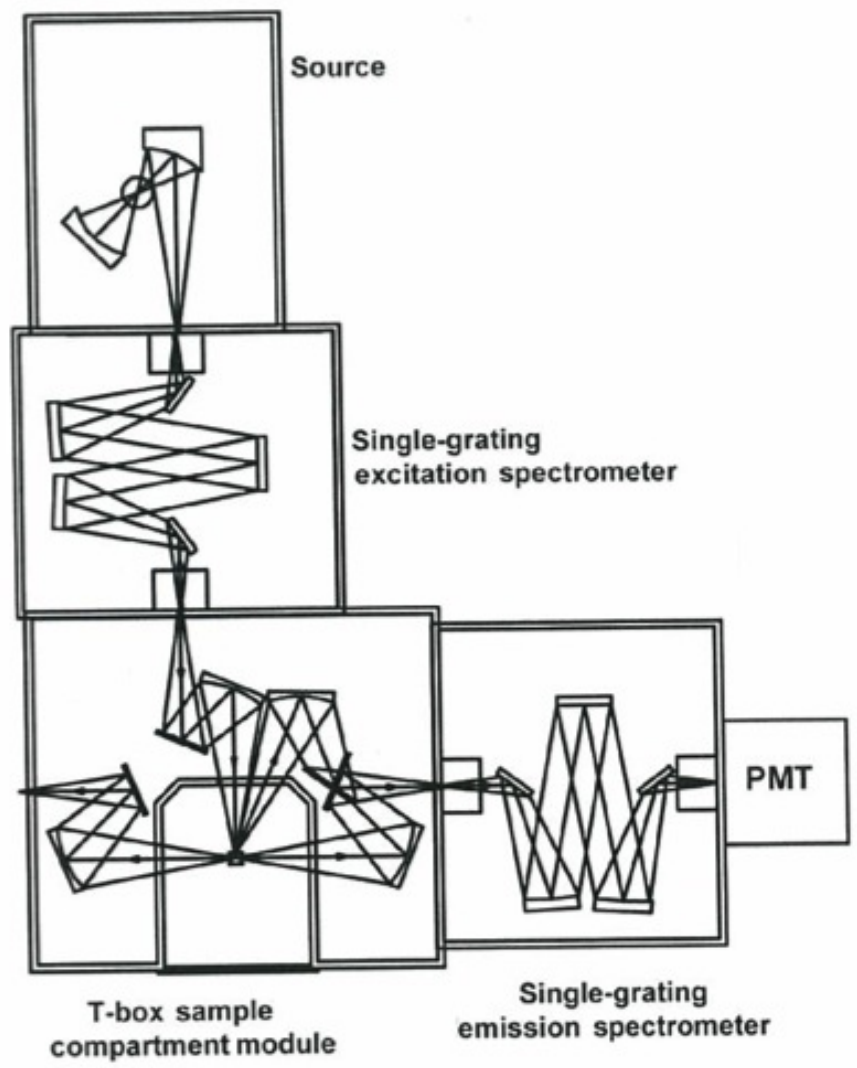

Figure 4.5: Schematic configuration of the Jobin-Yvon FluoroLog ${ }^{\circledR}$ spectrometer [8]. 


\section{Bulk polycrystalline $\mathrm{NaMgF}_{3}$}

In this chapter, the optical, radioluminescent and thermoluminescent properties of Eu and Mn doped $\mathrm{NaMgF}_{3}$ will be discussed. The samples were prepared as described in the experimental description given in section 4.1.1. $10 \mathrm{~g}$ of each sample was produced using high purity Sodium fluoride $(\mathrm{NaF})$, Magnesium fluoride $\left(\mathrm{MgF}_{2}\right)$, Europium(II) fluoride $\left(\mathrm{EuF}_{2}\right)$ or Manganese(II) fluoride $\left(\mathrm{MnF}_{2}\right) \cdot \mathrm{NaMgF}_{3}$ is also called neighborite, and the crystal structure is orthorhombic with the space group $P_{b n m}$ and a melting point at $\sim 1030$ ${ }^{o} \mathrm{C}$. The bandgap is about $6.05 \mathrm{eV}[79]$ and the $4 \mathrm{f}^{6} 5 \mathrm{~d}\left(\mathrm{E}_{g}\right)$ levels are above the ${ }^{6} \mathrm{P}_{J}$ levels with an energy difference of $\Delta \mathrm{E}=1.2 \mathrm{eV}$ which is around $9700 \mathrm{~cm}^{-1}$ [3, 9, 79, 80, 81]. The significance of the energy difference is the bigger the gap the lower the probability of $5 \mathrm{~d} 4 \mathrm{f}$ transition. The samples which will be discussed were doped with either $0.2 \%$ $\mathrm{Eu}, 0.5 \% \mathrm{Mn}$ and $1 \% \mathrm{Mn}$. The appearance of the Eu doped sample was slightly milky and transparent. The Mn doped samples were slightly greenish and transparent, but the $1 \%$ doped sample was a bit darker than the $0.5 \%$ doped sample. The influence the concentration of the Mn dopant has on the PL spectra, PL lifetime, TL and RL measurements will be demonstrated. 


\subsection{Photoluminescence and PL lifetime measurements}

\subsubsection{Eu doped polycrystalline $\mathrm{NaMgF}_{3}$}

PL measurements were performed with the Jobin-Yvon FluoroLog ${ }^{\circledR}$. The PL spectrum of $\mathrm{NaMgF}_{3}: 0.2 \% \mathrm{Eu}$ is shown in Fig. 5.1, which consits of transitions from the $\mathrm{Eu}^{2+}$ ion. The sharp peak in the PL emission is due to the transition from the ${ }^{6} \mathrm{P}_{7 / 2}$ and ${ }^{6} \mathrm{P}_{5 / 2}$ excited states to the ${ }^{8} \mathrm{~S}_{7 / 2}$ ground state with an emission at $360 \mathrm{~nm}$, and the broad peak is from the band emission from the $4 \mathrm{f}^{6} 5 \mathrm{~d}\left(\mathrm{E}_{g}\right)$ excited states to the $4 \mathrm{f}^{7}$ ground state. In Fig. 5.1(b), it is shown that the $\mathrm{E}_{g}$ emission is at lower energies (red arrow) than the ${ }^{6} \mathrm{P}$ emission (blue arrow). The excited $4 f^{6} 5 \mathrm{~d}$ levels are split by the crystal field into two energy levels: the lower energy level $\mathrm{E}_{g}$ and a higher $\mathrm{T}_{2 g}$, which was also reported for $\mathrm{RbMgF}_{3}$ by Alcala et al. 82. The $4 \mathrm{f}^{7}$ excited state consists of two ${ }^{6} \mathrm{P}_{J}$ multiplets as illustrated in Fig. 5.1(b). These multiplets are shielded by the $5 \mathrm{~s}$ and $5 \mathrm{p}$ electrons and hence their interactions with the lattice are very weak. The ${ }^{6} \mathrm{P}_{J}$ transitions to the ground state, ${ }^{8} \mathrm{~S}_{7 / 2}$, are spin and parity forbidden in the electric dipole approximation. Therefore, this transition has a narrow PL line emission (Fig. 5.1(a)). Dotzler et al. reported that the line emission in the PL emission spectra is a zero phonon line which is due to the transition from the excited ${ }^{6} \mathrm{P}_{J}$ level to the ${ }^{8} \mathrm{~S}_{7 / 2}$ ground state. The smaller site peaks are Stokes and anti-Stokes vibronics which arise from higher ${ }^{6} \mathrm{P}_{J}$ level [4]. 


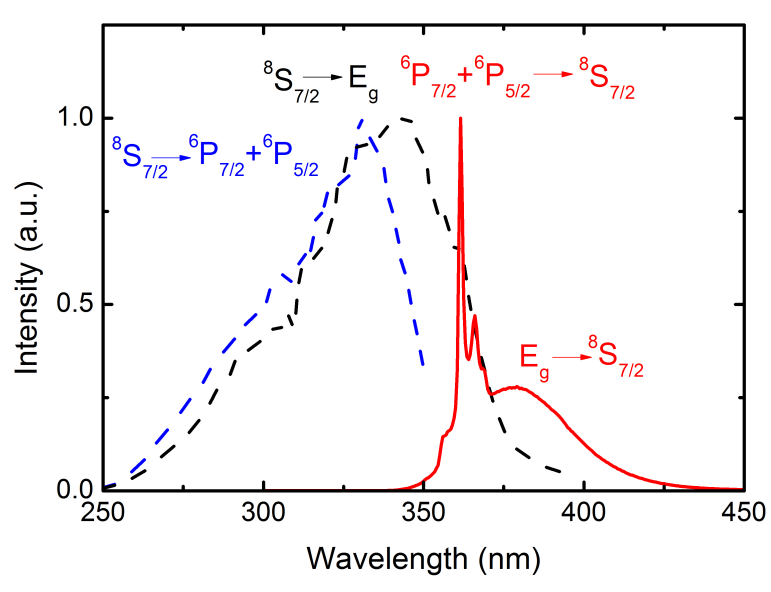

(a)

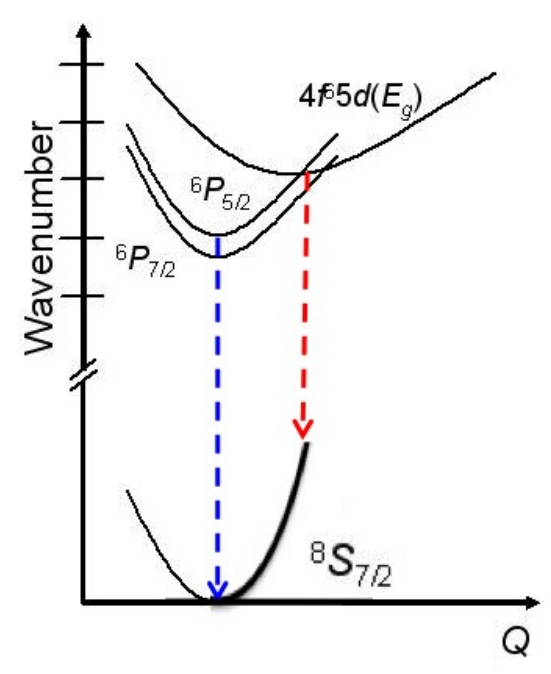

(b)

Figure 5.1: (a) PL excitation (dashed curve, $\lambda_{e m}=360$ (blue) $\mathrm{nm}$ and $420 \mathrm{~nm}$ (black)) and emission (solid curve, $\lambda_{e x}=256 \mathrm{~nm}$ ) spectra for $\mathrm{NaMgF}_{3} 0.2 \% \mathrm{Eu}$.

(b) Configuration coordinate diagram of $\mathrm{Eu}^{2+}$ in $\mathrm{NaMgF}_{3}$ [3, 9].

After measuring the PL emission and excitation spectra of $\mathrm{NaMgF}_{3}$ :Eu with the JobinYvon FluoroLog ${ }^{\circledR}$, the sample was heavily irradiated. Thus, the sample was mounted on a transportable holder for the PL measurements and the whole holder was placed in a light-tight box for transportation to the X-ray generator. The PL holder was placed in the $\mathrm{X}$-ray generator, which is operating at $40 \mathrm{~mA}$ and $40 \mathrm{kV}$, and the sample was irradiated up to an initial dose of $19 \mathrm{kGy}$. After radiation, the holder with sample was transported back in the light-tight box to the spectrometer and the PL measurement was repeated with the sample in the same position. Fig. 5.2(a) shows the absolute PL intensity of the emission spectra for $\mathrm{Eu}^{2+}$ before (solid curve) and after (dashed curve) irradiation. The PL intensity of the $\mathrm{Eu}^{2+}$ decreases after receiving a dose of $\sim 19 \mathrm{kGy}$ and the emission wavelengths of the $\mathrm{Eu}^{2+}$ transition remain unchange. Although a shift in the wavelengths was expected but this could not be detected with the spectrometer. The ratio of the $\mathrm{E}_{g}$ level to the ${ }^{6} \mathrm{P}_{J}$ excited states changes from 1.4:1 before irradiation to 2:1 after heavy irradiation. This is illustrated in the normalised PL plot in the inset of Fig. 5.2(a). The change of the ratio 
might be due to the creation of defects nearby the $\mathrm{Eu}^{2+}$ site. The $5 \mathrm{~d}$ emission seems to increase on top of the decreasing $\mathrm{Eu}^{2+}$ signal after heavy irradiation (Fig. 5.2(a)). Fig. 5.2 (b) shows the PL excitation and emission spectra of $\mathrm{Eu}^{3+}$ where the transitions from $\mathrm{Eu}^{3+}$ are indicated.

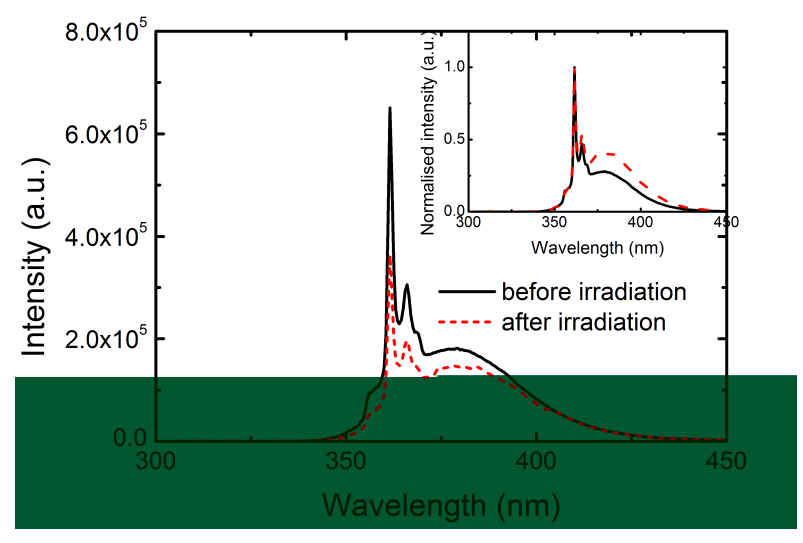

(a)

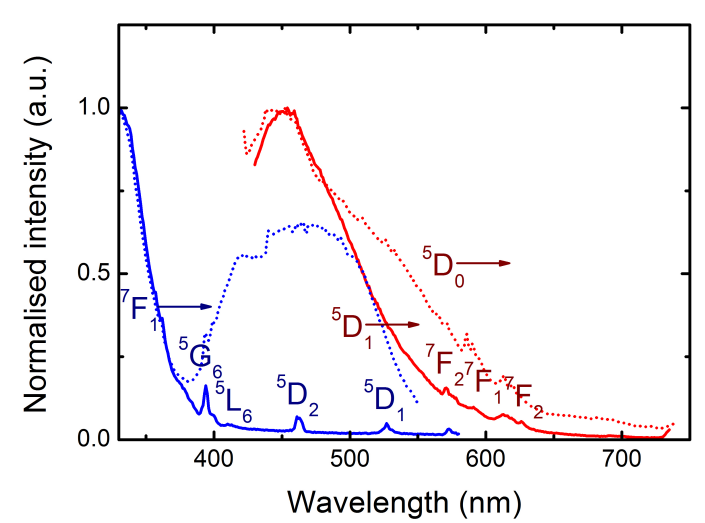

(b)

Figure 5.2: (a) Absolute PL intensity of the emission spectra, $\lambda_{e x}=256 \mathrm{~nm}$, for $\mathrm{NaMgF}_{3}$ $0.2 \%$ Eu before (solid curve) and after irradiation with a dose of 19 kGy (dashed curve). Inset: Normalised PL of the emission spectra, $\lambda_{e x}=256 \mathrm{~nm}$, for $\mathrm{NaMgF}_{3} 0.2 \% \mathrm{Eu}$ before (solid curve) and after irradiation with a dose of $\sim 19 \mathrm{kGy}$ (dashed curve).

(b) PL excitation at $\lambda_{e m}=610 \mathrm{~nm}$ (blue: solid curve - before irradiation, dashed curve - after irradiation with a dose of $\sim 19 \mathrm{kGy}$ ) and emission at $\lambda_{e x}=396 \mathrm{~nm}$ (red: solid curve - before irradiation, dashed curve - after irradiation (with 19 $\mathrm{kGy})$ ) spectra for the $\mathrm{Eu}^{3+}$ ion.

The normalised excitation spectrum measured at $610 \mathrm{~nm}$ before (solid curve) and after (dashed curve) irradiation are plotted in Fig. 5.3(a). The excitation spectrum before irradiation consist of the transition from the ${ }^{7} \mathrm{~F}_{1}$ level of $\mathrm{Eu}^{3+}$ and one peak centred at $340 \mathrm{~nm}$. After heavy irradiation, the intensity of the broad peak centred around $340 \mathrm{~nm}$ increases and a second peak centred at $470 \mathrm{~nm}$ appears (dashed curve in Fig. 5.3(a)). The 
transitions from the ${ }^{7} \mathrm{~F}_{1}$ level were not detected after irradiation because the intensity of the unknown defect centred at $470 \mathrm{~nm}$ overlaps the ${ }^{7} \mathrm{~F}_{1}$ transitions.

The $\mathrm{Eu}^{3+}$ emission spectra excited at $\lambda_{e x}=396 \mathrm{~nm}$ shows transitions from ${ }^{5} \mathrm{D}_{0}$ and ${ }^{5} \mathrm{D}_{1}$ levels (Fig. 5.2(b) and an additional large peak around $450 \mathrm{~nm}$ (see Fig. 5.3(b)). This new band at $450 \mathrm{~nm}$ can be assigned to some defects. The precise origin of these defects is unknown, but can probably be ascribed to the same defect observed in the nanoparticles and will be discussed further in the next chapter. However this defect with a peak at 450 $\mathrm{nm}$ could be from oxygen. After heavy irradiation, the intensity of the broad peak around $450 \mathrm{~nm}$ increases on top of an increase of the ${ }^{5} \mathrm{D}_{0} \rightarrow{ }^{7} \mathrm{~F}_{1},{ }^{7} \mathrm{~F}_{2}$ transitions $(\mathrm{Fig} 5.3(\mathrm{c}+\mathrm{d}))$. The ratio of ${ }^{7} \mathrm{~F}_{2}:{ }^{7} \mathrm{~F}_{1}$ changes from 2:1 before irradiation to 1:1 after heavy irradiation (Fig. $5.3(\mathrm{c}))$. The ${ }^{5} \mathrm{D}_{0} \rightarrow{ }^{7} \mathrm{~F}_{1}$ transition is a magnetic dipole transition and therefore almost independent of the crystalline structure as discussed by Görller-Walrand and Binnemans [58. Whereas the ${ }^{5} \mathrm{D}_{0} \rightarrow{ }^{7} \mathrm{~F}_{2}$ transition is a hypersensitive transition and so very dependent on the crystalline environment. This means before irradiation the environment was highly distorted because the ratio of ${ }^{7} \mathrm{~F}_{2}:{ }^{7} \mathrm{~F}_{1}$ was $2: 1$. The reason for this could be that the charge balance requires the existence of $\mathrm{V}_{k}$ or $\mathrm{Na}$ vacancies $\left(\mathrm{V}_{A}\right.$ centres), which can be close to the $\mathrm{Eu}^{3+}$ ions. The average $\mathrm{Eu}^{3+}$ ions of the newly created $\mathrm{Eu}^{3+}$ ions are less distorted than the already existing ones, which is shown in the decreases of the ${ }^{7} \mathrm{~F}_{2}:{ }^{7} \mathrm{~F}_{1}$ ratio. Hence, there are two $\mathrm{Eu}^{3+}$ in the crystal after irradiation of $\sim 19 \mathrm{kGy}$ : one distorted site and one less distorted site.

After the irradiation, the average crystal environment of $\mathrm{Eu}^{3+}$ was less distorted because the ratio of ${ }^{7} \mathrm{~F}_{2}:{ }^{7} \mathrm{~F}_{1}$ changes from $2: 1$ to $1: 1$. The reason for this might be the additional generation of $\mathrm{H}$ - or $\mathrm{F}$ - centres by the X-rays, which do not have to be near the $\mathrm{Eu}^{3+}$ sites (Fig. 5.3 (c)). To measure the change of ratio of these transitions, the unknown defect was subtracted from the PL data by creating a baseline with the OriginLab program. Fig. 5.3 (d) shows the PL excitation spectra measured at $450 \mathrm{~nm}$ before (solid curve) and after (dashed curve) irradiation. The new band at $450 \mathrm{~nm}$ cannot be attributed to the ${ }^{8} \mathrm{~S}_{7 / 2} \rightarrow$ $\mathrm{E}_{g}$ transition because the emission from to the $\mathrm{E}_{g}$ level has a peak from $250 \mathrm{~nm}$ until 400 
$\mathrm{nm}$ as shown in Fig. 5.1. The peak intensity of the defect centred at $340 \mathrm{~nm}$ increases after heavy irradiation.

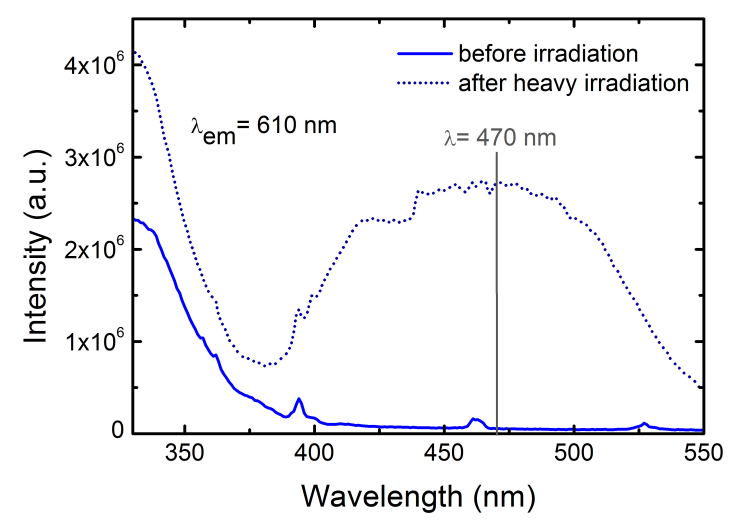

(a)

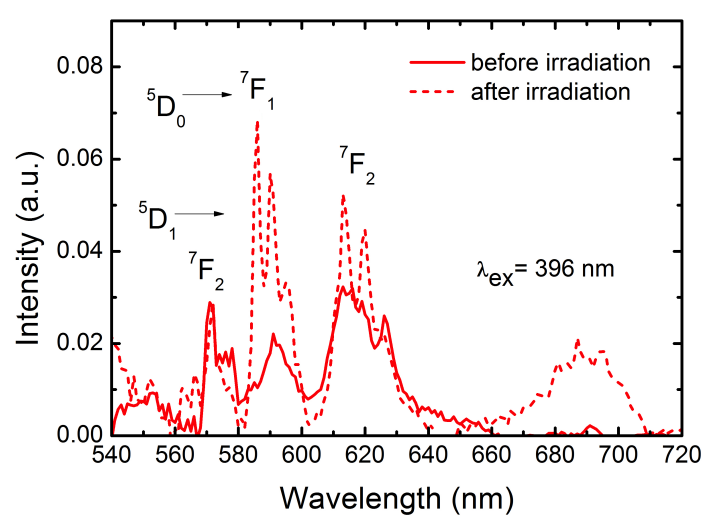

(c)

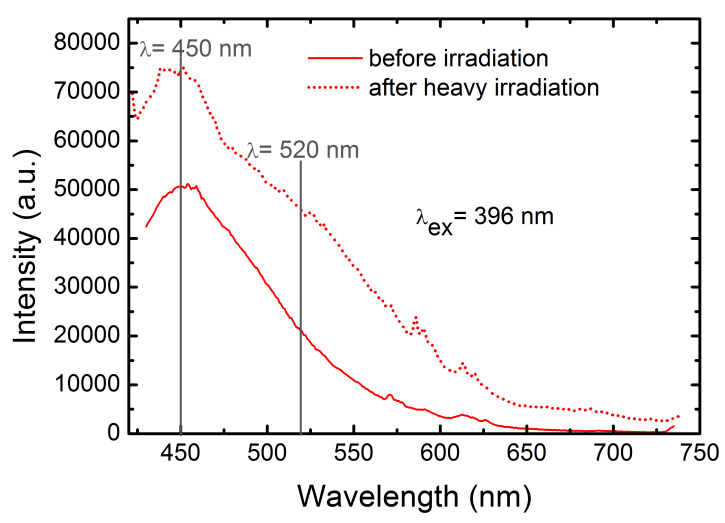

(b)

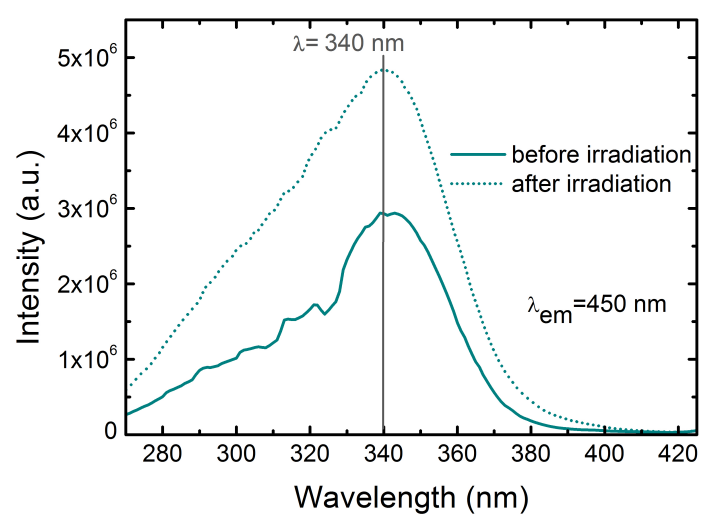

(d)

Figure 5.3: (a) Absolute PL intensity of the excitation spectra, $\lambda_{e m}=610 \mathrm{~nm}$, before (solid curve) and after irradiation with a dose of $\sim 19 \mathrm{kGy}$ (dashed curve).

(b) Absolute PL intensity of the emission spectra, $\lambda_{e x}=396 \mathrm{~nm}$, before (solid curve) and after irradiation with a dose of $\sim 19 \mathrm{kGy}$ (dashed curve).

(c) PL emission spectra of $\mathrm{Eu}^{3+}$ corrected for the broad unknown peak before (solid curve) and after heavy irradiation with a dose of $\sim 19 \mathrm{kGy}$ (dashed curve). (d) Absolute PL excitation spectra at $\lambda_{e m}=450 \mathrm{~nm}$ before (solid curve) and after heavy irradiation with a dose of $\sim 19$ kGy (dashed curve). 
Radiation damage might lead to defects near the $\mathrm{Eu}^{2+}$ ions. This could change the crystal field and lead to a shift in the $5 \mathrm{~d} 4 \mathrm{f}$ level so that the energy difference between the $5 \mathrm{~d} 4 \mathrm{f}$ and excited $4 \mathrm{f}$ levels is smaller (Fig. 5.4(a)). Fig. 5.4(b) illustrates the increase of the $\mathrm{Eu}^{3+}$ signal. Due to the damage induced by radiation, more defects are generated near to the $\mathrm{Eu}^{2+}$ ions. An electron which is stimulated from the $\mathrm{Eu}^{2+}$ site recombines in a very deep non-optical or non-thermal stimulated trap. Hence, more $\mathrm{Eu}^{3+}$ will be detected.

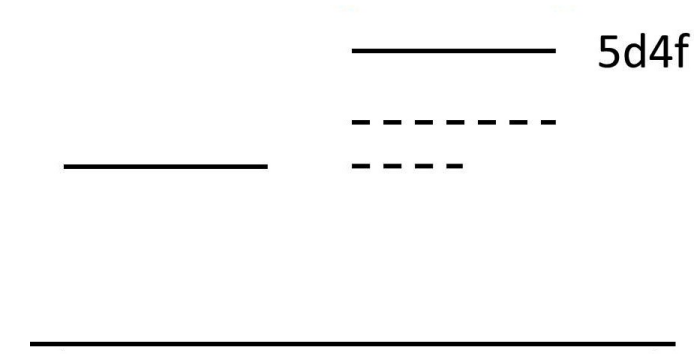

(a)

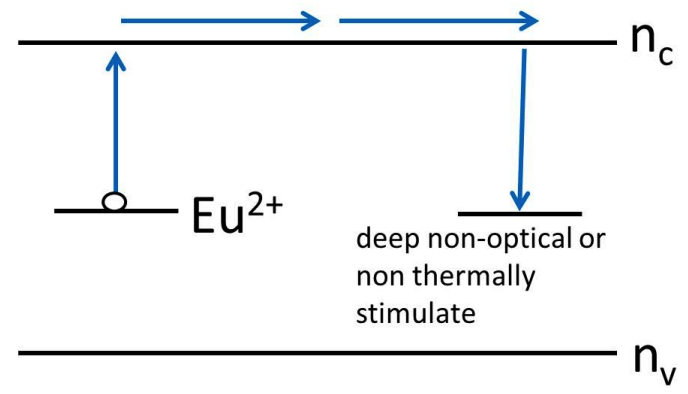

(b)

Figure 5.4: (a) Change of the crystal field leading to a shift in the 5d4f level in $\mathrm{Eu}^{2+}$.

(b) Electron from $\mathrm{Eu}^{2+}$ recombines in a deep non-optical or non-thermal trap.

Fig. 5.5 shows the PL lifetime decay curve (solid curve) with the exponential fit (dashed curve) for the $0.2 \%$ Eu doped $\mathrm{NaMgF}_{3}$ same at $\lambda_{e m}=360 \mathrm{~nm}$ (a) and (b) $\lambda_{e m}=390 \mathrm{~nm}$, when excited at $\lambda_{e x}=256 \mathrm{~nm}$. 


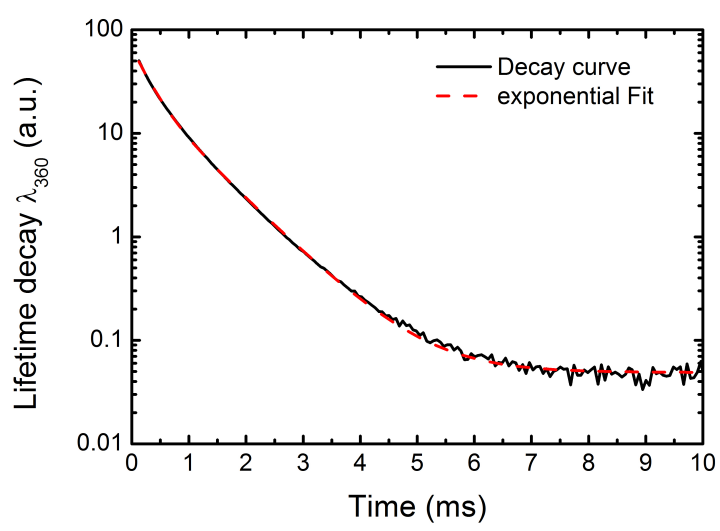

(a)

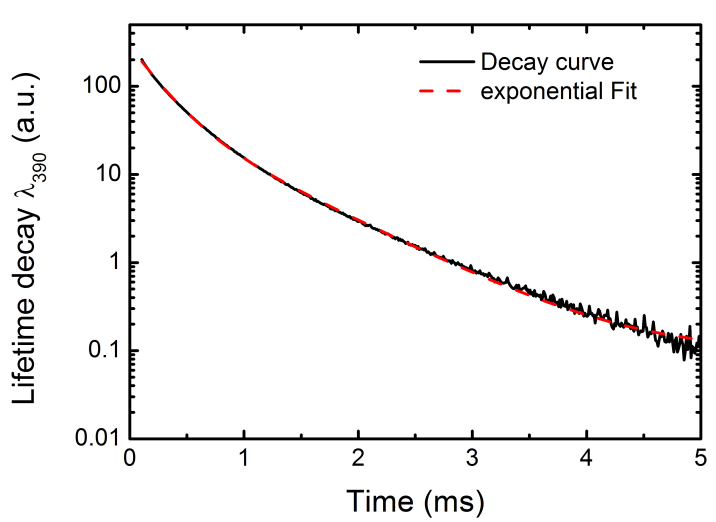

(b)

Figure 5.5: (a) PL lifetime decay of $0.2 \% \mathrm{Eu}$ doped $\mathrm{NaMgF}_{3}$ at $\lambda_{\text {em }}=360 \mathrm{~nm}$ (solid curve) and (b) $\lambda_{e m}=390 \mathrm{~nm}$ emission (solid curve). The dashed curve is the exponential fit. Both lifetimes were measured at $\lambda_{e x}=256 \mathrm{~nm}$.

The average PL lifetimes for the $0.2 \% \mathrm{Eu}^{2+}$ ions were measured with the Cary 50 . The PL lifetime for the $\mathrm{Eu}^{3+}$ and the unknown defect were measured with the Jobin-Yvon FluoroLog ${ }^{\circledR}$ spectrometer. The PL lifetime remains unchanged after heavy irradiation. The PL decay could be fitted two-exponential which can be described by two exponential components,

$$
I(t)=A_{1} \exp \left(\frac{-t}{\tau_{1}}\right)+A_{2} \exp \left(\frac{-t}{\tau_{2}}\right)+I_{0}
$$

For an emission wavelength of $360 \mathrm{~nm}$ the calculated average lifetime was $0.63 \pm 0.06 \mathrm{~ms}$, while for a wavelength of $390 \mathrm{~nm}$ a lifetime of $0.42 \pm 0.04 \mathrm{~ms}$ was obtained $\left(\lambda_{e x}=256 \mathrm{~nm}\right)$. The reason for the different lifetimes is already discussed in section [?]. The average lifetime was estimated from [67],

$$
\tau_{\text {ave }}=\left[\int t I(t) d t / \int I(t) d t\right]
$$

For an emission wavelength of $592 \mathrm{~nm}$ the calculated average lifetime was $11.1 \pm 1.1 \mathrm{~ms}$ $\left(\lambda_{e x}=375 \mathrm{~nm}\right)$ and it was measured with the Jobin-Yvon FluoroLog ${ }^{\circledR}$. The unknown defect centred at $\sim 460 \mathrm{~nm}$ had an average PL lifetime of $67 \pm 7 \mathrm{~ms}\left(\lambda_{e x}=375 \mathrm{~nm}\right)$. The PL lifetime of the unknown defect could only be fitted with a three exponential decay as 
summarised in Tab. 5.2. The lifetime of the unknown defect is large compared to the measured PL lifetimes of the Eu ions. This new band at $450 \mathrm{~nm}$ can be assigned to some defects. The precise origin of these defects is unknown and it cannot arise from the $\mathrm{Eu}^{2+}$ or $\mathrm{Eu}^{3+}$ transitions. Maybe the unknown defect is from oxygen as discussed by Williams et al. [10. The different compounds of the average PL lifetimes are listed in Tab. 5.1. The average lifetime measured for an emission wavelength of $360 \mathrm{~nm}$ showed two separate sites with different lifetimes of $0.28 \pm 0.03 \mathrm{~ms}$ and $0.85 \pm 0.09 \mathrm{~ms}$. For an emission wavelength of $390 \mathrm{~nm}$ the lifetime decay shows distributions of two sites with calculated lifetimes of $0.22 \pm 0.02 \mathrm{~ms}$ and $0.69 \pm 0.07 \mathrm{~ms}$. The lifetime measured at $592 \mathrm{~nm}$ shows a big distribution of lifetimes where one site has a lifetime of $2.0 \pm 0.2 \mathrm{~ms}$ and the other one shows a lifetime of $16.7 \pm 1.7 \mathrm{~ms}$. There are three sites calculated of the PL lifetime in the unknown defect with lifetimes of $5.2 \pm 0.5 \mathrm{~ms}, 33.2 \pm 3.3 \mathrm{~ms}$ and $107 \pm 11 \mathrm{~ms}$.

Table 5.1: Different compounds with the pre factors of the PL lifetimes of $0.2 \%$ Eu doped $\mathrm{NaMgF}_{3}$.

\begin{tabular}{|l|l|l|l|l|l|l|}
\hline$\lambda_{e m}(\mathrm{~nm})$ & factor $\mathrm{A}_{1}$ & $\tau_{1}(\mathrm{~ms})$ & factor $\mathrm{A}_{2}$ & $\tau_{2}(\mathrm{~ms})$ & factor $\mathrm{A}_{3}$ & $\tau_{3}(\mathrm{~ms})$ \\
\hline \hline 360 & 42 & $0.28 \pm 0.03$ & 26 & $0.85 \pm 0.09$ & & \\
\hline 390 & 233 & $0.22 \pm 0.02$ & 54 & $0.69 \pm 0.07$ & & \\
\hline 500 & 46 & $5.2 \pm 0.5$ & 6 & $33.2 \pm 3.3$ & 5 & $107 \pm 11$ \\
\hline 592 & 236 & $2.0 \pm 0.2$ & 47 & $16.7 \pm 1.0$ & & \\
\hline
\end{tabular}

Table 5.2: Average PL lifetimes of $0.2 \%$ Eu doped $\mathrm{NaMgF}_{3}$.

\begin{tabular}{|l|l|}
\hline$\lambda_{\text {em }}(\mathrm{nm})$ & $\tau_{\text {ave }}(\mathrm{ms})$ \\
\hline \hline 360 & $0.63 \pm 0.06$ \\
\hline 390 & $0.42 \pm 0.04$ \\
\hline 500 & $11.1 \pm 1.1$ \\
\hline 592 & $67 \pm 7$ \\
\hline
\end{tabular}




\subsubsection{Mn doped polycrystalline $\mathrm{NaMgF}_{3}$}

PL emission and excitation spectra for $\mathrm{NaMgF}_{3}$ samples doped with $1 \% \mathrm{Mn}$ are shown in Fig. 5.6. The PL emission spectrum was excited at $396 \mathrm{~nm}$ (see Fig. 5.6(b)). The spectrum consists of two peaks: one at $500 \mathrm{~nm}$ and the other one at $600 \mathrm{~nm}$ (solid curve). Fig. 5.6(b) shows the PL emission spectrum of the $0.5 \%$ Mn doped $\mathrm{NaMgF}_{3}$ sample (dashed curve). The intensity of the peak centred at $500 \mathrm{~nm}$ has nearly the same intensity as the peak of the regular $\mathrm{Mn}^{2+}$ site centred at $600 \mathrm{~nm}$. This is different for the $1 \% \mathrm{Mn}$ doped sample where the ratio of the intensity of the distorted peak is smaller compared to the intensity of the regular peak (Fig. 5.6(b)). The change in ratio of the regular site compared to the distorted site is 1:1 for the $0.5 \%$ Mn doped sample and 3:1 for the $1 \%$ doped Mn sample. The reason for the change could be the existence of fewer distorted defects in the sample with the higher concentration. The PL excitation spectrum for the $600 \mathrm{~nm}$ emission (solid curve in Fig. 5.6(a)) can be attributed to transitions to the ${ }^{6} \mathrm{~A}_{1 g}$ ground state and the excited state levels are indicated in Fig. 5.6(a). The two $\mathrm{Mn}^{2+}$ sites plotted in Fig. 5.6(b) are unexpected because the space group of $\mathrm{NaMgF}_{3}$ is $P_{b n m}$ and $\mathrm{Mn}^{2+}$ is believed to substitute for the $\mathrm{Mg}$ site. Thus, PL emission from only one $\mathrm{Mn}^{2+}$ site is expected. 


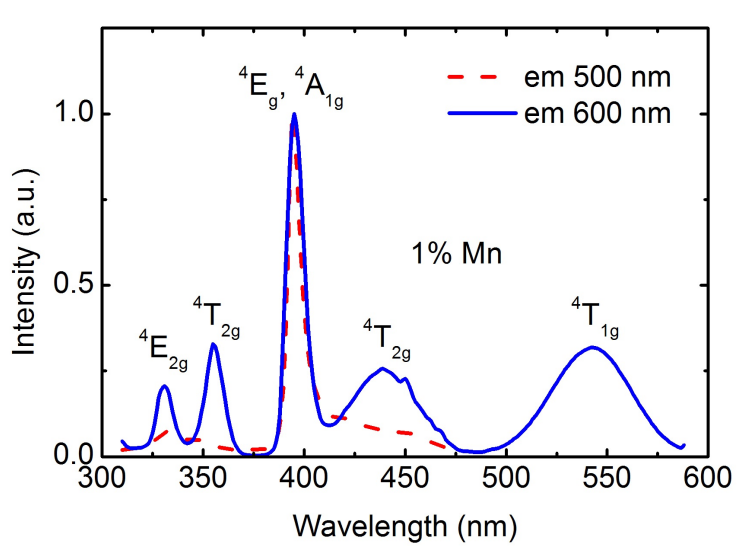

(a)

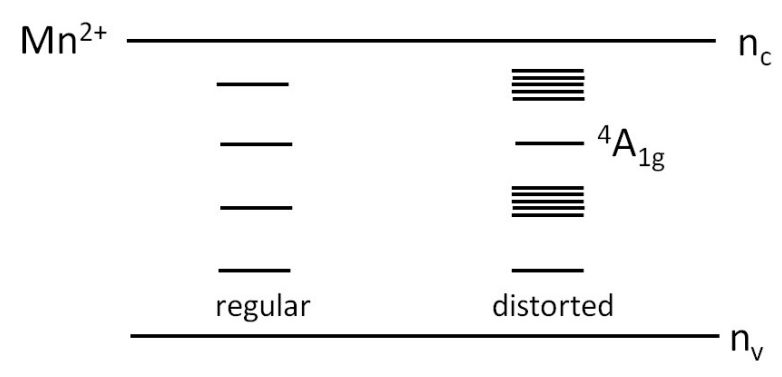

(c)

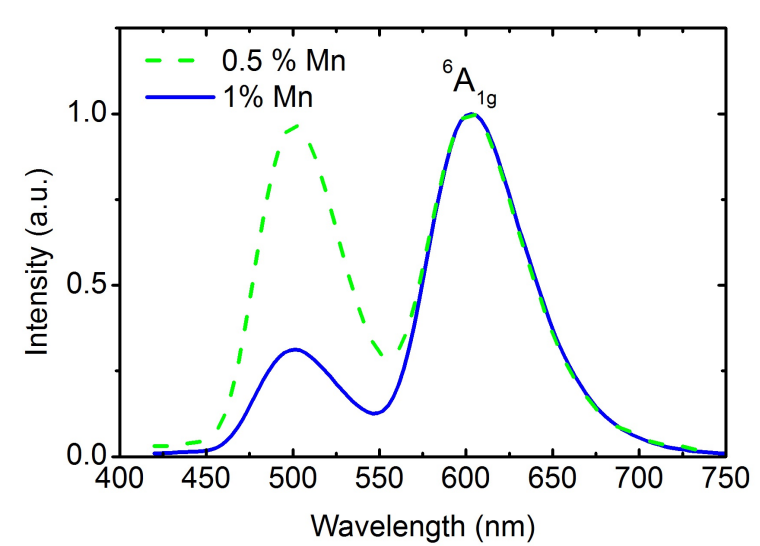

(b)

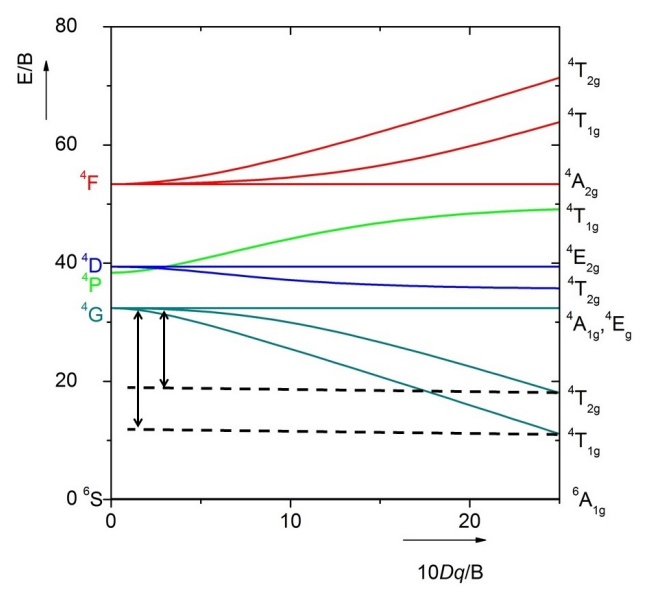

(d)

Figure 5.6: (a) PL excitation spectrum at $\lambda_{e m}=500 \mathrm{~nm}$ (dashed curve) and $\lambda_{e m}=600 \mathrm{~nm}$ emission (solid curve) for the $1 \% \mathrm{Mn}$ doped $\mathrm{NaMgF}_{3}$ sample.

(b) PL emission spectrum for $\mathrm{NaMgF}_{3}$ doped with $0.5 \% \mathrm{Mn}$ (dashed curve) and $1 \% \mathrm{Mn}$ (solid curve) excited at $396 \mathrm{~nm}$.

(c) Broadening of the peaks in the PL excitation spectra for the distorted $\mathrm{Mn}^{2+}$ site.

(d) Splitting of the energy levels of $\mathrm{Mn}^{2+}$ in an octahedral crystal field.

The PL excitation spectrum from the $500 \mathrm{~nm}$ emission was also measured and it is plotted 
in Fig. 5.6(a) (dashed curve). The peak centred at $\sim 396 \mathrm{~nm}$ can be attributed to the $\mathrm{Mn}^{2+}{ }^{6} \mathrm{~A}_{1 g}$ and ${ }^{4} \mathrm{E}_{g}$ levels. All of the distorted $\mathrm{Mn}^{2+}$ transitions are significantly broader. The broadening of the excitation spectra for the $500 \mathrm{~nm}$ emission can be explained with a model illustrated in Fig. 5.6(c). The regular $\mathrm{Mn}^{2+}$ site shows distinct peaks in the excitation spectra, which can be distinguished in the PL. The distorted site shows traps that are close to each other resulting in transitions that cannot be distinguished in the PL excitation spectrum. Therefore, the peaks are broader in the PL excitation spectrum for the second $\mathrm{Mn}^{2+}$ site centred at $500 \mathrm{~nm}$. Due to the crystal field, the ${ }^{4} \mathrm{E}_{2 g},{ }^{4} \mathrm{~T}_{2 g}$ and ${ }^{4} \mathrm{~T}_{1 g}$ levels shift with the crystal field parameter and the ${ }^{4} \mathrm{~A}_{1 g}$ and $\mathrm{E}_{g}$ levels are not affected by the crystal field (Fig. 5.6(d)). Therefore, the ${ }^{4} \mathrm{~A}_{1 g}$ and $\mathrm{E}_{g}$ levels still show a distinct peak and the other levels are broader. Therefore, it is likely that the $500 \mathrm{~nm}$ PL emission comes from a distorted $\mathrm{Mn}^{2+}$ site. There were no additional peaks before and after heavy irradiation with $\sim 12 \mathrm{kGy}$ observed and these measurements were explicit discussed in the Ph.D. thesis of Christian Dotzler [3].

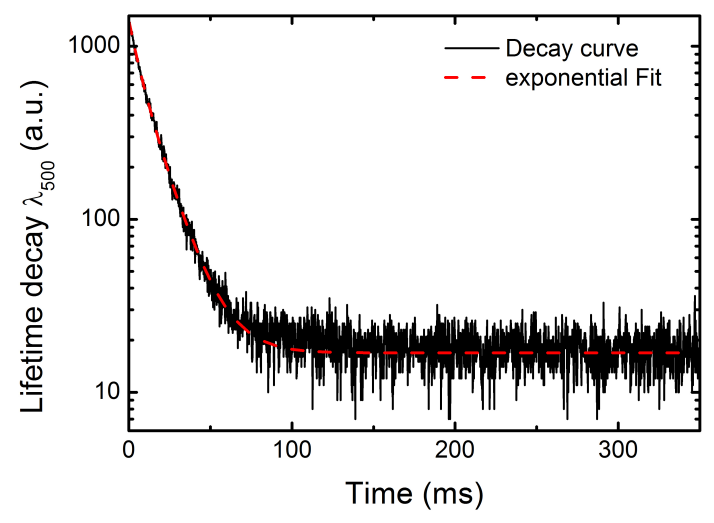

(a)

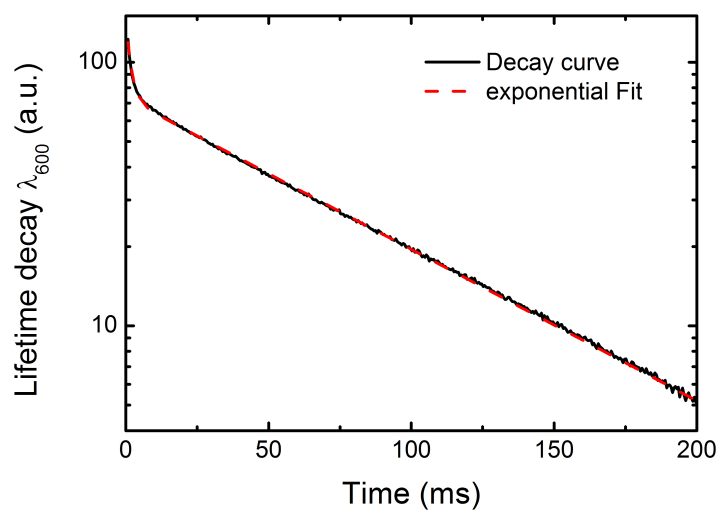

(b)

Figure 5.7: (a) PL lifetime decay of $1 \% \mathrm{Mn}$ doped $\mathrm{NaMgF}_{3}$ at $\lambda_{e m}=500 \mathrm{~nm}$ (solid curve) and (b) $\lambda_{e m}=600 \mathrm{~nm}$ emission (solid curve). The dashed curve is the exponential fit. Both lifetimes were measured at $\lambda_{e x}=465 \mathrm{~nm}$.

Fig. 5.4 shows the PL lifetime decay curve (solid curve) with the exponential fit (dashed 
curve) for the $1 \% \mathrm{Mn}$ doped $\mathrm{NaMgF}_{3}$ measured at $\lambda_{e m}=500 \mathrm{~nm}$ (a) and (b) $\lambda_{e m}=600$ $\mathrm{nm}\left(\lambda_{e x}=465 \mathrm{~nm}\right)$. The lifetimes of the Mn doped samples were measured with the JobinYvon FluoroLog ${ }^{\circledR}$. They could be fitted in a two exponential decay and the distribution of the lifetimes are listed in Tab. 5.3. Both $\mathrm{Mn}^{2+}$ sites have two compounds of the PL lifetime: a shorter lifetime which is from a more distorted site and a longer lifetime from a less distorted site.

Table 5.3: Different compounds with the pre factors of the PL lifetimes of Mn doped $\mathrm{NaMgF}_{3}$.

\begin{tabular}{|l|l|l|l|l|}
\hline$\lambda_{e m}(\mathrm{~nm})$ & factor $\mathrm{A}_{1}$ & $\tau_{1}(\mathrm{~ms})$ & factor $\mathrm{A}_{2}$ & $\tau_{2}(\mathrm{~ms})$ \\
\hline \hline $0.5 \% 500$ & 8342 & $12.2 \pm 1.2$ & 226 & $30.9 \pm 3.1$ \\
\hline $0.5 \% 600$ & 2179 & $52.8 \pm 5.3$ & 7776 & $104 \pm 10$ \\
\hline $1 \% 500$ & 505 & $5.9 \pm 0.6$ & 880 & $14.4 \pm 1.4$ \\
\hline $1 \% 600$ & 11105 & $53.9 \pm 5.4$ & 24219 & $101 \pm 10$ \\
\hline
\end{tabular}

For the regular site of $\mathrm{Mn}$ in the $\mathrm{NaMgF}_{3}$ an average lifetime of $98 \pm 10 \mathrm{~ms}$ and $91.4 \pm 9.1 \mathrm{~ms}$ for the $0.5 \%$ and the $1 \%$ doped sample was calculated $\left(\lambda_{e m}=600 \mathrm{~nm}\right)$, respectively. An average PL lifetime of $13.5 \pm 1.4 \mathrm{~ms}$ and $12.8 \pm 1.3 \mathrm{~ms}$ was measured for the distorted $\mathrm{Mn}^{2+}$ site for the $0.5 \%$ and $1 \%$ doped sample $\left(\lambda_{e m}=500 \mathrm{~nm}\right)$, respectively. The transitions of the regular $\mathrm{Mn}^{2+}$ site are spin and parity forbidden. F-centres next to the regular $\mathrm{Mn}^{2+}$ site make this transition only parity forbidden resulting in a high average PL lifetime. The distorted $\mathrm{Mn}^{2+}$ site is only parity forbidden and it shows a distribution of the crystal fields. This results in a mixing of crystal field symmetry and therefore, more defects near this site. Thus, the distorted site of $\mathrm{Mn}^{2+}$ shows a shorter measured PL lifetime due to the shift in the levels, which is illustrated in Fig. 5.6(d). The PL lifetime of the regular site decreases by $10 \%$ with the increasing Mn concentration. This can be a result of the energy transfer between the $\mathrm{Mn}^{2+}$ site and the recombination at a non-radiative recombination centre. The probability of this energy transfer is proportional to the distance of the atoms which 
is depending on the Mn concentration, $\frac{1}{r^{6}} \sim \frac{1}{\text { conc }^{2}}$. This model was used to explain the decrease of the average PL lifetimes in $\mathrm{Eu}^{3+}$ doped samples [11, 12, 22, 64, 83, 84, 85, 86].

Table 5.4: Average PL lifetimes of distorted and regular $\mathrm{Mn}^{2+}$ sites in $\mathrm{NaMgF}_{3}$.

\begin{tabular}{|l|l|l|}
\hline Mn concentration $(\%)$ & $\tau_{500 n m}(\mathrm{~ms})$ & $\tau_{600 n m}(\mathrm{~ms})$ \\
\hline \hline 0.5 & $13.5 \pm 1.4$ & $98 \pm 10$ \\
\hline 1 & $12.8 \pm 1.3$ & $91.4 \pm 9.1$ \\
\hline
\end{tabular}

\subsection{Thermoluminescence measurements}

The TL measurements were performed with a custom-built TL spectrometer in the manner explained in section 4.3 .

\subsubsection{Eu doped polycrystalline $\mathrm{NaMgF}_{3}$}

The $\mathrm{NaMgF}_{3}$ sample doped with $0.2 \%$ Eu was irradiated for with a Philips X-ray generator operating at $40 \mathrm{kV}$ and $40 \mathrm{~mA}$, using a W-tube with an Aluminium filter. The glow curves were obtained using a heating rate of $1 \mathrm{~K} / \mathrm{s}$ and an irradiation time of 1 minute was used when the data was collected using a PMT and $5 \mathrm{~min}$ for the CCD. The advantage of collecting the data with a CCD detector is that it is possible to distinguish the wavelengths of the TL transitions. The TL spectrum is plotted in Fig. $5.8(\mathrm{a}+\mathrm{b})$ and the activation energies of the peaks of the glow curves are summarised in Tab. 5.5. They were obtained using first order kinetics glow curve deconvolution software and by minimizing the number of peaks during fitting of the glow curve (see also Fig. 5.8(a) - dashed curves). Fig. 5.8(b) shows the TL intensity plotted against the wavelength for different temperatures. The units for the TL intensity in Fig. 5.8 $(\mathrm{a}+\mathrm{b})$ are different because for the deconvolution the whole peak from $360 \mathrm{~nm}$ to $420 \mathrm{~nm}$ was integrated and hence the y-axis in Fig. 5.8(a) shows a higher TL intensity than the peak maximum at $360 \mathrm{~nm}$ in Fig. 5.8(b). Potential 
additional peaks above $620 \mathrm{~K}$ could not be detected due to the temperature limitation of the equipment used.

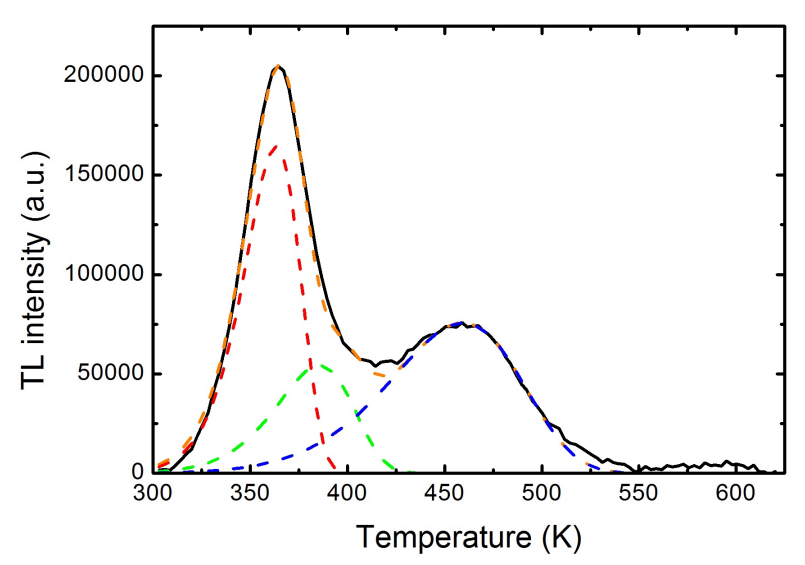

(a)

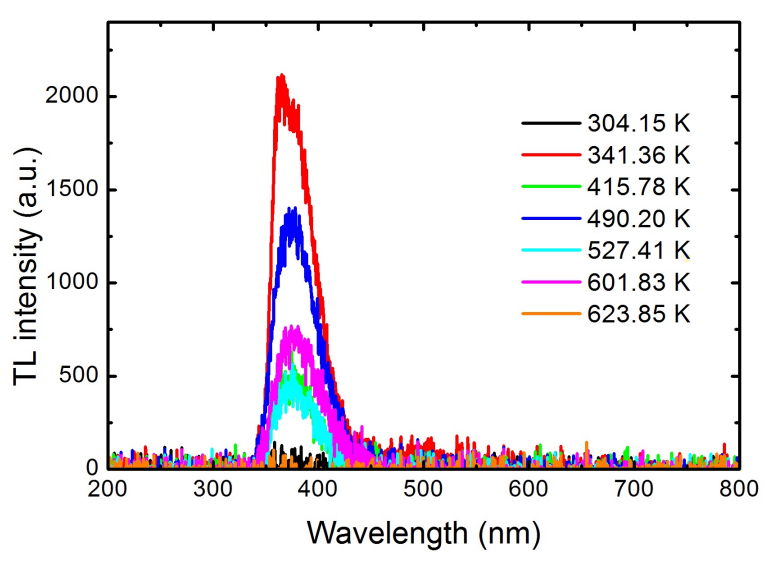

(b)

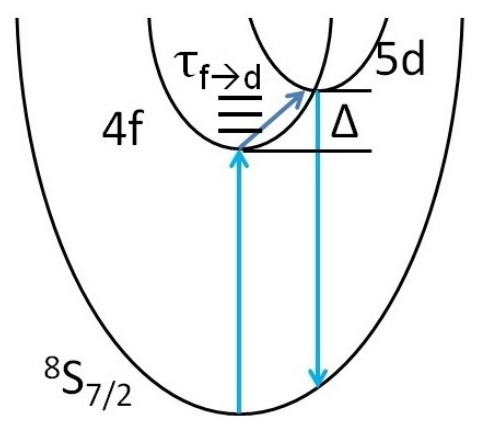

(c)

Figure 5.8: (a) TL for the $\mathrm{NaMgF}_{3}$ sample doped with $0.2 \% \mathrm{Eu}$ (solid curve). The TL data was detected with the CCD after 5 min X-ray irradiation $(40 \mathrm{kV}, 40 \mathrm{~mA})$ at a heating rate of $1 \mathrm{~K} / \mathrm{s}$. The fitted peaks were obtained using first order kinetics glow curve deconvolution (dashed curves).

(b) TL spectra vs. wavelength for the different temperatures.

(c) Model for the probability of the emission from the $4 \mathrm{f}$ and $5 \mathrm{~d}$ levels to the ground states for higher temperatures. 
Table 5.5: The activation energies of the individual glow curve peaks for the $\mathrm{NaMgF}_{3}: 0.2$ $\%$ Eu sample obtained by first order kinetics glow curve deconvolution. The top line is the peak temperature for the glow curve.

\begin{tabular}{|l|l|l|l|}
\hline & $363 \mathrm{~K}$ & $386 \mathrm{~K}$ & $459 \mathrm{~K}$ \\
\hline \hline $\begin{array}{l}0.2 \% \mathrm{Eu} \\
\text { peak height }\end{array}$ & $0.76 \pm 0.07 \mathrm{eV}$ & $0.61 \pm 0.06 \mathrm{eV}$ & $0.50 \pm 0.05 \mathrm{eV}$ \\
164000 & 54000 & 76000 \\
\hline
\end{tabular}

The TL signal arises mainly from the $\mathrm{Eu}^{2+}$ transition (see Fig. 5.8(b)). The $5 \mathrm{~d}$ emission seems to increase with increasing temperature as shown in Fig. 5.8(a). The probability for the transition between the $4 \mathrm{f}$ and $5 \mathrm{~d}$ level, $\tau_{f \rightarrow d}$, is dependent on the energy difference between the $\mathrm{d} \rightarrow \mathrm{f}$ level, $\Delta$, and the temperature, $\mathrm{T}$. This can be estimated from the following equation,

$$
\tau_{f \rightarrow d}=\tau_{o} e^{-\frac{\Delta}{k T}}
$$

where $\mathrm{k}$ is the Boltzmann's constant. This means that with increasing temperature the energy difference between the $4 \mathrm{f}$ and $5 \mathrm{~d}$ level is decreasing and this will result in a higher probability for the transition from the $4 \mathrm{f}$ to the $5 \mathrm{~d}$ levels. Therefore, at higher temperatures more of the $4 \mathrm{f}$ levels emission will be quenched by the $5 \mathrm{~d}$ level resulting in an increase of the emission from the $5 \mathrm{~d}$ level. Therefore, there is hardly any line emission detected in the TL spectra (Fig. 5.8(c)). The energy difference, $\Delta$, between the $\mathrm{d} \rightarrow \mathrm{f}$ level is around $9700 \mathrm{~cm}^{-1}(1.2 \mathrm{eV})$. The value $\Delta / \mathrm{k}$ can be estimated from,

$$
\frac{\Delta}{k}=1.39 \times 10^{4} \mathrm{~K}
$$

Another possibility of a decrease in the line emission with increasing temperature is the probability of exciting into the $5 \mathrm{~d}$ level becomes more likely with increasing temperature. The glow curve consists out of two main peaks located at $363 \mathrm{~K}$ and $459 \mathrm{~K}$. There is also a weaker peak detected at $385 \mathrm{~K}$. The activation energies of the peaks centred at $363 \mathrm{~K}$, $386 \mathrm{~K}$ and $459 \mathrm{~K}$ are $0.76 \mathrm{eV}, 0.61 \mathrm{eV}$ and $0.5 \mathrm{eV}$, respectively. 


\subsubsection{Mn doped polycrystalline $\mathrm{NaMgF}_{3}$}

For the $\mathrm{NaMgF}_{3}$ doped with $\mathrm{Mn}^{2+}$ the TL spectrum was measured for the $0.5 \%$ and 1 \% sample (Fig 5.9-5.11). The TL spectrum for the $0.5 \%$ sample was measured using a heating rate of $1 \mathrm{~K} / \mathrm{s}$ when irradiating for $1 \mathrm{~min}$ with the X-ray generator $(40 \mathrm{kV}, 40 \mathrm{~mA})$, as plotted in Fig. 5.9 (solid curve) and the first order kinetics glow curve deconvolution (dashed curve). The glow curve peaks were summarised in Tab. 5.6. The TL emission arises from the ${ }^{6} \mathrm{~A}_{1 g}$ emission at $600 \mathrm{~nm}$, plotted in Fig. 5.9(b) and Fig. 5.10(e). No TL emission arises from the distorted $\mathrm{Mn}^{2+}$ site which was detected at $500 \mathrm{~nm}$ in the PL emission spectrum. A reason could be that a F-centres might be closer to the regular $\mathrm{Mn}^{2+}$ site and more likely to recombine with this site. The electron from the F-centre is stimulated into the conduction band and gets trapped in the excited $\mathrm{Mn}^{2+*}$, as shown in Fig $5.9(\mathrm{~d})$. The detrapping out of this $\mathrm{Mn}^{2+*}$ site leads to a higher TL signal of the regular site. Another reason could be that the distorted site might show a non-radiative recombination. Fig. 5.9 (c) shows the normalised TL emission curve at $443 \mathrm{~K}$ (blue curve) and $622 \mathrm{~K}$ (black curve). It was observed that the maximum of the TL emission shifts towards higher energies with increasing temperature and the peak broadens with increasing temperature. The shift is from $595 \mathrm{~nm} \rightarrow 588 \mathrm{~nm}$ for the $0.5 \% \mathrm{Mn}$ doped sample. This phenomenon is similar to the process of the temperature dependence of the bandgap of semiconductors [87, 88, 89, 90]. 


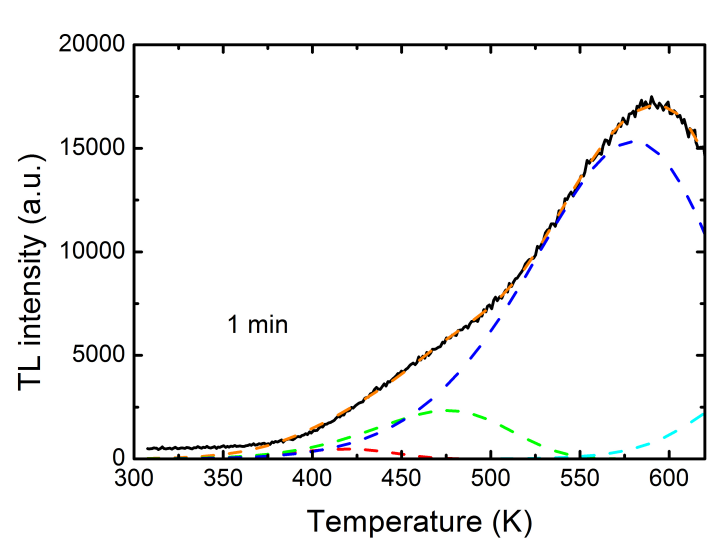

(a)

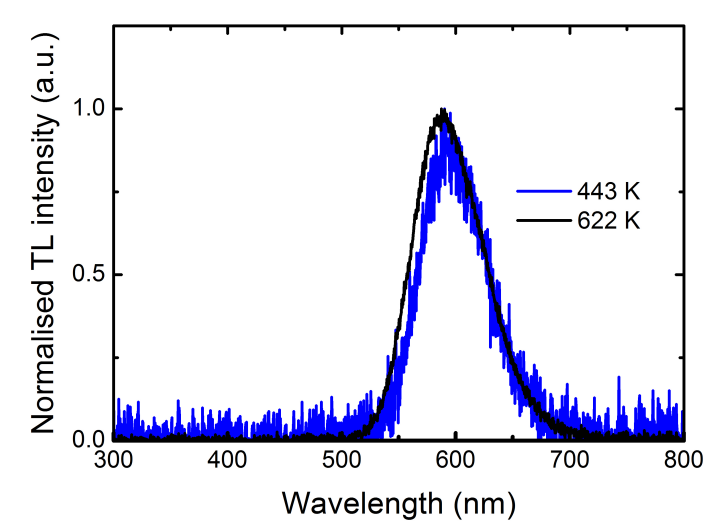

(c)

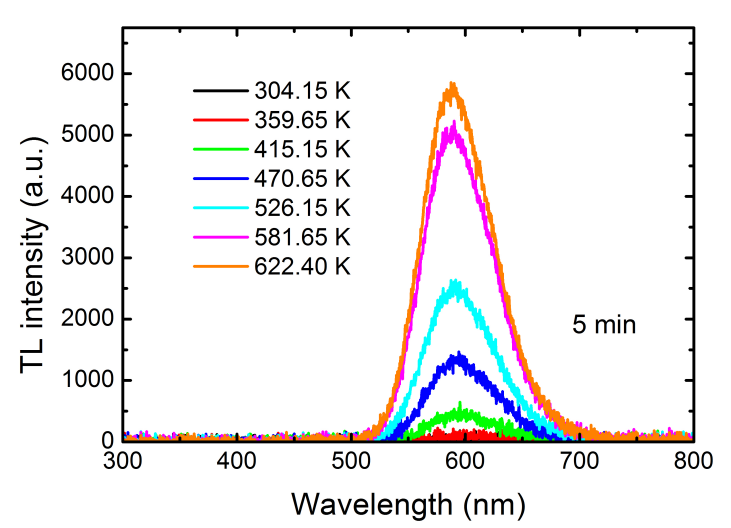

(b)

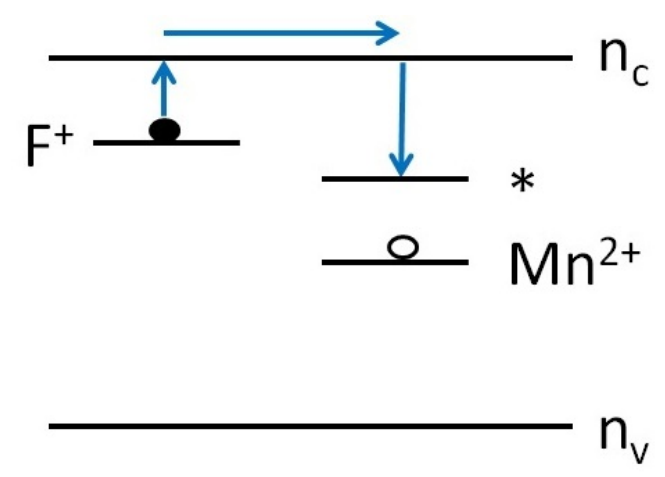

(d)

Figure 5.9: (a) TL for the $\mathrm{NaMgF}_{3}$ sample doped with $0.5 \% \mathrm{Mn}$ (solid curve). The TL data was detected with the PMT after 1 min X-ray irradiation $(40 \mathrm{kV}, 40 \mathrm{~mA})$ at a heating rate of $1 \mathrm{~K} / \mathrm{s}$. The fitted peaks were obtained using first order kinetics glow curve deconvolution (dashed curves).

(b) TL spectra vs. wavelength for the different temperatures detected after 5 min of X-ray irradiation $(40 \mathrm{kV}, 40 \mathrm{~mA})$ detected with the CCD.

(c) Normalized TL intensity of the TL curves at $443 \mathrm{~K}$ (blue curve) and $622 \mathrm{~K}$ (black curve).

(d) Model for F-centre recombination close to the regular $\mathrm{Mn}^{2+}$ site. 


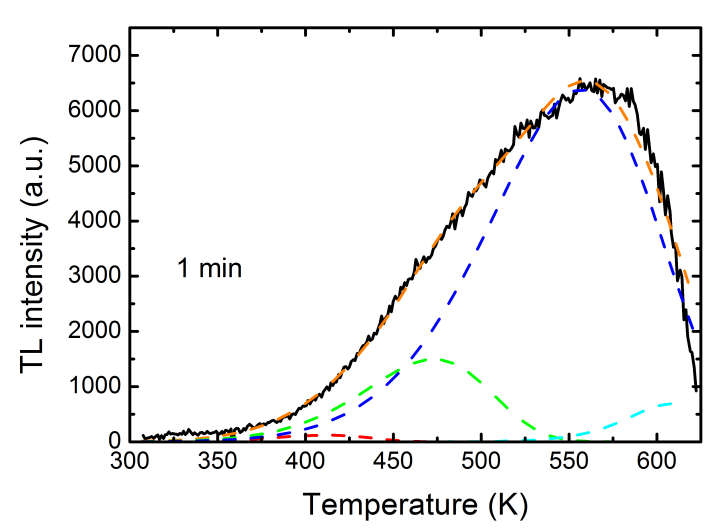

(a)

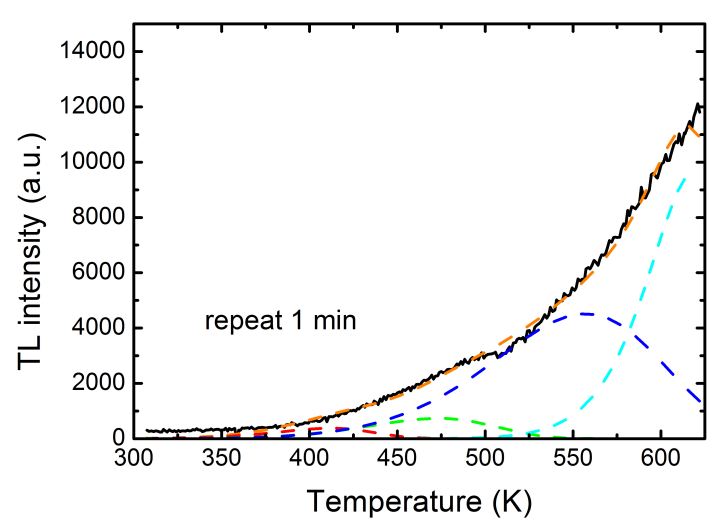

(c)

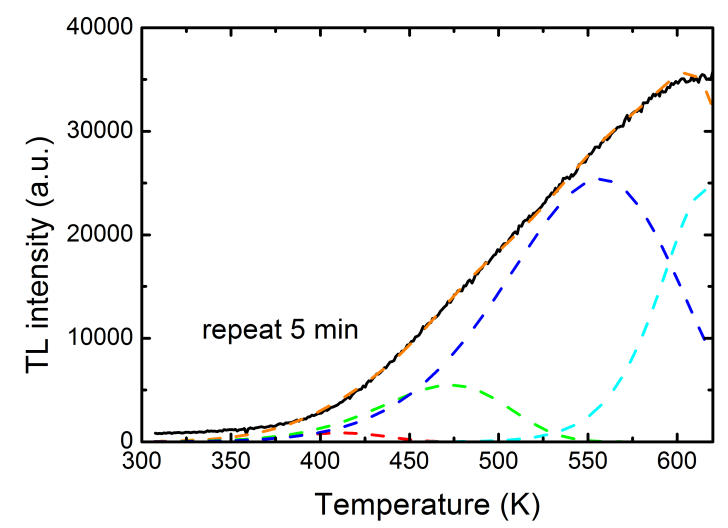

(b)

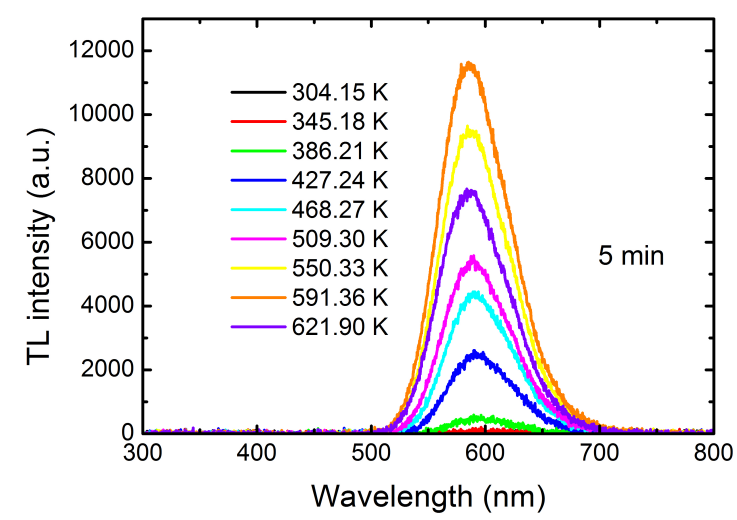

(d)

Figure 5.10: TL for the $\mathrm{NaMgF}_{3}$ sample doped with $1 \% \mathrm{Mn}$ (solid curve). The TL data was detected with the PMT after 1 min (a), repeated after another 5 min (b) and repeated again after $1 \mathrm{~min}(\mathrm{c})$ of $\mathrm{X}$-ray irradiation $(40 \mathrm{kV}, 40 \mathrm{~mA})$ at a heating rate of $1 \mathrm{~K} / \mathrm{s}$. The fitted peaks were obtained using first order kinetics glow curve deconvolution (dashed curves).

(d) TL spectra vs. wavelength for the different temperatures detected after 5 min of X-ray irradiation $(40 \mathrm{kV}, 40 \mathrm{~mA})$ detected with the CCD.

An irradiation time of 1 minute, 5 minutes and again 1 minute was used for TL measurements of the $1 \% \mathrm{Mn}$ doped sample and using a heating rate of $1 \mathrm{~K} / \mathrm{s}$ when the data was collected using a PMT (Fig. 5.10) (a-d). This was performed to observe the dependence 
of the TL signal on an initial dose. The TL after 5 min irradiation was collected using a PMT as well as a CCD (Fig. 5.10 (b+d)). The TL emission mainly arises from the $\mathrm{Mn}^{2+}$ emission at $600 \mathrm{~nm}$ (Fig. $5.10(\mathrm{~d})$ ). There is no TL emission detected from the $500 \mathrm{~nm}$ emission. The peaks of the glow curves were all fitted with first order kinetics glow curve deconvolution for the different samples and doses but this will only be shown for the TL after 5 min of irradiation (see Fig $5.10(b)$ ).

Fig. 5.11(a) shows that the TL intensity is dependent on dose and the history of the initial dose. The sample was irradiated with a dose of 96 Gy and TL was measured. After this, the sample was irradiated again with a dose of 228 Gy and TL was measured again. Then, the sample was irradiated again with a dose of 96 Gy, which leads to a total dose of 420 Gy. TL measurements were performed again. The TL glow curve shows a different shape after receiving a dose of 96 Gy compared to receiving a total dose of 420 Gy. This might be due to the probability of filling traps at a higher temperature is more likely after receiving a higher dose. The shape of the curve above $550 \mathrm{~K}$ seems broader, and if fitting the TL glow curve with a minimum number of peaks with the first order kinetics glow curve deconvolution, the peak above $600 \mathrm{~K}$ shifts towards higher temperatures. But this might just be due to the fact that this peak could also be fitted with two peaks: one at $608 \mathrm{~K}$ and one at higher temperature, which result in the filling of additional traps after receiving a higher dose.

Fig. 5.11(b) shows the shift of the maximum of the TL emission with increasing temperature of the $1 \% \mathrm{Mn}$ doped sample. The shift is from $593 \mathrm{~nm}$ to $586 \mathrm{~nm}$ for the $1 \%$ sample. The glow curve is also broader for higher temperatures. The effect is similar to the temperature dependence of the band gap in semiconductors where the amplitude of atomic vibrations increases leading to larger spacing between atoms with increasing temperature [87, 88, 89, 90]. 


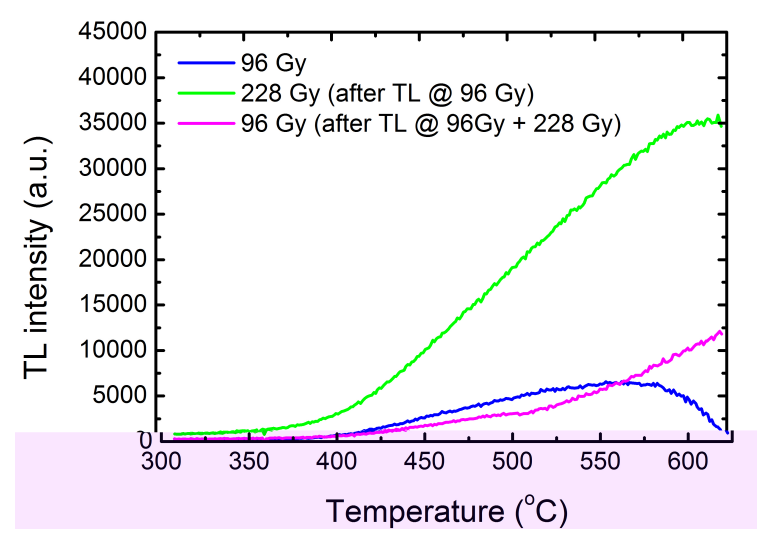

(a)

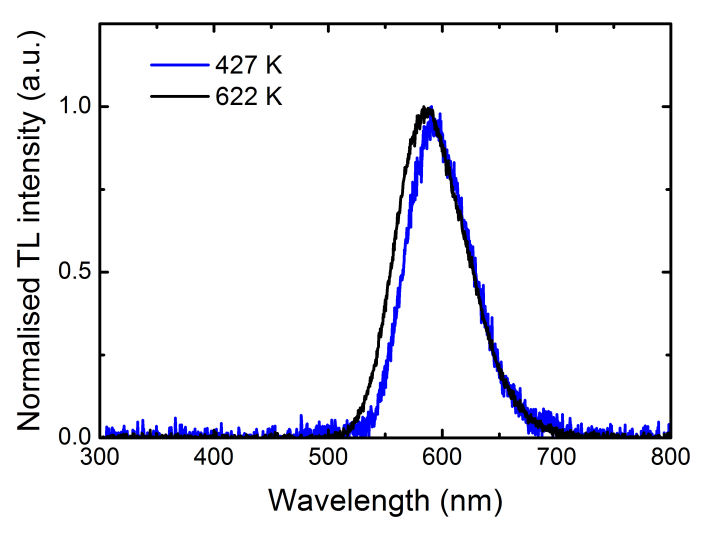

(b)

Figure 5.11: (a) TL for the $\mathrm{NaMgF}_{3}$ sample doped with $1 \% \mathrm{Mn}$ (solid curve). The TL data was detected with the PMT after $1 \mathrm{~min}, 5 \mathrm{~min}$ and again $1 \mathrm{~min}$ X-ray irradiation.

(b) Normalized TL intensity of the TL curves at $427 \mathrm{~K}$ (blue curve) and 622 K (black curve).

All of the glow curves have a low temperature peak at $412 \mathrm{~K}$ and an activation energy of $0.5 \mathrm{eV}$ was calculated. An activation energy of $0.5 \mathrm{eV}$ was calculated for the second peak at $473 \mathrm{~K}$ for the $1 \%$ doped samples and at $464 \mathrm{~K}$ for the $0.5 \%$ doped sample. This peak shifts to lower temperatures for the $0.5 \% \mathrm{Mn}$ doped sample. The temperature for the $3^{\text {rd }}$ peak was measured at $\sim 525 \mathrm{~K}$ for the $0.5 \% \mathrm{Mn}$ and at $\sim 557 \mathrm{~K}$ for the $1 \% \mathrm{Mn}$ doped sample with an activation energy of $0.51 \mathrm{eV}$. The $4^{\text {th }}$ peak shifts from $608 \mathrm{~K}$ for the 1 $\%$ doped sample without any initial dose to $618 \mathrm{~K}$ for the highest initial dose and shows an activation energy of $1.42 \mathrm{eV}$. This peak shift might be a result of radiation damages in the material which induces deeper defects. The higher the initial dose, the higher the temperature for the $4^{\text {th }}$ peak of the glow curve becomes. For the $0.5 \%$ doped sample, the temperature of the $4^{\text {th }}$ peak was around $619 \mathrm{~K}$. 
Table 5.6: The activation energies of the individual glow curve peaks for $\mathrm{NaMgF}_{3}$ doped with different concentrations of Mn obtained by first order kinetics glow curve deconvolution. The top line is the activation energy for the different peaks for the glow curves.

\begin{tabular}{|l|l|l|l|l|}
\hline & $\begin{array}{l}0.50 \pm 0.05 \\
\mathrm{eV}\end{array}$ & $\begin{array}{l}0.50 \pm 0.05 \\
\mathrm{eV}\end{array}$ & $\begin{array}{l}0.51 \pm 0.05 \\
\mathrm{eV}\end{array}$ & $\begin{array}{l}1.42 \pm 0.14 \\
\mathrm{eV}\end{array}$ \\
\hline \hline $\begin{array}{l}0.5 \%(1 \mathrm{~min}) \\
\text { peak height }\end{array}$ & $\begin{array}{l}41 \mathrm{~K} \\
500\end{array}$ & $\begin{array}{l}464 \mathrm{~K} \\
2300\end{array}$ & $\begin{array}{l}580 \mathrm{~K} \\
15000\end{array}$ & $\begin{array}{l}619 \mathrm{~K} \\
3700\end{array}$ \\
\hline $\begin{array}{l}1 \%(1 \mathrm{~min}) \\
\text { peak height }\end{array}$ & $412 \mathrm{~K}$ & $473 \mathrm{~K}$ & $557 \mathrm{~K}$ & $608 \mathrm{~K}$ \\
\hline $1 \%$ (repeat 5 min) & 120 & 1500 & 6400 & 700 \\
peak height & $412 \mathrm{~K}$ & $4730 \mathrm{~K}$ & $557 \mathrm{~K}$ & $616 \mathrm{~K}$ \\
\hline $1 \%$ (repeat 1 min) & 900 & 5500 & 25000 & 24000 \\
\hline peak height & $412 \mathrm{~K}$ & $473 \mathrm{~K}$ & $557 \mathrm{~K}$ & $619 \mathrm{~K}$ \\
\hline
\end{tabular}

These values compare well with the values obtained for the $\mathrm{NaMgF}_{3}$ samples doped with Eu. It can be assumed that this peak is from the same defect since the activation energy and peak temperatures are similar [87, 88, 89, 90].

Dotzler reported in his thesis that the TL only arises from the $600 \mathrm{~nm}$ emission, and additional peaks at low temperature $(473 \mathrm{~K})$ were observed. The main peak is around 573 $\mathrm{K}$ in these measurements. It is not clear where this low temperature peak is coming from, and it seems to disappear with increasing Mn concentration $(>0.2 \%)$. The samples that were used in this thesis had Mn concentrations higher than $0.2 \%$, and this could be the reason for the absence of the low temperature peak around $474 \mathrm{~K}$. Dotzler also reported the existence of PTTL (phototransferred thermoluminescence), which he has observed with the $0.02 \% \mathrm{Mn}$ doped sample [3]. These measurements were also performed: the $1 \%$ doped sample which was irradiated for $1 \mathrm{~min}$ and bleached for $2 \mathrm{~s}$ with a $405 \mathrm{~nm}$ laser, using a 
$0.3 \%$ neutral density filter (Fig. 5.12). There was no PTTL observed and the increase above $500 \mathrm{~K}$ comes from the black body radiation. Dotzler used also a different laser with less power, and it might be that the power of the laser used in this thesis is too high for the proof of PTTL [3].

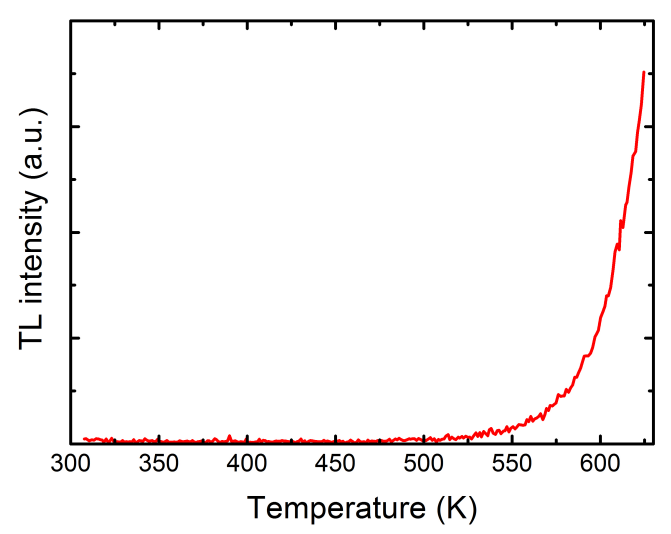

Figure 5.12: TL for $\mathrm{NaMgF}_{3}: 1 \% \mathrm{Mn}$ after 1 min irradiation and $2 \mathrm{~s}$ of bleaching with a $405 \mathrm{~nm}$ laser using a $0.3 \%$ neutral density filter detected with the PMT.

\subsection{Radioluminescence and temperature dependent RL measurements}

Long-term radioluminescence measurements and temperature dependent RL measurements were performed with the X-ray generator $(40 \mathrm{kV}, 40 \mathrm{~mA})$ using an Aluminium filter. For the temperature dependent RL measurements, the samples were powdered, immersed in isopropanol and dispersed on a resistor to control heating for different temperatures up to $100{ }^{\circ} \mathrm{C}$. At a certain temperatures, the samples were also thermally bleached. 


\subsubsection{Radioluminescence measurements}

\subsubsection{Eu doped polycrystalline $\mathrm{NaMgF}_{3}$}

Fig. 5.13 shows the results for the RL measurements on $0.2 \% \mathrm{Eu}$ doped $\mathrm{NaMgF}_{3}$. The peaks in the RL spectrum for the $\mathrm{Eu}$ sample can be attributed to the $\mathrm{Eu}^{2+}$ and $\mathrm{Eu}^{3+}$ transitions. The emission from $360 \mathrm{~nm}$ to $420 \mathrm{~nm}$ arises from $\mathrm{Eu}^{2+}$ ion and from $590 \mathrm{~nm}$ to $715 \mathrm{~nm}$ from the $\mathrm{Eu}^{3+}$ ion. From $\sim 400 \mathrm{~nm}$ to $580 \mathrm{~nm}$, a broad peak is detected at low doses and disappears for high doses (Fig. 5.13(a)). The emission wavelengths in the RL spectrum of $\mathrm{Eu}^{2+}$ and $\mathrm{Eu}^{3+}$ did not change after irradiation.

The RL spectrum as a function of dose in Fig. 5.13(b) shows that the integrated intensity $\left(\mathrm{I}_{R L}\right)$ of the $\mathrm{Eu}^{2+}$ ion increases by $50.5 \%$ and reaches a maximum after a dose of 850 Gy. After this initial increase the $\mathrm{I}_{R L}$ intensity starts to decrease. After a dose of $13 \mathrm{kGy}$ it has been decreased by $48.5 \%$ which is $22.5 \%$ smaller than the starting value. The low RL signal until 850 Gy is caused by the existence of additional electron-hole-traps next to the $\mathrm{Eu}^{2+}$, see Fig. 5.13(c). Electron and holes, which are mobile after irradiation, will more likely recombine on the electron and hole traps and therefore, less electrons get trapped in the $\mathrm{Eu}^{2+}$. This results in a low RL signal of $\mathrm{Eu}^{2+}$ until $850 \mathrm{~Gy}$. The electron and hole traps saturate after higher irradiation and hence, the RL signal of the $\mathrm{Eu}^{2+}$ increases after receiving a dose of 850 Gy. Above $\sim 1 \mathrm{kGy}$, the RL signal of the $\mathrm{Eu}^{2+}$ decreases (Fig. $5.13(\mathrm{~b})$ ), which can be due to the creation of non-radiative defects and/or the conversion of $\mathrm{Eu}^{2+} \rightarrow \mathrm{Eu}^{3+}$. Fig. 5.13(d) shows that radiation generates a non-radiative recombination centre next to $\mathrm{Eu}$. This non-radiative centre competes with $\mathrm{Eu}$ and the probability for mobile carriers to recombine in this centre is higher compared to Eu. Therefore, the RL signal for Eu decreases for doses above $\sim 1 \mathrm{kGy}$. 


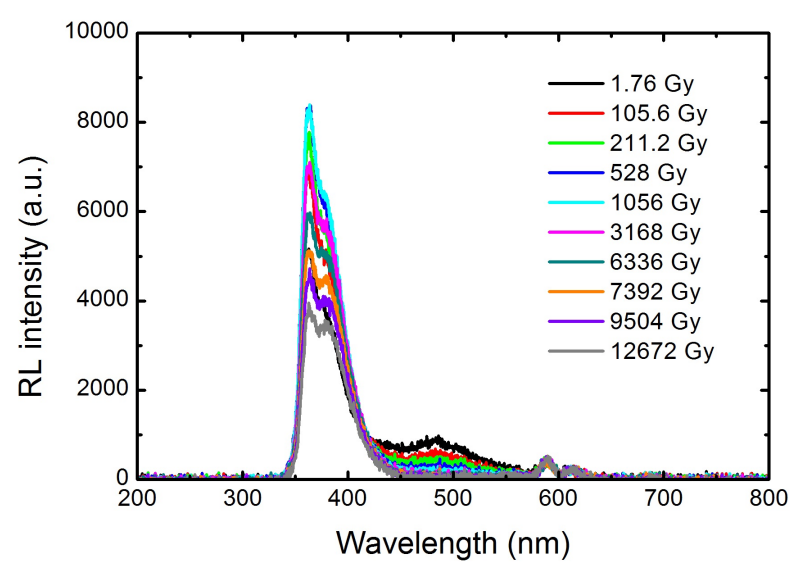

(a)

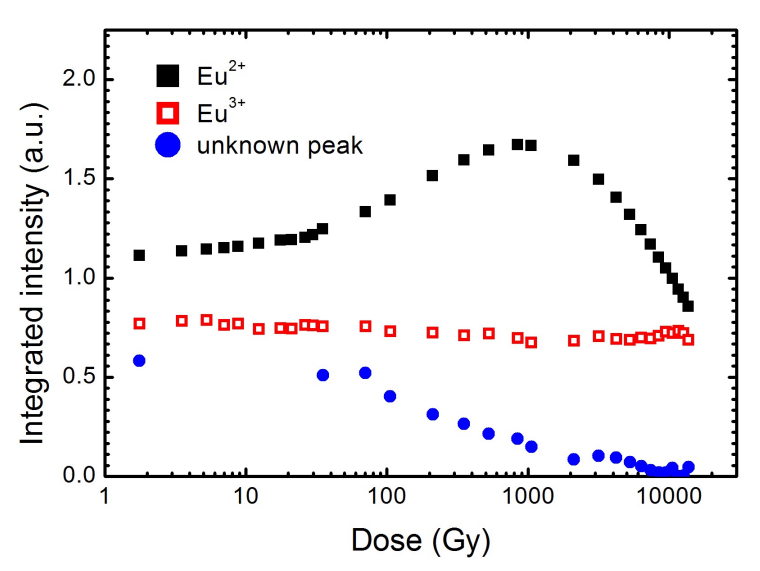

(b)
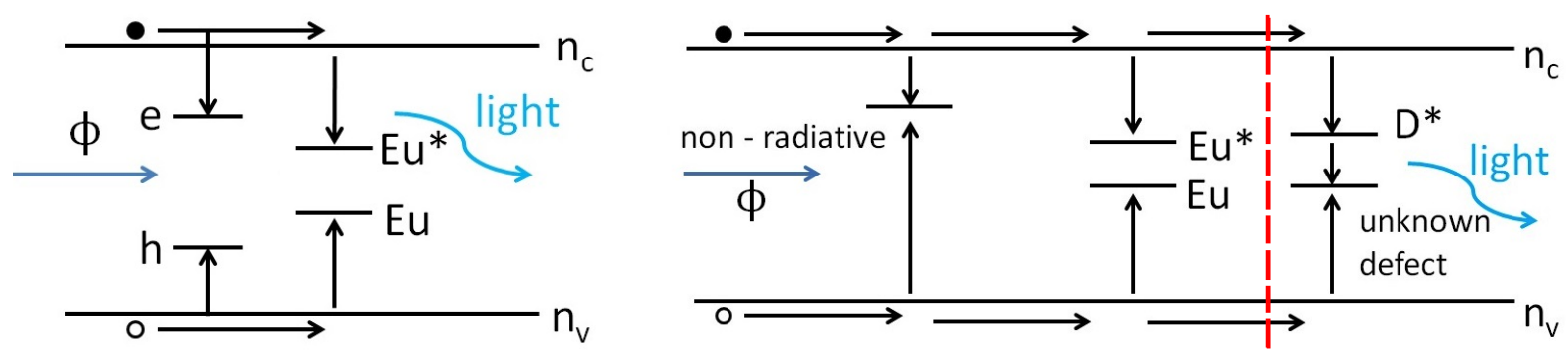

(c)

(d)

Figure 5.13: (a) Radioluminescence spectra for $\mathrm{NaMgF}_{3}: 0.2 \% \mathrm{Eu}$ for different doses and (b) the integrated RL spectrum as a function of dose during continuous X-ray irradiation $\left(\mathrm{Eu}^{2+}\right.$ filled squares and $\mathrm{Eu}^{3+}$ open squares).

(c) Existence of electron and hole traps next to the Eu.

(d) Dose dependence of the RL signal as a result of the generation of nonradiative recombination centres.

$\mathrm{I}_{R L}$ for the $\mathrm{Eu}^{3+}$ signal is nearly independent of dose as shown in Fig. 5.13(b). This is a desirable property for a real-time dosimeter that can detect low doses as well as high doses without being damaged. The ratio of ${ }^{7} \mathrm{~F}_{2}:{ }^{7} \mathrm{~F}_{1}$ changes from $2: 1$ before irradiation to $1: 1$ after heavy irradiation in the PL spectra, and in the RL spectrum a change from 1.8:1 at 1.8 Gy and 1.4:1 at $12 \mathrm{kGy}$ was observed. This means that the average crystal environment 
of the $\mathrm{Eu}^{3+}$ ion seems less distorted after heavy irradiation with a dose of $12 \mathrm{kGy}$. The PL measurements were performed for a higher dose $(19 \mathrm{kGy})$ and therefore, the change of the ratio ${ }^{7} \mathrm{~F}_{2}:{ }^{7} \mathrm{~F}_{1}$ is bigger. The $\mathrm{RL}$ signal of the $\mathrm{Eu}^{2+}$ emission changes with dose, which was also observed in the PL spectra measured before and after heavy irradiation (Fig. 5.3(a)). The ratio of integrated intensity of the $\mathrm{E}_{g}$ transition to the ${ }^{6} \mathrm{P}_{J}$ emission increases from 1.3:1 at 850 Gy to $1.7: 1$ at $\sim 12.7 \mathrm{kGy}$. This is consistent with the PL emission spectra where the ratio changes from 1.4:1 before irradiation to 2:1 after irradiation with a dose of 19 kGy. That means for further irradiation up to $19 \mathrm{kGy}$ the intensity of the emission of the unknown defect centred at $380 \mathrm{~nm}$ increases due to the creation of defects next to the $\mathrm{Eu}^{2+}$ ion, which results in a decrease of the energy level between the $5 \mathrm{~d}$ and $4 \mathrm{f}$ level and increases the transition from the $5 \mathrm{~d}$ level on top of the overall decrease of the $\mathrm{Eu}^{2+}$ emission.

The RL intensity is initially low because some of the X-ray generated electrons and holes become trapped and hence electrons and holes available for RL are low. For a high enough dose, all of the traps are filled and hence all of the X-ray generated electrons and holes are available for RL. Thus, the RL will increase with dose until all of the electron and hole traps are filled. This explains the initial increase in the RL (Fig. 5.13(c)). The RL starts to decrease at high doses due to X-ray induced radiation damage that results in non-radiative recombination sites. Thus, as shown in Fig. 5.13(d), some of the X-ray generated electrons and holes are not available for RL due to non-radiative recombination, which leads to a reduction in the $\mathrm{RL}$ for high doses.

The RL emission wavelength of the broad unknown defect detected from $450 \mathrm{~nm}$ until 580 $\mathrm{nm}$ is the same as observed for the PL emission spectrum (Fig. 5.13(a)). The integrated RL intensity of the unknown defect decreases with increasing dose. This might be due to the fact that the probability for the mobile charges to recombine in a non-radiative recombination site is very high and therefore, there is no RL detected of the unknown defect (Fig. 5.13(d)). 


\subsubsection{2 $\mathrm{Mn}$ doped polycrystalline $\mathrm{NaMgF}_{3}$}

The RL spectrum for both Mn concentrations can be completely attributed to the regular and highly distorted $\mathrm{Mn}^{2+}$ site. The peak at $500 \mathrm{~nm}$ arises from the highly distorted site and at $600 \mathrm{~nm}$ from the regular site. There is no shift in the RL emission wavelengths after heavy irradiation (Fig. 5.14(a-d)). The RL spectrum and the integrated intensity for 0.5 \% Mn doped $\mathrm{NaMgF}_{3}$ are plotted in Fig. 5.14(a+b). Fig. 5.14(b) shows $\mathrm{I}_{R L}$ versus the dose for the $0.5 \%$ sample and the RL signal for the regular $\mathrm{Mn}^{2+}$ increases by $259.7 \%$ and reaches a maximum after a dose of $8.5 \mathrm{kGy}$. After this initial increase the RL intensity starts to decrease. After a dose of $11.6 \mathrm{kGy} \mathrm{I}_{R L}$ has been decreased by $2.2 \%$, which is $251.6 \%$ higher than the starting dose. For the distorted site, $\mathrm{I}_{R L}$ increases by $40 \%$ and reaches a maximum after a dose of $5.3 \mathrm{kGy}$. After this initial increase the RL intensity starts to decrease. After a dose of $12.7 \mathrm{kGy}_{R L}$ has been decreased by $42.9 \%$, which is 20 $\%$ less than the value at $\sim 1.8$ Gy. The ratio of the peaks of regular to the highly distorted $\mathrm{Mn}^{2+}$ site changes from 18:1 at 1.8 Gy to 70:1 at 8.5 kGy.

$\mathrm{I}_{R L}$ for the regular Mn site for the $1 \%$ doped sample increases by $403 \%$ and reaches a maximum after a dose of $8.5 \mathrm{kGy}$. After this initial increase the RL intensity starts to decrease. After a dose of $12 \mathrm{kGy}$ it has been decreased by $0.36 \%$. The RL of the highly distorted site increases by $140 \%$ until $5.3 \mathrm{kGy}$. After this increase $\mathrm{I}_{R L}$ decreases by $9.4 \%$ until $12 \mathrm{kGy}$, which is $118.2 \%$ of the starting value. The ratio of the peaks of regular to the highly distorted $\mathrm{Mn}^{2+}$ change from 18:1 at 1.8 Gy to 70:1 at $8.5 \mathrm{kGy}$. For the $0.5 \%$ Mn doped sample the ratio of the regular $\mathrm{Mn}^{2+}$ to the distorted $\mathrm{Mn}^{2+}$ site was nearly 1:1 in the PL and therefore, a higher ratio of the regular site to the distorted site was expected to be observed in the RL spectra. 


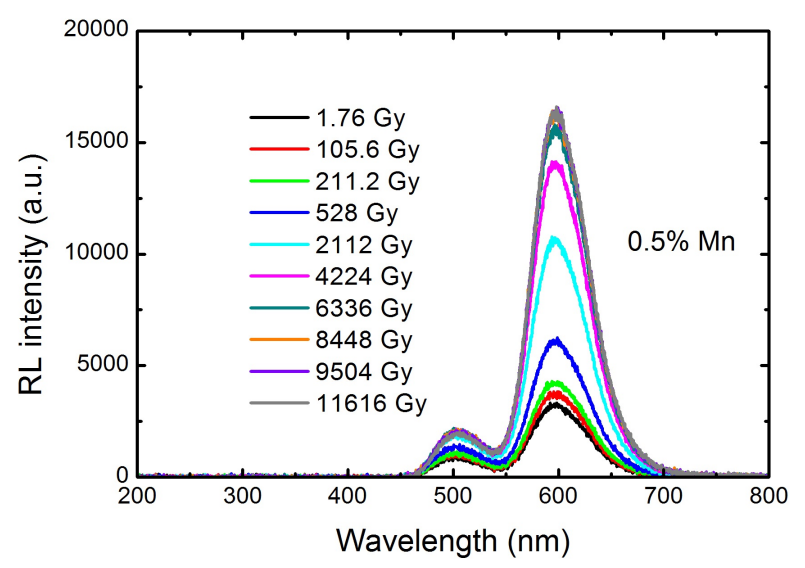

(a)

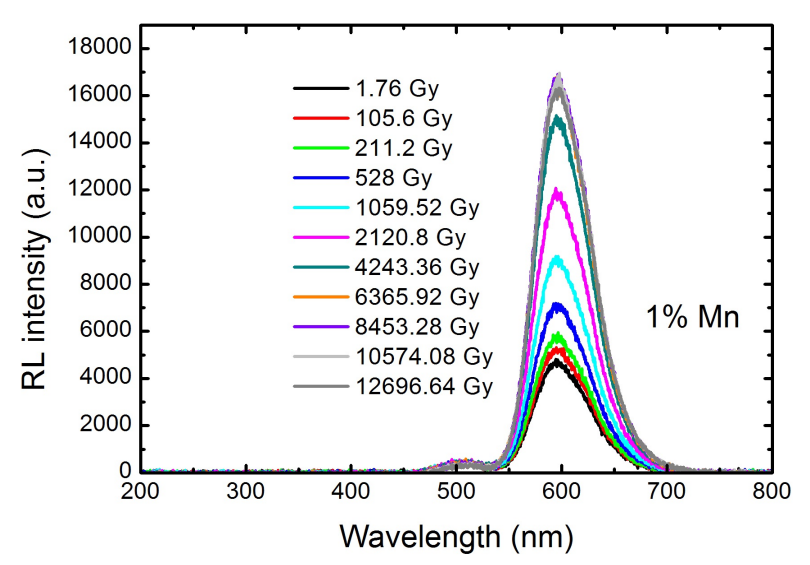

(c)

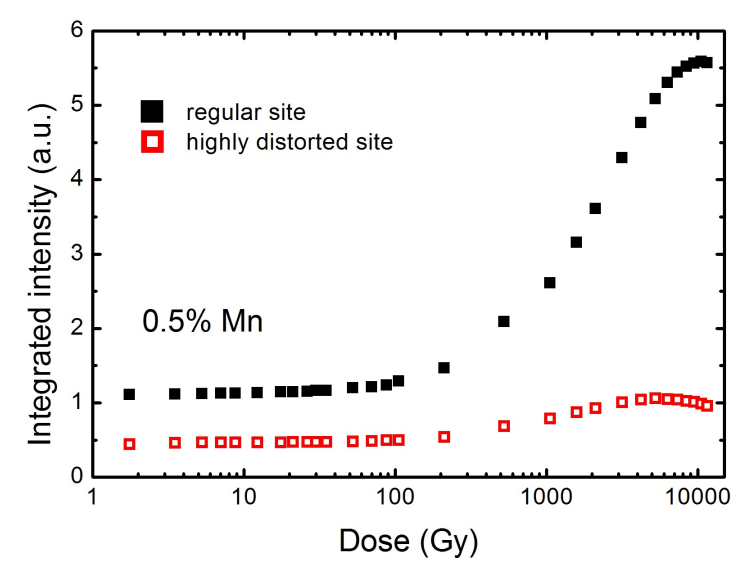

(b)

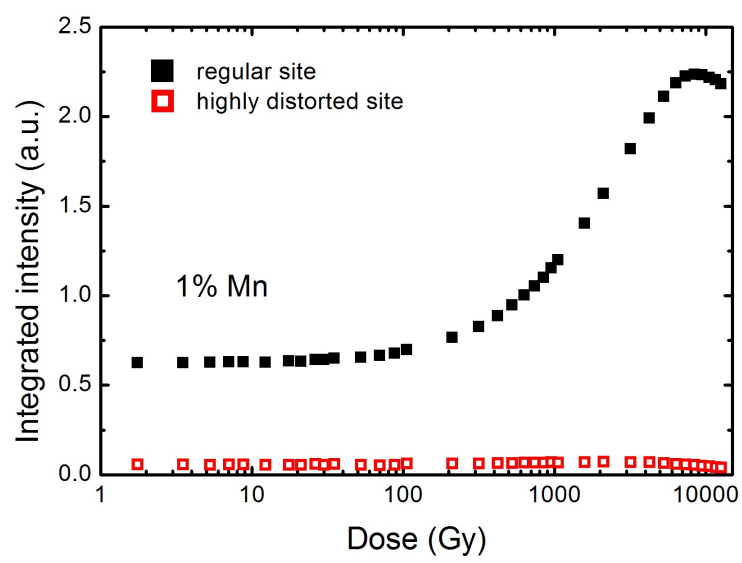

(d)

Figure 5.14: (a) Radioluminescence spectra for $\mathrm{NaMgF}_{3}: 0.5 \% \mathrm{Mn}$ for different doses and (b) the integrated RL spectra as a function of dose during continuous X-ray irradiation (regular $\mathrm{Mn}^{2+}$ site filled squares and highly distorted $\mathrm{Mn}^{2+}$ site open squares).

(c) Radioluminescence spectra for $\mathrm{NaMgF}_{3}: 1 \% \mathrm{Mn}$ for different doses and

(d) the integrated RL spectra as a function of dose during continuous X-ray irradiation (regular $\mathrm{Mn}^{2+}$ site filled squares and highly distorted $\mathrm{Mn}^{2+}$ site open squares). 


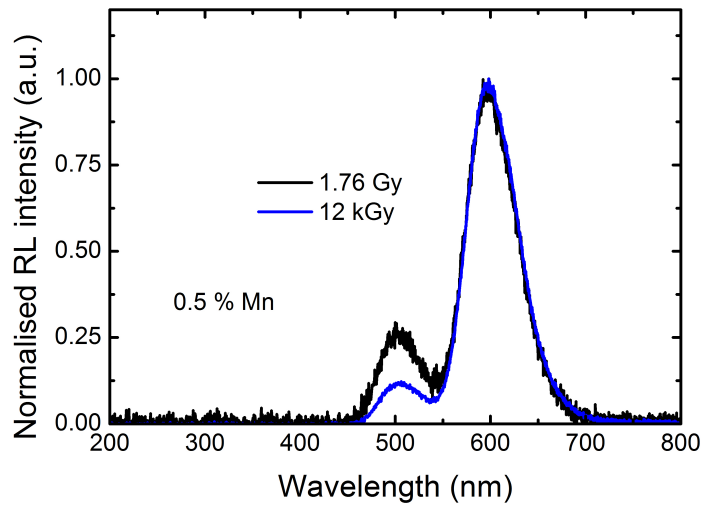

(a)

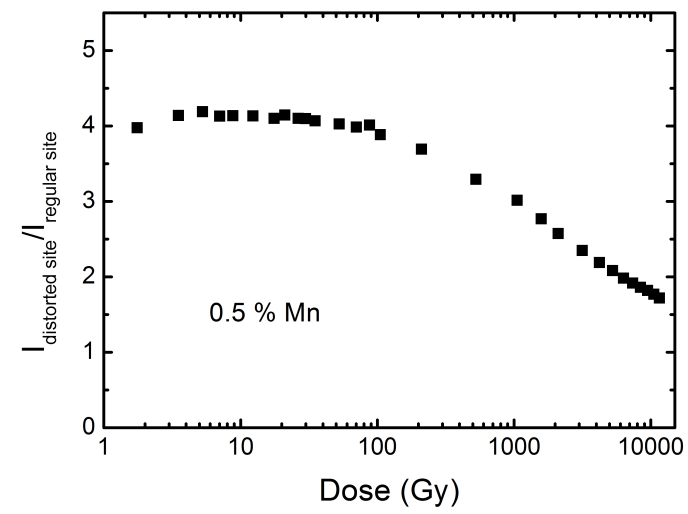

(c)

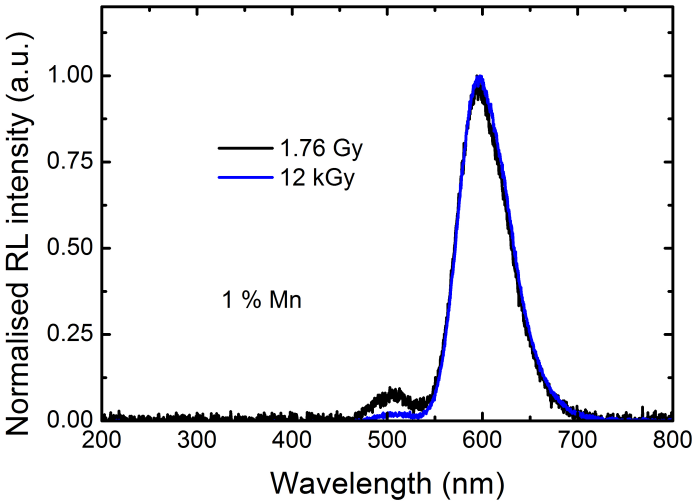

(b)

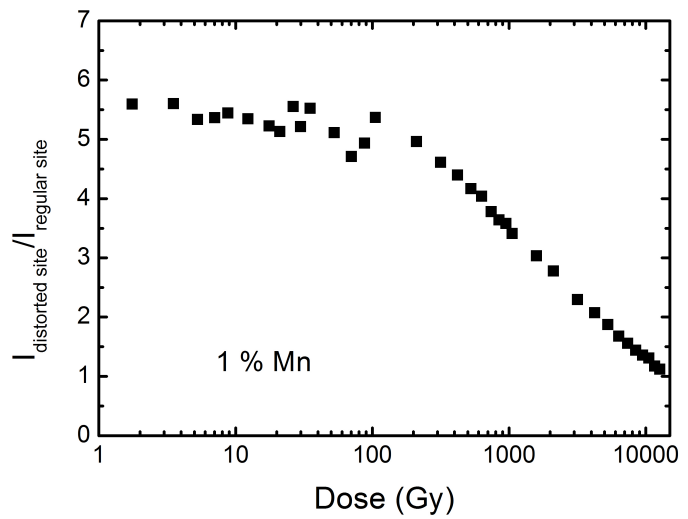

(d)

Figure 5.15: Normalised RL spectra for low doses (blue curve) and after 12 kGy irradiation (black curve) for $0.5 \%$ (a) and $1 \% \mathrm{Mn}$ (b) doped sample.

Ratio of the RL intensity of the distorted peak divided by the RL intensity of the regular peak as a function of dose for $0.5 \% \mathrm{Mn}(\mathrm{c})$ and $1 \% \mathrm{Mn}(\mathrm{d})$ doped $\mathrm{NaMgF}_{3}$.

The normalised RL curve for low radiation and after heavy irradiation shows that the intensity of the distorted site decreases with increasing dose for both concentrations of the Mn doped samples (Fig. 5.15 $(\mathrm{a}+\mathrm{b})$ ). This is due to the probability for filling the distorted traps being lower than filling the traps from the regular $\mathrm{Mn}^{2+}$ site. In Fig. $5.15(\mathrm{c}+\mathrm{d})$ the ratio of the RL intensity of the distorted peak to the regular peak is plotted against the 
dose. This ratio is constant up to 100 Gy. After that it decreases for doses until 12.7 kGy. The decrease might be due to the generation of non - radiative defects near the distorted site.

\subsubsection{Temperature dependent RL measurements}

The temperature dependent measurements were performed by putting the powdered samples immersed with isopropanol on a ceramic holder and heating the holder up until a certain temperature. After the temperature had stabilized RL measurements up to a dose of 15 Gy were performed. Then the sample was thermally bleached by heating up it until $350{ }^{\circ} \mathrm{C}$. This process was repeated for 13 different temperature settings. The total dose the sample has received at the end was $\sim 200$ Gy.

\subsubsection{Eu doped polycrystalline $\mathrm{NaMgF}_{3}$}

The temperature dependent RL signal increases by $6.2 \%$ compared to room temperature when the temperature is increased to $100{ }^{\circ} \mathrm{C}$ for the $\mathrm{NaMgF}_{3}: 0.2 \% \mathrm{Eu}$ doped sample and the RL intensity increasesby $5.2 \%$ compared to $100{ }^{\circ} \mathrm{C}$ when the temperature is decreased to $40{ }^{\circ} \mathrm{C}$ (Fig. 5.16). The RL signal for different temperature steps shows a non-linear behaviour. This might be due to the noise from the system. At low temperature, the RL signal is lower due to electron-hole-trapping overlapping the RL signal. This was already explained for the shape of the RL signal at low doses (Fig. 5.13(c)). The RL is low at room temperature due to some of the mobile carriers being trapped. The probability of thermal detrapping increases with increasing temperature. This means that a smaller fraction of the mobile electrons and holes become trapped as the temperature increases. This results in an increase in the RL with increasing temperature (Fig. 5.16(c)). For small doses and hence a negligible trapped carrier concentration, the RL should then decrease with decreasing temperature. This is not observed. It is likely that the dose at each temperature results in significant carrier trapping. Thus, as the temperature is reduced there will be a 
competition between reducing the RL through reducing the thermal detrapping probability and increasing the RL due to significant trap filling at each temperature. It is apparent in Fig. 5.16(a) that the second process dominates. The thermal coefficient was estimated using the average of the temperature dependent RL curve and was around $0.11 \pm 0.08$ $\% / \mathrm{K}$.

The shape of the $\mathrm{Eu}^{2+} \mathrm{RL}$ emission also changes with increasing temperature. The intensity of the sharp peak centred at $360 \mathrm{~nm}$ decreases compared to room temperature when the temperature is increased to $100{ }^{\circ} \mathrm{C}$, which leads to a higher emission from the $5 \mathrm{~d}$ level. An emission from the $4 \mathrm{f}$ and the $5 \mathrm{~d}$ level is expected. With increasing temperature, the crystal field of the material changes, the energy difference between the $4 \mathrm{f}$ and $5 \mathrm{~d}$ level, $\Delta$, decreases with increasing temperature, making a transition from the $4 \mathrm{f}$ to the $5 \mathrm{~d}$ level more likely. Prompt transition from the $5 \mathrm{~d}$ to the ground state occurs, increasing the RL signal of the broad peak around $380 \mathrm{~nm}$. At low temperatures, traps are only filled through process of radioluminescence. Above a certain temperature, additional deep traps will be emptied. The probability of detrapping these deep traps increases until it becomes the main process. This can be estimated from the following equation,

$$
\tau_{d t}=\tau_{o} e^{-\frac{\Delta_{1}}{k T}}
$$

where $\Delta_{1}$ is the energy of the trap, $\mathrm{k}$ the Boltzmann's constant and T the temperature. Therefore, the RL signal increases with increasing temperature, which is schematically shown in Fig. 5.16(c). 


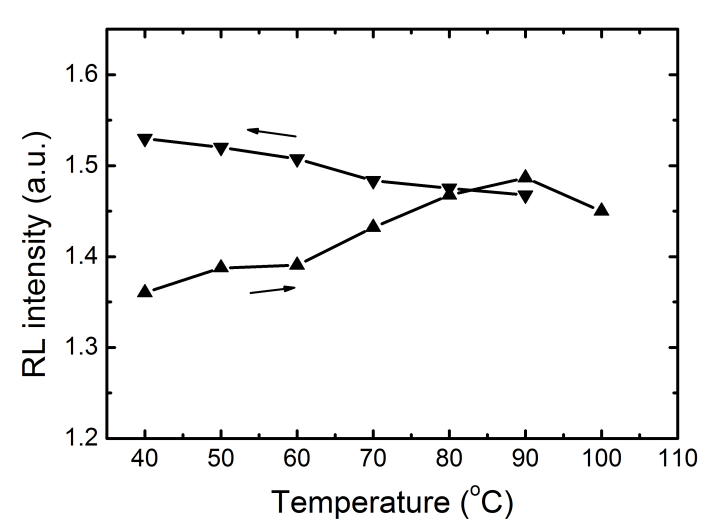

(a)

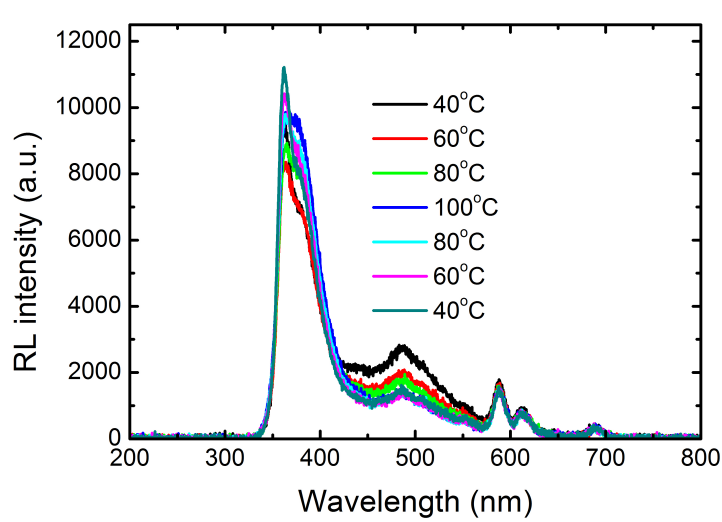

(b)

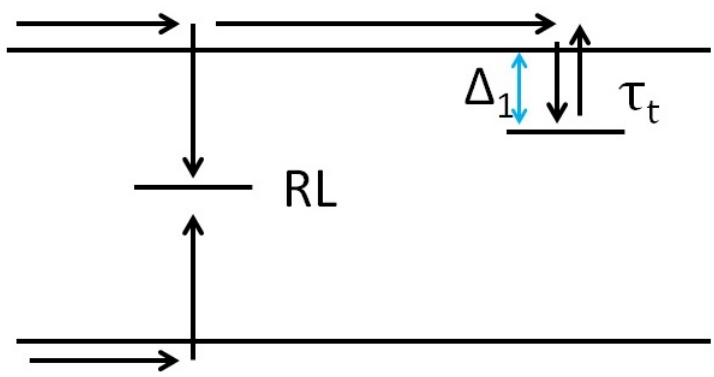

(c)

Figure 5.16: (a) Temperature dependent RL for $\mathrm{NaMgF}_{3}$ : $0.2 \%$ Eu for different temperature up to $100{ }^{\circ} \mathrm{C}$. Arrows facing up signal an increase of temperature, and arrows facing down signal a decrease in temperature.

(b) RL spectra measured for different temperatures.

(c) Model of the RL and TL trapping and detrapping in $\mathrm{NaMgF}_{3}$ doped with $\mathrm{Eu}$.

\subsubsection{2 $\mathrm{Mn}$ doped polycrystalline $\mathrm{NaMgF}_{3}$}

For the $\mathrm{NaMgF}_{3}$ doped with different concentration of $\mathrm{Mn}^{2+}$, the temperature dependent RL intensity was observed (Fig. 5.17). The blue arrows represent the regular $\mathrm{Mn}^{2+}$ site and the red ones represent the distorted $\mathrm{Mn}^{2+}$ in Fig. 5.17(a+b). In Fig. 5.17(a) the 
temperature dependence of the RL intensity of the $0.5 \%$ sample is shown. It was observed that the RL signal shows negligible hysteresis within the noise. For the highly distorted site, it was observed that the RL is not changing with increasing or decreasing temperature. For the regular site, the RL signal is dependent on the temperature. The observation of the regular Mn site is similar to the Eu doped samples (Fig. 5.16(c)).

The $1 \%$ doped sample shows a temperature dependent RL signal. This might be due to the creation of non-radiative recombination centres next to the distorted $\mathrm{Mn}^{2+}$ as already explained for the RL signal. The probability of filling these traps increases with increasing temperature and therefore, the overall RL signal of the highly distorted $\mathrm{Mn}^{2+}$ site decreases. Since the probability of filling these traps is temperature dependent, the rate of filling these traps is decreasing with decreasing temperature from $100{ }^{\circ} \mathrm{C}$ to $40{ }^{\circ} \mathrm{C}$. For the regular site, the RL intensity increases by $3.1 \%$ compared to room temperature when the temperature is increased to $100{ }^{\circ} \mathrm{C}$ and decreases by $1.7 \%$ compared to $100{ }^{\circ} \mathrm{C}$ when the temperature is decreased to $40{ }^{\circ} \mathrm{C}$. The increase of the RL signal for higher temperatures can be explained with the same model that was used for the $0.5 \%$ sample above. It was mentioned previously that for low temperatures, additional electron-hole-trapping overlaps the RL trapping and therefore the RL signal is lower for low temperatures. With increasing temperature the thermal excitation is increasing and additional trapping and detrapping occurs which increases the RL signal. The probability of detrapping deep traps also increases with increasing temperature (Fig. 5.16(c)). 


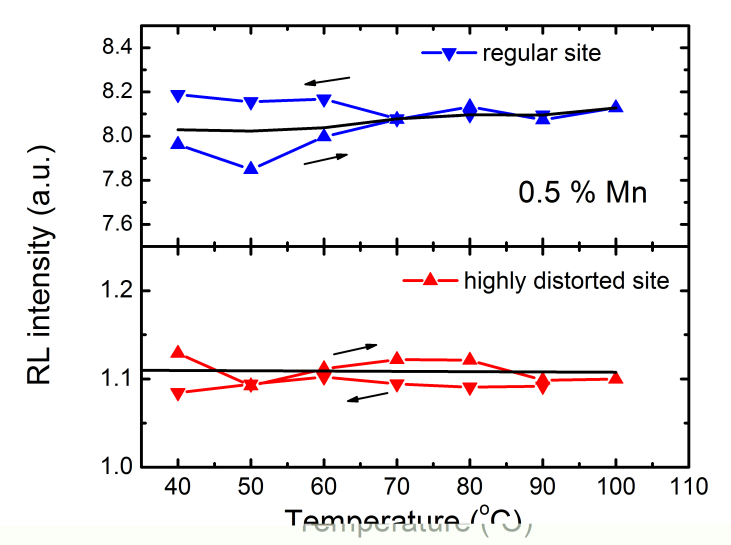

(a)

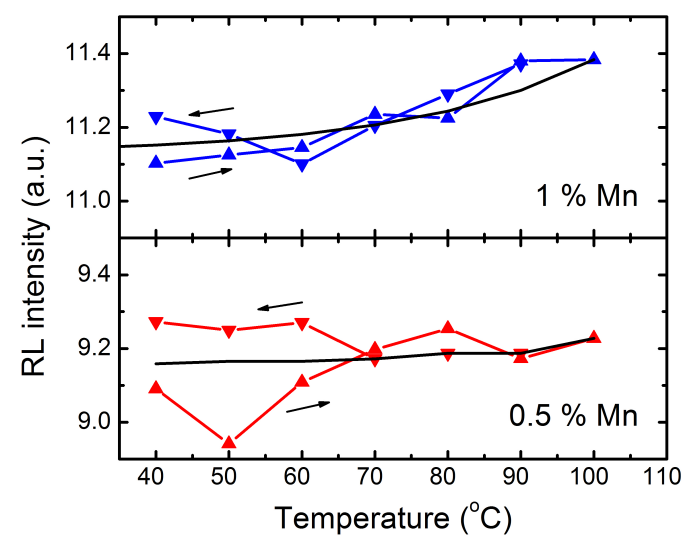

(c)

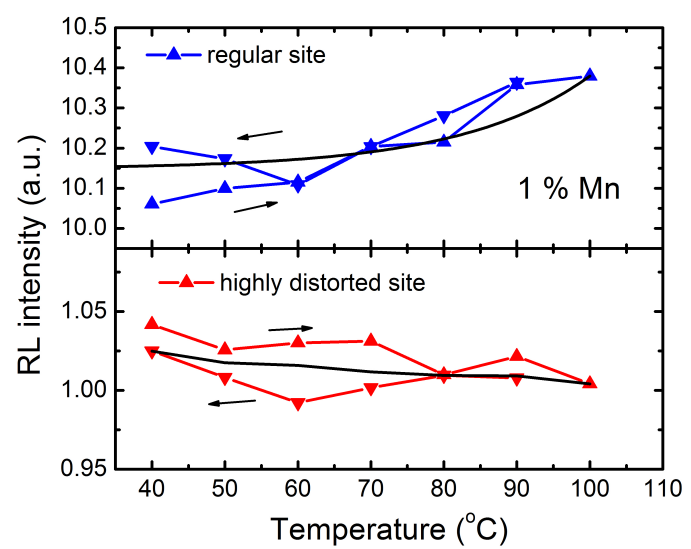

(b)

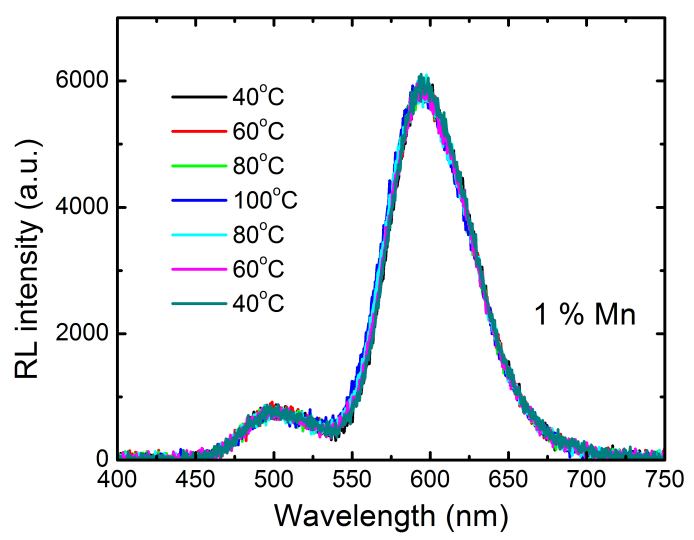

(d)

Figure 5.17: (a) Temperature dependent RL for $0.5 \% \mathrm{Mn}$ and

(b) for $1 \% \mathrm{Mn}$ doped $\mathrm{NaMgF}_{3}$ for different temperature up to $100{ }^{\circ} \mathrm{C}$. Blue indicates the regular $\mathrm{Mn}^{2+}$ site and red the highly distorted site.

(c) Total RL signal of the distorted and the regular site versus the temperature for the $1 \%$ doped sample (top graph) and the $0.5 \%$ sample (bottom graph).

(d) RL spectra measured for different temperatures for $1 \%$ Mn doped $\mathrm{NaMgF}_{3}$.

Arrows facing up signal an increase of temperature, and arrows facing down signal a decrease in temperature. Black curves show the trend of the temperature dependent RL. 
Fig. 5.17(d) shows the RL intensity plotted against the wavelength for different temperatures up to $100{ }^{\circ} \mathrm{C}$. For higher temperatures, the distance between atoms increases which should lead to a change in the emission spectrum. This could result in a shift of the peak towards higher energies or a broadening of the peaks [87, 88, 89, 90]. There was no observable shift in the emission wavelength towards lower wavelengths, in the ratio of the peaks nor in the width of the peaks, which would have been expected because with increasing temperature, the spacing between atoms gets larger with a temperature change from 40 ${ }^{\circ} \mathrm{C}$ to $100{ }^{\circ} \mathrm{C}$. The total temperature dependent signal of both concentrations is plotted in Fig. 5.17(c). The $0.5 \%$ Mn sample shows a nearly temperature independent RL. The variation of the RL signal with increasing or decreasing temperature is more dependent on the RL signal of the regular $\mathrm{Mn}^{2+}$ site because this signal has a higher intensity than the distorted site. Tab. 5.7 summarises the temperature changes for different Mn doped samples. The thermal coefficients of the RL were calculated using the average RL signal for each temperature. The $1 \%$ doped sample shows the biggest dependence of the RL signal on increasing temperature. Whereas the distorted site of the $0.5 \%$ sample shows a nearly independent RL signal with increasing temperature, only the regular site shows an increase of the RL intensity with increasing temperature. All of the thermal coefficients of the RL are smaller than $0.1 \% / \mathrm{K}$, which is a desirable property of a real-time dosimeter.

Table 5.7: Summary of temperature changes of the RL signal for different doped samples and different $\mathrm{Mn}^{2+}$ sites.

\begin{tabular}{|l|l|l|l|}
\hline Concentration $(\%)$ & distorted $(\% / \mathrm{K})$ & regular $(\% / \mathrm{K})$ & total $(\% / \mathrm{K})$ \\
\hline \hline 0.5 & $0 \pm 0.02$ & $0.02 \pm 0.1$ & $0.01 \pm 0.1$ \\
\hline 1 & $0.03 \pm 0.02$ & $0.03 \pm 0.07$ & $0.03 \pm 0.07$ \\
\hline
\end{tabular}




\subsection{Optically stimulated luminescence measurements}

Optically stimulated luminescence (OSL) measurements were only performed for the $\mathrm{Eu}^{2+}$ ions in the $\mathrm{NaMgF}_{3}$ sample. The sample was transported in a light-tight box between all the measurements. The OSL measurements were performed for different doses as shown in Fig. 5.18(a). After each irradiation, the sample was taken out of the X-ray generator and OSL was measured with an emission wavelength of $360 \mathrm{~nm}$ and detected at $410 \mathrm{~nm}$. The irradiation was performed with an X-ray generator operator operating at $40 \mathrm{~mA}$ and $40 \mathrm{kV}$.

The total intensity of the OSL signal was calculated and plotted against the dose in Fig. 5.18(b). The OSL signal rapidly increases until a dose of $\sim 1 \mathrm{kGy}$, and then it increases at a slower rate. The signal will probably saturate at higher doses. This is consistent with the dose dependence of the RL intensity. It was shown that the $\mathrm{I}_{R L}$ is increasing for doses up to $\sim 850 \mathrm{~Gy}$, and then is decreasing up to $12.7 \mathrm{kGy}$ (Fig. 5.13(b)). The decrease of the RL signal for doses above $1 \mathrm{kGy}$ results in a slower increase of the OSL signal for doses up to $19 \mathrm{kGy}$. This result leads to the assumption that the carriers are recombining at the same traps. Fig. 5.18(c) shows the change of the OSL signal ( $\Delta$ OSL) divided by the change of dose versus the total dose. The signal of $\Delta$ OSL/ $\Delta$ dose is nearly constant until $\sim 1 \mathrm{kGy}$ and decreases until $19 \mathrm{kGy}$. This suggests that a degradation effect is occurring at higher doses, leading to a decrease of the $\triangle$ OSL signal. The OSL intensity at around 200 Gy was significantly lower than expected. This might be a result of the method of transportation the sample. During the process of irradiation and measuring the OSL, the sample could have flipped over or could have been accidentally illuminate. Therefore, there was a smaller intensity of the OSL measured. To investigate this hypothesis, measurements were repeated for 211 Gy, 4 kGy and again 211 Gy (Fig. 5.18(d)). The sample was mounted on a transportable holder for photoluminescence measurements and the whole holder was placed in the X-ray generator. After radiation the holder with sample was transported back in the light-tight box to the spectrometer and the OSL measurement was repeated with the sample in the same position. It can be seen that the OSL signal decreases after 
receiving a heavy dose of $4 \mathrm{kGy}$ (and a total dose of $4.2 \mathrm{kGy}$ ). The second measurement after receiving an additional dose of $211 \mathrm{~Gy}$ (total dose of $4.4 \mathrm{kGy}$ ) also shows a decrease in the OSL signal compared to the first measurement after a dose of 211 Gy.

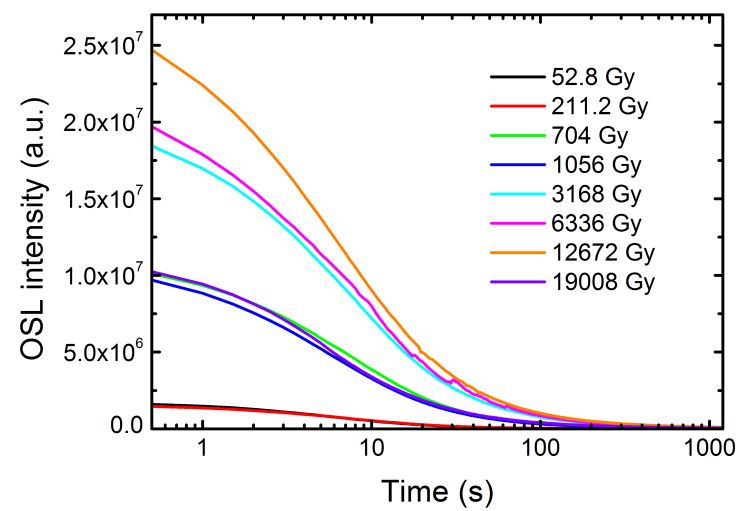

(a)

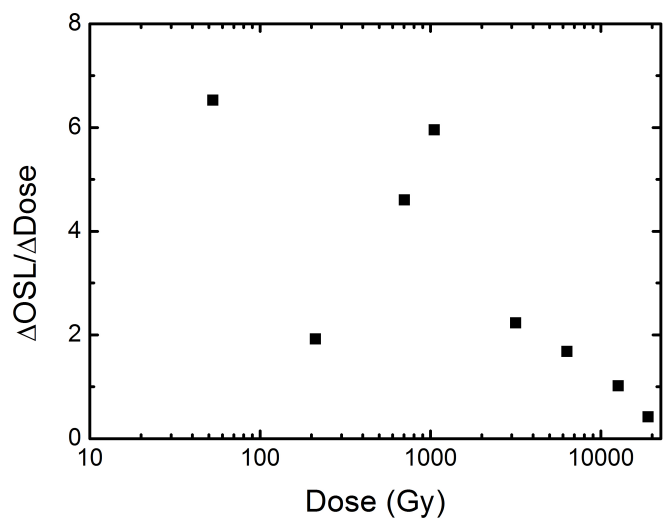

(c)

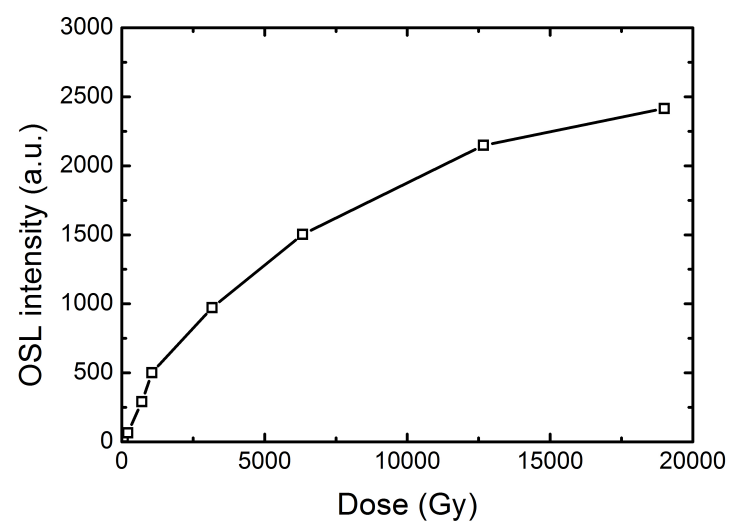

(b)

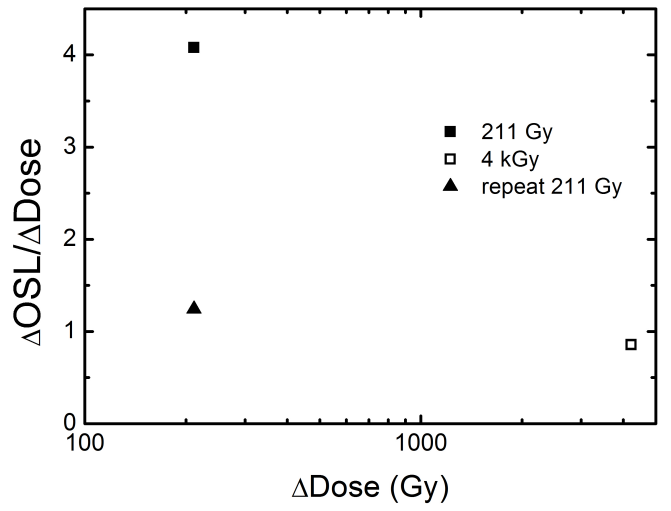

(d)

Figure 5.18: (a) Absolute OSL signal versus time for the different doses.

(b) The total OSL signal plotted versus the total dose.

(c) The change of the OSL signal ( $\triangle \mathrm{OSL})$ divided by the change of dose versus the total dose.

(d) $\Delta$ OSL $/ \Delta$ dose versus the change of the dose $(\Delta$ dose). 


\subsection{Conclusion}

PL, TL, RL and OSL were observed for polycrystalline $\mathrm{NaMgF}_{3}$ doped with Eu. Photoluminescence measurements show the presence of $\mathrm{Eu}^{2+}$ and $\mathrm{Eu}^{3+}$ ions in the $\mathrm{Eu}$ doped sample as well as an unknown defect centred around $470 \mathrm{~nm}$. PL measurements of the Eu doped samples before and after irradiation show that the total PL emission signal of the $\mathrm{Eu}^{2+}$ site decreases after heavy irradiation. The $\mathrm{Eu}^{3+}$ signal could not be detected due to the increased broad defect centred at $470 \mathrm{~nm}$ for the excitation spectra after irradiation. The $\mathrm{Eu}^{3+}$ transitions, when excited at $396 \mathrm{~nm}$, increase after irradiation with an initial dose of $19 \mathrm{kGy}$. This could be observed after subtracting the unknown defect centred at $470 \mathrm{~nm}$ in the PL spectra. The newly created $\mathrm{Eu}^{3+}$ ions are less distorted since the ratio of ${ }^{7} \mathrm{~F}_{2}:{ }^{7} \mathrm{~F}_{1}$ decreases from 2:1 before to $1: 1$ after irradiation. TL measurements for $\mathrm{NaMgF}_{3}$ doped with Eu show three peaks with a main peak at low temperatures $(\sim 363 \mathrm{~K})$. $\mathrm{Eu}^{2+}$ and $\mathrm{Eu}^{3+}$ were also observed in the RL spectra and their emission wavelengths are the same wavelengths as observed in the PL spectra. The integrated RL intensity for the $\mathrm{Eu}^{2+}$ ion increases until it reaches a maximum above 850 Gy. After this increase it decreases again until $13 \mathrm{kGy}$. The ratio of the broad peak centred at $400 \mathrm{~nm}$ to the sharp peak centred at $360 \mathrm{~nm}$ increases with a higher dose. This was also observed for the PL emission spectra and can be expalined with the creation of defects near the $\mathrm{Eu}^{2+}$ site. These defects shift the $5 \mathrm{~d}$ and $4 \mathrm{f}$ level which increases the transition of the $5 \mathrm{~d}$ level on top of the decrease of the $\mathrm{Eu}^{2+}$ signal. The RL signal of the $\mathrm{Eu}^{3+}$ is nearly independent of dose, which is a desirable property for a real-time dosimeter. For the unknown defect, a decrease of RL intensity versus dose was observed, which is due to the higher probability of filling traps with non-radiative recombination centres next to the unknown defect. The temperature dependent RL measurements for the $\mathrm{Eu}^{2+}$ ion are mainly dominated by detrapping deep traps. Hence, only corrections for this effect need to taken into account when using these materials as dosimeters. The value of the temperature dependence of the RL intensity is $0.11 \pm 0.08 \% / \mathrm{K}$ and the value is within the range as expected for dosimeters $(<0.4 \% / \mathrm{K})$. The shape of the $\mathrm{Eu}^{2+} \mathrm{RL}$ emission curve also changes with increasing temperature. The 
intensity of the sharp peak centred at $360 \mathrm{~nm}$ decreases with increasing temperature from room temperature up to $100{ }^{\circ} \mathrm{C}$ leading to a higher emission from the $5 \mathrm{~d}$ level. Due to an increase in temperature, the energy difference between the $4 \mathrm{f}$ to the $5 \mathrm{~d}$ level, $\Delta$, decreases and the probability of exciting into the $5 \mathrm{~d}$ level increases with higher temperature, making TL transition from the $4 \mathrm{f}$ to the $5 \mathrm{~d}$ level more likely. There was no shift in RL emission wavelengths with increasing temperatures for the $\mathrm{NaMgF}_{3}$ samples doped with Eu observed. The thermal coefficient was estimated using the average of the temperature dependent RL curve and was around $0.11 \pm 0.08 \% / \mathrm{K}$. The total OSL signal rapidly increases until $1 \mathrm{kGy}$ and decreases above $19 \mathrm{kGy}$. The change of the OSL signal divided by the change of the dose versus the total dose shows the same behaviour as the integrated RL signal versus the dose. This leads to the suggestion that this effect is due to the generation of the same traps.

For the $0.5 \%$ and $1 \% \mathrm{Mn}$ doped samples, an additional high distorted peak of a $\mathrm{Mn}^{2+}$ site was observed in the PL spectrum. The PL lifetime of the highly distorted $\mathrm{Mn}^{2+}$ peak centred at $500 \mathrm{~nm}$ was lower than the lifetime of the regular Mn site centred at $600 \mathrm{~nm}$. The transition of the distorted site more likely because it is only parity forbidden and therefore shows a lower lifetime. The TL glow curve for the Mn doped samples could be fitted with a deconvolution and consist of four peaks, both of the concentrations had two main peaks with similar peak temperatures and an activation energies of $0.5 \mathrm{eV}$. The high temperature peak above $608 \mathrm{~K}$ shifts towards higher temperatures for higher concentrations of Mn and with a higher initial dose. There was no PTTL observed. The RL spectra show that the ratio of the two $\mathrm{Mn}^{2+}$ sites decreases with increasing concentration. The decrease of the ratio of the distorted site to the regular site with increasing Mn concentration means there are less distorted defects in the sample with increasing Mn concentration. For the regular Mn site and both Mn concentrations, The integrated RL intensity increases until it reaches a maximum above a dose of $\sim 8.5 \mathrm{kGy}$. After this initial increase the RL signal decreases up to a dose of $13 \mathrm{kGy}$. For both Mn concentrations, the distorted site shows an increase of $\mathrm{I}_{R L}$ until it reaches a maximum above a dose of $\sim 5.3 \mathrm{kGy}$. After this dose the 
RL intensity decreases until a dose of $\sim 13 \mathrm{kGy}$. The temperature dependent RL intensity for the $\mathrm{Mn}$ doped samples depends more on the regular site because it has a higher RL signal as the distorted site. The RL intensity is temperature independent for the distorted site in the $0.5 \%$ sample. The $1 \%$ doped sample shows an increase of the RL signal for the regular site because of the increase of the probability of detrapping deep traps with increasing temperature. The thermal coefficient of the RL for the regular site is around $0.01 \pm 0.1 \% / \mathrm{K}$ for the $0.5 \% \mathrm{Mn}$ doped sample and $0.03 \pm 0.07 \% / \mathrm{K}$ for the $1 \%$ sample. This is a good value for application as a real-time dosimeter. 


\section{$6 \mathrm{NaMgF}_{3}$ nanoparticles}

The nanoparticles were either doped with $0.1 \%, 0.5 \%, 1 \%, 2 \%$ and $5 \%$ Eu or $1 \%$, $2 \%$ and $5 \% \mathrm{Mn}$. Their optical, radioluminescent and thermoluminescent properties will be discussed in this chapter. The samples were prepared with the method described in chapter 4.1 .2 [76]. The crystal structure is orthorhombic with the space group $P_{b n m}$ and from XRD measurements, the crystal size was calculated with the Scherrer formula from the XRD spectra shown in Fig. 4.1, which was $\sim 25 \mathrm{~nm}$. These nanoparticles had a smaller crystal size than the onces observed by Williams et al., which had an average size of 53 $\mathrm{nm}$ for the $\mathrm{Mn}$ samples, and $57 \mathrm{~nm}$ for the $1 \% \mathrm{Eu}$ and $77 \mathrm{~nm}$ for the $5 \% \mathrm{Eu}$ doped samples [10]. It is not clear why the size of the nanoparticles is smaller. This might be caused by the production process. To perform PL, PL lifetime and RL measurements, the nanoparticles were pressed into pellets with a diameter of $\sim 6 \mathrm{~mm}$ and a thickness between $0.5 \mathrm{~mm}$ and $1.5 \mathrm{~mm}$. The appearance of the pressed pellets was whitish. The influence of the concentration of the dopant on the PL spectra, PL lifetime, TL and RL measurements will be examined. There was no OSL observed for any of the nanoparticles.

\subsection{Photoluminescence and PL lifetime measurements}

PL measurements were performed with the Jobin-Yvon FluoroLog ${ }^{\circledR}$. 


\subsubsection{Eu doped $\mathrm{NaMgF}_{3}$ nanoparticles}

The PL spectrum of $\mathrm{NaMgF}_{3}: 5 \% \mathrm{Eu}$ is plotted in Fig. 6.1 $(\mathrm{a}+\mathrm{b})$. The PL excitation spectra detected at $590 \mathrm{~nm}$ arises mainly from the ${ }^{7} \mathrm{~F}_{1}$ transition of $\mathrm{Eu}^{3+}$ (solid curve in Fig. 6.1 (a)). $\mathrm{Eu}^{2+}$ was detected for an emission at $360 \mathrm{~nm}$ (dashed curve in Fig. 6.1(a)). When exciting the sample at $396 \mathrm{~nm} \mathrm{Eu}^{3+}$ emission was observed (Fig. 6.1(b)) and when exciting at $256 \mathrm{~nm} \mathrm{Eu}^{2+}$ emission was detected (see inset in Fig. 6.1(b)). The sharp peak centred at $360 \mathrm{~nm}$ in the $\mathrm{PL}$ emission spectrum is due to the transition of ${ }^{6} \mathrm{P}_{7 / 2}$ and ${ }^{6} \mathrm{P}_{5 / 2}$ excited states to the ${ }^{8} \mathrm{~S}_{7 / 2}$ ground state and the broad peak centred at $380 \mathrm{~nm}$ is from the band emission from the $4 \mathrm{f}^{6} 5 \mathrm{~d}\left(\mathrm{E}_{g}\right)$ excited states to the $4 \mathrm{f}^{7}$ ground state from the $\mathrm{Eu}^{2+}$ ion. When exciting at $396 \mathrm{~nm}$ the PL emission mainly arises from the ${ }^{5} \mathrm{D}_{1}$ and ${ }^{5} \mathrm{D}_{0}$ level. The solid curve in the PL emission is from the $5 \% \mathrm{Eu}$ doped sample and the dashed curve from the $1 \%$ doped sample (Fig. 6.1(b)). The ratio of $\mathrm{Eu}^{2+} / \mathrm{Eu}^{3+}$ is $1.8: 1$ for the $5 \%$ doped sample. The transitions of the excited states to the ground state are indicated in Fig. 6.1 $(\mathrm{a}+\mathrm{b})$ for the $\mathrm{Eu}^{2+}$ and $\mathrm{Eu}^{3+}$ emission.

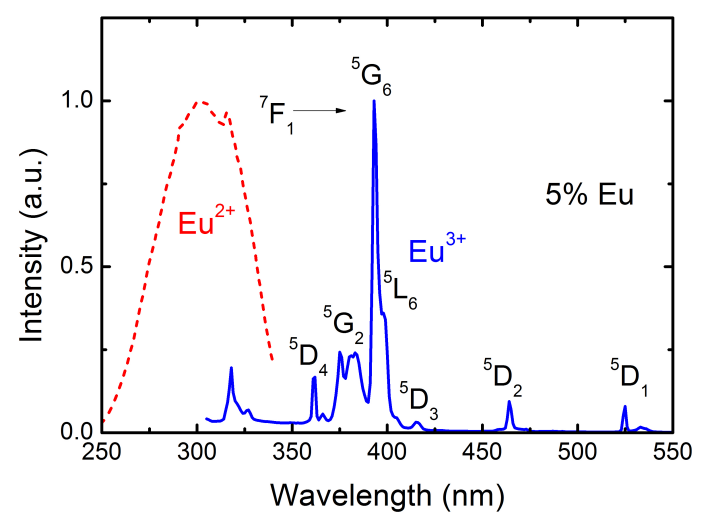

(a)

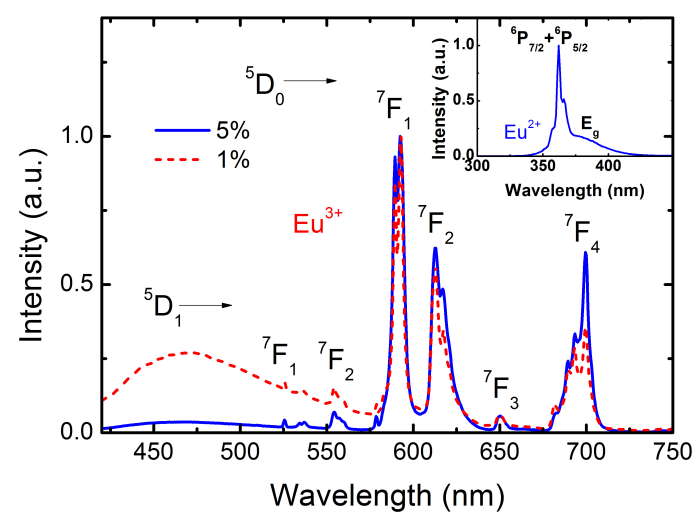

(b)

Figure 6.1: (a) PL excitation for the $\mathrm{Eu}^{2+}\left(\lambda_{e m}=360 \mathrm{~nm}\right.$ - dashed curve $)$ and $\mathrm{Eu}^{3+}\left(\lambda_{e m}=\right.$ $592 \mathrm{~nm}$ - solid curve) and (b) emission $\left(\lambda_{e x}=396 \mathrm{~nm}\right.$; inset $\left.\lambda_{e x}=256 \mathrm{~nm}\right)$ spectra for $\mathrm{NaMgF}_{3}$ doped with $1 \% \mathrm{Eu}$ (dashed curve) and $5 \% \mathrm{Eu}$ (solid curve). 
The PL spectra for low temperatures are plotted in Fig. 6.2. An additional broad peak between the $\mathrm{Eu}^{2+}$ and $\mathrm{Eu}^{3+}$ emission appears. This unknown defect has a maximum at $\sim 470 \mathrm{~nm}$ and the emission of this defect is weaker at room temperature. The excitation spectrum of the defect at $\sim 470 \mathrm{~nm}$ is shown in the inset and it consists of one peak at $360 \mathrm{~nm}$ for the measurements at $300 \mathrm{~K}$. At $10 \mathrm{~K}$ the excitation spectrum consists out of two peaks, one centred at $300 \mathrm{~nm}$ and one at $360 \mathrm{~nm}$. This new band at $470 \mathrm{~nm}$ can be assigned to some defects. The precise origin of these defects is unknown, but can probably be ascribed to the same defect previously observed in $\mathrm{NaMgF}_{3}: \mathrm{Eu}$ bulk material (see Fig. $5.3(\mathrm{~b}))$. The emission of the $\mathrm{Eu}^{3+}$ are also weaker at room temperature. This leads to the assumption that non-radiative recombinations increase with increasing temperature. The ratio of $I_{{ }^{7} F_{2}} / I_{{ }^{7} F_{1}}$ is $1: 1.6$ for the $0.1 \%$ sample, $1: 1$ for the $0.5 \%$ sample, $1: 1.3$ for the $1 \%$ one and further decreasing to 1:1.2 for the $5 \%$ sample. The average crystal environment of $\mathrm{Eu}^{3+}$ seems to get more distorted with increasing $\mathrm{Eu}^{3+}$ concentration. This was also later observed in this chapter when calculating the average PL lifetime and it will be shown that with increasing $\mathrm{Eu}^{3+}$ concentration, the energy transfer from $\mathrm{Eu}^{3+}$ away from the surface to $\mathrm{Eu}^{3+}$ sites near the surface increases [10, 11, 12, 22, 83]. 


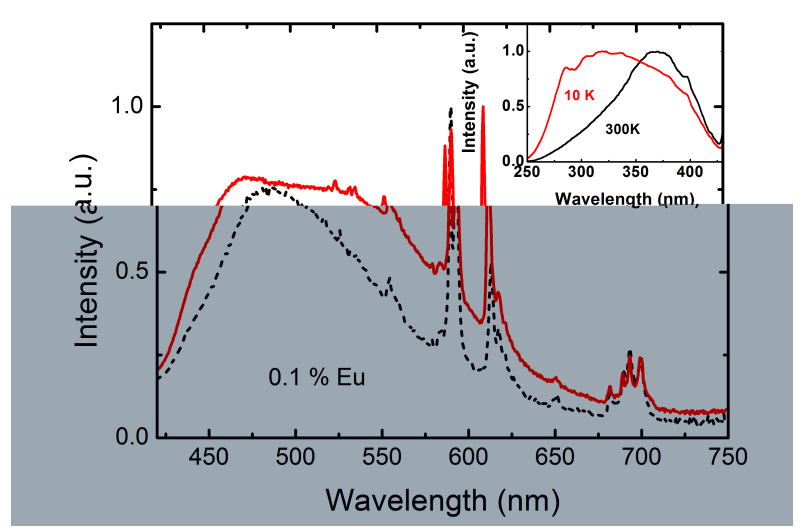

(a)

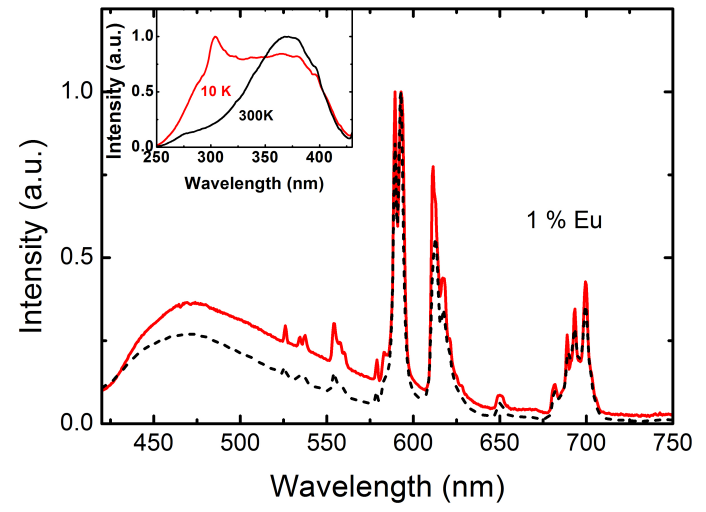

(c)

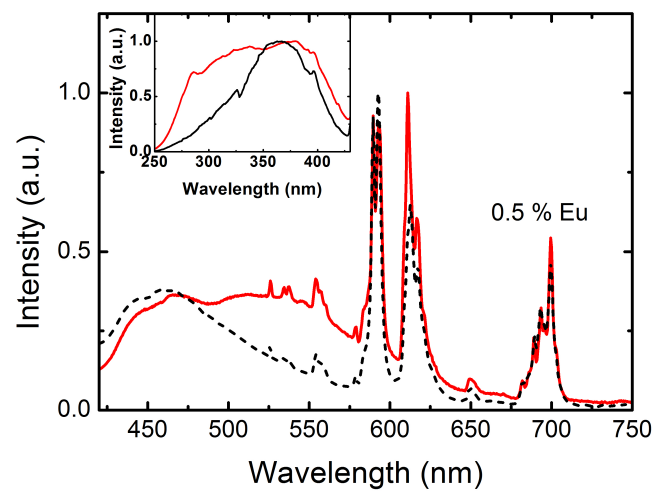

(b)

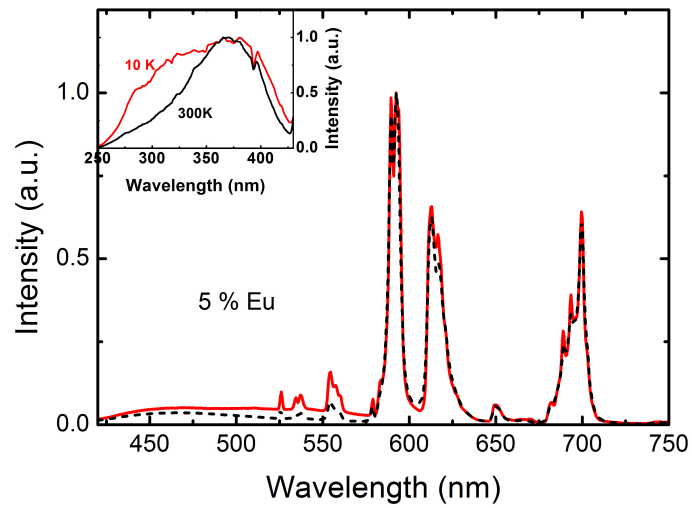

(d)

Figure 6.2: (a) PL emission spectra for $0.1 \%$, (b) $0.5 \%$. (c) $1 \%$ and (d) $5 \%$ Eu doped $\mathrm{NaMgF}_{3}$ at $\lambda_{e x}=396 \mathrm{~nm}$ at $10 \mathrm{~K}$ (solid curve) and at room temperature (dashed curve). Inset: PL excitation spectra detected at $\lambda_{e m}=450 \mathrm{~nm}$ for the different Eu concentrations of $\mathrm{NaMgF}_{3}$.

Temperature dependent PL lifetime measurements from the $\mathrm{Eu}^{3+}$ ion were performed for the $1 \% \mathrm{Eu}$ (Fig. 6.3) and $5 \% \mathrm{Eu}$ (Fig. 6.4) doped sample. The measurements were performed while cooling the samples down until $10 \mathrm{~K}$ with a cryostat and then increasing the temperature in $30 \mathrm{~K}$ steps. The PL decay can be fitted with multiple exponential components and the average lifetime was estimated as mentioned in section 3.2.4. 
The radiative lifetime from $\mathrm{Eu}^{3+}$ can be estimated from the ${ }^{5} \mathrm{D}_{0} \rightarrow{ }^{7} \mathrm{~F}_{J}$ emission as mentioned in section 3.2.4. $\tau_{\text {rad }}$ was calculated employing the corrected PL emission spectra of the $\mathrm{Eu}^{3+}$ transition and subtracting the signal from the broad unknown defect centred at $\sim 470 \mathrm{~nm}$. These average lifetimes are listed in Tab. 6.1. $\tau_{1}$ and $\tau_{2}$ seemed to be less temperature dependent than $\tau_{3}$ (Fig. 6.3(a)). The values of $\tau_{3}$ are always bigger than the radiative lifetime of $9.9 \pm 1.0 \mathrm{~ms}$ (values up to $33.5 \pm 3.4 \mathrm{~ms}$ for $10 \mathrm{~K}$ ) and strongly temperature dependent. For low temperatures down to $10 \mathrm{~K}$, the average lifetime was as high as $13.8 \pm 1.4 \mathrm{~ms}$ when taking all three components into account. This is much higher than the measured radiative lifetime of around $9.9 \pm 1.0 \mathrm{~ms}$. Therefore, it is likely that $\tau_{3}$ is from the defect which causes the broad emission peak centred at $470 \mathrm{~nm}$. Hence, these values were not added in the summation of the $\mathrm{Eu}^{3+}$ lifetime (Fig. 6.3(b)). This gives an average PL lifetime of around $7.85 \pm 0.79 \mathrm{~ms}$ at room temperature. This effect was already reported for $\mathrm{NaMgF}_{3}$ nanoparticles [10, 11, 12, 22, 83. Williams et al. reported that the temperature dependence of $\tau_{3}$ might occur because thermal quenching resulting in two decays: One radiative and one thermally active non-radiative. They suggested that $\tau_{3}$ can be estimated from the following equation,

$$
\frac{1}{\tau_{3}}=\frac{1}{\tau_{3,0}}+f \times e^{-\frac{E_{A}}{k_{B} T}}
$$

with $\tau_{3,0}$ as the intrinsic effective decay time at $0 \mathrm{~K}, \mathrm{E}_{A}$ the activation energy, $\mathrm{k}_{B}$ the Boltzmann's constant and $f$ the attempt frequency [10]. At $592 \mathrm{~nm} \tau_{3,0}$ is $32.5 \pm 3.3 \mathrm{~ms}$ and $\mathrm{E}_{A}$ is $104 \pm 36 \mathrm{meV}$. 


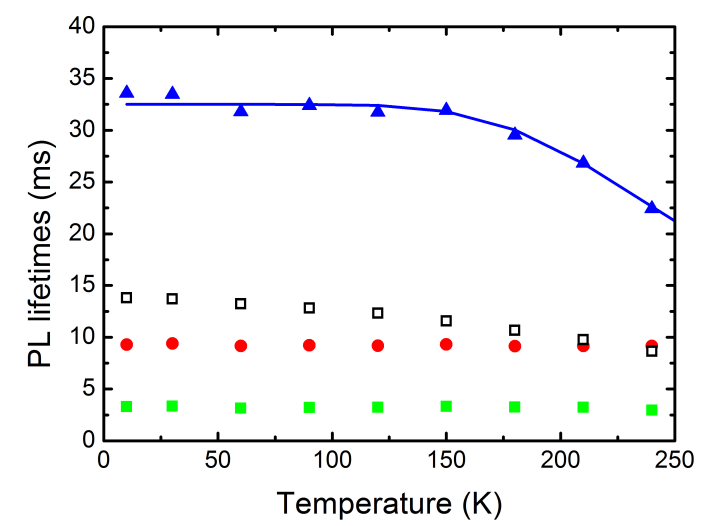

(a)

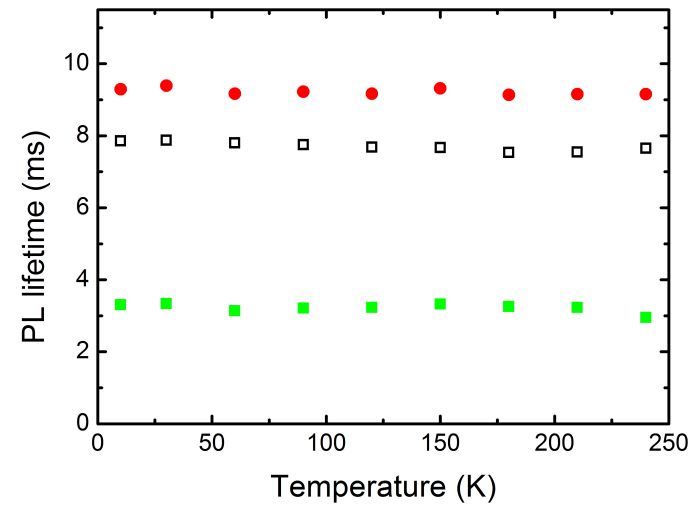

(b)

Figure 6.3: (a) Temperature dependent average PL lifetime of $1 \% \mathrm{Eu}$ doped $\mathrm{NaMgF}_{3}$ $\left(\lambda_{e m}=592 \mathrm{~nm} ; \lambda_{e x}=375 \mathrm{~nm}\right)$ from the three exponential fit of the data with only the $\tau_{1}$ (filled squares), $\tau_{2}$ (filled circles), $\tau_{3}$ (filled triangles) and the average lifetime, $\tau_{123, a v e}$ (open squares). $\tau_{3}$ was fitted using equation 6.1 .

(b) Temperature dependent average PL lifetime of $1 \%$ Eu doped $\mathrm{NaMgF}_{3}$ from the three exponential fit of the data with only the $\tau_{1}$ (filled squares), $\tau_{2}$ (filled circles) and the average lifetime, $\tau_{12, a v e}$ (open squares) when only using the first and the second component.

Fig. 6.4(a) shows the average PL lifetime of the three components when fitting the decay curve multi - exponential and Fig. 6.4(b) shows the fit with only two of the components for the $5 \% \mathrm{Eu}$ doped sample. When adding all three components to the lifetime, $\tau_{123, \text { ave }}$, gets as high as $13.9 \pm 1.4 \mathrm{~ms}$ at $10 \mathrm{~K}$ with a $\tau_{3}$ component of $31.3 \pm 3.1 \mathrm{~ms}$. Therefore, the $\tau_{3}$ component was excluded from $\tau_{\text {ave }}$. Fig. 6.4(b) shows that $\tau_{12, \text { ave }}$ gets as low as $7.4 \pm 0.7 \mathrm{~ms}$ at room temperature. $\tau_{3}$ was also fitted with equation 8.1 and at $592 \mathrm{~nm} \tau_{3,0}$ is $17.9 \pm 1.8 \mathrm{~ms}$ with a calculated $\mathrm{E}_{A}$ of $95 \pm 40 \mathrm{meV}$ (Fig. 6.4(a)). This value of $\mathrm{E}_{A}$ is the same as within the experimental uncertainty for the $1 \%$ doped sample where an $\mathrm{E}_{A}$ of $104 \pm 36 \mathrm{meV}$ was calculated. Williams et al. reported an $\mathrm{E}_{A}$ of $169 \pm 39 \mathrm{meV}$ for the $1 \%$ Eu doped $\mathrm{NaMgF}_{3}$ sample and an $\mathrm{E}_{A}$ of $206 \pm 49 \mathrm{meV}$ for the $5 \%$ sample measured at 592 
$\mathrm{nm}$. For the measurement at $500 \mathrm{~nm}$ Williams et al. reported an value of $139 \pm 20 \mathrm{meV}$ for $\mathrm{E}_{A}$. This $\mathrm{E}_{A}$ is closer to the values calculated for the nanoparticles in this thesis which have a smaller crystal size [10].

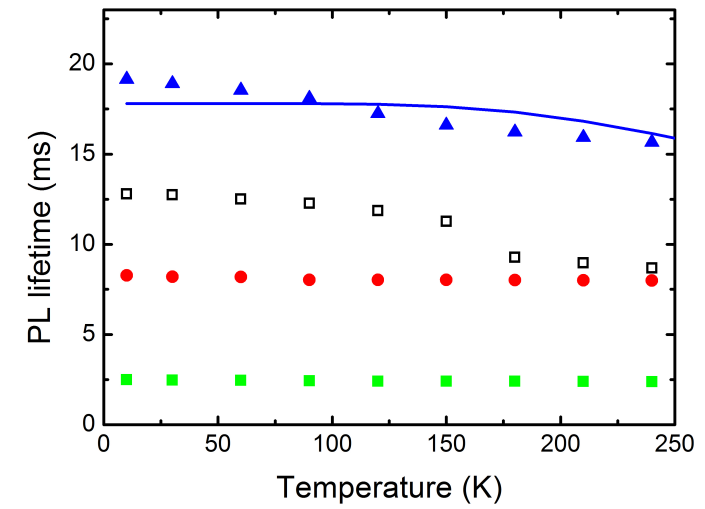

(a)

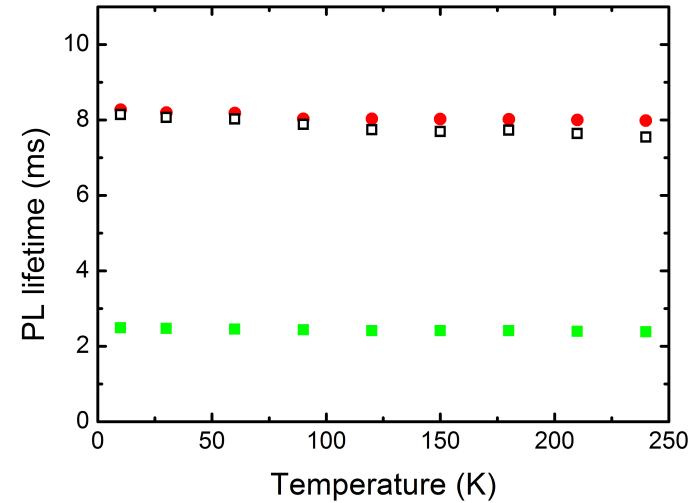

(b)

Figure 6.4: (a) Temperature dependent average PL lifetime of $5 \% \mathrm{Eu}$ doped $\mathrm{NaMgF}_{3}$ $\left(\lambda_{e m}=592 \mathrm{~nm} ; \lambda_{e x}=375 \mathrm{~nm}\right)$ from the three exponential fit of the data with only the $\tau_{1}$ (filled squares), $\tau_{2}$ (filled circles), $\tau_{3}$ (filled triangles) and the average lifetime, $\tau_{123, \text { ave }}$ (open squares). $\tau_{3}$ was fitted using equation 6.1 .

(b) Temperature dependent average PL lifetime of $5 \%$ Eu doped $\mathrm{NaMgF}_{3}$ from the three exponential fit of the data with only the $\tau_{1}$ (filled squares), $\tau_{2}$ (filled circles) and the average lifetime, $\tau_{12, a v e}$ (open squares) when only using the first and the second component.

Table 6.1: Summary of the different measured PL lifetimes, $\tau_{12, \text { ave }}$, the radiative lifetime, $\tau_{\text {rad }}$, and the quantum efficiency, $\eta$, for $\mathrm{NaMgF}_{3}$ doped with different concentrations of Eu.

\begin{tabular}{|l|l|l|l|}
\hline Concentration $(\%)$ & $\tau_{12, \text { ave }}(\mathrm{ms})$ & $\tau_{\text {rad }}(\mathrm{ms})$ & $\eta(\%)$ \\
\hline \hline & $0.64 \pm 0.06(\mathrm{em} @ 360 \mathrm{~nm})$ & & \\
0.1 & $0.51 \pm 0.05(\mathrm{em} @ 380 \mathrm{~nm})$ & & \\
\hline
\end{tabular}

Continued on next page 
Table 6.1 - Continued from previous page

\begin{tabular}{|c|c|c|c|}
\hline Concentration (\%) & $\tau_{12, \text { ave }}(\mathrm{ms})$ & $\tau_{\text {rad }}(\mathrm{ms})$ & $\eta(\%)$ \\
\hline & $\begin{array}{l}49.4 \pm 4.9(\mathrm{em} @ 500 \mathrm{~nm}) \\
9.5 \pm 1.0(\mathrm{em} @ 592 \mathrm{~nm})\end{array}$ & 11.0 & 86.4 \\
\hline 0.5 & $\begin{array}{l}0.60 \pm 0.06(\mathrm{em} @ 360 \mathrm{~nm}) \\
0.46 \pm 0.05(\mathrm{em} @ 380 \mathrm{~nm}) \\
44.5 \pm 4.5(\mathrm{em} @ 500 \mathrm{~nm}) \\
9.1 \pm 0.9(\mathrm{em} @ 592 \mathrm{~nm})\end{array}$ & 9.1 & 99.7 \\
\hline 1 & $\begin{array}{l}0.62 \pm 0.06(\mathrm{em} @ 360 \mathrm{~nm}) \\
0.50 \pm 0.05(\mathrm{em} @ 380 \mathrm{~nm}) \\
41.7 \pm 4.2(\mathrm{em} @ 500 \mathrm{~nm}) \\
7.9 \pm 0.8(\mathrm{em} @ 592 \mathrm{~nm})\end{array}$ & 9.9 & 79.5 \\
\hline 2 & $\begin{array}{l}0.67 \pm 0.07(\mathrm{em} @ 360 \mathrm{~nm}) \\
0.52 \pm 0.05(\mathrm{em} @ 380 \mathrm{~nm}) \\
39.2 \pm 3.9(\mathrm{em} @ 500 \mathrm{~nm}) \\
7.36 \pm 0.74(\mathrm{em} @ 592 \mathrm{~nm})\end{array}$ & 10.4 & 71.0 \\
\hline 5 & $\begin{array}{l}0.65 \pm 0.06(\mathrm{em} @ 360 \mathrm{~nm}) \\
0.50 \pm 0.05(\mathrm{em} @ 380 \mathrm{~nm}) \\
35.5 \pm 3.6(\mathrm{em} @ 500 \mathrm{~nm}) \\
7.34 \pm 0.73(\mathrm{em} @ 592 \mathrm{~nm})\end{array}$ & 9.2 & 79.4 \\
\hline
\end{tabular}

PL lifetime measurements for the $\mathrm{Eu}^{3+}$ were performed with the Jobin-Yvon FluoroLog ${ }^{\circledR}$ and for the $\mathrm{Eu}^{2+}$ with the Cary50. A summary of the different lifetimes for the different Eu-ions in $\mathrm{NaMgF}_{3}$ is listed in Tab. 6.1 and Fig. 6.5 shows the lifetimes for an emission at 592 (a) and $500 \mathrm{~nm}$ (b). The measurements for the $\mathrm{Eu}^{2+}$ were performed for an emission at $360 \mathrm{~nm}$ and $380 \mathrm{~nm}\left(\lambda_{e x}=256 \mathrm{~nm}\right)$. For an emission wavelength of $592 \mathrm{~nm}$ the lifetime for $\mathrm{Eu}^{3+}$ ion were measured $\left(\lambda_{e x}=375 \mathrm{~nm}\right)$. The average lifetimes for the unknown defect centred at $500 \mathrm{~nm}$ were measured at an excitation wavelength of $375 \mathrm{~nm}$. 


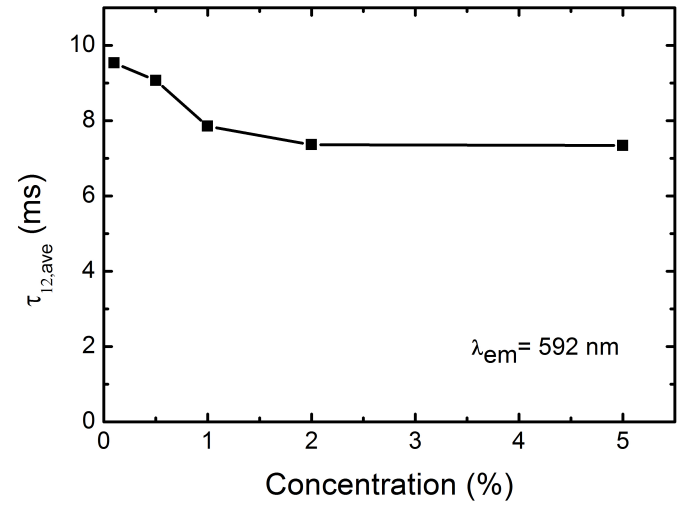

(a)

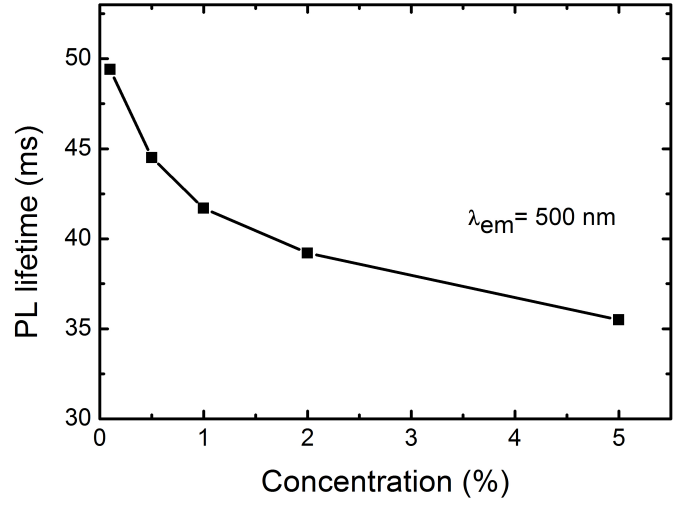

(b)

Figure 6.5: (a) Average PL lifetime for $\mathrm{Eu}^{3+}\left(\lambda_{e m}=592 \mathrm{~nm}, \lambda_{e x}=375 \mathrm{~nm}\right)$ doped $\mathrm{NaMgF}_{3}$ nanoparticles against the rare earth concentration.

(b) The PL lifetime for the unknown broad peak was measured at $\lambda_{e m}=500$ $\mathrm{nm}$ and $\lambda_{e x}=375 \mathrm{~nm}$.

All measurements were done at room temperature.

A radiative lifetime for the $\mathrm{Eu}^{3+}$ ion of up to $11.2 \pm 1.1 \mathrm{~ms}$ was calculated for the $0.1 \% \mathrm{Eu}$ doped sample and the lifetime was as low as $9.1 \pm 0.9 \mathrm{~ms}$ for the $0.5 \%$ doped sample (see Tab. 6.1). Fig. 6.6(a) shows a PL lifetime decay curve for the $5 \% \mathrm{Eu}$ doped $\mathrm{NaMgF}_{3}$ sample for an emission wavelength of $590 \mathrm{~nm}$ (solid curve) and the dashed curve represents the exponential fit $\left(\lambda_{e x}=375 \mathrm{~nm}\right)$. A decrease of the decay curves for the different $\mathrm{Eu}^{3+}$ concentrations is illustrated in Fig. 6.6(b). 


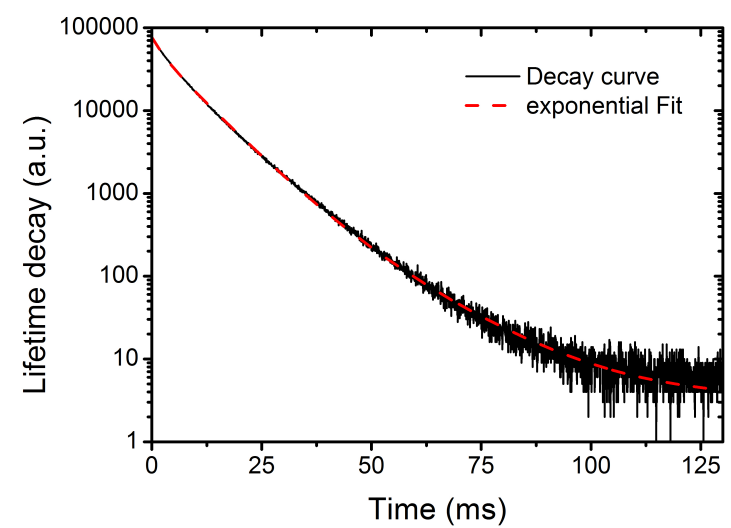

(a)

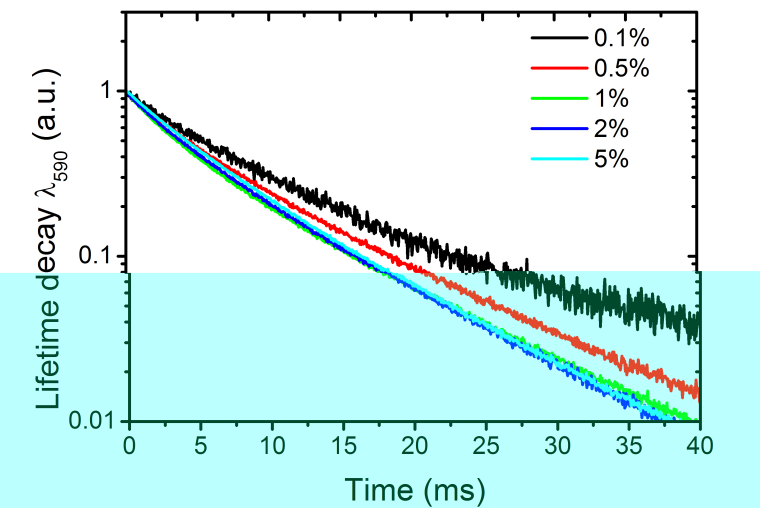

(b)

Figure 6.6: (a) PL lifetime decay of $5 \%$ Eu doped $\mathrm{NaMgF}_{3}$ at $\lambda_{e m}=590 \mathrm{~nm}$ (solid curve). The dashed curve is the exponential fit. The lifetime was measured at $\lambda_{e x}=$ $375 \mathrm{~nm}$.

(b) The lifetime decay curves at $\lambda_{e m}=590 \mathrm{~nm}$ emission for the different $\mathrm{Eu}$ concentrations in $\mathrm{NaMgF}_{3}$.

$\tau_{\text {ave }}$ does not change significantly with the Eu concentration for $\mathrm{Eu}^{2+}$ ions but it decreases with increasing $\mathrm{Eu}^{3+}$ concentration. The resultant quantum efficiencies, $\eta$, are as high as 99.7 \%. A systematic decrease in the Eu PL lifetimes, $\tau_{12, \text { ave }}$, can be attributed to energy transfer between $\mathrm{Eu}^{3+}$ sites and a high concentration of surface non-radiative recombination sites that results in a lower PL lifetime for $\mathrm{Eu}^{3+}$ at the surface. The decrease in $\tau_{500}$ for high Eu concentrations observed in this study suggests that there is also energy transfer between the unknown defects to sites with higher non-radiative decay rates. The average PL lifetime of the unknown defect in the polycrystalline bulk materials was around $67 \mathrm{~ms}$ for an emission wavelength of $460 \mathrm{~nm}$. This lifetime is larger than the calculated lifetimes of $\mathrm{Eu}^{2+}$ and $\mathrm{Eu}^{3+}$. These non-radiative decay surface sites were previously observed in other nanoparticles and it was shown that only luminescent ions near the surface will experience non-radiative decay [85, 86]. This reduction in the PL lifetime was observed for $\mathrm{LaF}_{3}$ nanoparticles and also reported for $\mathrm{NaMgF}_{3}$ ones [10, 11, 12, 22, 83]. The energy transfer 
occurs in terms of a transfer from $\mathrm{Eu}^{3+}$ in Eu sites near the surface or $\mathrm{Eu}^{3+}$ ion sites away from the surface. This energy transfer between $\mathrm{Eu}^{3+}$ ions could occur by a dipole-dipole or dipole-quadrupole mechanism which have $\mathrm{a}^{-6}$ or $^{-8}$ dependency, respectively [91]. This means for the dipole-dipole mechanism as the average distance between nuclei decreases cubically with the $\mathrm{Eu}^{3+}$ concentration the energy transfer increases quadratically with $\mathrm{Eu}^{3+}$ concentration. It seems for $\tau_{12 \text {,ave }}$ that from a certain concentration the amount of extra $\mathrm{Eu}$ does not matter anymore because the energy transfer is already very efficient and hence does not cause an extra decrease in lifetime, see Fig. 6.5(a). For the polycrystalline $\mathrm{NaMgF}_{3}: \mathrm{Eu}$ bulk materials, an average PL lifetime of $0.63 \pm 0.06 \mathrm{~ms}$ for an emission wavelength of $360 \mathrm{~nm}, 0.42 \pm 0.04 \mathrm{~ms}$ for an emission wavelength of $380 \mathrm{~nm}$ and $11.1 \pm 0.1 \mathrm{~ms}$ for an emission wavelength of $592 \mathrm{~nm}$ was calculated. The PL lifetimes for the $\mathrm{Eu}^{2+}$ emission are similar in the nanoparticles. The lifetime of the $\mathrm{Eu}^{3+}$ lifetime is slightly higher in the bulk materials.

The Judd-Ofelt parameters, $\Omega_{2}$ and $\Omega_{4}$, for the $\mathrm{Eu}^{3+}$ ions were also calculated [65, 66, 92]. The parameters were estimated from equation 3.4, where $\mathrm{I}_{0-J}$ are the intensity values from the PL spectra in Fig. 6.2 at room temperature after subtracting the unknown broad defect centred at $470 \mathrm{~nm}$. The resulting $\Omega_{2}$ and $\Omega_{4}$ values are listed in Tab. 6.2. $\Omega_{2}$ and $\Omega_{4}$ are $6.87 \times 10^{-20} \mathrm{~cm}^{2}$ and $25.0 \times 10^{-20} \mathrm{~cm}^{2}$ for the $1 \%$ doped Eu sample and $7.52 \times 10^{-20}$ $\mathrm{cm}^{2}$ and $27.9 \times 10^{-20} \mathrm{~cm}^{2}$ for $5 \%$ Eu. Williams et al. calculated the values of $\Omega_{2}$ and $\Omega_{4}$ as $6.95 \times 10^{-20} \mathrm{~cm}^{2}$ and $15.7 \times 10^{-20} \mathrm{~cm}^{2}$ for $1 \% \mathrm{Eu}$ and $11.3 \times 10^{-20} \mathrm{~cm}^{2}$ and $22.8 \times 10^{-20}$ $\mathrm{cm}^{2}$ for $5 \% \mathrm{Eu}$ doped $\mathrm{NaMgF}_{3}$ [10]. These values are also summarised in Tab. 6.2. It was observed that for smaller nanoparticles the resultant $\Omega_{2}$ is also smaller. This indicates that, on average, the $\mathrm{Eu}^{3+}$ is in less distorted sites [10]. It was not possible to see any dependence of $\Omega_{2}$ of the Eu concentration for the different nanoparticles. It was reported that $\Omega_{2}$ is to be found in ranges of $\sim 1 \times 10^{-20} \mathrm{~cm}^{2}$ to $33 \times 10^{-20} \mathrm{~cm}^{2}$ in other nanoparticle compounds [22, 93, 94, 95]. Brito et al. reported that $\Omega_{2}$ is connected to short range effects and if the symmetry of the $\mathrm{Eu}^{3+}$ site is reduced $\Omega_{2}$ will increase 93 . Therefore, the higher $\Omega_{2}$ is, the more $\mathrm{Eu}^{3+}$ is in a distorted site. $\mathrm{Eu}^{3+}$ is more distorted near the surface which 
was already observed for other nanoparticle compounds [10, 11, 12, 22]. $\Omega_{4}$ is connected with long range effects [93], and it is found in ranges from $(0.2$ to 13$) \times 10^{-20} \mathrm{~cm}^{2}$. It is not clear why $\Omega_{4}$ is higher than ranges found in other compounds.

Table 6.2: The Judd-Ofelt parameters $\Omega_{2}$ and $\Omega_{4}$ for ${ }^{5} \mathrm{D}_{0} \rightarrow{ }^{7} \mathrm{~F}_{2}$ transitions for different concentrations of Eu doped $\mathrm{NaMgF}_{3}$ nanoparticles measured using the PL emission.

\begin{tabular}{|l|l|l|l|}
\hline Concentration $(\%)$ & $\Omega_{2}\left(10^{-20} \mathrm{~cm}^{2}\right)$ & $\Omega_{4}\left(10^{-20} \mathrm{~cm}^{2}\right)$ & $\Omega_{2}\left(10^{-20} \mathrm{~cm}^{2}\right)[10]$ \\
\hline \hline 0.1 & $5.3 \pm 0.5$ & $22.2 \pm 2.2$ & \\
\hline 0.5 & $8.3 \pm 0.8$ & $26.7 \pm 2.7$ & \\
\hline 1 & $6.9 \pm 0.7$ & $25.0 \pm 2.5$ & 6.95 \\
\hline 2 & $5.9 \pm 0.6$ & $24.9 \pm 2.5$ & \\
\hline 5 & $7.5 \pm 0.8$ & $27.9 \pm 2.8$ & 11.3 \\
\hline
\end{tabular}

\subsubsection{Mn doped $\mathrm{NaMgF}_{3}$ nanoparticles}

The PL emission spectrum for $\mathrm{NaMgF}_{3}$ doped with $5 \% \mathrm{Mn}$ is plotted in Fig. 6.7(a) and the excitation spectrum for different concentrations of $\mathrm{Mn}$ is shown in Fig. 6.7(b). $\mathrm{NaMgF}_{3}$ nanoparticles show a distorted peak centred at $500 \mathrm{~nm}$ in the PL spectrum. This was also observed for the $\mathrm{NaMgF}_{3}$ bulk materials. For the excitation spectrum was detected for an emission wavelength of $500 \mathrm{~nm}$ for the distorted site and $600 \mathrm{~nm}$ for the regular site (Fig. 6.7(a)). The emission spectra was excited at $396 \mathrm{~nm}$ (Fig. 6.7(b)). For the $\mathrm{NaMgF}_{3}: \mathrm{Mn}$ bulk materials, the PL spectra was measured for the different Mn concentrations and it was observed that the distorted site centred at $500 \mathrm{~nm}$ increases for smaller concentrations of the dopant in the material (see Fig. 5.6(b)). For the nanoparticles, it was observed that the PL intensity of the Mn site centred at $500 \mathrm{~nm}$ increases for Mn concentrations and reaches a maximum after a concentration of $2 \% \mathrm{Mn}$. After this initial increase the PL intensity starts to decrease until a concentration of $5 \% \mathrm{Mn}$. 


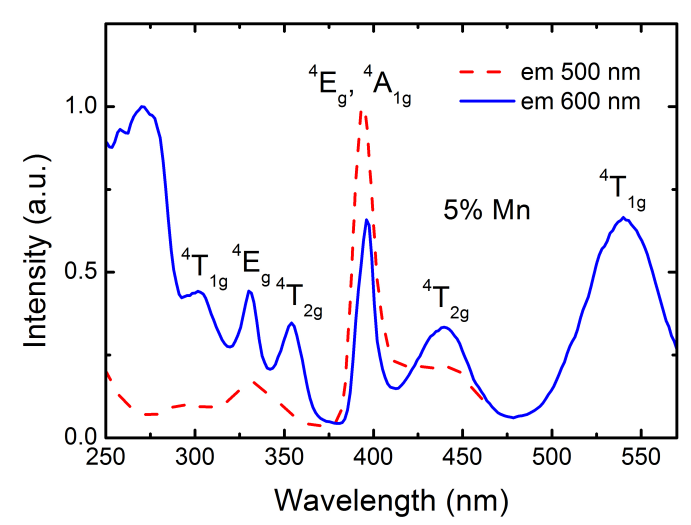

(a)

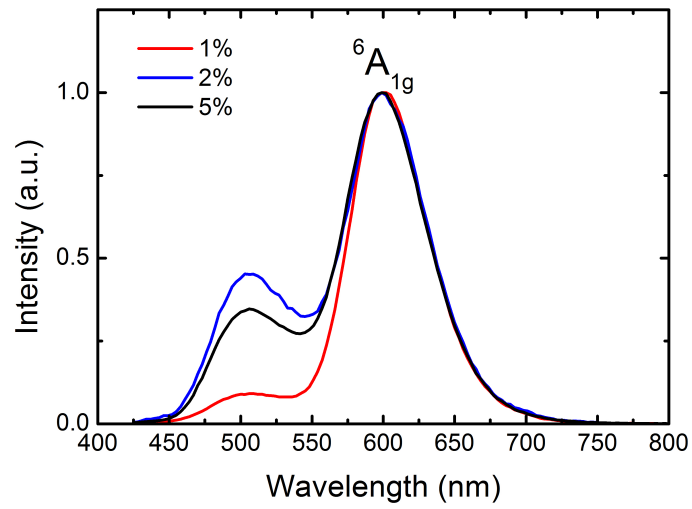

(b)

Figure 6.7: (a) PL excitation spectra for $5 \% \mathrm{Mn}$ doped $\mathrm{NaMgF}_{3}$ at $\lambda_{e m}=500 \mathrm{~nm}$ (dashed curve) and $\lambda_{e m}=600 \mathrm{~nm}$ (solid curve) for the distorted $\mathrm{Mn}^{2+}$ site.

(b) PL emission spectrum for $\mathrm{NaMgF}_{3}$ doped with $1 \% \mathrm{Mn}$ (red), $2 \%$ (blue) and $5 \%$ Mn (black) excited at $396 \mathrm{~nm}$.

Average PL lifetime was measured when exciting the sample at $465 \mathrm{~nm}$ for the regular $\mathrm{Mn}^{2+}$ site $\left(\lambda_{e m}=600 \mathrm{~nm}\right)$ and the distorted $\mathrm{Mn}^{2+}$ site $\left(\lambda_{e m}=500 \mathrm{~nm}\right)$. These measurements were carrier out with the Jobin-Yvon FluoroLog ${ }^{\circledR}$. The average PL lifetime, $\tau_{\text {ave }}$, could be fitted in a two - exponential decay (the same as the bulk materials). PL lifetime measurements showed a dependence of $\tau_{\text {ave }}$ on the Mn concentrations (Fig. 6.9 and Tab. 6.3). The highest lifetime was measured for the $2 \%$ doped sample and $\tau_{\text {ave }}$ was the lowest for the $1 \%$ doped sample (Fig. 6.8 $(\mathrm{a}+\mathrm{b})$ ). The average PL lifetime for the distorted $\mathrm{Mn}^{2+}$ site ranged from $11.1 \pm 1.1 \mathrm{~ms}$ until $11.6 \pm 1.2 \mathrm{~ms}$. It was lower than the calculated lifetime of the regular site which ranges from $62 \pm 6 \mathrm{~ms}$ until $80.1 \pm 8.0 \mathrm{~ms}$ (Tab. 6.3). The lower $\tau_{\text {ave }}$ for the distorted site may suggest that these sites are near a non-radiative recombination site, which makes the transition more allowed. 


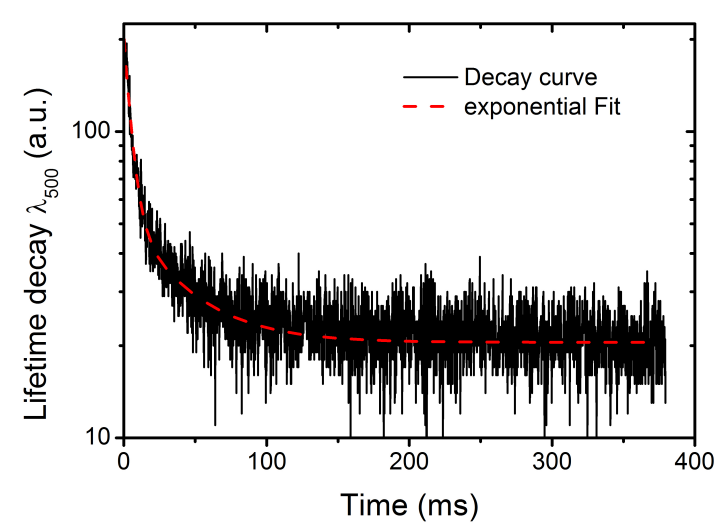

(a)

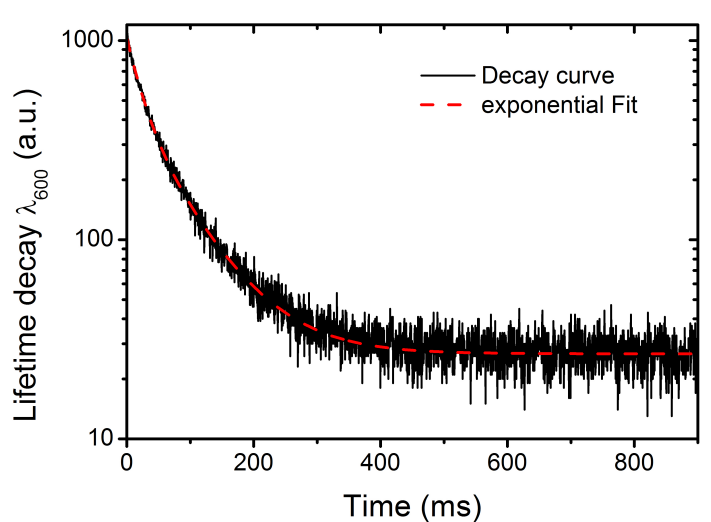

(b)

Figure 6.8: (a) PL lifetime decay of $1 \% \mathrm{Mn}$ doped $\mathrm{NaMgF}_{3}$ at $\lambda_{e m}=500 \mathrm{~nm}$ (solid curve) and (b) $\lambda_{e m}=600 \mathrm{~nm}$ (solid curve). The dashed curve is the exponential fit. The lifetime was measured at $\lambda_{e x}=465 \mathrm{~nm}$.

The average lifetime of the distorted site for the $\mathrm{NaMgF}_{3}$ bulk materials was $13 \pm 1.3 \mathrm{~ms}$ for both concentrations. This is similar to the lifetimes calculated for the distorted site in the nanoparticles. The regular site shows a lifetime as high as $98 \pm 10 \mathrm{~ms}$ for the $0.5 \%$ doped sample for the bulk materials, which is higher than calculated for the nanoparticles. The lower lifetime of the regular site in the nanoparticles means that this site is more distorted and transitions are more allowed. 


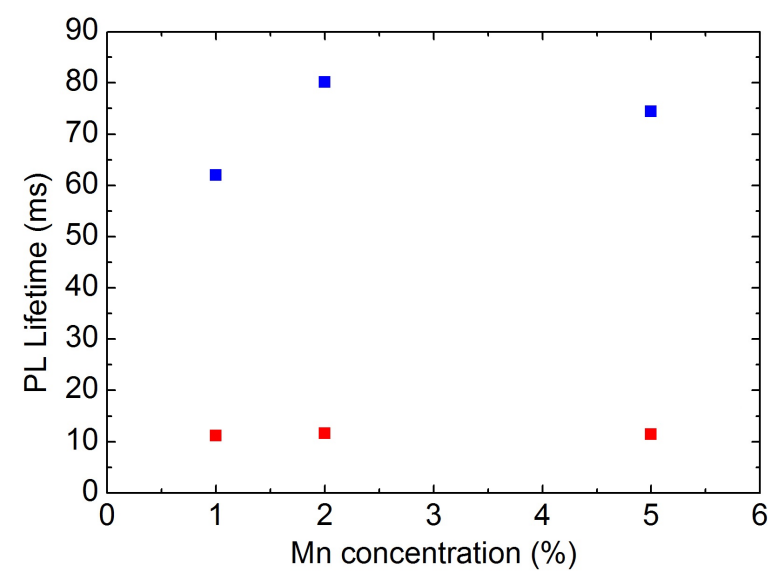

Figure 6.9: Average PL lifetime for the distorted $\mathrm{Mn}^{2+}$ site $\left(\lambda_{e m}=500 \mathrm{~nm}\right.$ red squares $)$ and the regular $\mathrm{Mn}^{2+}$ site $\left(\lambda_{e m}=600 \mathrm{~nm}\right.$ blue squares) doped $\mathrm{NaMgF}_{3}$ nanoparticles versus the rare earth concentration.

Table 6.3: Summary of different measured PL lifetimes, $\tau_{\text {ave }}$, for $\mathrm{NaMgF}_{3}$ doped with different concentrations of Mn.

\begin{tabular}{|l|l|}
\hline Concentration $(\%)$ & $\tau_{\text {ave }}(\mathrm{ms})$ \\
\hline \hline & $11.1 \pm 1.1(\mathrm{em} @ 500 \mathrm{~nm})$ \\
& $62.0 \pm 6.2(\mathrm{em} @ 600 \mathrm{~nm})$ \\
\hline & $11.6 \pm 1.2(\mathrm{em} @ 500 \mathrm{~nm})$ \\
2 & $80 \pm 8(\mathrm{em} @ 600 \mathrm{~nm})$ \\
\hline & $11.4 \pm 1.1(\mathrm{em} @ 500 \mathrm{~nm})$ \\
5 & $74 \pm 7(\mathrm{em} @ 600 \mathrm{~nm})$ \\
\hline
\end{tabular}




\subsection{Thermoluminescence measurements}

\subsubsection{Eu doped $\mathrm{NaMgF}_{3}$ nanoparticles}

The Eu doped $\mathrm{NaMgF}_{3}$ nanoparticles hardly show any TL. Therefore, the TL of the 0.5 $\%$ and the $5 \%$ doped samples were only plotted in Fig. 6.10 $(\mathrm{a}+\mathrm{b})$. The samples were irradiated for $20 \mathrm{~min}$ with an X-ray generator operating at $40 \mathrm{kV}$ and $40 \mathrm{~mA}$ using an Aluminium filter. Afterwards, the samples were heated up to $350{ }^{\circ} \mathrm{C}$ with a heating rate of $1 \mathrm{~K} / \mathrm{s}$ and glow curve data was collected using a PMT. The PMT could not detect the origin of the TL transition. Glow curve peaks were fitted using first order kinetics glow curve deconvolution. The glow curve peaks are listed in Tab. 6.4.

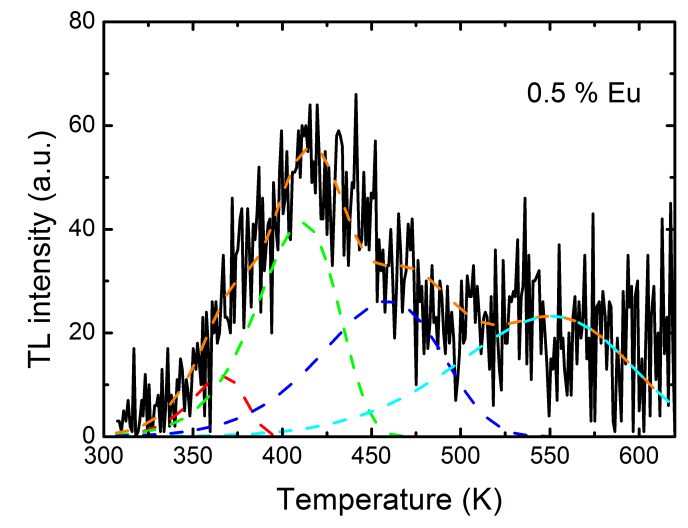

(a)

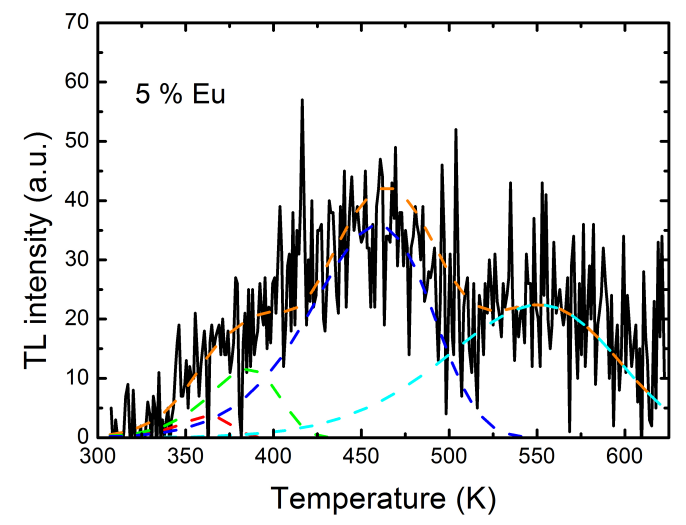

(b)

Figure 6.10: TL glow curves for $\mathrm{NaMgF}_{3}$ doped with $0.5 \%$ (a) and $5 \%$ (b) Eu after 20 min irradiation with the X-ray generator operating at $40 \mathrm{kV}$ and $40 \mathrm{~mA}$ (solid curves). The TL was detected with the PMT. The fitted peaks were obtained using first order kinetics glow curve deconvolution (dashed curves).

The values for the $5 \% \mathrm{Eu}$ doped sample compare to the values obtained for the bulk materials (see Tab. 5.5). There is only an additional high temperature peak at $\sim 551 \mathrm{~K}$ which was not observed for the bulk materials. The first peak centred at $363 \mathrm{~K}$ has the 
highest TL intensity in the bulk samples but it shows a low intensity for the nanoparticles. The peak intensity for the peak at $386 \mathrm{~K}$ for the $5 \% \mathrm{Eu}$ doped nanoparticle also has a lower intensity (Fig. 6.4(b)) compared to the bulk samples (see Fig. 5.8). The peak intensities of the TL glow curve peaks centred at $459 \mathrm{~K}$ and $551 \mathrm{~K}$ are similar for both concentrations of $\mathrm{Eu}$ in the nanoparticles. The glow curve peak at $363 \mathrm{~K}$ has the lowest TL intensity for both Eu doped samples. The $2^{\text {nd }}$ glow curve peak shifts towards lower temperatures for the $5 \% \mathrm{Eu}$ doped sample, the peak intensity also increases compared to the $0.5 \%$ sample. Although the TL measurements were detected with the PMT and it could not be distinguished if the TL signal arises from the $\mathrm{Eu}^{2+}$ ions or the $\mathrm{Eu}^{3+}$ ones. The activation energies obtained from the nanoparticles were similar to the bulk sample. Therefore, it can be assumed that the TL signal is due to same traps from the $\mathrm{Eu}^{2+}$ emission. Potential additional peaks above $620 \mathrm{~K}$ could not be detected due to the temperature limitation of the equipment used.

Table 6.4: The activation energies of the individual glow curve peaks for the $\mathrm{NaMgF}_{3}: 0.5$ $\%$ and $5 \%$ Eu sample obtained by first order kinetics glow curve deconvolution. The top line is the activation energy for the glow curve.

\begin{tabular}{|l|l|l|l|l|}
\hline & $0.76 \pm 0.08 \mathrm{eV}$ & $0.61 \pm 0.06 \mathrm{eV}$ & $0.50 \pm 0.05 \mathrm{eV}$ & $0.50 \pm 0.05 \mathrm{eV}$ \\
\hline \hline $0.5 \%$ & $363 \mathrm{~K}$ & $411 \mathrm{~K}$ & $459 \mathrm{~K}$ & $551 \mathrm{~K}$ \\
peak height & 10 & 40 & 30 & 200 \\
\hline $5 \%$ & $363 \mathrm{~K}$ & $386 \mathrm{~K}$ & $459 \mathrm{~K}$ & $551 \mathrm{~K}$ \\
peak height & 10 & 10 & 40 & 20 \\
\hline
\end{tabular}

\subsubsection{Mn doped $\mathrm{NaMgF}_{3}$ nanoparticles}

The TL glow curves for the Mn doped samples with different concentrations are plotted in Fig. 6.11 and activation energies for the individual glow curve peaks are listed in Tab. 6.5. The TL glow curve peaks were calculated with the first order kinetics glow curve 
deconvolution. The TL data was collected using a PMT after an irradiation of 20 min with a X-ray generator $(40 \mathrm{kV}, 40 \mathrm{~mA})$ using an Aluminium filter at a heating rate of 1 $\mathrm{K} / \mathrm{s}$.

The nanoparticles show different activation energies and their peak maximums are found at different temperatures compared to the bulk materials. The main peaks for the bulk materials are observed for higher temperatures, where the nanoparticles have their main peaks at lower temperatures up to $\sim 500 \mathrm{~K}$. The high temperature glow curve peak shifts from $\sim 516 \mathrm{~K}$ to $\sim 535 \mathrm{~K}$ with increasing Mn concentration of the sample. The other peak maxima are constant within a range of $4 \mathrm{~K}$. The low temperature peak centred at $354 \mathrm{~K}$ shows an activation energy of $0.76 \mathrm{eV}$. For the $1 \% \mathrm{Mn}$ doped nanoparticles the main three peaks are found at $381 \mathrm{~K}, 401 \mathrm{~K}$ and $450 \mathrm{~K}$, with respectively an activation energies of $0.6 \mathrm{eV}, 0.54 \mathrm{eV}$ and $0.5 \mathrm{eV}$. For the $5 \%$ doped sample the activation energy was $0.54 \mathrm{eV}$ and $0.5 \mathrm{eV}$ for peaks centred at $401 \mathrm{~K}$ and $464 \mathrm{~K}$, respectively. For the $1 \%$ doped sample, additional TL measurements were performed after irradiating the sample with different doses to investigate the dependence of the TL intensity on the initial dose (Fig. 6.11(d)). Therefore, the sample was irradiated with a dose of 1056 Gy and TL was measured. After this, the sample was irradiated again with a dose of 3168 Gy, which gives a total dose of 4224 Gy. TL was measured again. Then, the sample was irradiated again with a dose of 1056, which leads to a total dose of 5280 Gy. TL measurements were performed again. The TL glow curve shows a different shape after receiving a dose of 1056 Gy compared to receiving a total dose of $5280 \mathrm{~Gy}$. The reason for this is that the probability of trapping changes after receiving a higher dose. For higher doses the filling of additional traps might increase resulting in a less sharp peak at $375 \mathrm{~K}$ when receiving a total dose of 4224 Gy. Fig. 6.11(d) shows the TL intensity decreases when receiving a total dose of 5280 Gy compared to TL measurements when receiving a dose of 1056 Gy. No significant change in activation energies or temperatures after receiving different doses was observed. Only the concentration of the dopant has an influence on the temperature shift of the glow curves peaks. 


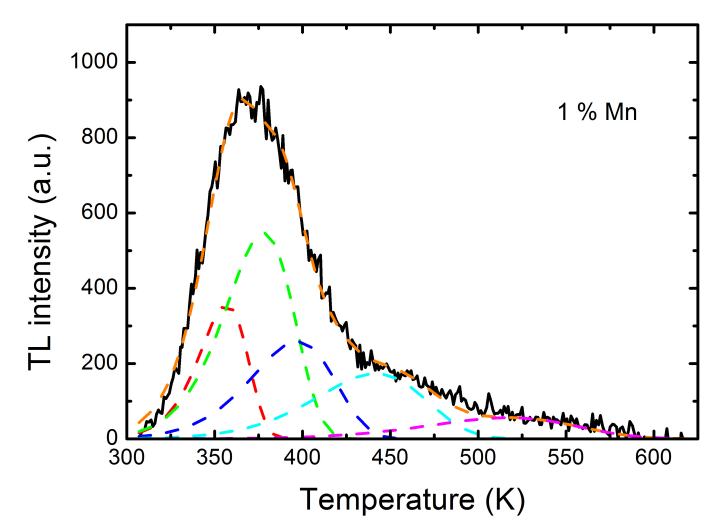

(a)

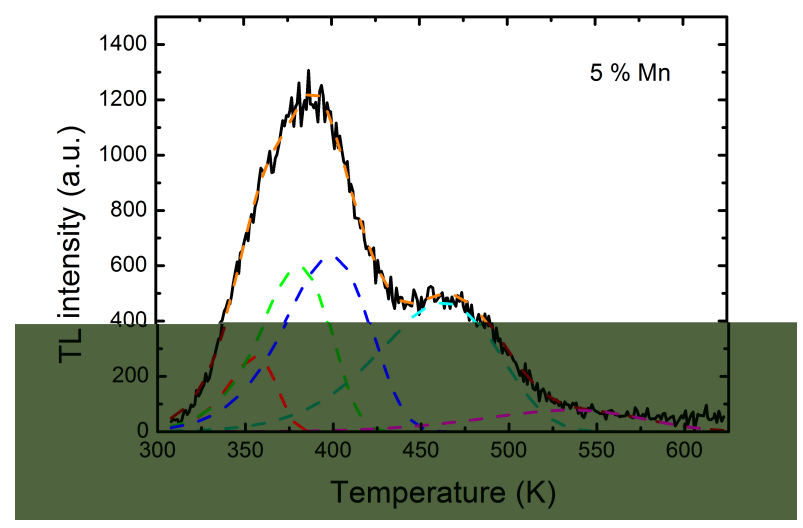

(c)

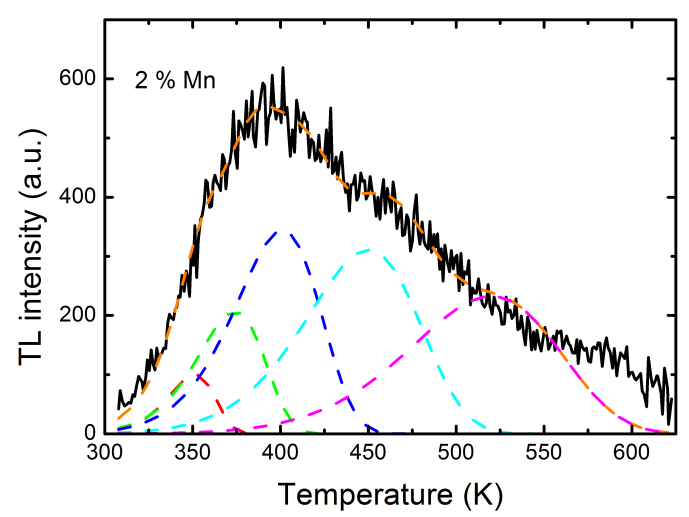

(b)

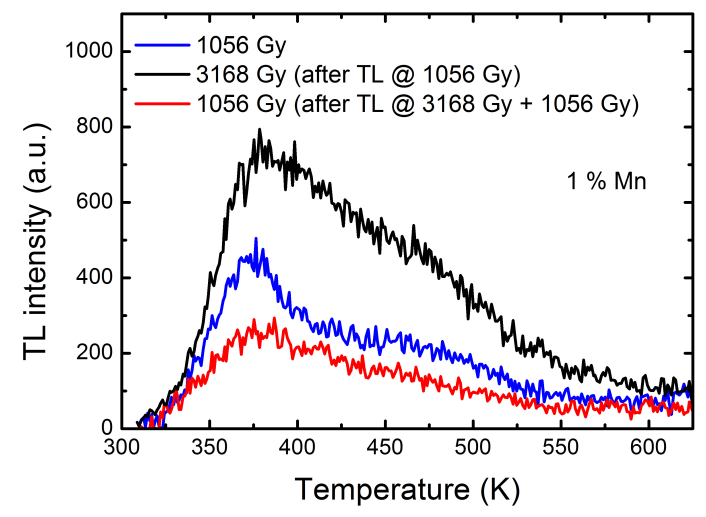

(d)

Figure 6.11: TL glow curves for $\mathrm{NaMgF}_{3}$ doped with 1\% Mn (a), $2 \% \mathrm{Mn}$ (b), $5 \% \mathrm{Mn}$ (c) after 20 min irradiation and for $1 \% \mathrm{Mn}$ after $10 \mathrm{~min}, 30 \mathrm{~min}$ and again 10 min (d) irradiation with the X-ray generator operating at $40 \mathrm{kV}$ and $40 \mathrm{~mA}$ (solid curves). The TL was detected with the PMT. The fitted peaks were obtained using first order kinetics glow curve deconvolution (dashed curves).

The intense glow curve peaks are found at $380 \mathrm{~K}, 403 \mathrm{~K}$ and $450 \mathrm{~K}$. The peak at temperatures above $510 \mathrm{~K}$ show a lower TL glow curve height for the $1 \%$ and $5 \%$ doped samples. In comparison with the Mn doped bulk materials, the TL glow curve peak at a temperature of $\sim 464 \mathrm{~K}$ with an activation energy of $0.5 \mathrm{eV}$ was similar (see Tab. 5.6). These values compare well with the values obtained for the Eu doped $\mathrm{NaMgF}_{3}$ bulk material (Tab. 5.5 
and Tab. 6.4). It is likely that these defects arise from the same defects. The TL signal intensity was too low to be detected with the CCD detector but as the temperatures and the activation energies for the Mn doped nanoparticles are comparable to the Mn doped bulk materials it is more likely that the TL signal arises from the $600 \mathrm{~nm}$ emission. Potential additional peaks above $620 \mathrm{~K}$ could not be detected due to the temperature limitation of the equipment used.

Table 6.5: The activation energies of the individual glow curve peaks for $\mathrm{NaMgF}_{3}$ doped with different concentration of $\mathrm{Mn}^{2+}$ obtained by first order kinetics glow curve deconvolution. The top line is the activation energy for the different peaks for the glow curves.

\begin{tabular}{|l|l|l|l|l|l|}
\hline & $\begin{array}{l}0.76 \pm 0.08 \\
\mathrm{eV}\end{array}$ & $\begin{array}{l}0.60 \pm 0.06 \\
\mathrm{eV}\end{array}$ & $\begin{array}{l}0.54 \pm 0.05 \\
\mathrm{eV}\end{array}$ & $\begin{array}{l}0.50 \pm 0.05 \\
\mathrm{eV}\end{array}$ & $\begin{array}{l}0.50 \pm 0.05 \\
\mathrm{eV}\end{array}$ \\
\hline \hline $\begin{array}{l}1 \%(10 \mathrm{~min}) \\
\text { peak height }\end{array}$ & $\begin{array}{l}359 \mathrm{~K} \\
100\end{array}$ & $\begin{array}{l}379 \mathrm{~K} \\
280\end{array}$ & $\begin{array}{l}401 \mathrm{~K} \\
130\end{array}$ & $\begin{array}{l}449 \mathrm{~K} \\
180\end{array}$ & $\begin{array}{l}516 \mathrm{~K} \\
140\end{array}$ \\
\hline $\begin{array}{l}1 \% \text { (20 min) } \\
\text { peak height }\end{array}$ & $357 \mathrm{~K}$ & $376 \mathrm{~K}$ & $404 \mathrm{~K}$ & $449 \mathrm{~K}$ & $519 \mathrm{~K}$ \\
$1 \%$ (30 min) & 350 & 550 & 260 & 170 & 60 \\
peak height & $357 \mathrm{~K}$ & $373 \mathrm{~K}$ & $403 \mathrm{~K}$ & $449 \mathrm{~K}$ & $516 \mathrm{~K}$ \\
\hline $1 \%$ (10 min-repeat) & $357 \mathrm{~K}$ & $373 \mathrm{~K}$ & $403 \mathrm{~K}$ & $449 \mathrm{~K}$ & $516 \mathrm{~K}$ \\
peak height & 60 & 130 & 130 & 100 & 80 \\
\hline $2 \%(20 \mathrm{~min})$ & $351 \mathrm{~K}$ & $374 \mathrm{~K}$ & $401 \mathrm{~K}$ & $450 \mathrm{~K}$ & $520 \mathrm{~K}$ \\
peak height & 100 & 200 & 340 & 310 & 230 \\
\hline $5 \%$ (20 min) & $356 \mathrm{~K}$ & $381 \mathrm{~K}$ & $400 \mathrm{~K}$ & $464 \mathrm{~K}$ & $535 \mathrm{~K}$ \\
peak height & 270 & 600 & 630 & 470 & 80 \\
\hline
\end{tabular}




\subsection{Radioluminescence and temperature dependent RL measurements}

Radioluminescence measurements were performed on pressed pellets. The temperature dependent RL measurements were performed on the pulverised samples, immersed with isopropanol and dispersed on a ceramic heat element to control heating.

\subsubsection{Radioluminescence measurements}

\subsubsection{Eu doped $\mathrm{NaMgF}_{3}$ nanoparticles}

In Fig. 6.12 to 6.14, the RL measurements of the Eu doped $\mathrm{NaMgF}_{3}$ nanoparticles are plotted. RL was measured using a CCD while irradiating the sample with an X-ray generator. For lower doses the sample was irradiated with an ${ }^{241} \mathrm{Am}$ source and the data was collected using a PMT (solid curve in Fig. 6.14(b)). The RL arises mainly from $\mathrm{Eu}^{2+}$ and $\mathrm{Eu}^{3+}$ emission and their energies are the same as those observed in the PL spectra. There is no shift of RL emission wavelengths in the spectra for transitions of the two ions after heavy irradiation. The RL intensity against the dose shows the same behaviour for all different concentrations of $\mathrm{Eu} . \mathrm{I}_{R L}$ decreases at a slower rate for the $\mathrm{Eu}^{3+}$ ion compared to the $\mathrm{Eu}^{2+}$ ion. It was calculated that the integrated $\mathrm{RL}$ intensity of the $\mathrm{Eu}^{2+}$ ion decreases by $28.5 \%$ at $1 \mathrm{kGy}$ and by $77.4 \%$ at $12.7 \mathrm{kGy}$ for the $0.1 \%$ doped sample. $\mathrm{I}_{R L}$ decreases by $2.6 \% 1 \mathrm{kGy}$ and by $8 \%$ at $12.7 \mathrm{kGy}$ for the $\mathrm{Eu}^{3+}$ ion (Fig. 6.12(a+b)). A change in the ratio $\mathrm{I}_{\mathrm{Eu}^{3+}} / \mathrm{I}_{\mathrm{Eu}^{2+}}$ from 1.3:1 at 35 Gy to 5.3:1 at $12.7 \mathrm{kGy}$ was observed. 


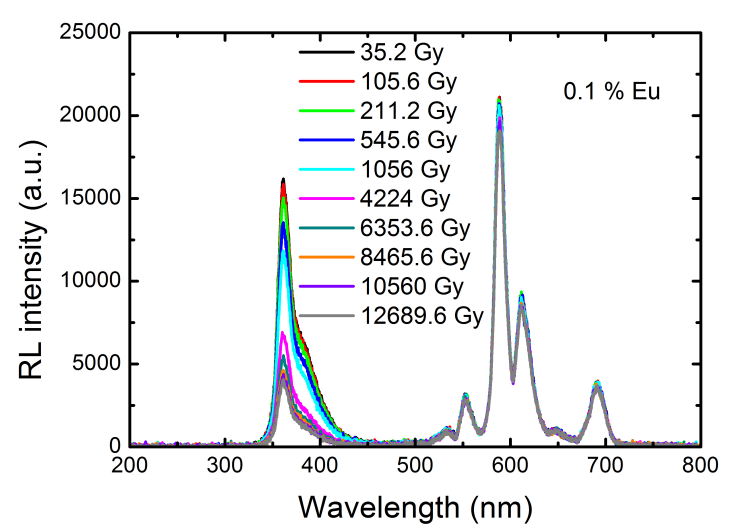

(a)

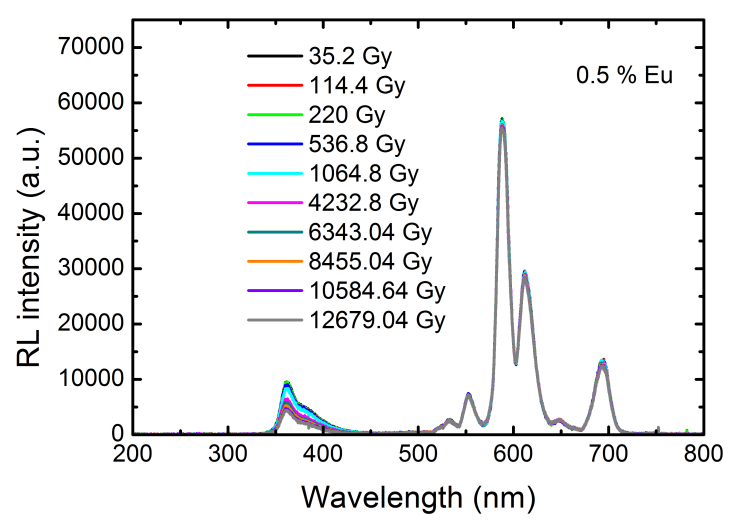

(c)

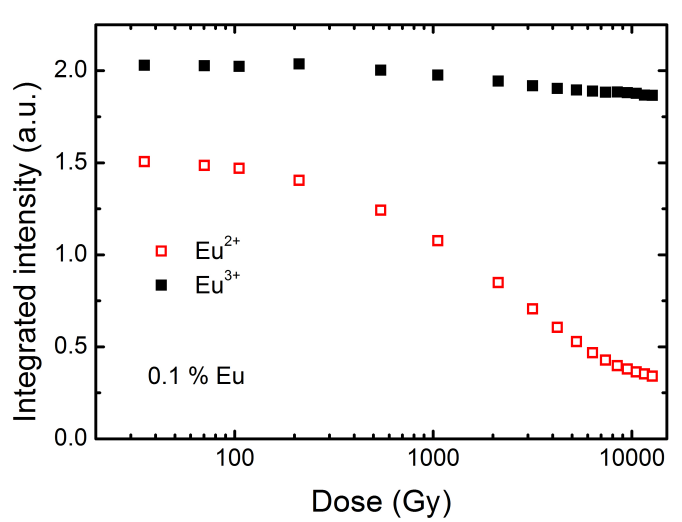

(b)

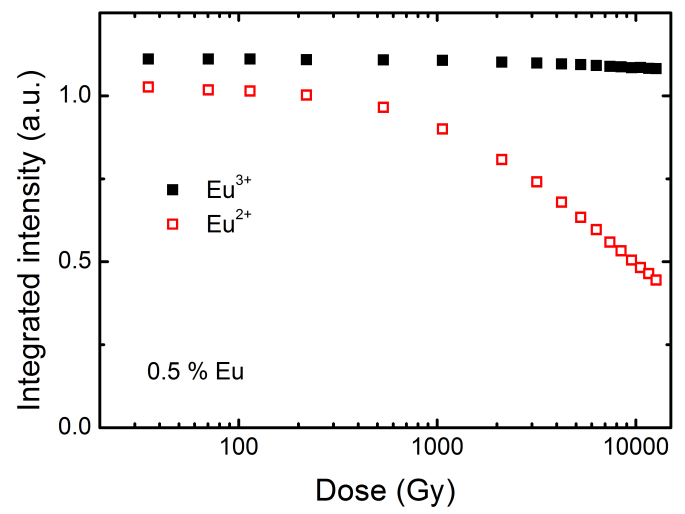

(d)

Figure 6.12: Radioluminescence spectra for $\mathrm{NaMgF}_{3}$ nanoparticles doped with $0.1 \% \mathrm{Eu}$ (a) and $0.5 \% \mathrm{Eu}(\mathrm{c})$ for different doses and the integrated RL spectra as a function of dose during continuous X-ray irradiation for $0.1 \% \mathrm{Eu}(\mathrm{b})$ and 0.5 $\% \mathrm{Eu}(\mathrm{d})$ doped samples $\left(\mathrm{Eu}^{2+}\right.$ open squares and $\mathrm{Eu}^{3+}$ filled squares).

For the $0.5 \%$ sample, the integrated intensity $\left(\mathrm{I}_{R L}\right)$ decreases by $12.3 \%$ at $1 \mathrm{kGy}$ and by $56.7 \%$ at $12.7 \mathrm{kGy}$ for the $\mathrm{Eu}^{2+}$ ion. The RL intensity decreases by $0.3 \% 1 \mathrm{kGy}$ and by $2.6 \%$ at $12.7 \mathrm{kGy}$ for the $\mathrm{Eu}^{3+}$ ion (Fig. 6.12 $(\mathrm{c}+\mathrm{d})$ ). A change $\mathrm{I}_{E u^{3+}} / \mathrm{I}_{E u^{2+}}$ from 5.8:1 at 35 Gy to $18: 1$ at 12.7 kGy was calculated. 


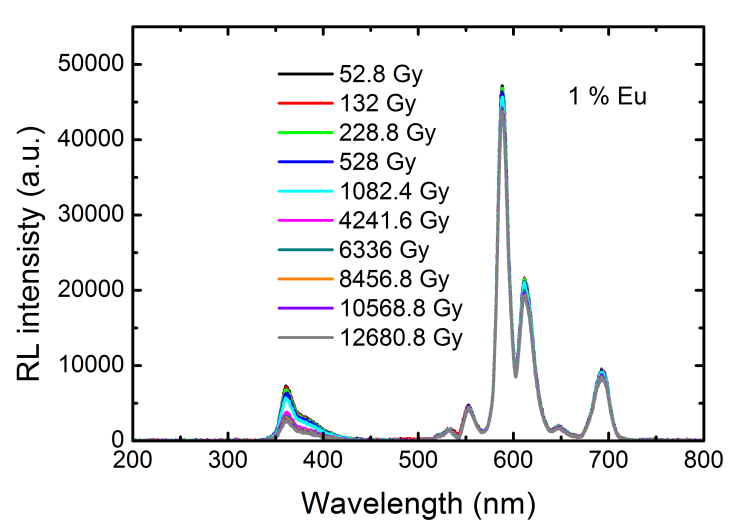

(a)

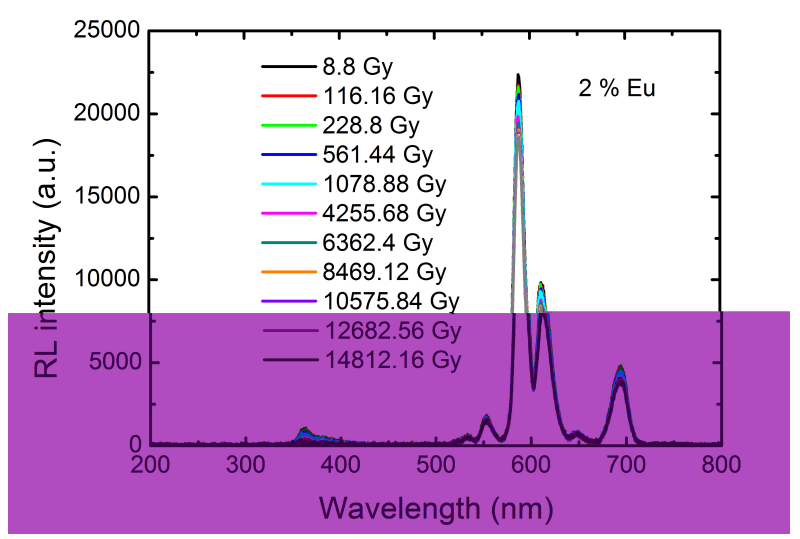

(c)

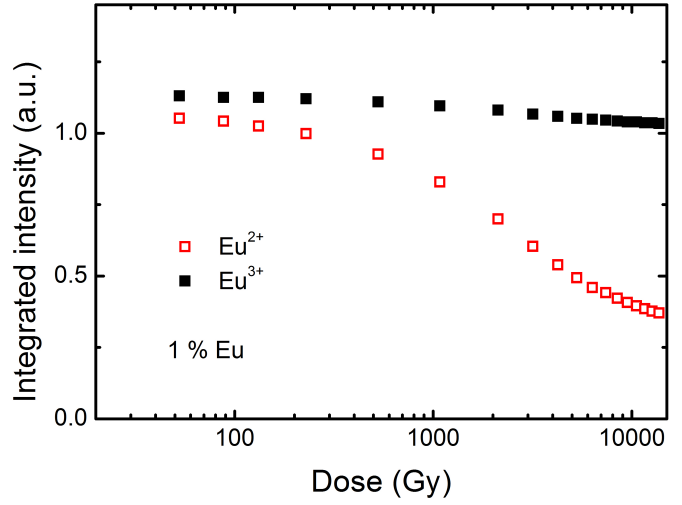

(b)

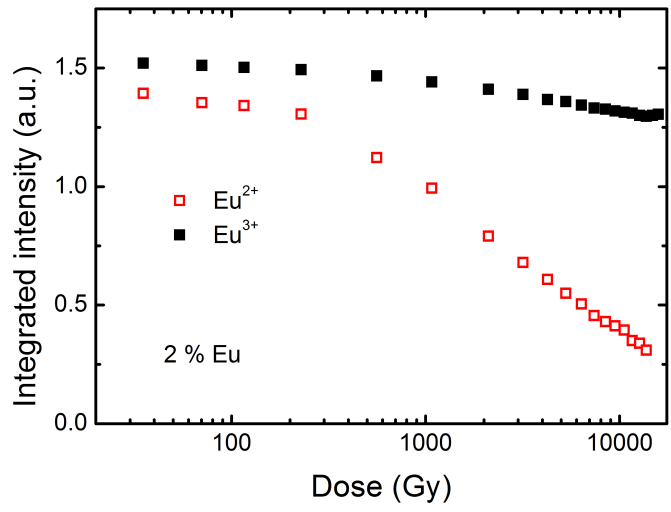

(d)

Figure 6.13: Radioluminescence spectra for $\mathrm{NaMgF}_{3}$ nanoparticles doped with $1 \% \mathrm{Eu}$ (a) and $2 \% \mathrm{Eu}(\mathrm{c})$ for different doses and the integrated RL spectra as a function of dose during continuous X-ray irradiation for $1 \% \mathrm{Eu}(\mathrm{b})$ and $2 \% \mathrm{Eu}(\mathrm{d})$ doped samples $\left(\mathrm{Eu}^{2+}\right.$ open squares and $\mathrm{Eu}^{3+}$ filled squares).

$\mathrm{I}_{R L}$ decreases by $21.2 \%$ at $1 \mathrm{kGy}$ and by $64.8 \%$ at $12.7 \mathrm{kGy}$ for the $\mathrm{Eu}^{2+}$ ion. The RL intensity decreases by $3.1 \% 1 \mathrm{kGy}$ and by $8.6 \%$ at $12.7 \mathrm{kGy}$ for the $\mathrm{Eu}^{3+}$ ion for $\mathrm{NaMgF}_{3}$ : $1 \% \mathrm{Eu}$ (Fig. 6.13 $(\mathrm{a}+\mathrm{b}))$. In this case $\mathrm{I}_{E u^{3+}} / \mathrm{I}_{E u^{2+}}$ changes from 6.3:1 at 52 Gy to $16: 1$ at $12.7 \mathrm{kGy}$. The $2 \%$ doped sample shows a decrease by $28.7 \%$ at $1 \mathrm{kGy}$ and by $77.8 \%$ at $12.7 \mathrm{kGy}$ for the $\mathrm{Eu}^{2+}$ ion. $\mathrm{I}_{R L}$ decreases by $5.2 \% 1 \mathrm{kGy}$ and by $14.7 \%$ at $12.7 \mathrm{kGy}$ for the $\mathrm{Eu}^{3+}$ ion (Fig. 6.13 $(\mathrm{c}+\mathrm{d})$ ). A ratio of $21: 1$ for $\mathrm{I}_{E u^{3+}} / \mathrm{I}_{E u^{2+}}$ at $35 \mathrm{~Gy}$ and $75: 1$ at 12.7 
kGy was estimated.

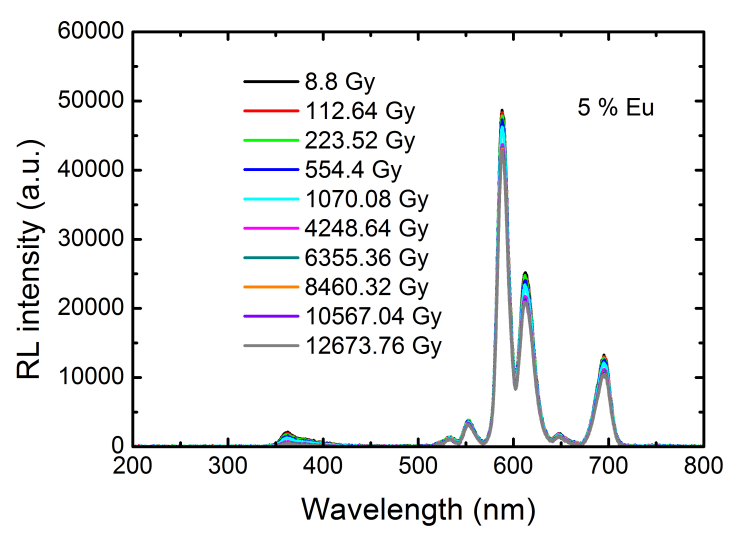

(a)

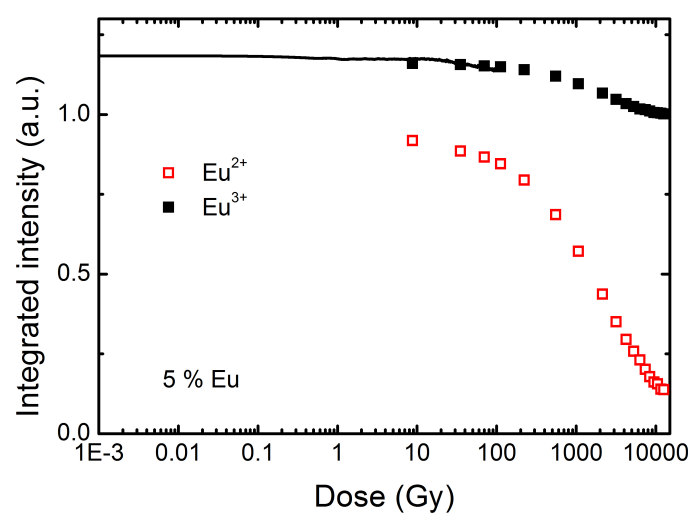

(b)

Figure 6.14: Radioluminescence spectra for $\mathrm{NaMgF}_{3}$ nanoparticles doped with $5 \% \mathrm{Eu}$ (a) for different doses and the integrated RL spectra as a function of dose during continuous X-ray irradiation for $5 \% \mathrm{Eu}$ (b) doped samples $\left(\mathrm{Eu}^{2+}\right.$ open squares and $\mathrm{Eu}^{3+}$ filled squares, solid line ${ }^{241} \mathrm{Am}$ irradiation). The $\gamma$-ray RL was scaled to match the X-ray RL.

The highest decrease of the integrated RL intensity of the $\mathrm{Eu}^{2+}$ was observed for the $5 \%$ doped sample. The intensity decreases by $35.5 \%$ at $1 \mathrm{kGy}$ and by $84.4 \%$ at $12.7 \mathrm{kGy}$ for the $\mathrm{Eu}^{2+}$ ion. The RL intensity decreases by $5.6 \% 1 \mathrm{kGy}$ and by $13.6 \%$ at $12.7 \mathrm{kGy}$ for the $\mathrm{Eu}^{3+}$ ion (Fig. 6.14 $(\mathrm{a}+\mathrm{b})$ ). The $\mathrm{I}_{\mathrm{Eu}^{3+}} / \mathrm{I}_{\mathrm{Eu} u^{2+}}$ ratio changes from 23:1 at $35 \mathrm{~Gy}$ to $125: 1$ at $12.7 \mathrm{kGy}$. The decrease in $\mathrm{I}_{R L}$ for the $\mathrm{Eu}^{3+}$ at high doses is likely to occur through the generation of defects that act as non-radiative decay centres. It is not clear why the RL integrated intensity increases at a faster rate for $\mathrm{Eu}^{2+}[10,11]$. For example, it decreased by $84.4 \%$ at $12.7 \mathrm{kGy}$ for the $5 \%$ sample. It might be because radiation induced defects preferentially occur near $\mathrm{Eu}^{2+}$ sites leading to more quenching of the $\mathrm{Eu}^{2+}$ $\mathrm{RL}$ compared with the $\mathrm{Eu}^{3+}$ intensity. It is apparent in Fig. 6.14(b) that the $\mathrm{Eu}^{3+} \mathrm{RL}$ is dose-independent down to at least $10 \mathrm{mGy}$ for low doses for the $5 \% \mathrm{Eu}$ sample. This was measured with the ${ }^{241} \mathrm{Am}$ source, which emits $\gamma$-rays. The decrease of the integrated RL 
signal might be due to the creation of additional point defects, resulting in non-radiative decay [10]. The ratio of $\mathrm{I}_{7} F_{2} / \mathrm{I}_{7} F_{1}$ does not change after heavy irradiation and it is around 1:1.7 for all doses and concentrations. In the PL spectra, the ratio of $\mathrm{I}_{7} F_{2} / \mathrm{I}_{7 F_{1}}$ is dependent on the $\mathrm{Eu}^{3+}$ concentration. It was calculated as 1:1.6 for the $0.1 \%$ sample, 1:1.3 for the 1 $\%$ sample and decreases to 1:1.2 for the $5 \%$ sample. The average crystal environment of $\mathrm{Eu}^{3+}$ seem to be more distorted with increasing $\mathrm{Eu}^{3+}$ concentration.

The $5 \%$ doped sample shows the biggest change in the ratio of the intensity of $\mathrm{Eu}^{3+} / \mathrm{Eu}^{2+}$ in the RL spectra and it changes from 23:1 to $125: 1$. This decrease of $\mathrm{I}_{R L}$ for the $\mathrm{Eu}^{2+}$ ion might be due to the conversion from $\mathrm{Eu}^{2+}$ to $\mathrm{Eu}^{3+}$, which was reported for the bulk materials (see chapter 5). The ratio $\mathrm{Eu}^{2+} / \mathrm{Eu}^{3+}$ is $1.8: 1$ in the PL spectrum for the $5 \%$ doped sample. There is no RL transition detected from the unknown defect between 400 $\mathrm{nm}$ to $500 \mathrm{~nm}$ but this was observed in the RL spectrum for the bulk materials (see Fig. $5.13(\mathrm{a}))$.

In a recent paper, Williams et al. reported the $\mathrm{RL}$ behaviour of $\mathrm{Eu}^{2+}$ and $\mathrm{Eu}^{3+}$ for doses up to $30 \mathrm{kGy}$. They showed that the integrated RL intensity of $\mathrm{Eu}^{2+}$ and $\mathrm{Eu}^{3+}$ for a 1 $\%$ Eu doped sample decreases up to $\sim 10 \mathrm{kGy}$ and decreases at a slower rate above $\sim 10$ kGy, which is shown in Fig. 6.15. The size of the nanoparticles was $57 \mathrm{~nm}$ for the $1 \%$ Eu doped sample, which were investigated in the paper [10]. The size of the nanoparticles researched in this thesis were $25 \mathrm{~nm}$. 


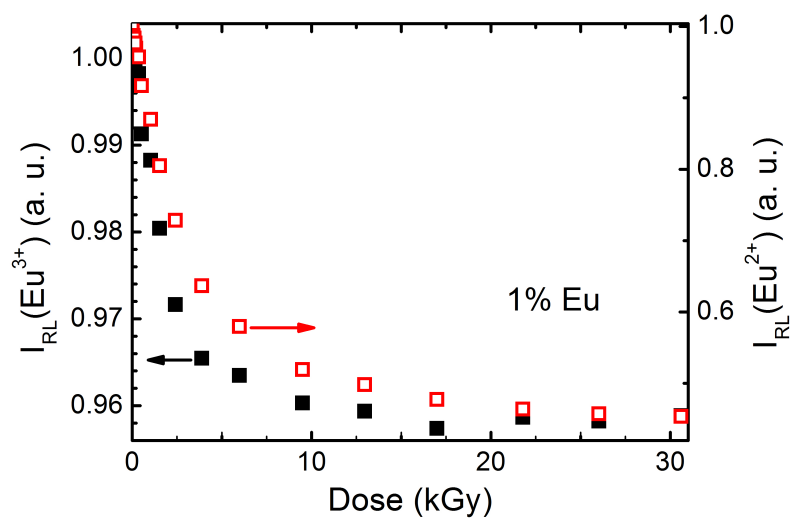

Figure 6.15: The integrated $\mathrm{Eu}^{3+}$ (filled squares, left axis) and $\mathrm{Eu}^{2+}$ (open squares, right axis) RL spectra as a function of radiation dose for $\mathrm{NaMgF}_{3}: 1 \% \mathrm{Eu}$ nanoparticles with a crystal size of $57 \mathrm{~nm}[10]$.

\subsubsection{2 $\mathrm{Mn}$ doped $\mathrm{NaMgF}_{3}$ nanoparticles}

The following Fig. 6.16 and 6.17 are showing the RL spectra against dose for $\mathrm{NaMgF}_{3}$ doped with different concentrations of Mn.

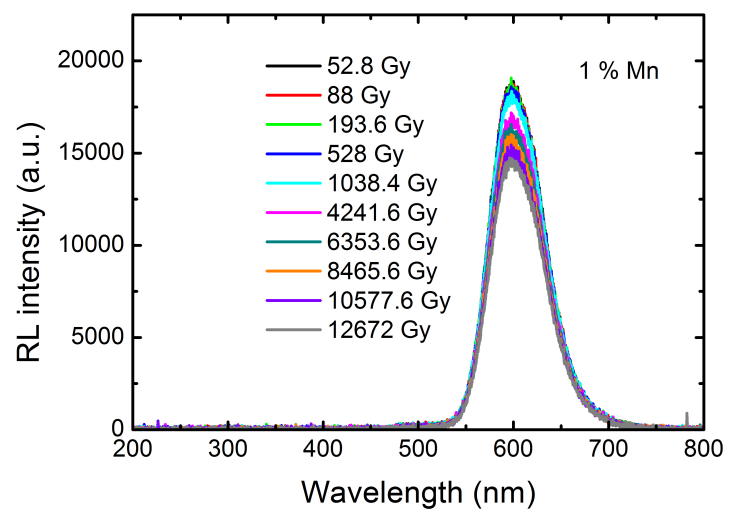

(a)

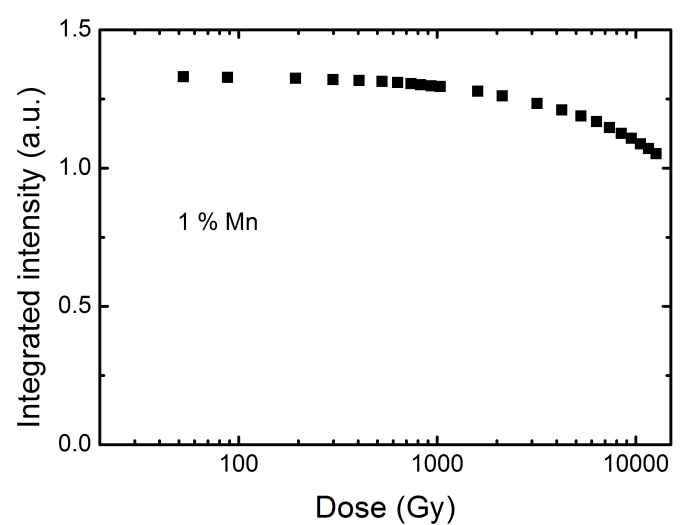

(b)

Figure 6.16: Radioluminescence spectra for $\mathrm{NaMgF}_{3}$ nanoparticles doped with $1 \% \mathrm{Mn}$ (a) for different doses and the integrated RL spectra as a function of dose during continuous X-ray irradiation for $1 \% \mathrm{Mn}$ (b) doped samples. 
For all of the nanoparticles, the RL of the distorted site centred at $500 \mathrm{~nm}$ was to weak and therefore, the dose dependence could not be calculated from the RL emission spectra. The strongest decrease of the integrated RL signal with increasing dose was observed for the $1 \% \mathrm{Mn}$ doped sample (Fig. 6.17(b)). The RL intensity for the regular site decreases by $2.7 \%$ at $1 \mathrm{kGy}$ and decreases until $20.9 \%$ at $12 \mathrm{kGy}$ (Fig. 6.17(b)).

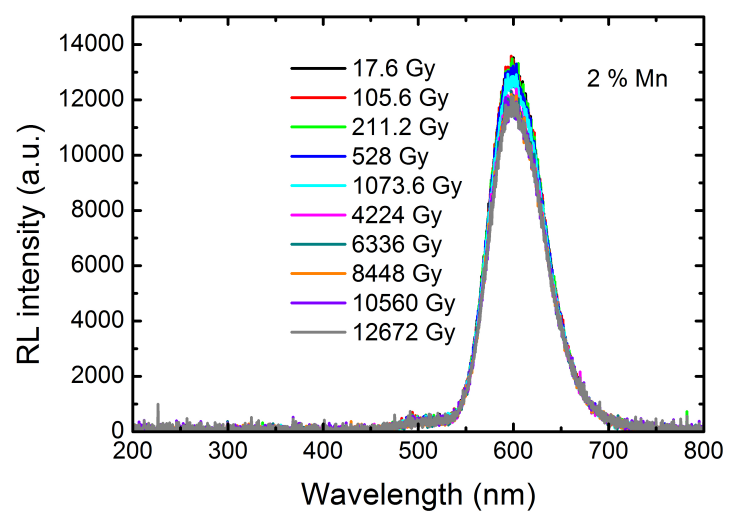

(a)

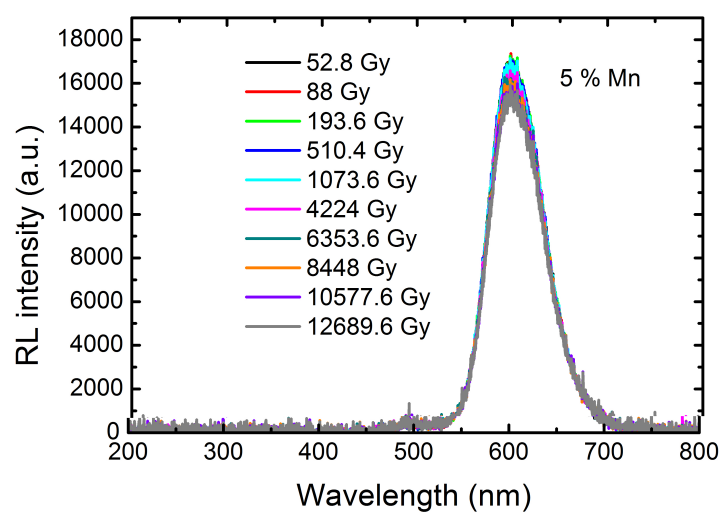

(c)

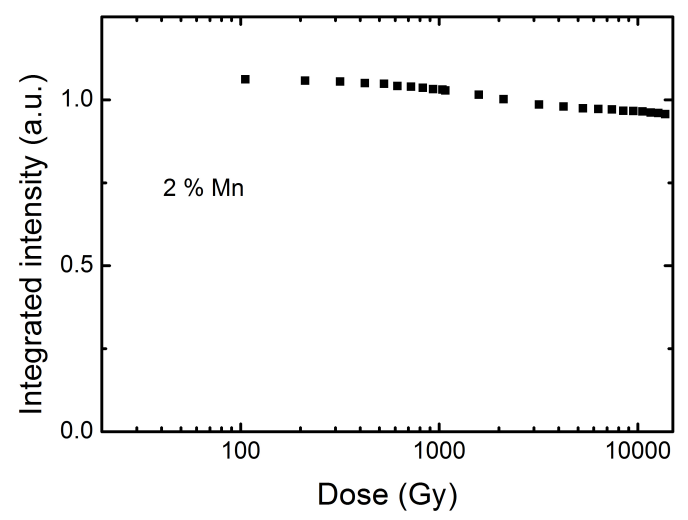

(b)

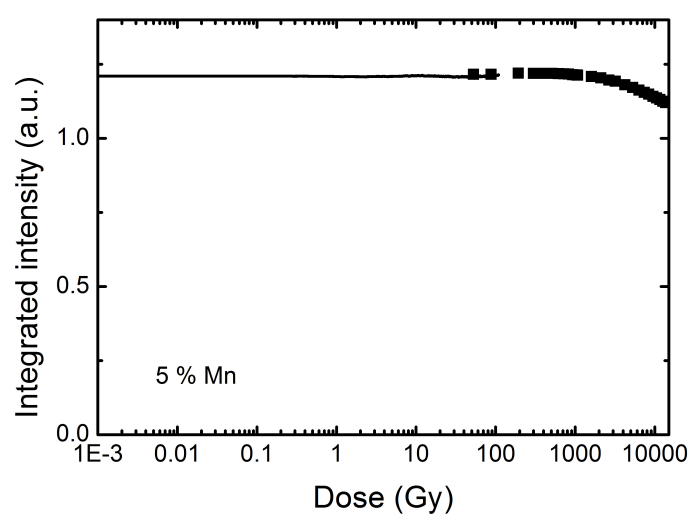

(d)

Figure 6.17: Radioluminescence spectra for $\mathrm{NaMgF}_{3}$ nanoparticles doped with $2 \% \mathrm{Mn}$ (a) and and $5 \% \mathrm{Mn}(\mathrm{c})$, for different doses and the integrated RL spectra as a function of dose during continuous X-ray irradiation for $2 \% \mathrm{Mn}(\mathrm{b})$, and $5 \%$ Mn (d) doped samples, solid line ${ }^{241}$ Am irradiation. The $\gamma$-ray RL was scaled to match the X-ray RL. 
A decrease by $2.9 \%$ at $1 \mathrm{kGy}$ and by $9.6 \%$ at $12 \mathrm{kGy}$ was calculated for the $2 \% \mathrm{Mn}$ doped nanoparticles. The lowest dose dependence of RL signal was observed at the $5 \%$ doped sample. The signal decreases by $0.2 \%$ at $1 \mathrm{kGy}$ and by $7.52 \%$ at $12 \mathrm{kGy}$. Fig. 6.17(d) shows RL measurements down to $\sim 10 \mathrm{mGy}$ for the $5 \% \mathrm{Mn}$ doped sample. These measurements were performed with an ${ }^{241} \mathrm{Am}$ nuclide that emits $\gamma$-rays.

Fig. 6.18 shows long-term RL measurements of $\mathrm{NaMgF}_{3}$ doped with $1 \% \mathrm{Mn}$, which were measured up to a dose of $20 \mathrm{kGy}$. These measurements were performed by Williams et al. for nanoparticles with a bigger crystal size $(53 \mathrm{~nm})$ [10]. The size of the nanoparticles researched in this thesis was $25 \mathrm{~nm}$. Williams et al. reported, the integrated RL intensity decreases up to a dose of $\sim 10 \mathrm{kGy}$ and $\mathrm{I}_{R L}$ saturates above $\sim 10 \mathrm{kGy}$. The RL signal for the Eu doped samples decreases with increasing dose (see Fig. 6.13). This indicates that the decreasing RL signal is caused by the creation of point defects with non-radiative recombination centres near the $\mathrm{Mn}^{2+}[10]$.

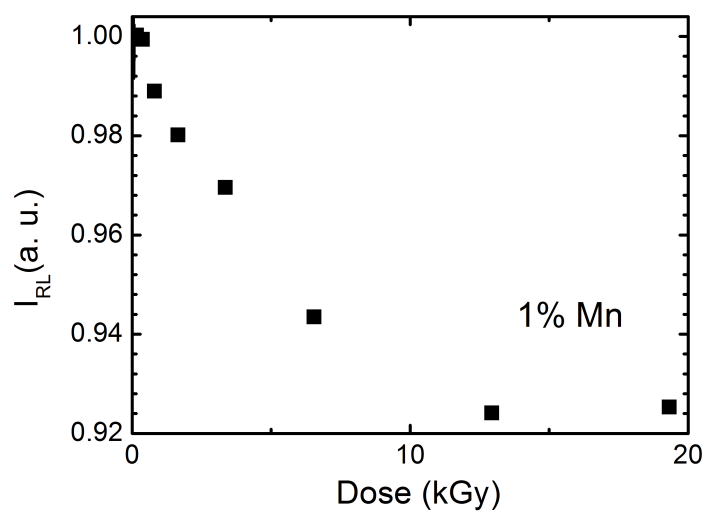

Figure 6.18: The integrated RL spectra as a function of radiation dose for $\mathrm{NaMgF}_{3}: 1 \%$ $\mathrm{Mn}^{2+}$ nanoparticles with a crystal size of $53 \mathrm{~nm}[10$. 


\subsubsection{Temperature dependent RL measurements}

\subsubsection{Eu doped $\mathrm{NaMgF}_{3}$ nanoparticles}

Temperature dependent RL measurements were performed with a custom-built TL setup employing pulverized samples, immersed in isopropanol and dispersed on a ceramic holder. The integration time for each measurement was $5 \mathrm{~s}$ and 10 measurements were taken at each temperature step. The sample was heated from $40{ }^{\circ} \mathrm{C}$ to $100{ }^{\circ} \mathrm{C}$ in $10{ }^{\circ} \mathrm{C}$ steps. At a certain temperature, the samples were thermally bleached with temperatures as high as $350{ }^{\circ} \mathrm{C}$. The sample received a total dose of $1.2 \mathrm{kGy}$ at the end of these measurements.

The temperature dependent RL spectra for the $0.1 \%$ and $5 \%$ Eu doped samples are shown in Fig. 6.19(a-d). In Fig. 6.19)(a) it was observed that the RL intensity is not decreasing until it reaches the initial measured RL intensity at $40{ }^{\circ} \mathrm{C}$. The intensity of the last measurement at $40{ }^{\circ} \mathrm{C}$ is smaller than the intensity at the first measurement at $40{ }^{\circ} \mathrm{C}$. For the $0.1 \%$ RL intensity increases by $0.2 \%$ compared to room temperature when the temperature is increased to $100{ }^{\circ} \mathrm{C}$, and decreases by $11.8 \%$ compared to $100{ }^{\circ} \mathrm{C}$ when decreasing the temperature to $40{ }^{\circ} \mathrm{C}$ leading to a total difference of $11.6 \%$. The integrated $\mathrm{RL}$ intensity for the $\mathrm{Eu}^{2+}$ ion decreases by $2.8 \%$ compared to room temperature when the temperature is increased to $100{ }^{\circ} \mathrm{C}$ and decreases by $15.1 \%$ compared to $100{ }^{\circ} \mathrm{C}$ when decreasing the temperature to $40{ }^{\circ} \mathrm{C}$. The total difference of the integrated intensity was $17.5 \%$. The RL is low at room temperature due to some of the mobile carriers being trapped. The probability of thermal detrapping increases with increasing temperature. This means that a smaller fraction of the mobile electrons and holes become trapped as the temperatures increases. This results in an increase in the RL signal with increasing temperature (see model in Fig. 5.16(c)). The sample received a total dose of $\sim 1.2 \mathrm{kGy}$ and it is possible that some non-radiative recombination sites are generated by X-ray irradiation that leads to a reduction in the RL. As can be seen in Fig. 6.12(b), the RL systematically decreases with increasing dose for doses above $~ 100$ Gy. 


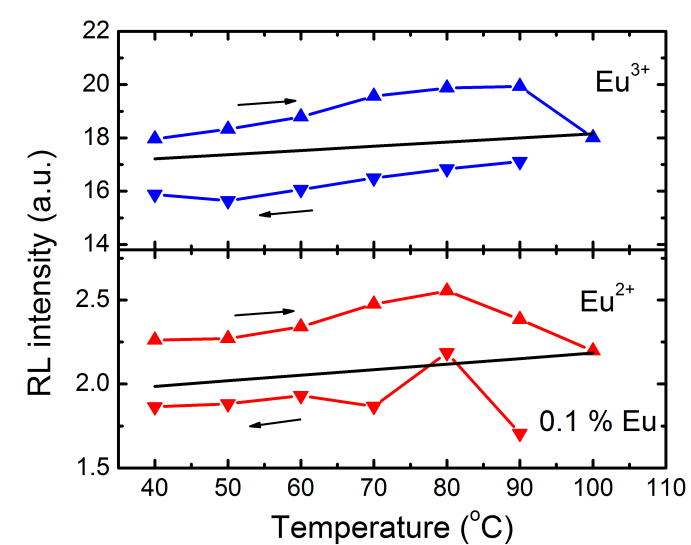

(a)

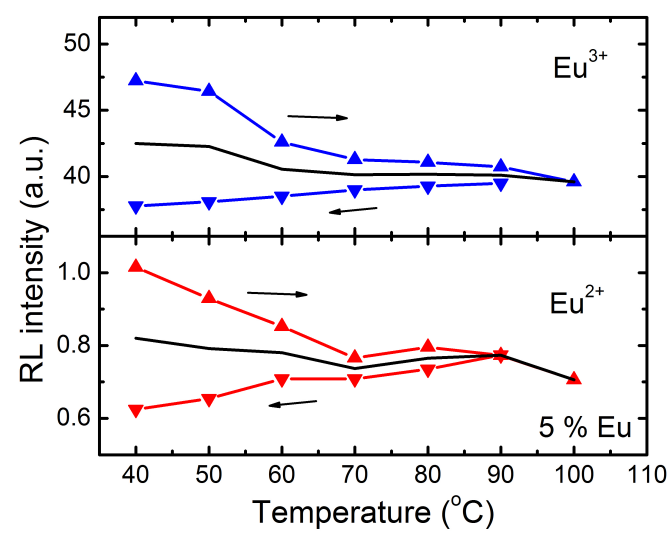

(c)

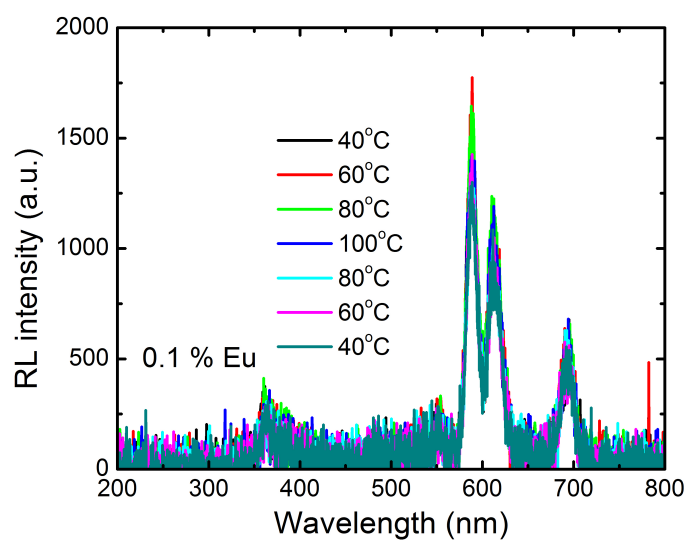

(b)

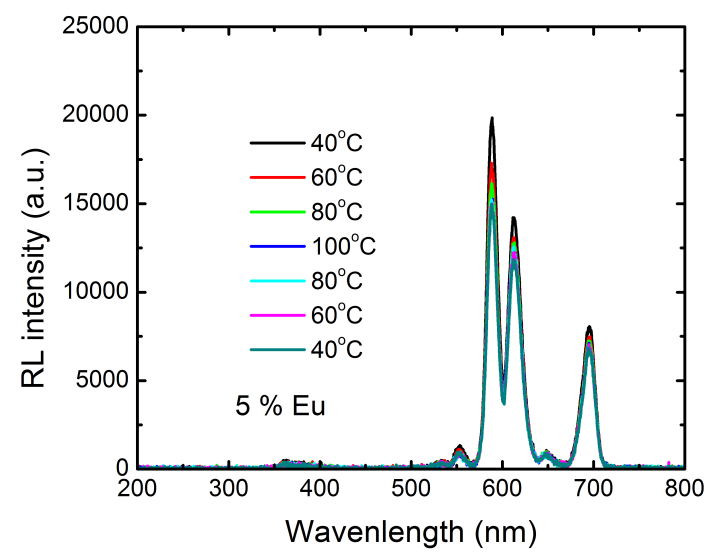

(d)

Figure 6.19: Temperature dependent RL spectra for $\mathrm{NaMgF}_{3}$ nanoparticles doped with 0.1 $\% \mathrm{Eu}(\mathrm{a})$, and $5 \% \mathrm{Eu}(\mathrm{c})$ for different temperatures up to $100{ }^{\circ} \mathrm{C}$ and the respective RL spectra versus the wavelength for different temperatures ((b) for $0.1 \% \mathrm{Eu}$ and (d) for $5 \% \mathrm{Eu})$.

Arrows facing up signal an increase of temperature, and arrows facing down signal a decrease in temperature. $\mathrm{Eu}^{2+}$ is indicated by the red colour and $\mathrm{Eu}^{3+}$ by the blue colour. The lines are guided to the eye.

The $5 \%$ Eu sample shows a decrease in the $\mathrm{Eu}^{2+}$ and $\mathrm{Eu}^{3+} \mathrm{RL}$ compared to room temperature as the temperature is increased to $100{ }^{\circ} \mathrm{C}$. Decreasing the temperature from $100{ }^{\circ} \mathrm{C}$ to $40{ }^{\circ} \mathrm{C}$ results in a further decrease in the $\mathrm{RL}$ where the $\mathrm{RL}$ at $30^{\circ} \mathrm{C}$ is lower than the 
initial value. The initial decrease in the RL with increasing temperature may be due to thermal activation to non-radiative recombination centres as shown in Fig. 6.20. In this model, the RL should then increase as the temperature is reduced from $100{ }^{\circ} \mathrm{C}$ to $30{ }^{\circ} \mathrm{C}$. However, this does not occur. It is possible that some non-radiative recombination sites are generated by X-ray irradiation that leads to a reduction in the RL. This is possible because the total dose during the experiment was $\sim 1.2 \mathrm{kGy}$. As can be seen in Fig. 6.14, the RL systematically decreases with increasing dose for doses above $~ 100$ Gy.

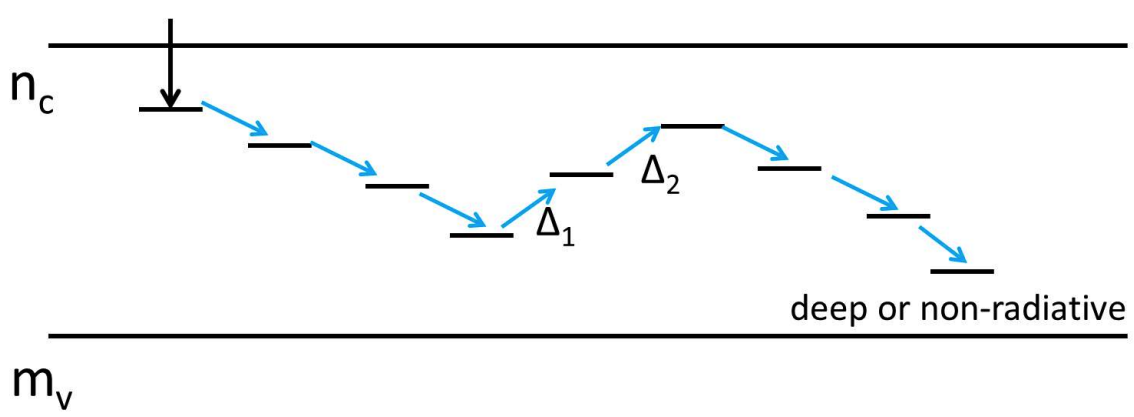

Figure 6.20: Access of non-radiative traps with increasing temperature.

The thermal coefficient of the RL could not be estimated. There was no observable shift in the $\mathrm{RL}$ emission wavelengths of $\mathrm{Eu}^{2+}$ transitions with increasing temperature because the temperature change was too low.

\subsubsection{2 $\mathrm{Mn}$ doped $\mathrm{NaMgF}_{3}$ nanoparticles}

In Fig. 6.21 and 6.22 , the temperature dependent integrated RL spectra of $1 \% \mathrm{Mn}$ and $5 \%$ $\mathrm{Mn}$ doped $\mathrm{NaMgF}_{3}$ and their RL spectra against the wavelength for different temperatures are plotted. $\mathrm{I}_{R L}$ for the $1 \% \mathrm{Mn}$ doped sample decreases by $12.1 \%$ compared to room temperature when increasing temperature up tot $100{ }^{\circ} \mathrm{C}$ and decreases by $1.7 \%$ compared to $100{ }^{\circ} \mathrm{C}$ when decreasing temperature at $40{ }^{\circ} \mathrm{C}$ leading to a total decrease by $13.7 \%$. The 
decrease of the RL intensity with increasing temperature might be due to an energy transfer to a non-radiative recombination site. Therefore, the overall RL intensity decreases (Fig. 6.21(a)). The $1 \% \mathrm{Mn}$ sample shows a decrease in the RL compared to room temperature when the temperature increases up to $100{ }^{\circ} \mathrm{C}$. Decreasing the temperature from $100{ }^{\circ} \mathrm{C}$ to $40{ }^{\circ} \mathrm{C}$ results in a further decrease in the $\mathrm{RL}$ where the $\mathrm{RL}$ intensity when measuring it again at $40{ }^{\circ} \mathrm{C}$ is lower than the initial value. The initial decrease in the RL with increasing temperature may be due to thermal activation to non-radiative recombination centres as shown in Fig. 6.20. In this model, the RL should then increase as the temperature is reduced from $100{ }^{\circ} \mathrm{C}$ to $40^{\circ} \mathrm{C}$. However, this does not occur. The further decrease of the $\mathrm{RL}$ signal with decreasing temperature from $100{ }^{\circ} \mathrm{C}$ to $40{ }^{\circ} \mathrm{C}$ is due to the dose dependence of the RL signal. The sample has received a total dose of $\sim 1.2 \mathrm{kGy}$, and RL measurements showed that the signal will decrease by $2.7 \%$ at this dose (see Fig. 6.16(a)).

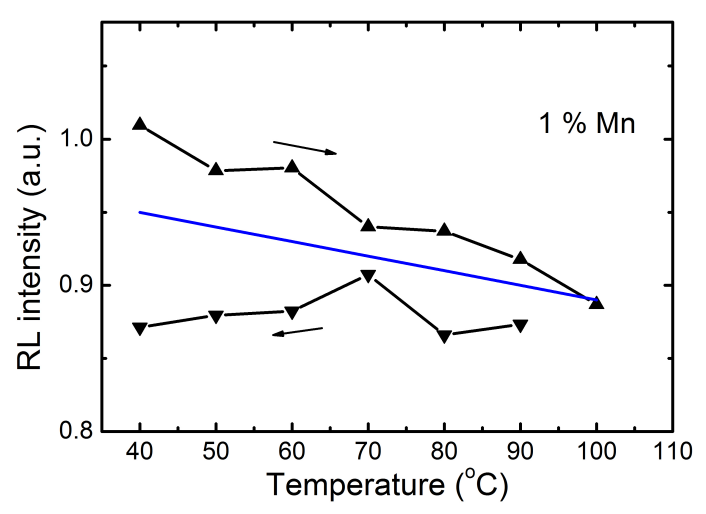

(a)

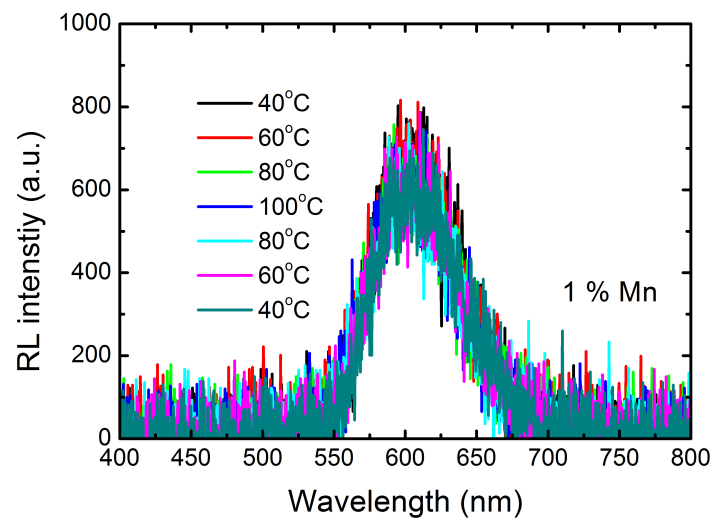

(b)

Figure 6.21: Temperature dependent RL spectra for $\mathrm{NaMgF}_{3}$ nanoparticles doped with $1 \%$ $\mathrm{Mn}$ (a) for different temperatures up to $100{ }^{\circ} \mathrm{C}$ and the respective RL spectra versus the wavelength for different temperatures ((b) for $1 \% \mathrm{Mn})$. Arrows facing up signal an increase of temperature, and arrows facing down signal a decrease in temperature. The lines are guided to the eye.

A different behaviour was observed for the $5 \% \mathrm{Mn}$ doped sample (Fig. 6.22). At first, the 
signal decreases by $3.2 \%$ compared to room temperature as the temperature is increased to $100{ }^{\circ} \mathrm{C}$ and increases by $16.4 \%$ compared to $100{ }^{\circ} \mathrm{C}$ when decreasing temperature at $40{ }^{\circ} \mathrm{C}$ leading to a total increase by $13.7 \%$. At low temperatures, the traps start to get filled. They are not filled unless there is a high enough dose. The appearance of RL does not depend on the filling of the traps. If the temperature increases, the probability of hopping increases. This means that the carriers overcome an energy barrier, $\Delta$, and could recombine in non-radiative recombination centres (Fig. 6.22(c)). For small doses and hence a negligible trapped carrier concentration, the RL signal should decrease with decreasing temperature. This is here not observed. However, it is likely that the dose at each temperature results in a significant carrier trapping. Therefore, as the temperature decreases there may be a competition between reducing the RL via reducing the thermal detrapping probability and increasing the RL due to significant trap filling at each temperature. It is apparent that the second process dominates in Fig. 6.22(a). The same process was also shown in Fig. 5.16(a) for the $\mathrm{NaMgF}_{3}$ polycrystalline materials. The thermal coefficient could not be calculated from the measurements since the difference in the RL intensities is too large for increasing and decreasing temperatures. The temperature was not high enough to observe a shift in the RL emission wavelengths. 


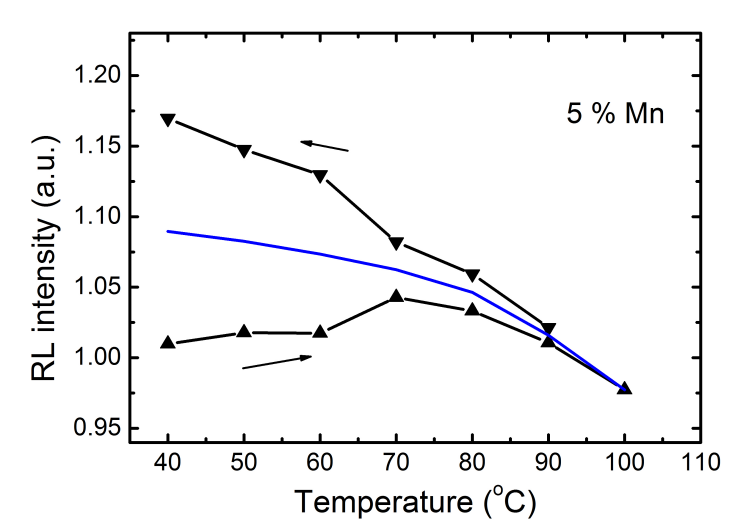

(a)

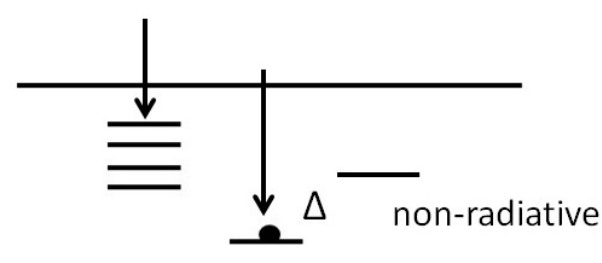

low T

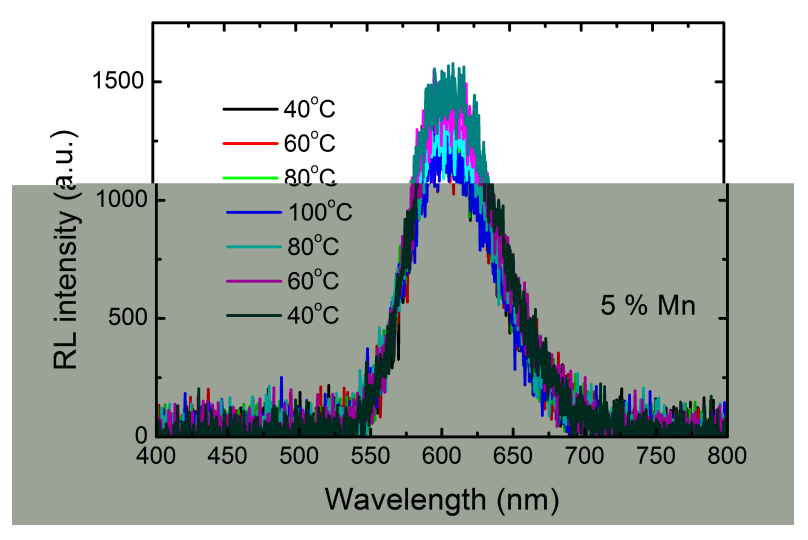

(b)

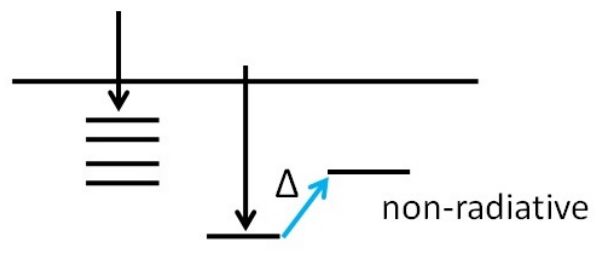

high T

(c)

Figure 6.22: Temperature dependent RL spectra for $\mathrm{NaMgF}_{3}$ nanoparticles doped with5 \% $\mathrm{Mn}$ (a) for different temperatures up to $100{ }^{\circ} \mathrm{C}$ and the respective RL spectra versus the wavelength for different temperatures ((b) for $5 \% \mathrm{Mn}$ ).

Arrows facing up signal an increase of temperature, and arrows facing down signal an decreases in temperature. The lines are guided to the eye.

(c) Non-radiative recombination near a Mn site.

\subsection{Conclusion}

$\mathrm{Eu}^{2+}, \mathrm{Eu}^{3+}$, and a unknown defect centred at around $470 \mathrm{~nm}$ were observed in the PL spectra of the nanoparticles as it previously was observed for the polycrystalline bulk ma- 
terials. Temperature dependent PL measurements for the Eu doped samples show that the unknown defect centred at $470 \mathrm{~nm}$ increases with decreasing temperature. The peak of the unknown defect in the PL excitation spectra is broader at low temperature. The PL intensity of the unknown defect is also dependent on the concentration of the dopant, which means it increases with decreasing Eu concentration. The PL lifetimes of the Eu doped nanoparticles are similar to the bulk materials, when calculated with the $1^{\text {st }}$ and $2^{\text {nd }}$ component of the multi-exponential fit from the PL decay curve. $\tau_{12, \text { ave }}$ of the $\mathrm{Eu}^{3+}$ ion decreases with increasing Eu concentration, which can be attributed to an energy transfer from $\mathrm{Eu}^{3+}$ sites away from the surface to $\mathrm{Eu}^{3+}$ sites near the surface. The quantum efficiency was as high as $99.7 \%$. Temperature dependent PL lifetime measurements show that $\tau_{12, a v e}$ is nearly constant for decreasing temperature. The unknown defect has a long PL lifetime of $49.4 \mathrm{~ms}$ for the $0.1 \%$ sample and the lifetime decreases to $35.5 \mathrm{~ms}$ for the 5 $\% \mathrm{Eu}$ doped sample. It shows thermally activated behaviour with an activation energy of $104 \pm 36 \mathrm{meV}$ for the $1 \%$ doped sample and $95 \pm 40 \mathrm{meV}$ for the $5 \%$ sample. The Judd-Ofelt parameters have been calculated and $\Omega_{2}$ seems to be in the range of other nanoparticle compounds. $\Omega_{4}$ is higher than $\Omega_{2}$ and higher than observed in other compounds as $\mathrm{LaF}_{3}$. The $\mathrm{NaMgF}_{3}$ :Eu nanoparticles showed similar activation energies of the TL glow curves peaks as it was researched for the Eu doped bulk materials. This means that the peaks might arise from the same defects. $\mathrm{Eu}^{2+}$ and $\mathrm{Eu}^{3+}$ were measured in the RL spectra. The unknown defect centred at $\sim 470 \mathrm{~nm}$ was not observed in the RL spectra of the Eu doped samples. $\mathrm{I}_{R L}$ is independent of dose from $10 \mathrm{mGy}$ to $\sim 500 \mathrm{~Gy}$. For doses higher than $500 \mathrm{~Gy}$, the RL intensity decreases. The integrated RL intensity of the $\mathrm{Eu}^{3+}$ for the 0.5 $\%$ sample decreases by $2.6 \%$ at $12.7 \mathrm{kGy}$. The $5 \%$ doped nanoparticles show the highest decrease by $13.6 \%$ at $12.7 \mathrm{kGy}$ for the $\mathrm{Eu}^{3+}$ ion. The RL intensity of the $\mathrm{Eu}^{2+}$ ion decreases at a faster rate for all of the samples. In general, the RL intensity is less dependent on the dose as it was observed for the bulk samples, which means there are different defect distributions in the nanoparticles. The RL intensity shows a higher dependence on the temperature than the bulk materials and the difference in the RL signal for increasing and decreasing temperature is too large to calculate the thermal coefficient of the RL intensity. 
PL measurements show the regular $\mathrm{Mn}^{2+}$ site centred at $600 \mathrm{~nm}$ and also an additional highly distorted site centred at $500 \mathrm{~nm}$, which also was observed for the $\mathrm{NaMgF}_{3}$ bulk materials. The PL decay curve of the $\mathrm{Mn}^{2+}$ nanoparticles could be fitted two-exponential. The regular site shows a higher lifetime than the distorted site, which might suggest that the distorted site is near a non-radiative centre. TL measurements for the Mn doped nanoparticles show more low temperature peaks and different activation energies for the peaks compared to the Mn doped bulk materials. This means that there is a different defect distribution compared to the bulk materials. $\mathrm{Mn}^{2+}$ was observed in the RL spectra and the integrated intensity slightly decreases above $\sim 1 \mathrm{kGy}$ for $5 \% \mathrm{Mn}^{2+}$. For the $1 \%$ and 2 \% Mn doped samples, the integrated RL intensity decreases at a faster rate. The RL of the Mn doped nanoparticles is dependent on the temperature, but the thermal coefficient of the RL could not be calculated. As previously mentioned in chapter 5 for the polycrystalline $\mathrm{NaMgF}_{3}$ materials, optical bleaching of the nanoparticles after the temperature change should be applied. 


\section{Bulk polycrystalline $\mathrm{RbMgF}_{3}$}

In this chapter, the optical, radioluminescent and thermoluminescent properties of Eu and Mn doped $\mathrm{RbMgF}_{3}$ will be discussed. The samples were prepared as described in the experimental description given in chapter 4.1.1. $10 \mathrm{~g}$ of each sample was produced using high purity Rubidium fluoride $(\mathrm{RbF})$, Magnesium fluoride $\left(\mathrm{MgF}_{2}\right)$, Europium(II) fluoride $\left(\mathrm{EuF}_{2}\right)$ or Manganese(II) fluoride $\left(\mathrm{MnF}_{2}\right) \cdot \mathrm{RbMgF}_{3}$ has a hexagonal crystal structure with the space group $P_{6_{3} / m m c}$ and a melting point of $\sim 912{ }^{\circ} \mathrm{C}$. The bandgap is about $7.32 \mathrm{eV}$ [79], and the crystal field splitting between the $4 \mathrm{f}-5 \mathrm{~d}$ level is around $970 \mathrm{~cm}^{-1}(0.12 \mathrm{eV})$ [59]. The samples which will be discussed were doped with $0.2 \% \mathrm{Eu}$ or $0.2 \% \mathrm{Mn}$ and their appearance was transparent.

\subsection{Photoluminescence and PL lifetime measurements}

PL measurements were performed with the Jobin-Yvon FluoroLog ${ }^{\circledR}$.

\subsubsection{Eu doped polycrystalline $\mathrm{RbMgF}_{3}$}

The PL spectrum of $\mathrm{RbMgF}_{3}: 0.2 \% \mathrm{Eu}$ is shown in Fig. 7.1, which arises from the $\mathrm{Eu}^{2+}$ transitions. The sharp peak in the PL emission is due to the transition from the ${ }^{6} \mathrm{P}_{7 / 2}$ and ${ }^{6} \mathrm{P}_{5 / 2}$ excited states to the ${ }^{8} \mathrm{~S}_{7 / 2}$ ground state with an emission at $360 \mathrm{~nm}$, and the broad peak is from the band emission from the $4 \mathrm{f}^{6} 5 \mathrm{~d}\left(\mathrm{E}_{g}\right)$ excited states to the $4 \mathrm{f}^{7}$ ground state. 
$\mathrm{RbMgF}_{3}$ does not show a broad peak of the $5 \mathrm{~d}$ level in the linear PL spectra as it was observed for the $\mathrm{NaMgF}_{3}$ (Fig. 5.1). This is due to the energy difference between the $4 \mathrm{f}$ and $5 \mathrm{~d}$ levels. The energy difference is $\sim 9700 \mathrm{~cm}^{-1}(1.2 \mathrm{eV})$ for $\mathrm{NaMgF}_{3}$, and $\sim 970 \mathrm{~cm}^{-1}$ $(0.12 \mathrm{eV})$ for $\mathrm{RbMgF}_{3}$ [9, 59, 79, 80, 81]. Although the $5 \mathrm{~d}$ transition could be observed in the PL spectra when plotting it logarithmic (Fig. 7.1(b)) [4]. The small energy difference causes the $5 \mathrm{~d}$ to be quenched by the $4 \mathrm{f}$ (Fig. 7.1(a)). The sharp peak at $360 \mathrm{~nm}$ from the 4f level is also detected at $360 \mathrm{~nm}$ in the $\mathrm{NaMgF}_{3}$ bulk materials (Fig. 5.1). The reason there is no shift is that $4 \mathrm{f}$ transitions are shielded by the electrons in $5 \mathrm{~d}$ and therefore, not strongly affected by the crystal field.

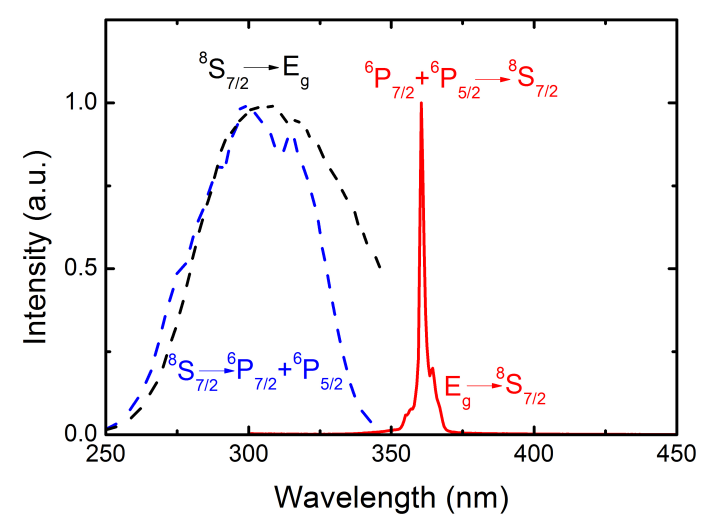

(a)

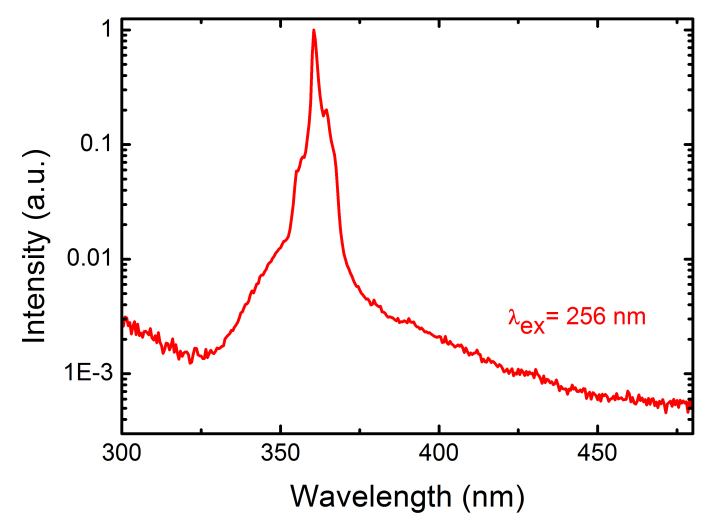

(b)

Figure 7.1: (a) PL excitation (dashed curve, $\lambda_{e m}=360$ (blue) $\mathrm{nm}$ and $420 \mathrm{~nm}$ (black)) and emission (solid curve, $\lambda_{e x}=256 \mathrm{~nm}$ ) spectra for $\mathrm{RbMgF}_{3} 0.2 \% \mathrm{Eu}$.

(b) Logarithmic plot of the PL emission spectra at $\lambda_{e x}=256 \mathrm{~nm}$.

PL measurements were done before and after heavy irradiation. In order to perform this, the sample was placed on the holder for the PL measurements. The holder was transported in a light-tight box to the X-ray generator. The sample was irradiated up to a dose of $\sim 12.7$ kGy with the X-ray generator operating at $40 \mathrm{~mA}$ and $40 \mathrm{kV}$. After radiation, the holder with sample was transported back in the light-tight box to the spectrometer and the PL measurement was repeated with the sample in the same position. 


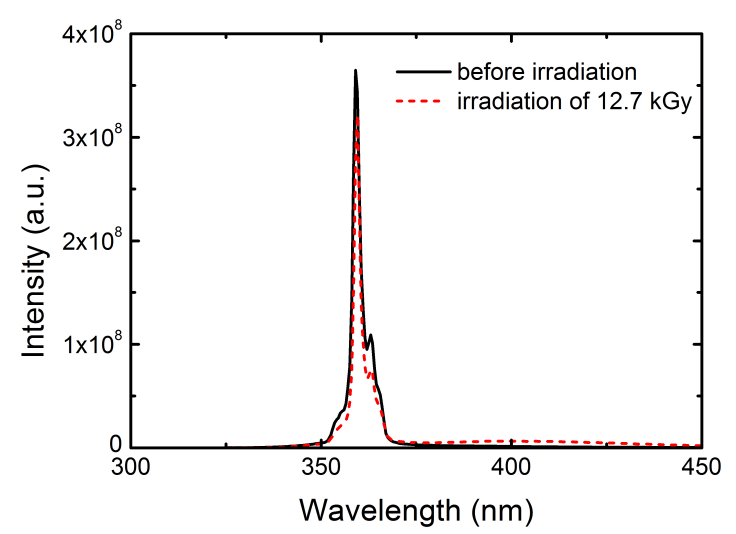

(a)

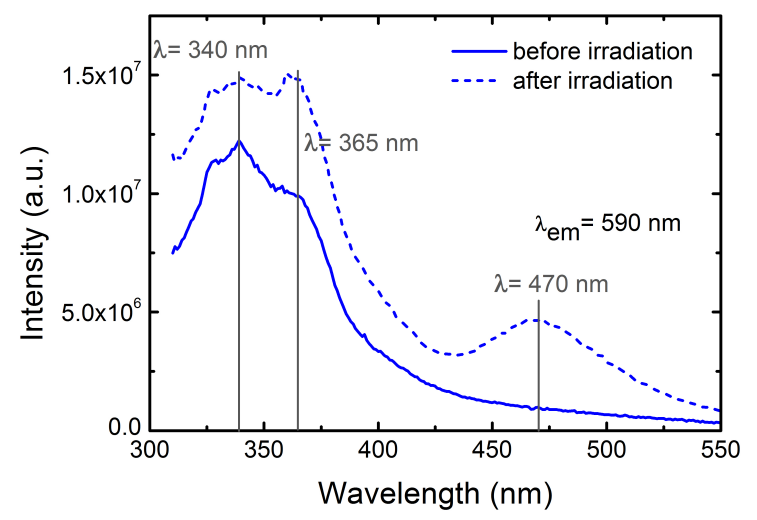

(c)

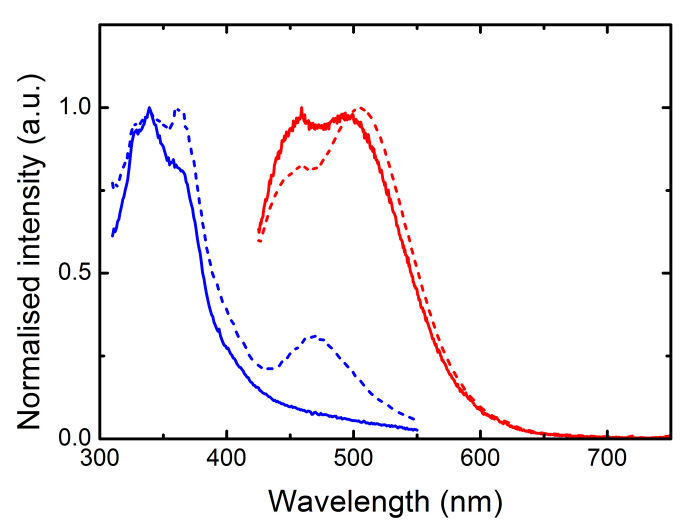

(b)

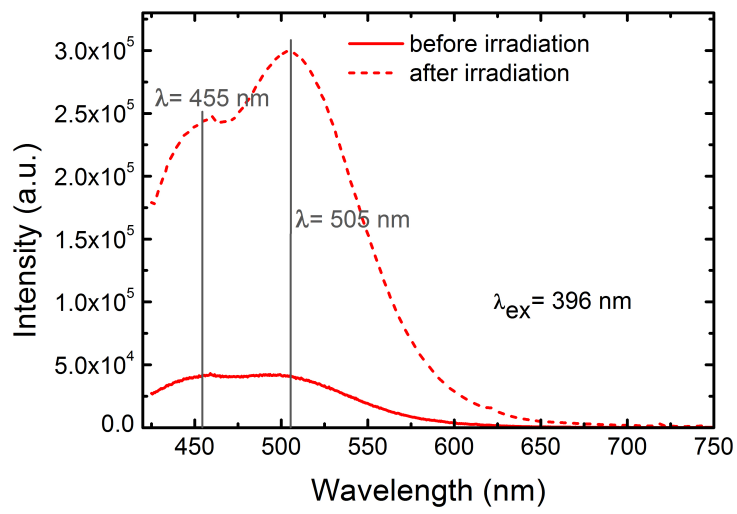

(d)

Figure 7.2: (a) Absolute PL intensity of the emission spectra, $\lambda_{e x}=256 \mathrm{~nm}$, for $\mathrm{RbMgF}_{3}$ $0.2 \%$ Eu before (solid curve) and after irradiation with a dose of $12.7 \mathrm{kGy}$ (dashed curve).

(b) PL excitation at $\lambda_{e m}=590 \mathrm{~nm}$ (blue: solid curve - before irradiation, dashed curve - after irradiation) and emission at $\lambda_{e x}=396 \mathrm{~nm}$ (red: solid curve - before irradiation, dashed curve - after irradiation with a dose of $12.7 \mathrm{kGy}$ ) spectra for the $\mathrm{Eu}^{3+}$ ion.

(c) Absolute PL intensity of the excitation spectra, $\lambda_{e m}=590 \mathrm{~nm}$, before (solid curve) and after irradiation with a dose of $12.7 \mathrm{kGy}$ (dashed curve).

(d) Absolute PL intensity of the emission spectra, $\lambda_{e x}=396 \mathrm{~nm}$, before (solid curve) and after irradiation with a dose of $12.7 \mathrm{kGy}$ (dashed curve). 
Fig. 7.2(a) shows the absolute PL intensity of the emission spectra for $\mathrm{Eu}^{2+}$ before (solid curve) and after (dashed curve) heavy irradiation. The PL intensity decreases by $24 \%$ after receiving a dose of $\sim 12.7 \mathrm{kGy}$, but the shape of the $\mathrm{Eu}^{2+}$ transition remains unchanged. When exciting the sample at $396 \mathrm{~nm} \mathrm{no} \mathrm{Eu}^{3+}$ emission is observed (Fig. 7.2(b)). In Fig. 7.2 (c), the excitation spectra for an emission at $590 \mathrm{~nm}$ is plotted. The excitation spectrum before irradiation consists out of two peaks, one centred at $340 \mathrm{~nm}$ and one at $365 \mathrm{~nm}$ and these ones are indicated in Fig. 7.2(c). After heavy irradiation with a dose of $\sim 12.7 \mathrm{kGy}$, the peaks at $340 \mathrm{~nm}$ and $365 \mathrm{~nm}$ increase and an additional peak at 470 $\mathrm{nm}$ appears. These additional peak was also observed for $\mathrm{NaMgF}_{3}$ bulk samples (see Fig. $5.3(\mathrm{c}))$. The emission spectra, which was excited at $\lambda_{e x}=396 \mathrm{~nm}$, peak around $505 \mathrm{~nm}$ before irradiation (Fig. 5.3(d)), while after irradiation this broad band increases and a new peak with a maximum at $455 \mathrm{~nm}$ appears. This new band at $455 \mathrm{~nm}$ can be assigned to some defects. The precise origin of these defects is unknown, but can probably be ascribed to the same defect previously observed in $\mathrm{NaMgF}_{3}: \mathrm{Eu}$ bulk materials (see Fig. 5.3 $(\mathrm{c}+\mathrm{d})$ ). For the $\mathrm{NaMgF}_{3}$ sample doped with Eu, the unknown defects were observed at $340 \mathrm{~nm}$ and $470 \mathrm{~nm}$ in the PL excitation spectra. There was no extra peak detected at $365 \mathrm{~nm}$ (Fig. 5.3 (c)). The peaks were detected at $\sim 450 \mathrm{~nm}$ and $\sim 520 \mathrm{~nm}$ for the $\mathrm{NaMgF}_{3}$ polycrystalline samples (Fig. 5.3(d)). Therefore, it can be assumed that these unknown defects arise from the same defects.

PL lifetime measurements were performed with the Jobin-Yvon FluoroLog ${ }^{\circledR}$ for the $\mathrm{Eu}^{3+}$ and with the Cary50 for the $\mathrm{Eu}^{2+}$ sites. The PL lifetimes for the $0.2 \% \mathrm{Eu}^{2+}$ doped sample were calculated by fitting the curve with a two-exponential decay with the method explained in section 3.2.4. For an emission wavelength of $360 \mathrm{~nm}$ the calculated lifetime was $1.95 \pm 0.20 \mathrm{~ms}$, while for a wavelength of $400 \mathrm{~nm}$ a lifetime of $0.51 \pm 0.05 \mathrm{~ms}$ was obtained $\left(\lambda_{e x}=256 \mathrm{~nm}\right)$, see Fig. $7.3(\mathrm{a}+\mathrm{b})$. The lifetime of the $360 \mathrm{~nm}$ emission in the $\mathrm{RbMgF}_{3}$ sample is slightly higher than the lifetime for $\mathrm{NaMgF}_{3}$ samples which was calculated with $0.63 \pm 0.06 \mathrm{~ms}$, which can result from a different symmetry of the crystal field. For the unknown defect an average PL lifetime of $48.5 \pm 4.9 \mathrm{~ms}$ (at $\lambda_{e m}=460 \mathrm{~nm}$ and $\lambda_{e x}=375$ 
nm) was measured. $\mathrm{NaMgF}_{3}$ : Eu had a PL lifetime of $68 \pm 7 \mathrm{~ms}$ for the unknown peak.

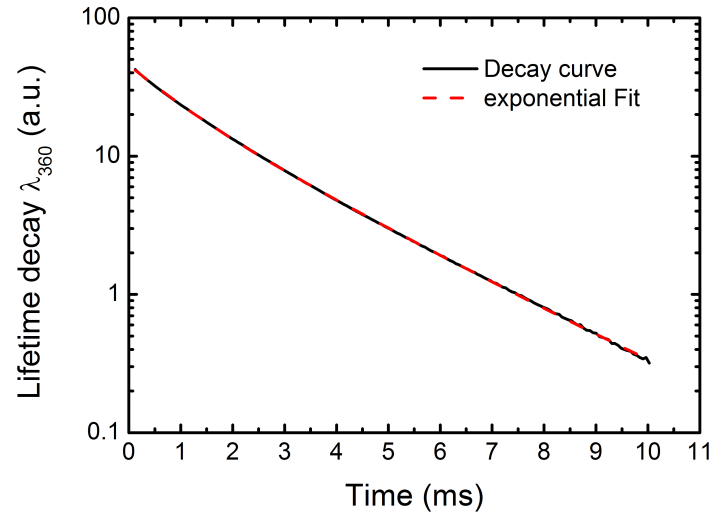

(a)

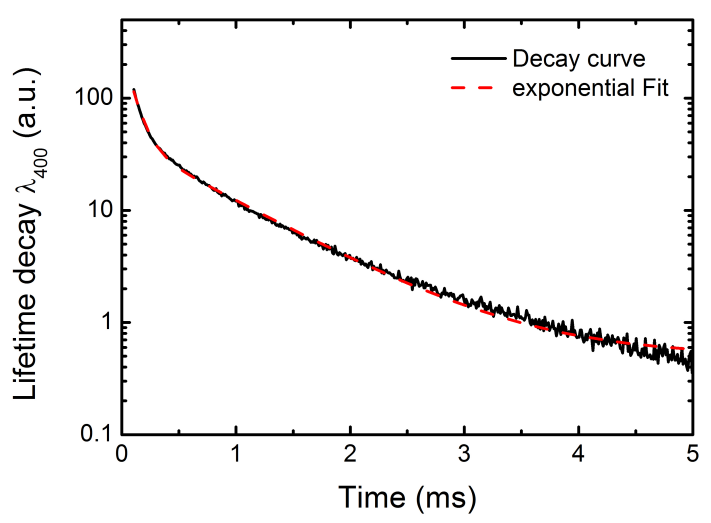

(b)

Figure 7.3: (a) PL lifetime decay of $0.2 \% \mathrm{Eu}$ doped $\mathrm{RbMgF}_{3}$ at $\lambda_{\text {em }}=360 \mathrm{~nm}$ (solid curve) and (b) $\lambda_{e m}=400 \mathrm{~nm}$ emission (solid curve). The dashed curve is the exponential fit. Both lifetimes were measured at $\lambda_{e x}=256 \mathrm{~nm}$.

The different compounds of the average PL lifetimes are listed in Tab. 7.1. For the $\mathrm{Eu}^{2+}$ emission at $360 \mathrm{~nm}$, the existence of two separate sites with different lifetimes of $0.90 \pm 0.09$ ms and $2.22 \pm 0.22 \mathrm{~ms}$ was observed. The $\mathrm{Eu}^{2+}$ emission at $400 \mathrm{~nm}$ also showed two sites with lifetimes of $0.09 \pm 0.01 \mathrm{~ms}$ and $0.79 \pm 0.08 \mathrm{~ms}$. For the unknown peak, two different lifetimes of $8.74 \pm 0.88 \mathrm{~ms}$ and $106 \pm 11 \mathrm{~ms}$ were observed. The longer the lifetime, the less distorted the site is.

Table 7.1: Different compounds with the pre factors of the PL lifetimes of Eu doped $\mathrm{RbMgF}_{3}$

\begin{tabular}{|l|l|l|l|l|}
\hline$\lambda_{e m}(\mathrm{~nm})$ & factor $\mathrm{A}_{1}$ & $\tau_{1}(\mathrm{~ms})$ & factor $\mathrm{A}_{2}$ & $\tau_{2}(\mathrm{~ms})$ \\
\hline \hline 360 & 18 & $0.90 \pm 0.09$ & 28 & $2.22 \pm 0.22$ \\
\hline 400 & 240 & $0.09 \pm 0.01$ & 42 & $0.79 \pm 0.08$ \\
\hline 500 & 703 & $8.74 \pm 0.87$ & 88 & $106 \pm 11$ \\
\hline
\end{tabular}




\subsubsection{Mn doped polycrystalline $\mathrm{RbMgF}_{3}$}

The PL emission and excitation spectra for the $\mathrm{RbMgF}_{3}$ samples doped with $0.2 \% \mathrm{Mn}$ are plotted in Fig. 7.4 (a+b). The PL excitation and emission peaks are consistent with $\mathrm{Mn}^{2+}$. $\mathrm{Mn}^{2+}$ substitutes for $\mathrm{Mg}$, and $\mathrm{RbMgF}_{3}$ has the $\mathrm{P}_{6_{3} / m m c}$ space group with two inequivalent $\mathrm{Mg}$ sites. Therefore, two $\mathrm{Mn}^{2+}$ sites where expected: one site, $\mathrm{Mn} 1$, has $\mathrm{C}_{3 v}$ symmetry and the other site, $\mathrm{Mn} 2$, has $\mathrm{D}_{3 d}$ symmetry [3]. The difference in the crystal field between the two sites in the compound is large enough to be detected in the PL excitation spectra resulting in a shift in the PL excitation peaks, when detecting the emission at $550 \mathrm{~nm}$ or $590 \mathrm{~nm}$ [96, 97]. The PL emission, when excited at $396 \mathrm{~nm}$, exists of one peak at $580 \mathrm{~nm}$. For the $\mathrm{NaMgF}_{3}$ : Mn bulk materials, the transition from the ${ }^{6} \mathrm{~A}_{1 g}$ was found at $600 \mathrm{~nm}$. There are no additional peaks observed from a highly distorted Mn site as it was detected in $\mathrm{NaMgF}_{3}$ samples at $500 \mathrm{~nm}$ (Fig. 7.4(b)).

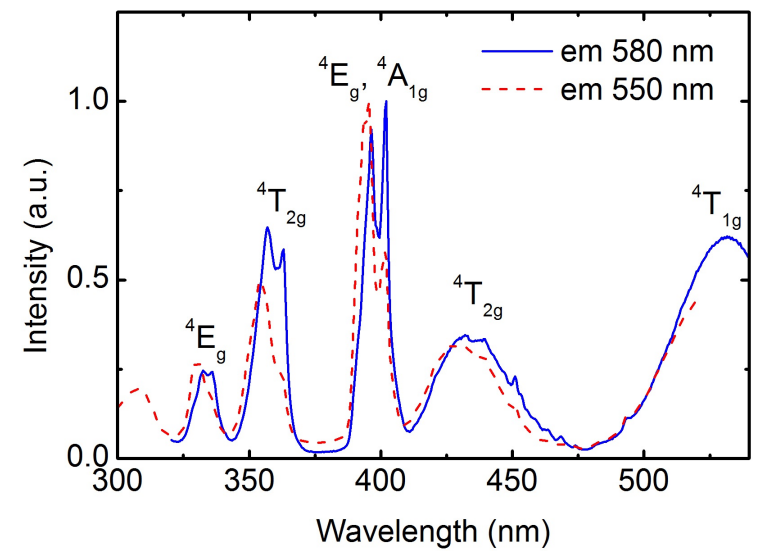

(a)

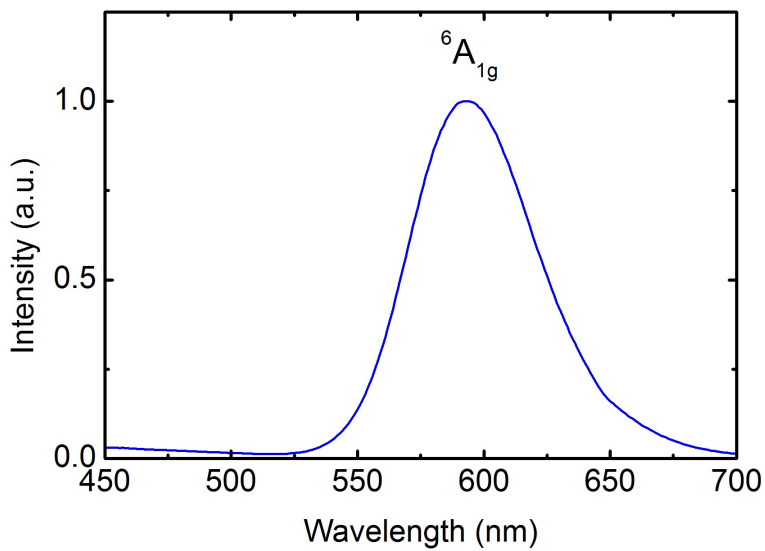

(b)

Figure 7.4: (a) PL excitation spectrum at $\lambda_{e m}=550 \mathrm{~nm}$ (dashed curve) and $\lambda_{e m}=580 \mathrm{~nm}$ emission (solid curve).

(b) PL emission spectrum for $\mathrm{RbMgF}_{3}$ doped with $0.2 \% \mathrm{Mn}$ excited at 396 $\mathrm{nm}$. 


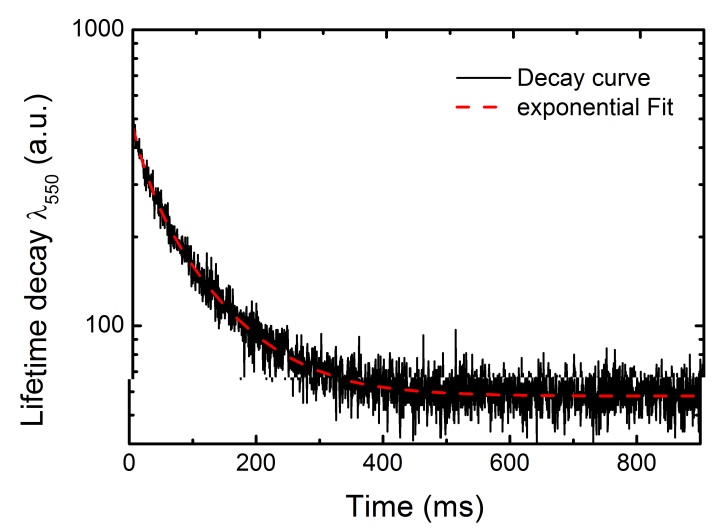

(a)

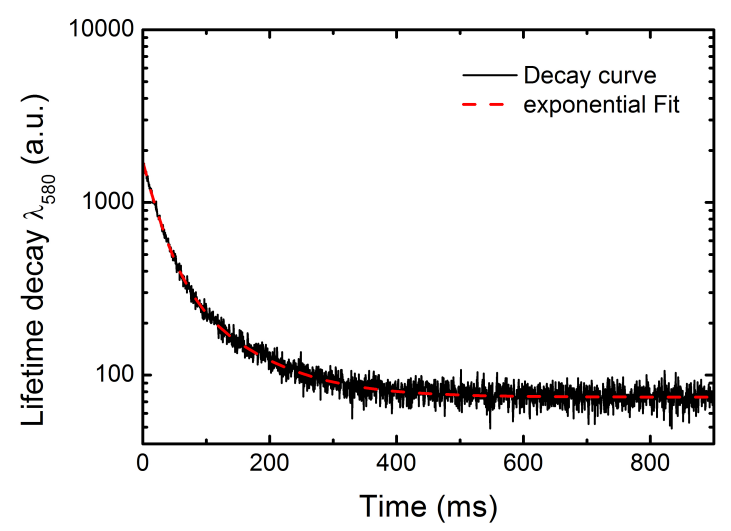

(b)

Figure 7.5: (a) PL lifetime decay of $0.2 \% \mathrm{Mn}$ doped $\mathrm{RbMgF}_{3}$ at $\lambda_{\text {em }}=550 \mathrm{~nm}$ (solid curve) and (b) $\lambda_{e m}=580 \mathrm{~nm}$ emission (solid curve). The dashed curve is the exponential fit. Both lifetimes were measured at $\lambda_{e x}=465 \mathrm{~nm}$.

The average PL lifetime measurements were performed with the Jobin-Yvon FluoroLog ${ }^{\circledR}$ for the $\mathrm{Mn}^{2+}$ sites. For the $0.2 \% \mathrm{Mn}$ doped sample, an average lifetime of $87.7 \pm 8.8 \mathrm{~ms}$ for the $550 \mathrm{~nm}$ emission and $62.9 \pm 6.3 \mathrm{~ms}$ for the $580 \mathrm{~nm}$ emission was measured (at $\lambda_{e x}=$ $465 \mathrm{~nm}$ ), using a two-exponential fit for the PL lifetime decay as explained in section 3.2.4. The different compounds of the average PL lifetimes are listed in Tab. 7.2. For the $\mathrm{Mn}^{2+}$ emission at $550 \mathrm{~nm}$, the existence of two separate sites with different lifetimes of $21.8 \pm 2.2$ ms and $96 \pm 10 \mathrm{~ms}$ were observed. The $\mathrm{Mn}^{2+}$ emission centred at $580 \mathrm{~nm}$ also showed two sites with lifetimes of $25.0 \pm 2.5 \mathrm{~ms}$ and $97 \pm 10 \mathrm{~ms}$. The different symmetry of the two sites of $\mathrm{Mn}^{2+}$ could cause the different lifetimes of the $550 \mathrm{~nm}$ and $580 \mathrm{~nm}$ emission. The PL lifetime for the $\mathrm{NaMgF}_{3} \mathrm{Mn}$ doped samples was $98 \pm 10 \mathrm{~ms}$ for $0.5 \% \mathrm{Mn}$, and $91.4 \pm 9.1 \mathrm{~ms}$ for $1 \% \mathrm{Mn}$ for the regular $\mathrm{Mn}^{2+}$ site. 
Table 7.2: Different compounds with the pre factors of the PL lifetimes of Mn doped $\mathrm{RbMgF}_{3}$.

\begin{tabular}{|l|l|l|l|l|}
\hline$\lambda_{e m}(\mathrm{~nm})$ & factor $\mathrm{A}_{1}$ & $\tau_{1}(\mathrm{~ms})$ & factor $\mathrm{A}_{2}$ & $\tau_{2}(\mathrm{~ms})$ \\
\hline \hline 550 & 145 & $21.8 \pm 2.2$ & 267 & $96 \pm 10$ \\
\hline 580 & 1275 & $25.0 \pm 2.5$ & 366 & $97 \pm 10$ \\
\hline
\end{tabular}

\subsection{Thermoluminescence measurements}

The TL measurements were performed using the same method as previously described in section 5.2 for $\mathrm{NaMgF}_{3}$. The glow curves were obtained after irradiating the sample for 5 min and using a heating rate of $1 \mathrm{~K} / \mathrm{s}$.

\subsubsection{Eu doped polycrystalline $\mathrm{RbMgF}_{3}$}

The TL glow curve is plotted in Fig. 7.6(a) and activation energies of the peaks of the glow curves are summarised in Tab. 7.3. The glow curve peaks were obtained using first order kinetics glow curve deconvolution software and by minimizing the number of peaks during fitting of the glow curve (see also Fig. 7.6(a) - dashed curves). Fig. 7.6(b) shows the TL intensity plotted against the wavelength for different temperatures. There was no TL from $\mathrm{Eu}^{3+}$ detected but the TL spectra consists of a broad peak centred around $\sim 560 \mathrm{~nm}$ as well as the peak at $360 \mathrm{~nm}\left(\right.$ Fig. $7.6(\mathrm{~b}+\mathrm{c})$ ). This band at $560 \mathrm{~nm}$ could not arise from $\mathrm{Mn}^{2+}$, because there was no evidence of $\mathrm{Mn}^{2+}$ in the PL spectra (Fig. 7.1(a)). The deconvolution of the glow curve was performed for the TL emission at $360 \mathrm{~nm}$ (Fig. 7.6(a)) and for the peak around $560 \mathrm{~nm}$ (Fig. $7.6(\mathrm{c})$ ). The $\mathrm{Eu}^{2+}$ ion in the polycrystalline $\mathrm{RbMgF}_{3}$ bulk sample has two low temperature peaks at $\sim 373 \mathrm{~K}$ and $\sim 408 \mathrm{~K}$ with activation energies of $0.53 \mathrm{eV}$ and $1.07 \mathrm{eV}$, respectively. The main three peaks are found at $482 \mathrm{~K}, 552 \mathrm{~K}$ and $555 \mathrm{~K}$, with respectively an activation energy of $0.92 \mathrm{eV}, 0.5 \mathrm{eV}$ and $1.7 \mathrm{eV}$. Neither 
$\mathrm{NaMgF}_{3}$ or $\mathrm{RbMgF}_{3}$ bulk materials had similar TL glow curve peaks (Tab. 5.8). The $\mathrm{NaMgF}_{3}$ nanoparticles showed a TL peak at $\sim 551 \mathrm{~K}$ with an activation energy of $0.5 \mathrm{eV}$ (Tab. 6.10), which was also observed in the $\mathrm{RbMgF}_{3}$ bulk materials. The $5 \mathrm{~d}$ emission seems to increase with increasing temperature as shown in Fig. 7.6(a) and this might be due to the same effect as for the $\mathrm{NaMgF}_{3}$ samples where it has been explained previously. The probability for the transition between the $4 \mathrm{f}$ and $5 \mathrm{~d}$ level, $\tau_{f \rightarrow d}$, is dependent on the energy difference between the $4 \mathrm{f}$ and $5 \mathrm{~d}$ level, $\Delta$, and the temperature, T. This can be estimated from the following equation,

$$
\tau_{f \rightarrow d}=\tau_{o} e^{-\frac{\Delta}{k T}}
$$

where $\mathrm{k}$ is the Boltzmann's constant (Fig. 5.8(c)). This means that with increasing temperature the energy difference between the $4 \mathrm{f}$ and $5 \mathrm{~d}$ level is decreasing and this will result in a higher probability for the transition from the $4 \mathrm{f}$ to the $5 \mathrm{~d}$ levels. Therefore at higher temperatures more of the $4 \mathrm{f}$ levels emission will be quenched by the $5 \mathrm{~d}$ level resulting in an increase of the emission from the $5 \mathrm{~d}$ level (Fig. 7.6(b)).

The TL glow curve of the unknown defect with a peak at $\sim 560 \mathrm{~nm}$ is plotted in Fig. 7.6(c). It has a similar behaviour as the TL emission of the $\mathrm{Eu}^{2+}$ ion (Fig. 7.6(a)). In Tab. 7.3, the temperatures and activation energies of the TL glow curve peaks are listed and it can be observed that these are similar for the unknown defect and for the $\mathrm{Eu}^{2+}$ ion. The TL peak intensities of the unknown defect and $\mathrm{Eu}^{2+}$ are similar as well. The highest TL intensity arises from the peak with a maximum at $~ 550 \mathrm{~K}$. In the PL spectra a broad peak was observed at $470 \mathrm{~nm}$ (Fig. $7.2(\mathrm{~d})$ ) and it is likely that the unknown peak in the TL spectra arises from the same defect. No TL emission from the broad peak at $560 \mathrm{~nm}$ was observed in the $\mathrm{NaMgF}_{3}$ bulk materials or nanoparticles. Potential additional peaks above $620 \mathrm{~K}$ could not be detected due to the temperature limitation of the equipment used. 


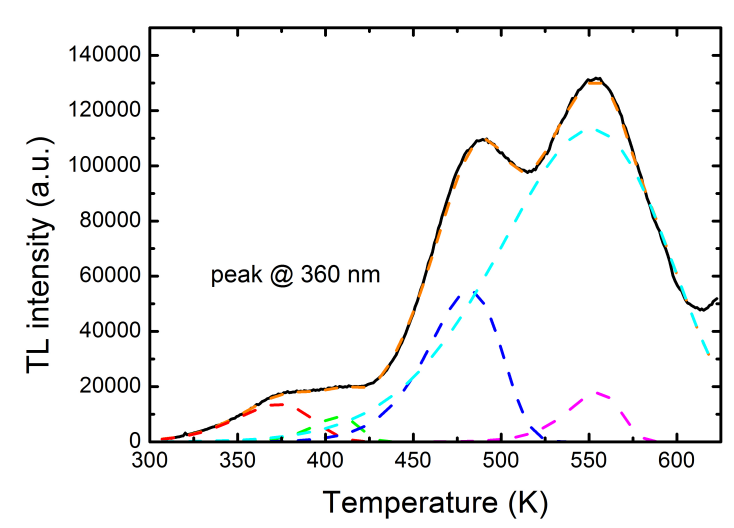

(a)

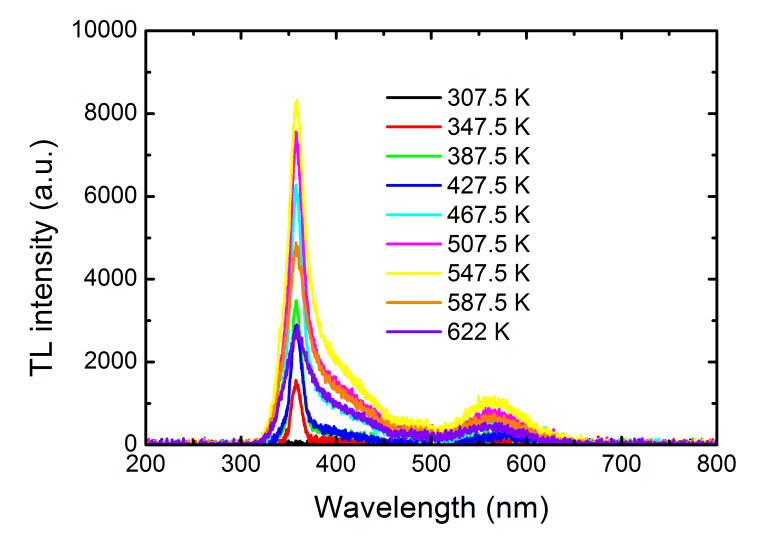

(b)

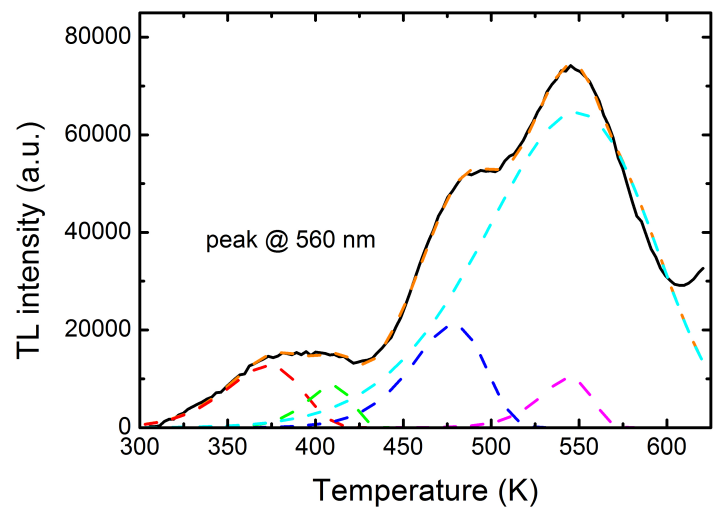

(c)

Figure 7.6: (a) TL for the $\mathrm{RbMgF}_{3}$ sample doped with $0.2 \% \mathrm{Eu}$ (solid curve). The TL data was detected with the PMT after 5 min X-ray irradiation $(40 \mathrm{kV}, 40 \mathrm{~mA})$ at a heating rate of $1 \mathrm{~K} / \mathrm{s}$. The fitted peaks were obtained using first order kinetics glow curve deconvolution (dashed curves).

(b) TL spectra vs. wavelength for the different temperatures detected with the CCD after 5 min X-ray irradiation.

(c) TL for the unknown peak between $500 \mathrm{~nm}$ and $650 \mathrm{~nm}$ (solid curve). TL was detected with the CCD after 5 min of X-ray irradiation and the peaks were fitted using first order kinetics glow curve deconvolution (dashed curves). 
Table 7.3: The activation energies of the individual glow curve peaks for the $\mathrm{RbMgF}_{3}: 0.2$ $\%$ Eu sample obtained by first order kinetics glow curve deconvolution. The top line is the activation energy of the peaks for the for the glow curve.

\begin{tabular}{|l|l|l|l|l|l|}
\hline & $\begin{array}{l}0.53 \pm 0.05 \\
\mathrm{eV}\end{array}$ & $\begin{array}{l}1.07 \pm 0.11 \\
\mathrm{eV}\end{array}$ & $\begin{array}{l}0.92 \pm 0.09 \\
\mathrm{eV}\end{array}$ & $\begin{array}{l}0.50 \pm 0.05 \\
\mathrm{eV}\end{array}$ & $\begin{array}{l}1.70 \pm 0.17 \\
\mathrm{eV}\end{array}$ \\
\hline \hline $\begin{array}{l}0.2 \% \mathrm{Eu} \\
\text { peak height }\end{array}$ & $373 \mathrm{~K}$ & $408 \mathrm{~K}$ & $482 \mathrm{~K}$ & $552 \mathrm{~K}$ & $555 \mathrm{~K}$ \\
13500 & 8900 & 54600 & 113100 & 17600 \\
\hline unknown peak & $373 \mathrm{~K}$ & $410 \mathrm{~K}$ & $478 \mathrm{~K}$ & $549 \mathrm{~K}$ & $544 \mathrm{~K}$ \\
peak height & 12600 & 8500 & 20900 & 64500 & 10100 \\
\hline
\end{tabular}

\subsubsection{Mn doped polycrystalline $\mathrm{RbMgF}_{3}$}

TL spectra from a polycrystalline $\mathrm{RbMgF}_{3}$ sample doped with $\mathrm{Mn}^{2+}$ are plotted in Fig. 7.7 and the activation energies of the fitted peaks are summarised in Tab. 7.4. An irradiation time of 1 minute was used when the data was collected using a PMT (Fig. 7.7(a))and 5 min for the CCD (Fig. 7.7(b)). The TL emission mainly arises from the $\mathrm{Mn}^{2+}$ ions with an emission band around $580 \mathrm{~nm}$. The TL glow curves were fitted using first order kinetics glow curve deconvolution and by trying to minimise the number of peaks. The glow curve consists out of two main peaks located at $346 \mathrm{~K}$ and $555 \mathrm{~K}$. There are also two weaker peaks detected at $375 \mathrm{~K}$ and $595 \mathrm{~K}$. The activation energy of the $\sim 346 \mathrm{~K}$ peak is $0.58 \mathrm{eV}$, while it is $0.5 \mathrm{eV}$ at $\sim 375 \mathrm{~K}$. The values of $\mathrm{RbMgF}_{3}$ compare well with the values obtained for the $\mathrm{NaMgF}_{3}: 0.2 \% \mathrm{Mn}$ and $\mathrm{RbMgF}_{3}: 0.2 \% \mathrm{Eu}$. Potential additional peaks above $620 \mathrm{~K}$ could not be detected due to the temperature limitation of the equipment used. 


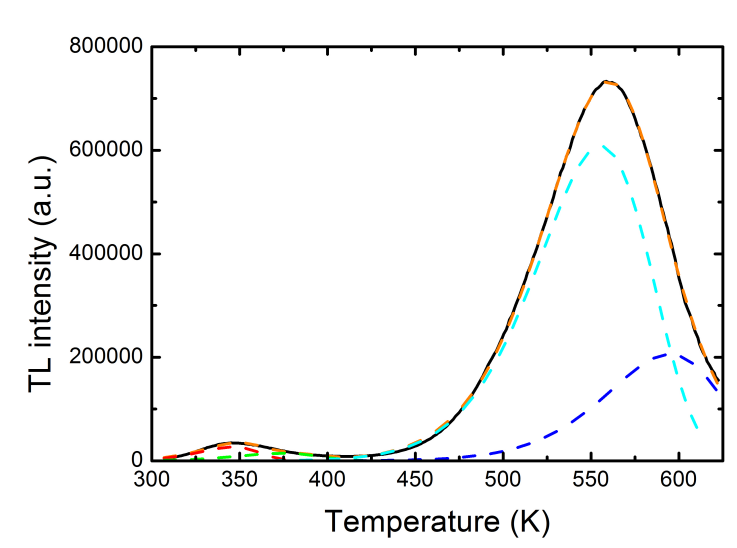

(a)

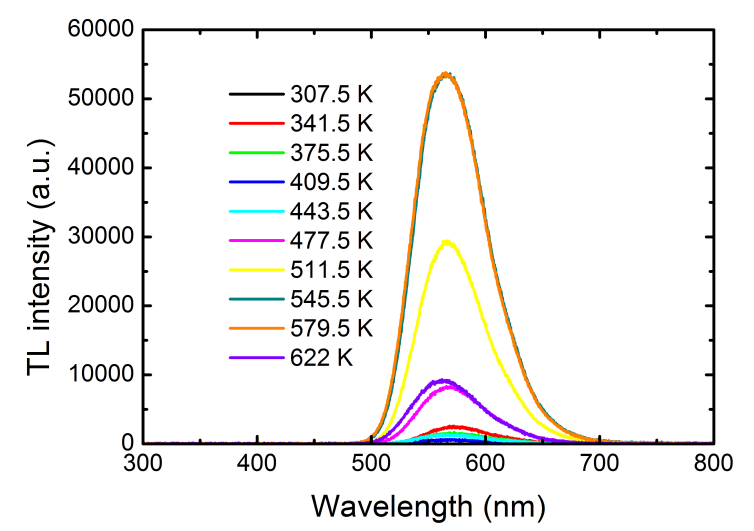

(b)

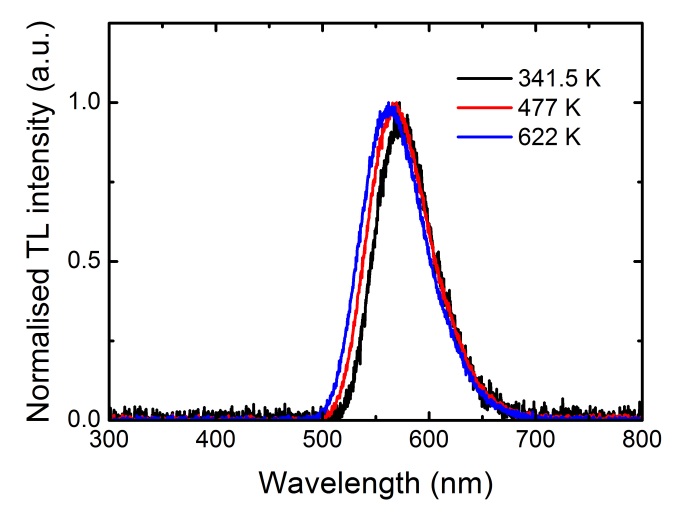

(c)

Figure 7.7: (a) TL for the $\mathrm{RbMgF}_{3}$ sample doped with $0.2 \% \mathrm{Mn}$ (solid curve). The TL signal was detected with the PMT after 1 min X-ray irradiation $(40 \mathrm{kV}, 40$ $\mathrm{mA}$ ) at a heating rate of $1 \mathrm{~K} / \mathrm{s}$. The fitted peaks were obtained using first order kinetics glow curve deconvolution (dashed curves).

(b) TL spectra vs. wavelength for the different temperatures detected with the CCD after 5 min X-ray irradiation.

(c) Normalised TL spectra at $341.5 \mathrm{~K}, 477 \mathrm{~K}$ and $622 \mathrm{~K}$.

Fig. 7.7(c) shows the normalised TL emission at $341.5 \mathrm{~K}, 477 \mathrm{~K}$ and $622 \mathrm{~K}$. For higher temperatures the peak maximum shifts towards higher energies and the peak is broader, which was also observed for the $\mathrm{NaMgF}_{3}$ bulk materials (see Fig. 5.9(c)). This phenomenon 
is similar to the temperature dependence of the band gap of semiconductors. This means that thw band gap increases with increasing temperature [87, 88, 89, 90].

Table 7.4: The activation energies of individual glow curve peaks for the $\mathrm{RbMgF}_{3}: 0.2 \%$ Mn sample obtained by first order kinetics glow curve deconvolution. The top line is the peak temperature for the glow curve.

\begin{tabular}{|l|l|l|l|l|}
\hline & $346 \mathrm{~K}$ & $375 \mathrm{~K}$ & $555 \mathrm{~K}$ & $595 \mathrm{~K}$ \\
\hline \hline $0.2 \%$ & $0.58 \pm 0.06 \mathrm{eV}$ & $0.50 \pm 0.05 \mathrm{eV}$ & $0.89 \pm 0.09 \mathrm{eV}$ & $0.78 \pm 0.08 \mathrm{eV}$ \\
peak height & 26500 & 14600 & 608100 & 205500 \\
\hline
\end{tabular}

\subsection{Radioluminescence and temperature dependent $R L$ measurements}

Radioluminescence and temperature dependent RL measurements were performed with the same methods as described in section 5.3.1 for the measurements on $\mathrm{NaMgF}_{3}$.

\subsubsection{Radioluminescence measurements}

\subsubsection{Eu doped polycrystalline $\mathrm{RbMgF}_{3}$}

RL spectra of $\mathrm{RbMgF}_{3}$ doped with $\mathrm{Eu}$ are shown in Fig. 7.8. The RL intensity against wavelength is plotted in Fig. 7.8(a), while the RL intensity against dose is shown in Fig. 7.8(b). The RL spectra during continual X-ray excitation can be attributed to $\mathrm{Eu}^{2+}$ and $\mathrm{Eu}^{3+}$ emission. A broad peak around $420 \mathrm{~nm}$ was detected and its intensity increases with increasing dose. The energy of the $\mathrm{Eu}^{2+}$ emission is the same as observed in the PL spectra. Additional emission bands from $\mathrm{Eu}^{3+}$ ions were detected and the emission wavelengths remain unchanged (Fig. 7.8(a)). No sign of $\mathrm{Eu}^{3+}$ ions was detected in the 
PL spectra, most likely because of the intensity of the broad peak around $470 \mathrm{~nm}$ was too high. The integrated RL intensity is plotted for $\mathrm{Eu}^{2+}$ (filled squares) and $\mathrm{Eu}^{3+}$ (open squares) in Fig. 7.8(b). $\mathrm{I}_{R L}$ for $\mathrm{Eu}^{2+}$ increases by $48.8 \%$ and reaches a maximum after a dose of $211 \mathrm{~Gy}$. After this initial increase the RL intensity starts to decrease. After a dose of $12 \mathrm{KGy}$ it has been decreased by $73.9 \%$ at $12 \mathrm{kGy}$. This results in a total difference of $61.1 \%$ between the start point and the end point. The RL intensity is initially low because some of the X-ray generated electrons and holes become trapped and hence the amount of electrons and holes available for RL is low. After a high enough dose, all the traps will be filled and hence all the X-ray generated electrons and holes are available for RL. Thus, the RL will increase with dose until all the electron and hole traps are filled. This explains the initial increase in the RL (Fig. 7.8(c)). The RL starts to decrease at high doses due to X-ray induced radiation damage that results in non-radiative recombination sites. Thus, as shown in Fig. 7.8(d), some of the X-ray generated electrons and holes are not available for RL due to non-radiative recombination, which leads to a reduction of the $\mathrm{RL}$ at high doses. This non-radiative centre competes with Eu as recombination centre and the probability for mobile carriers to recombine in this centre is higher compared to Eu. On the other hand an increase with $55.8 \%$ after a dose of 211 Gy and a further increase with $87.2 \%$ after $12 \mathrm{kGy}$ was observed for the $\mathrm{I}_{R L}$ of the $\mathrm{Eu}^{3+}$ ions. The RL spectra contains a broad band between $400 \mathrm{~nm}$ and $500 \mathrm{~nm}$. This band can probably be assigned to a defect of unknown origin. During irradiation the intensity of this band increases and the maximum shifts from $400 \mathrm{~nm}$ towards $420 \mathrm{~nm}$. The integrated radioluminescence intensity increases by $86.5 \%$ after a dose of $1 \mathrm{kGy}$ and by $155 \%$ after a large dose of $12.7 \mathrm{kGy}$ (Fig. 7.8(b)). This behaviour is different than previously observed for $\mathrm{NaMgF}_{3}$. In $\mathrm{NaMgF}_{3}$ the RL intensity of the unknown defect decreased with dose as demonstrated in section 5.3.1 and shown in Fig. 5.13(b). 


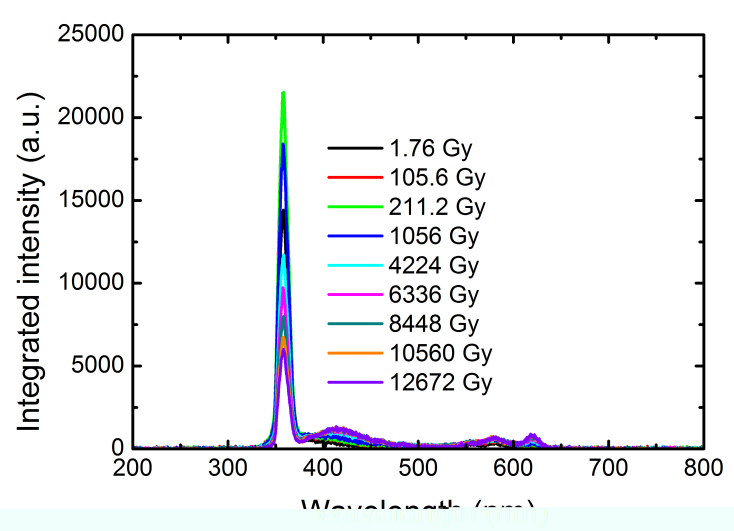

(a)

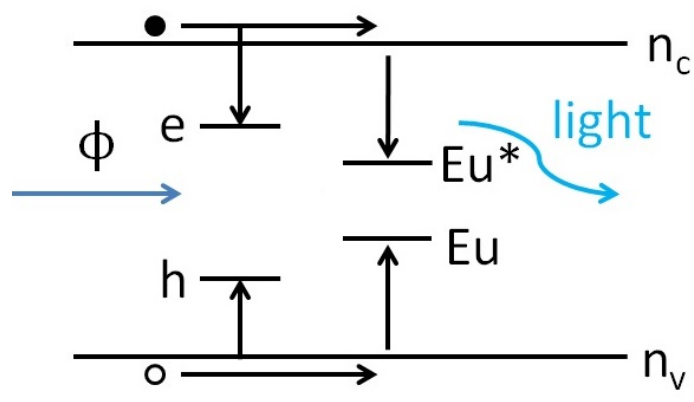

(c)

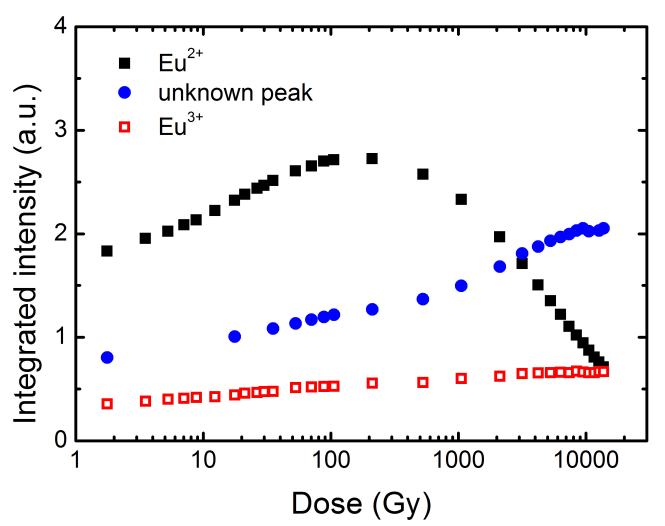

(b)

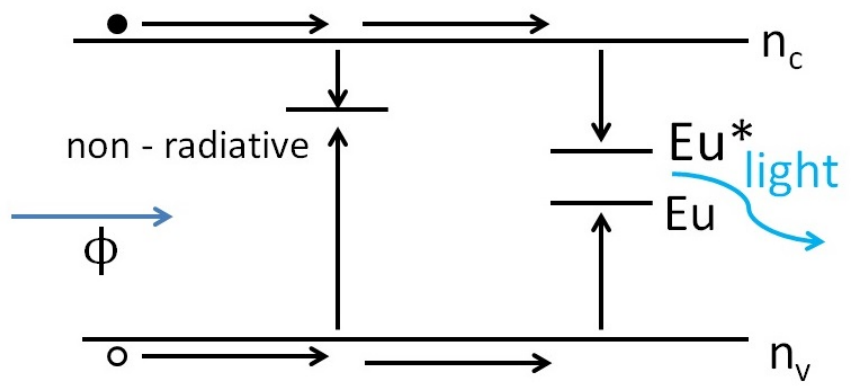

(d)

Figure 7.8: (a) Radioluminescence spectra for $\mathrm{RbMgF}_{3}: 0.2 \% \mathrm{Eu}$ versus the dose and

(b) the integrated RL spectra as a function of dose during continuous X-ray irradiation $\left(\mathrm{Eu}^{2+}\right.$ : filled squares, $\mathrm{Eu}^{3+}$ : open squares, unknown defect: circles).

(c) Existence of electron and hole traps next to the Eu.

(d) Dose dependence of the RL signal as a result of the generation of nonradiative recombination centres.

Fig. 7.9 shows the emission spectra of the unknown defect centred at $470 \mathrm{~nm}$ for the PL, TL and RL measurements. The PL spectrum was excited at $396 \mathrm{~nm}$, the TL spectrum was measured at a temperature of $620 \mathrm{~K}$ and the RL was measured using an X-ray excitation and after an initial dose of $12 \mathrm{kGy}$. The emission wavelengths of the unknown defect are different for every measurement. The PL spectra consists out of two maxima. The first around $455 \mathrm{~nm}$ and the second at $505 \mathrm{~nm}$. On the other hand the TL spectra consists out 
of a broad peak around $580 \mathrm{~nm}$ and a shoulder at $420 \mathrm{~nm}$. While the RL spectra show a broad peak centred at $420 \mathrm{~nm}$, and a smaller one at around $560 \mathrm{~nm}$. From the differences of these spectra it seems that the unknown defect has different defect distributions. For the RL and TL the probability of the mobile carriers to get trapped in other defects is higher Fig. 7.9(b). Thus, the unknown peak observed in the PL spectra will not necessarily be detected in the RL and TL spectra (Fig. $7.9(\mathrm{a}+\mathrm{b})$ ).

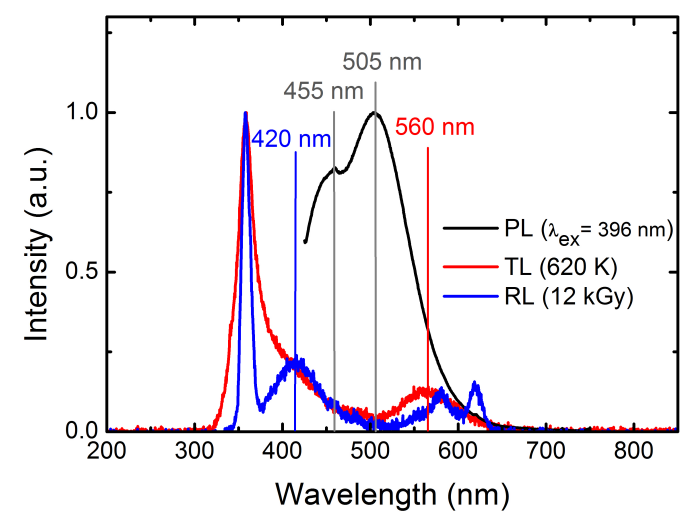

(a)

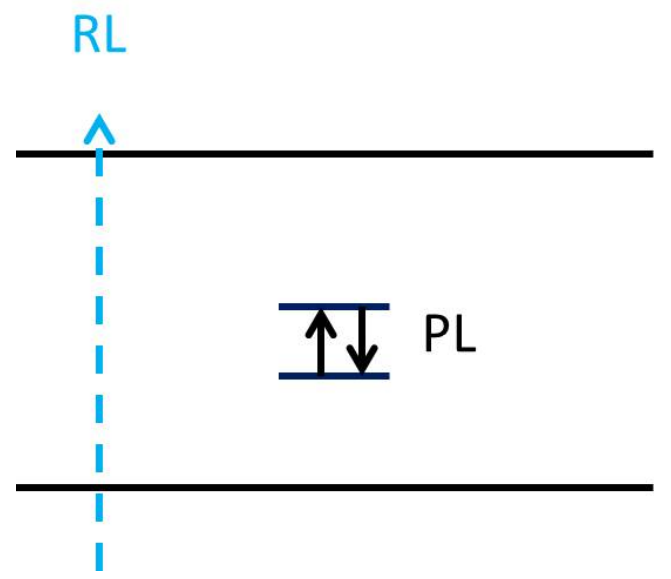

(b)

Figure 7.9: (a) PL, TL and RL emission spectra for the broad peak around $400 \mathrm{~nm}$ to 580 $\mathrm{nm}$.

(b) Different excitation regions for PL and RL.

\subsubsection{Mn doped polycrystalline $\mathbf{R b M g F}_{3}$}

The $\mathrm{Mn}$ doped polycrystalline sample of $\mathrm{RbMgF}_{3}$ was also irradiated continuously up to a dose of 12.7 kGy. Fig. 7.10(a) shows the RL emission spectra plotted against the wavelength for different doses, while Fig. 7.10(b) shows the dose dependence of the integrated RL intensity. The luminescence in the RL spectra of this sample can be completely attributed to $\mathrm{Mn}^{2+}$ with an emission band at 580nm. There is no spectral change of the RL or PL emission after heavy irradiation. Fig. 7.10(b) shows the integrated RL intensity plotted against the X-ray dose and the RL increases by $125.4 \%$ at $\sim 1 \mathrm{kGy}$ and afterwards 
decreases by $9.4 \%$ at $12 \mathrm{kGy} . \mathrm{NaMgF}_{3}$ : Mn showed also an increase of the RL signal with a doses up to $8.5 \mathrm{kGy}$ and then the signal would decrease (Fig. 5.14(b)). The behaviour of the RL signal can be explained with the same model as used previously for the Eu doped samples (see Fig. 7.8 $(\mathrm{c}+\mathrm{d})$ ). The RL intensity is initially low because some of the X-ray generated electrons and holes become trapped and hence electrons and holes available for RL are low. For a high enough dose, all of the traps are filled and hence all of the X-ray generated electrons and holes are available for RL. Thus, the RL will increase with dose until all of the electron and hole traps are filled. This explains the initial increase in the RL until 1 kGy Fig. 7.8(c). The decrease of the RL signal for doses above $1 \mathrm{kGy}$ is due to $\mathrm{X}$-ray induced radiation damage that results in non-radiative recombination sites. Thus, as shown in Fig. 7.8(d), some of the X-ray generated electrons and holes are not available for $\mathrm{RL}$ due to non-radiative recombination, which leads to a reduction in the RL for high doses. Additionally, there could be a conversion of $\mathrm{Mn}^{2+} \rightarrow \mathrm{Mn}^{3+}$. No $\mathrm{Mn}^{3+}$ was detected in the PL spectra, but this might be due to the signal of $\mathrm{Mn}^{3+}$ being too low.

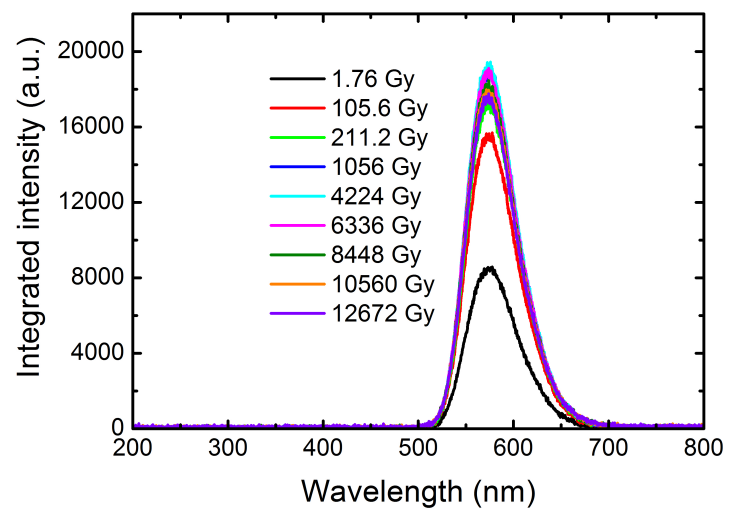

(a)

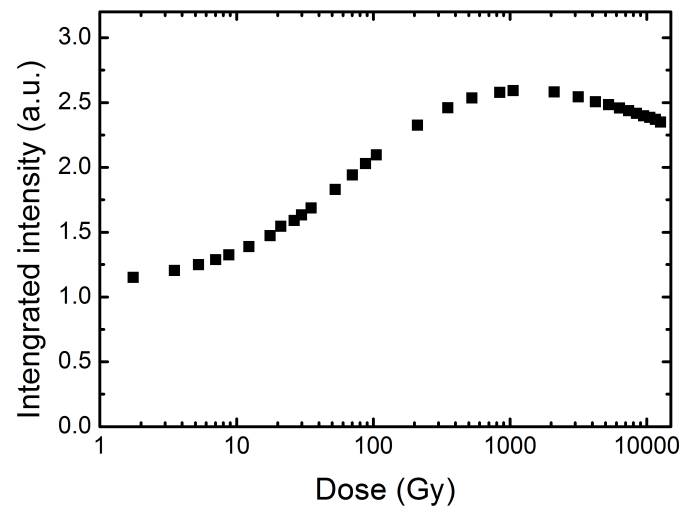

(b)

Figure 7.10: (a) Radioluminescence spectra for $\mathrm{RbMgF}_{3}: 0.2 \% \mathrm{Mn}$ for versus the dose and (b) the integrated RL spectra as a function of dose during continuous X-ray irradiation. 


\subsubsection{Temperature dependent RL measurements}

Temperature dependent RL measurements were performed on powdered samples using a method similar as previously mentioned for $\mathrm{NaMgF}_{3}$ in section 5.3.2.

\subsubsection{Eu doped polycrystalline $\mathrm{RbMgF}_{3}$}

The temperature dependent measurements were done by powdering the samples, which were saturated with isopropanol, on a ceramic holder and heating the holder up until a certain temperature. After the temperature had stabilized RL measurements up to a dose of 15 Gy were performed. Then the sample was thermally bleached by heating it up until 350 ${ }^{\circ} \mathrm{C}$. This bleaching empties shallow traps as previously observed in the $\mathrm{NaMgF}_{3}$ samples. Hence, future measurements should have been performed with an optical bleaching step after each temperature change. This process was repeated for 13 different temperature settings. The total dose the sample received at the end was $\sim 200 \mathrm{~Gy}$.

The $\mathrm{RbMgF}_{3}$ sample shows a decrease in the $\mathrm{Eu}^{2+} \mathrm{RL}$ compared to room temperature when the temperature is increased to $100{ }^{\circ} \mathrm{C}$. The initial decrease in the RL with increasing temperature may be due to thermal activation of trapped charges to non-radiative recombination centres as shown in Fig. 7.11(c). The appearance of RL does not depend on the filling of the traps. If the temperature increases, the probability of hopping increases. This means that the carriers overcome an energy barrier, $\Delta$, and could recombine in non-radiative recombination centres (Fig. 7.11(c)). For small doses and hence a negligible trapped carrier concentration, the RL signal is expected to decrease with decreasing temperature, which is not observed. Thus, it is likely that the dose at each temperature results in a significant carrier trapping. Therefore, as the temperature decreases there may be competition between reducing the RL through reducing the thermal detrapping probability and increasing the RL due to significant trap filling at each temperature. It is apparent that the second process dominates in Fig. 7.11(a). The same process was also shown in Fig. 5.16(a) for the $\mathrm{NaMgF}_{3}$ polycrystalline materials. The thermal coefficient of 
the RL was calculated using the average for each temperature dependent RL intensity, and was around $0.13 \pm 0.1 \% / \mathrm{K}$. There was no observable shift in the RL emission wavelengths of $\mathrm{Eu}^{2+}$ transitions with increasing temperature because the temperature change was too low.

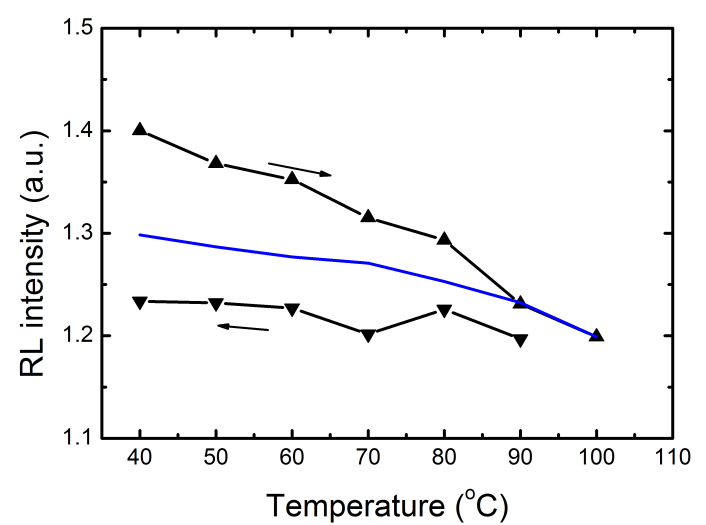

(a)

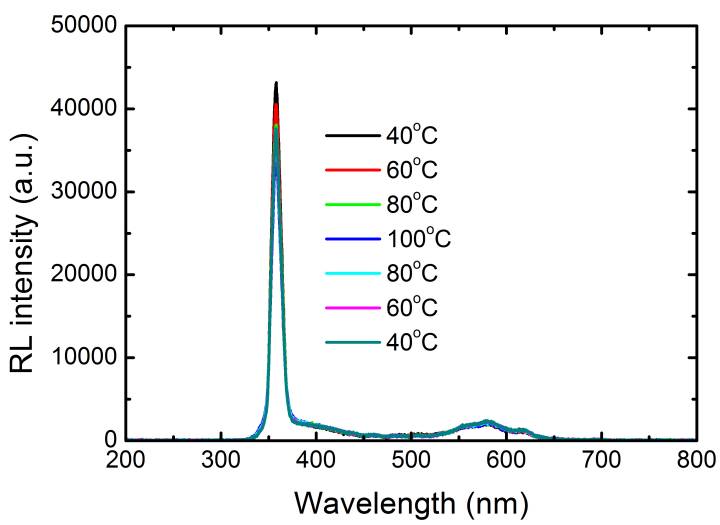

(b)

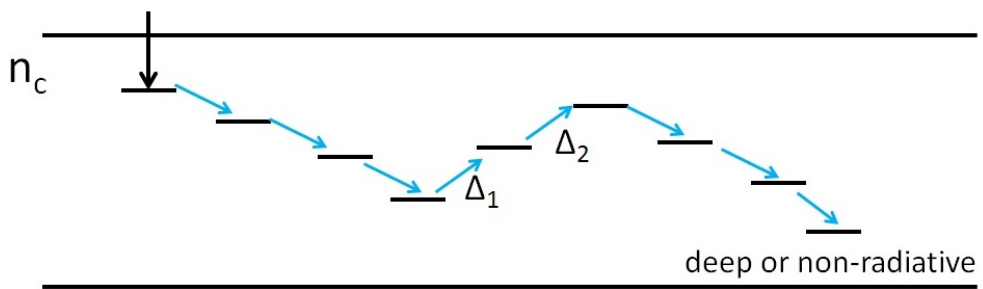

$\mathrm{m}_{\mathrm{v}}$

(c)

Figure 7.11: (a) Temperature dependent RL for $\mathrm{RbMgF}_{3}: 0.2 \% \mathrm{Eu}$ for different temperature up to $100{ }^{\circ} \mathrm{C}$. Arrows facing up signal an increase of temperature, and arrows facing down signal a decrease in temperature.

(b) RL spectra measured at different temperatures.

(c) Hopping of the carriers to a deep non-radiative trap due to increasing temperature. 


\subsubsection{Mn doped polycrystalline $\mathrm{RbMgF}_{3}$}

Fig. 7.12 shows the temperature dependent RL intensity for the Mn doped polycrystalline $\mathrm{RbMgF}_{3}$ sample. The RL signal shows a similar temperature dependent behaviour as previously discussed for the Eu doped samples. At low temperatures, the traps become partially filled but they will not be completely filled after a high enough dose. The shape of the temperature dependent RL intensity does not depend on the filling of the traps. With increasing temperature the probability of hopping increases. This means that the carriers overcome an energy barrier, $\Delta$, and could recombine in non-radiative recombination centres as shown in Fig. 7.11(c). For small doses and hence a negligible trapped carrier concentration, the RL signal should decrease with decreasing temperature. The opposite was observed. It is likely that the dose at each temperature results in a significant carrier trapping. As the temperature is reduced, there will be a competition between reducing the RL through reducing the thermal detrapping probability and increasing the RL due to significant trap filling at each temperature. It is apparent that the second process dominates in Fig. 7.12(a). The same process was also shown in Fig. 5.16(a) for the $\mathrm{NaMgF}_{3}$ polycrystalline materials. The thermal coefficient of the RL was calculated using the average for each temperature dependent RL curve, and was around $0.06 \pm 0.1 \% / \mathrm{K}$. For the $\mathrm{NaMgF}_{3}$ doped with $\mathrm{Mn}$ the RL signal increases with increasing temperature and decreases for measurements from $100{ }^{\circ} \mathrm{C}$ to $40{ }^{\circ} \mathrm{C}$. There was no observable shift in the $\mathrm{RL}$ emission wavelengths of $\mathrm{Mn}^{2+}$ transitions with increasing temperature because the temperature change was too low. 


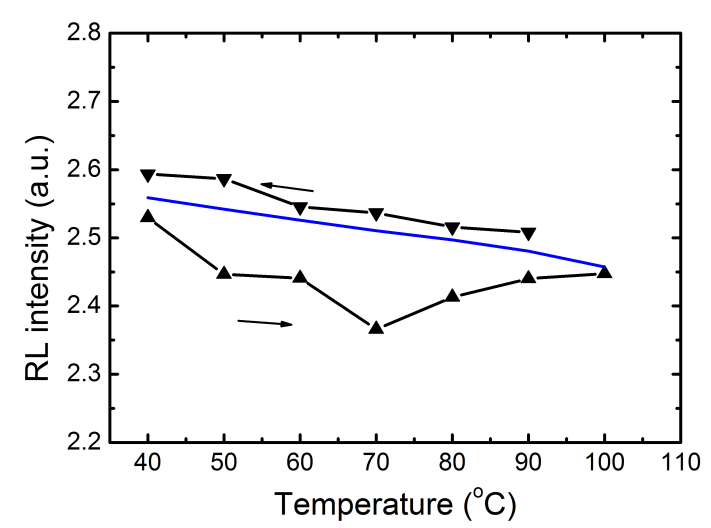

(a)

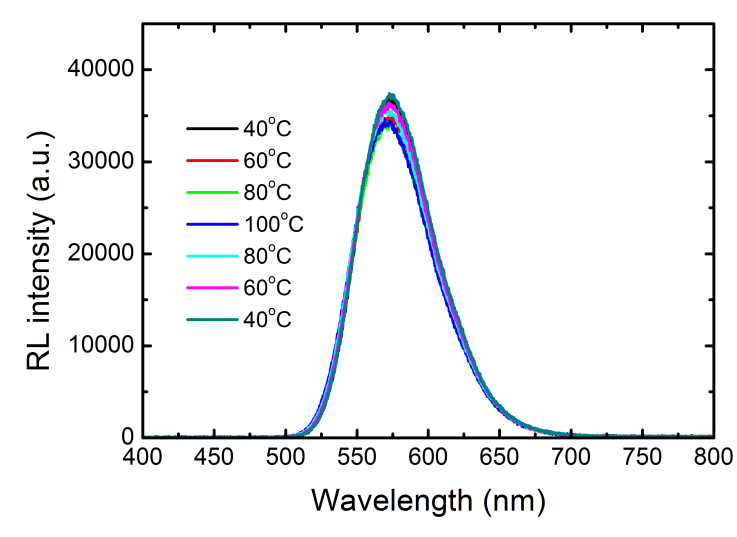

(b)

Figure 7.12: (a) Temperature dependent RL for $\mathrm{RbMgF}_{3}: 0.2 \% \mathrm{Mn}$ for different temperature up to $100{ }^{\circ} \mathrm{C}$. Arrows facing up signal an increase of temperature, and arrows facing down signal a decrease in temperature.

(b) RL spectra measured for different temperatures.

\subsection{Conclusion}

PL, TL and RL were observed for polycrystalline samples of $\mathrm{RbMgF}_{3}$ doped with Eu or Mn. Photoluminescence measurements show the presence of $\mathrm{Eu}^{2+}$ ions in the Eu doped samples. PL measurements of the Eu doped samples before and after heavy irradiation show that the total intensity of the $\mathrm{Eu}^{2+}$ emission decreases, but the peak intensity of the unknown defect increases. The measured lifetimes for the sharp emission band of $\mathrm{Eu}^{2+}$ located at $360 \mathrm{~nm}$ is higher for the $\mathrm{RbMgF}_{3}$ samples compared to the $\mathrm{NaMgF}_{3}$ samples. This is because there are less non-radiative recombination sites in the $\mathrm{RbMgF}_{3}$ crystal. Further work should be performed to find the origin of the unknown peak and if it is a F-centre or other defects. One possibility could be, to measure the sample with electron paramagnetic resonance (EPR). If the unknown peak is dominated by F-centres, it would be possible to detect this with EPR [98]. Another possibility would be, to powder the sample and heat it up. Oxygen will diffuse through most of the sample when the particles 
are small comparable to the oxygen diffusion mean free path. If afterwards an increase in the PL intensity is observed, it would mean that these defects are caused by oxygen. Oxygen can also be detected by performing vacuum UV-PL on the peak. TL measurements on the Eu samples show that the defects are different compared to the $\mathrm{NaMgF}_{3}$ : Eu bulk materials TL peaks. TL was observed for the unknown defect centred at $400 \mathrm{~nm}$ to 560 $\mathrm{nm}$ for $\mathrm{RbMgF}_{3}$ sample. The calculated activation energies for the unknown defect were similar to the $\mathrm{Eu}^{2+}$ ones. There was no TL observed for the unknown defect observed in the $\mathrm{NaMgF}_{3}$ samples. The RL intensity of the unknown defect decreases with dose for the $\mathrm{NaMgF}_{3}$ samples but it increases with dose for the $\mathrm{RbMgF}_{3}$ samples. The thermal coefficient of RL is $0.13 \pm 0.1 \% / \mathrm{K}$ for $\mathrm{RbMgF}_{3}: 0.2 \%$.

Photoluminescence measurements show the presence of two regular sites of $\mathrm{Mn}^{2+}$. There was no presence of a peak around $500 \mathrm{~nm}$ from a distorted $\mathrm{Mn}^{2+}$ site as was observed for manganese doped $\mathrm{NaMgF}_{3}$. The two $\mathrm{Mn}^{2+}$ sites in $\mathrm{RbMgF}_{3}$ show different average lifetimes, a higher lifetime for the site at $550 \mathrm{~nm}$ and a lower one for the $\mathrm{Mn}^{2+}$ site at 580 $\mathrm{nm}$. This might be due to a lower concentration of non-radiative recombination sites at $\mathrm{Mn}^{2+}$ site at $580 \mathrm{~nm}$, or due to the different symmetry caused by the different crystal field. EPR measurements should also be performed on $\mathrm{RbMgF}_{3}$ : Mn to observe the decrease of the RL above $1 \mathrm{kGy}$, and to investigate if it is caused from a conversion from $\mathrm{Mn}^{2+}$ $\rightarrow \mathrm{Mn}^{3+}$ by irradiation [98]. The RL signal of the $\mathrm{RbMgF}_{3}$ : Mn has a similar behaviour to $\mathrm{NaMgF}_{3}$ : Mn bulk materials, and it can be assumed that the defects have the same origin. The thermal coefficient of $\mathrm{RL}$ is $0.06 \pm 0.1 \% / \mathrm{K}$ for $\mathrm{RbMgF}_{3}: 0.2 \% \mathrm{Mn}$. The temperature dependence of the RL mainly arises from deep traps, and thermal bleaching is not enough to empty them. Therefore, future measurements should be performed with optical bleaching after each temperature change. 


\section{$8 \mathrm{RbMgF}_{3}$ nanoparticles}

The nanoparticles were either doped with $0.1 \%, 0.5 \%, 1 \%, 2 \%$ and $5 \%$ Eu or $1 \%$, $2 \%$ and $5 \% \mathrm{Mn}$. The sample preparation was already mentioned in chapter 4.1.2. The crystal structure is hexagonal crystal structure with the space group $P_{6_{3} / m m c}$ and from XRD measurements, the crystal size was calculated with the Scherrer formula from the XRD spectra shown in Fig. 4.1, which was $\sim 25 \mathrm{~nm}$. To perform PL, PL lifetime and RL measurements, the nanoparticles were also pressed into pellets with a diameter of $\sim 6$ $\mathrm{mm}$ and a thickness between $0.5 \mathrm{~mm}$ and $1.5 \mathrm{~mm}$. The appearance of the pressed pellets was whitish. The influence of the concentration of the dopant on the PL spectra, PL lifetime, TL and RL measurements will be investigated. There was no OSL observed for the nanoparticles.

\subsection{Photoluminescence and PL lifetime measurements}

PL measurements were performed with the Jobin-Yvon FluoroLog ${ }^{\circledR}$. The spectra were all normalised to the maximum.

\subsubsection{Eu doped $\mathrm{RbMgF}_{3}$ nanoparticles}

Fig. 8.1 shows the PL excitation spectra (a) and emission spectra (b) for $\mathrm{RbMgF}_{3}$ doped with Eu. The PL excitation spectra was measured with an emission wavelength of 360 
$\mathrm{nm}$ for the $\mathrm{Eu}^{2+}$ ions (dashed curve) and with an emission wavelength of $592 \mathrm{~nm}$ for the transition from ${ }^{7} \mathrm{~F}_{1}$ level of the $\mathrm{Eu}^{3+}$ ion (Fig. 8.1(a)). In Fig. 8.1(b), the PL emission spectra for the $\mathrm{Eu}^{3+}$ ion of the $1 \%$ (dashed curve) and $5 \%$ doped sample (solid curve) are plotted. The spectra of the $\mathrm{Eu}^{3+}$ site consists of the ${ }^{5} \mathrm{D}_{1}$ and ${ }^{5} \mathrm{D}_{0}$ transitions, which are indicated in Fig. 8.1. The ratio ${ }^{7} \mathrm{~F}_{1} /{ }^{7} \mathrm{~F}_{2}$ decreases from $1.4: 1$ for the $1 \%$ sample to 0.8:1 for the $5 \%$ sample. An unknown defect centred at $470 \mathrm{~nm}$ was detected. The PL intensity of the broad defect remains unchanged for the measurements of the $1 \%$ sample and the $5 \%$ Eu sample as shown in Fig. 8.1(b). The PL intensity of the unknown defect in the $\mathrm{NaMgF}_{3}$ :Eu nanoparticles decreased with increasing $\mathrm{Eu}^{3+}$ concentration. The inset in Fig. 8.1(b) shows the PL emission while excited at $256 \mathrm{~nm}$. The PL emission mainly arises from the transitions of ${ }^{6} \mathrm{P}_{7 / 2}$ and ${ }^{6} \mathrm{P}_{7 / 2}$ to the ground state, ${ }^{8} \mathrm{~S}_{7 / 2}$ - sharp peak centred at $\lambda_{e m}=360 \mathrm{~nm}$. The broad peak centred at $380 \mathrm{~nm}$ arises from transitions from the $\mathrm{E}_{g}$ level to the ${ }^{8} \mathrm{~S}_{7 / 2}$ ground state.

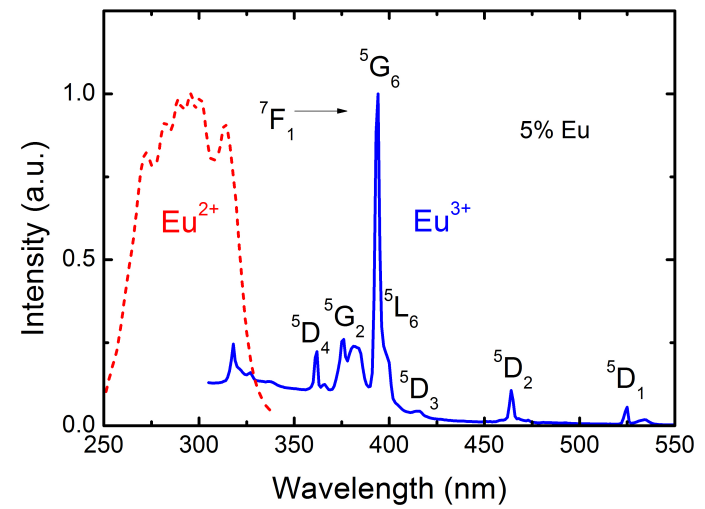

(a)

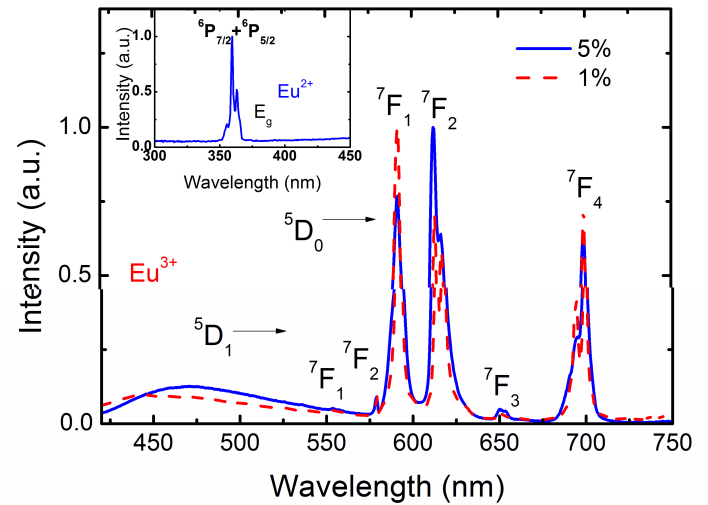

(b)

Figure 8.1: (a) PL excitation for the $\mathrm{Eu}^{2+}\left(\lambda_{e m}=360 \mathrm{~nm}\right.$ - dashed curve $)$ and $\mathrm{Eu}^{3+}\left(\lambda_{e m}=\right.$ $592 \mathrm{~nm}-$ solid curve) and (b) emission ( $\lambda_{e x}=396 \mathrm{~nm}$; inset $\lambda_{e x}=256 \mathrm{~nm}$ ) spectra for $\mathrm{RbMgF}_{3}$ doped with $1 \% \mathrm{Eu}$ (dashed curve) and $5 \% \mathrm{Eu}$ (solid curve).

Temperature dependent PL measurements of $\mathrm{RbMgF}_{3}$ : Eu doped pellets were performed 
and the PL plots of the $0.1 \%$ (a), $1 \%$ (b) and $5 \%$ (c) samples are shown in Fig. 8.2. The solid curves show measurement performed at $10 \mathrm{~K}$ and the dashed curves were measured at $300 \mathrm{~K}$. The insets in Fig. 8.2 show the PL spectra detected for an emission wavelength at $450 \mathrm{~nm}$ at $10 \mathrm{~K}$ (red curve) and $300 \mathrm{~K}$ (black curve). The $0.1 \% \mathrm{Eu}$ doped $\mathrm{RbMgF}_{3}$ sample shows a shoulder centred at $450 \mathrm{~nm}$ and a broad peak centred at $550 \mathrm{~nm}$ for the measurements at $10 \mathrm{~K}$. The transitions from $\mathrm{Eu}^{3+}$ were quenched by the higher intensity of the broad defect centred at $550 \mathrm{~nm}$ (Fig. 8.2(a)). The unknown defect with a peak centred at $550 \mathrm{~nm}$ disappears for measurements at $300 \mathrm{~K}$. Therefore, $\mathrm{Eu}^{3+}$ transitions can be detected as well as an unknown defect centred at $470 \mathrm{~nm}$ (Fig. 8.2(a)). The inset in Fig. 8.2(a) shows the PL excitation spectrum with an emission wavelength at $450 \mathrm{~nm}$. In the PL spectrum consists of two peaks, one centred at $290 \mathrm{~nm}$ and another at 400 $\mathrm{nm}$ for measurements at $10 \mathrm{~K}$. These peaks broaden and shift towards higher energies for measurements at $300 \mathrm{~K}$.

For the $0.5 \%$ doped sample, a shoulder centred at $450 \mathrm{~nm}$, an additional unknown defect with centred at $\sim 550 \mathrm{~nm}$ and $\mathrm{Eu}^{3+}$ transitions were detected (Fig. 8.2(b)). Peak intensities from the emission below $570 \mathrm{~nm}$ decrease with increasing temperature. In the inset of Fig. 8.2(b), the PL was measured for an emission wavelength at $450 \mathrm{~nm}$ and the spectrum consists of two broad peaks, one centred at $\sim 290 \mathrm{~nm}$ and another one at $\sim 400 \mathrm{~nm}$ for measurements at $10 \mathrm{~K}$. The intensity of the first peak centred at $\sim 290 \mathrm{~nm}$ decreases for measurements at room temperature.

The $1 \%$ Eu doped pellets show only one broad peak with a maximum at $470 \mathrm{~nm}$ at $10 \mathrm{~K}$. The PL intensity of this unknown defect centred at $\sim 470 \mathrm{~nm}$ decreases for measurements at room temperature (Fig. 8.2(c)). The inset in this graph shows the PL excitation spectra, which is detected at $450 \mathrm{~nm}$ for both temperatures at $10 \mathrm{~K}$ and $300 \mathrm{~K}$. The spectra consists of two broad peaks, one centred at $\sim 290 \mathrm{~nm}$ and another one at $\sim 370 \mathrm{~nm}$. The intensity of the peak centred at $\sim 300 \mathrm{~nm}$ decreases with increasing temperature. The centre of the peak of these unknown defects shift to lower wavelengths with increasing temperature (inset Fig. $8.3(\mathrm{c}))$. The ${ }^{5} \mathrm{D}_{0} \rightarrow{ }^{7} \mathrm{~F}_{J}$ transitions of the $1 \%$ doped nanoparticles were detected 
for measurements at $10 \mathrm{~K}$ and $300 \mathrm{~K}$. Their normalised intensities are higher than the PL intensity of the unknown defect centred at $\sim 450 \mathrm{~nm}$ when compared to the $0.1 \%$ doped sample.

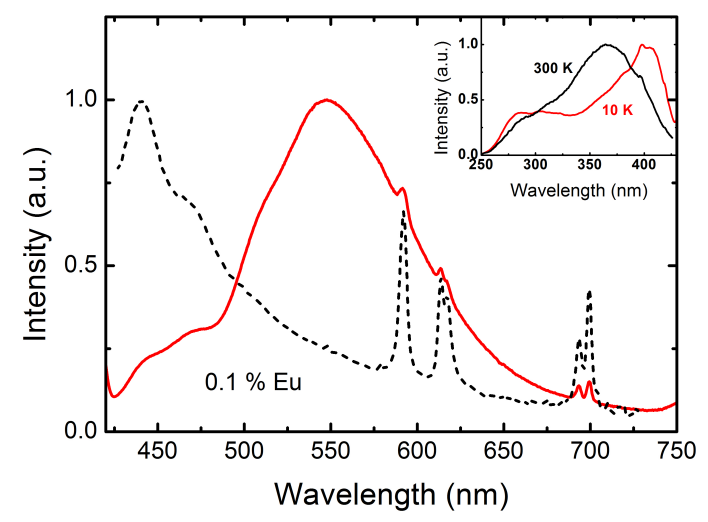

(a)

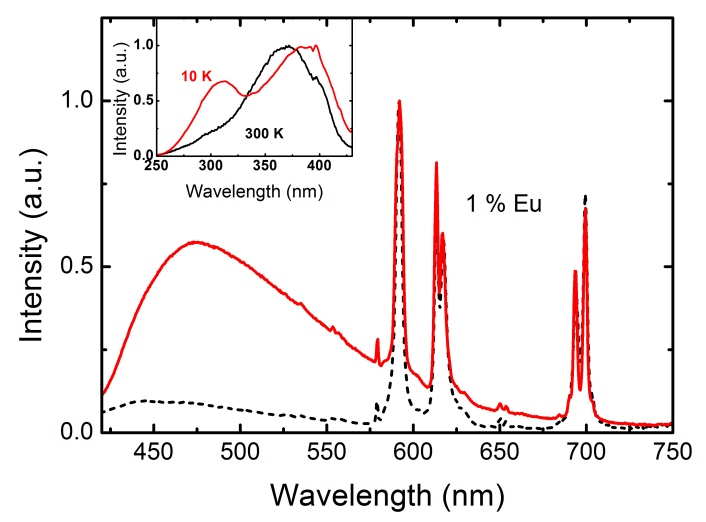

(c)

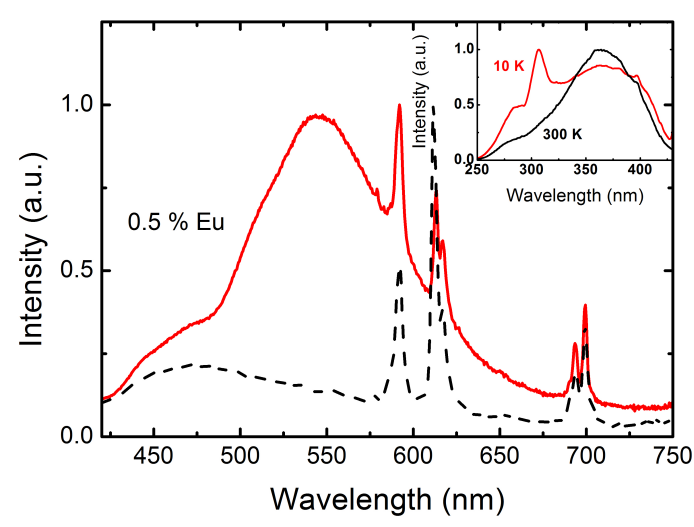

(b)

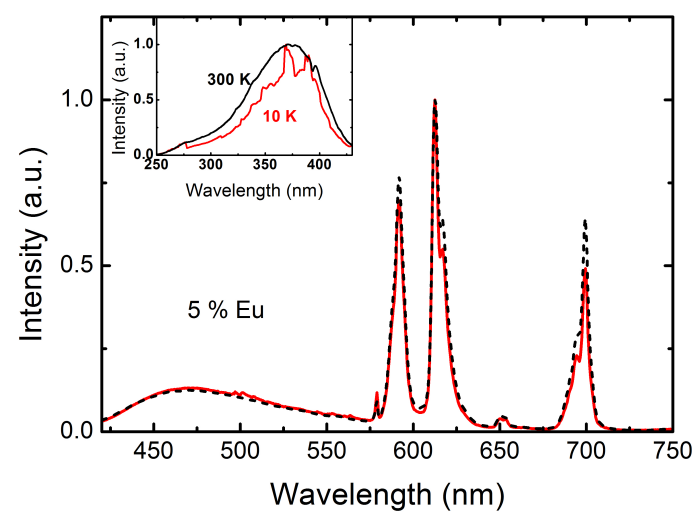

(d)

Figure 8.2: (a) PL emission spectra for $0.1 \%$, (b) $0.5 \%$, (c) $1 \%$ and (d) $5 \%$ Eu doped $\mathrm{RbMgF}_{3}$ at $\lambda_{e x}=396 \mathrm{~nm}$ at $10 \mathrm{~K}$ (solid curve) and at room temperature (dashed curve). Inset: PL excitation spectra detected at $\lambda_{e m}=450 \mathrm{~nm}$ for the different Eu concentrations of $\mathrm{RbMgF}_{3}$.

The nanoparticles doped with $5 \%$ Eu show an emission from the unknown defect centred at $\sim 450 \mathrm{~nm}$ and from ${ }^{5} \mathrm{D}_{0}$ transitions (Fig. $8.2(\mathrm{~d})$ ). The normalised PL intensity of the unknown defect decreases for measurements at room temperature resulting in an increase 
of the normalised PL intensity of the ${ }^{5} \mathrm{D}_{0}$ transition. The inset in Fig. 8.2(d) shows the PL excitation with an emission wavelength of $450 \mathrm{~nm}$. The peak at $\sim 290 \mathrm{~nm}$ disappears for higher Eu concentrations. However, the peak centred at $\sim 370 \mathrm{~nm}$ is broader for measurements at room temperature. For the $\mathrm{NaMgF}_{3}$ : Eu nanoparticles, a similar behaviour was observed. All of the samples show a double peak with maxima at $\sim 290 \mathrm{~nm}$ and 370 $\mathrm{nm}$ at $10 \mathrm{~K}$ and the intensity of the peak centred at $\sim 290 \mathrm{~nm}$ decreases for measurements at room temperature. The ratio $I_{{ }^{7} F_{2}} / \mathrm{I}_{7} F_{1}$ is $1.1: 1$ for the $0.1 \%$ sample, 1.9:1 for the 0.5 \% sample, 1:1 for the $1 \%$ Eu doped sample, 1.1:1 for the $2 \%$ doped sample and 1.6:1 for the $5 \%$ sample. This means the higher this ratio the more distorted is the average crystal environment of the $\mathrm{Eu}^{3+}$ ion.

The PL lifetimes for the $\mathrm{Eu}^{2+}$ ions were measured with the Cary 50. For $\mathrm{Eu}^{3+}$ and the unknown defect average PL lifetimes were measured with the Jobin-Yvon FluoroLog ${ }^{\circledR}$ spectrometer. The PL decay can be fitted with multiple exponential components and the average lifetime was estimated as already explained in section 3.2.4. $\tau_{\text {rad }}$ was calculated with the corrected emission spectra of the $\mathrm{Eu}^{3+}$ emission where signal from the broad unknown defect centred at $\sim 470 \mathrm{~nm}$ was subtracting. The temperature dependence of the PL lifetime is plotted in Fig. 8.3 for the $1 \%$ Eu doped sample and in Fig. 8.4 for the $5 \% \mathrm{Eu}$ doped nanoparticles. Fig. 8.3(a) shows the PL lifetime calculated of the three components $\tau_{1}, \tau_{2}$ and $\tau_{3}$. The first two components $\tau_{1}$ and $\tau_{2}$ seem to be less temperature dependent as $\tau_{3}$. Above $180 \mathrm{~K}$, the values are bigger than the radiative lifetime, $\tau_{\text {rad }}=$ $7.47 \pm 0.75 \mathrm{~ms}$. The values get up to $11.3 \pm 1.1 \mathrm{~ms}$ at $10 \mathrm{~K}$. Therefore, these values were not used for the calculation of $\tau_{12, \text { ave }}$ as already explained for the $\mathrm{NaMgF}_{3}$ : Eu nanoparticles. An average lifetime, $\tau_{12, a v e}$, of $3.14 \pm 0.31 \mathrm{~ms}$ was calculated at room temperature. The third component, $\tau_{3}$, might originate from the broad defect centred at $500 \mathrm{~nm}$. Hence, the calculated average PL lifetime for all three components is higher than $\tau_{\text {rad }}$ for $\mathrm{Eu}^{3+}$. 


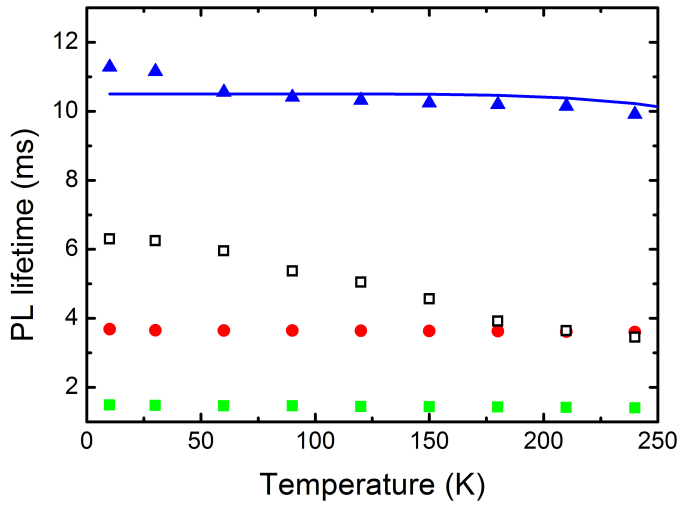

(a)

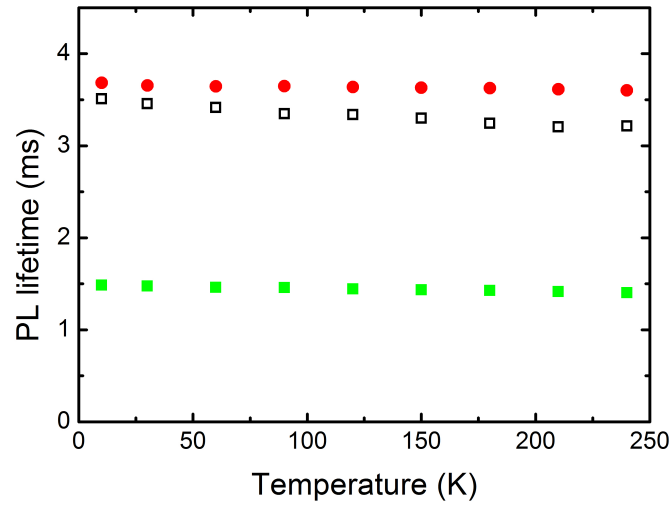

(b)

Figure 8.3: (a) Temperature dependent average PL lifetime of $1 \% \mathrm{Eu}$ doped $\mathrm{RbMgF}_{3}$ $\left(\lambda_{e m}=592 \mathrm{~nm} ; \lambda_{e x}=375 \mathrm{~nm}\right)$ from the three exponential fit of the data with only the $\tau_{1}$ (filled squares), $\tau_{2}$ (filled circles), $\tau_{3}$ (filled triangles) and the average lifetime, $\tau_{123, a v e}$ (open squares). $\tau_{3}$ was fitted using equation 8.1 .

(b) Temperature dependent average PL lifetime of $1 \%$ Eu doped $\mathrm{RbMgF}_{3}$ from the three exponential fit of the data with only the $\tau_{1}$ (filled squares), $\tau_{2}$ (filled circles) and the average lifetime, $\tau_{12, a v e}$ (open squares) when only using the first and the second component.

It was already shown for the $\mathrm{NaMgF}_{3}$ nanoparticles that the temperature dependence of $\tau_{3}$ might occur from thermal quenching resulting in two decays: One radiative and one thermally active non-radiative. Therefore, $\tau_{3}$ can be expressed with the following equation,

$$
\frac{1}{\tau_{3}}=\frac{1}{\tau_{3,0}}+f \times e^{-\frac{E_{A}}{k_{B} T}}
$$

with $\tau_{3,0}$ as the intrinsic effective decay time at $0 \mathrm{~K}, \mathrm{E}_{A}$ the activation energy, $\mathrm{k}_{B}$ the Boltzmann's constant and $f$ the attempt frequency [10]. At $592 \mathrm{~nm} \tau_{3,0}$ is $10.5 \pm 1.1 \mathrm{~ms}$ and $\mathrm{E}_{A}$ is $129 \pm 42 \mathrm{meV}$.

Fig. 8.4(a) shows the three components of the average PL lifetime decay plotted with a multi-exponential fit for the $5 \%$ doped nanoparticles. It is shown that the third component 
is bigger than the radiative lifetime, $\tau_{\text {rad }}=6.23 \pm 0.62 \mathrm{~ms} . \tau_{3}$ is as high as $9.9 \pm 1.0 \mathrm{~ms}$ at $10 \mathrm{~K} . \tau_{3}$ is also significant temperature dependent below $240 \mathrm{~K}$. Therefore, the average lifetime, $\tau_{12 \text {,ave }}$, was calculated with the first two components, $\tau_{1}$ and $\tau_{2}$, leading toa $\tau_{12 \text {,ave }}$ of $2.42 \pm 0.24 \mathrm{~ms}$ at room temperature.

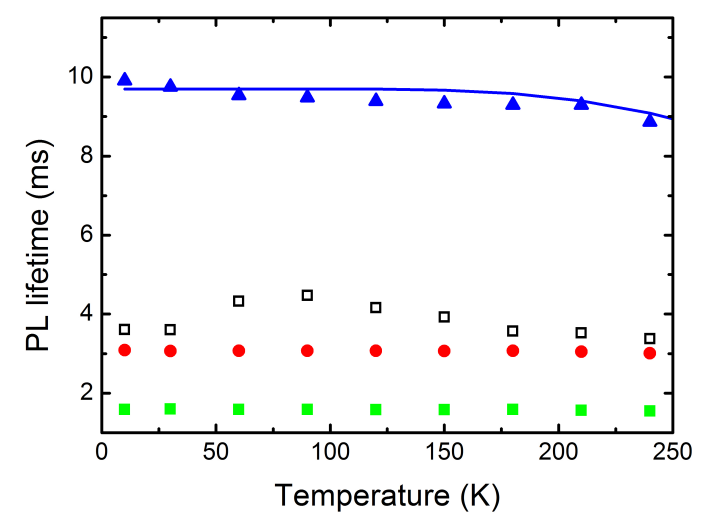

(a)

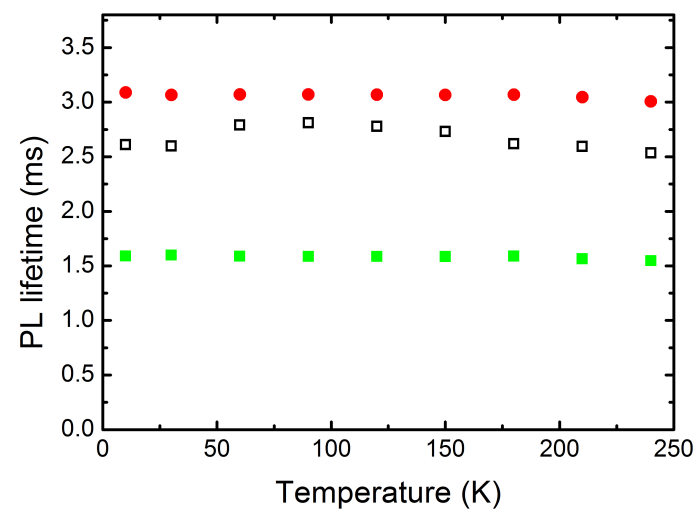

(b)

Figure 8.4: (a) Temperature dependent average PL lifetime of $5 \%$ Eu doped $\mathrm{RbMgF}_{3}$ $\left(\lambda_{e m}=592 \mathrm{~nm} ; \lambda_{e x}=375 \mathrm{~nm}\right)$ from the three exponential fit of the data with only the $\tau_{1}$ (filled squares), $\tau_{2}$ (filled circles), $\tau_{3}$ (filled triangles) and the average lifetime, $\tau_{123, \text { ave }}$ (open squares). $\tau_{3}$ was fitted using equation 8.1 .

(b) Temperature dependent average PL lifetime of $5 \%$ Eu doped $\mathrm{RbMgF}_{3}$ from the three exponential fit of the data with only the $\tau_{1}$ (filled squares), $\tau_{2}$ (filled circles) and the average lifetime, $\tau_{12, a v e}$ (open squares) when only using the first and the second component.

$\tau_{3}$ was fitted applying equation 8.1. At an emission wavelength of $592 \mathrm{~nm}, \tau_{3,0}$ is $9.7 \pm 1.0 \mathrm{~ms}$ and the calculated $\mathrm{E}_{A}$ is $108 \pm 44 \mathrm{meV}$ (Fig. 8.4(a)). This value is within the experimental uncertainty as calculated for the $1 \%$ doped sample $\left(\mathrm{E}_{A}\right.$ is $\left.129 \pm 42 \mathrm{meV}\right)$. For the $\mathrm{NaMgF}_{3}$ nanoparticles, $\mathrm{E}_{A}$ was $104 \pm 36 \mathrm{meV}$ for the $1 \% \mathrm{Eu}$ doped samples and an activation energy $\mathrm{E}_{A}$ of $95 \pm 40 \mathrm{meV}$ was measured for the $5 \%$ sample when exciting the sample at $592 \mathrm{~nm}$. Williams et al. calculated for the unknown defect at $500 \mathrm{~nm}$ an $\mathrm{E}_{A}$ of $139 \pm 20 \mathrm{meV}$. This 
comparable with the calculated values in this chapter within the experimental uncertainty [10]. $\mathrm{E}_{A}$ is similar for the $\mathrm{NaMgF}_{3}: \mathrm{Eu}$ and $\mathrm{RbMgF}_{3}: \mathrm{Eu}$ nanoparticles. Therefore, the unknown defect has likely the same origin in all the investigated samples. In Fig. 8.5, the average PL lifetimes of $\mathrm{Eu}^{3+}$ (a) and the unknown defect (b) are plotted for different $\mathrm{Eu}$ concentrations.

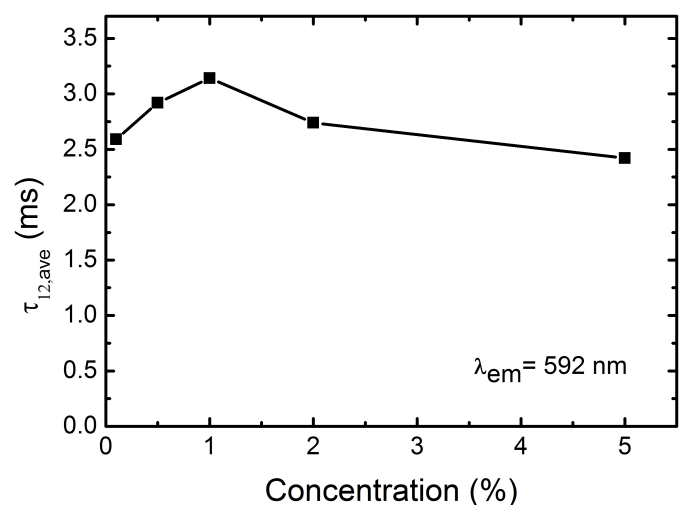

(a)

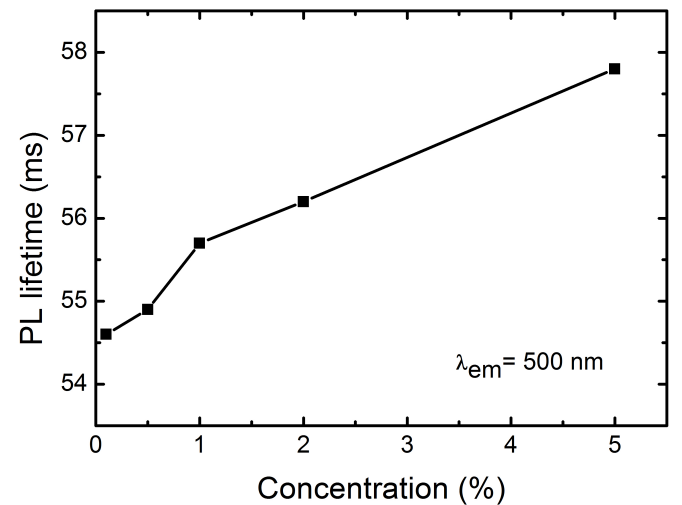

(b)

Figure 8.5: (a) Average PL lifetime for $\mathrm{Eu}^{3+}\left(\lambda_{e m}=592 \mathrm{~nm}, \lambda_{e x}=375 \mathrm{~nm}\right)$ doped $\mathrm{RbMgF}_{3}$ nanoparticles against the rare earth concentration and (b) for the broad unknown peak $\lambda_{e m}=500 \mathrm{~nm}$ and $\lambda_{e x}=375 \mathrm{~nm}$. The measurements were done at room temperature.

The $\mathrm{Eu}^{2+}$ lifetimes were measured with an emission wavelength of $360 \mathrm{~nm}$ for the ${ }^{6} \mathrm{P}_{7 / 2}$ and ${ }^{6} \mathrm{P}_{7 / 2}$ transitions and with an emission wavelength of $380 \mathrm{~nm}$ for the $\mathrm{E}_{g}$ transition $\left(\lambda_{e x}=256 \mathrm{~nm}\right) . \mathrm{Eu}^{3+}$ was measured with an emission wavelength of $592 \mathrm{~nm}$ while excited at $375 \mathrm{~nm}$. For the $1 \%$ Eu doped samples, the PL lifetimes the Eu ions were as high as $2.3 \pm 0.2 \mathrm{~ms}$ at $360 \mathrm{~nm}, 0.97 \pm 0.10 \mathrm{~ms}$ at $380 \mathrm{~nm}$ and $3.14 \pm 0.31 \mathrm{~ms}$ at $592 \mathrm{~nm}$ [11]. 


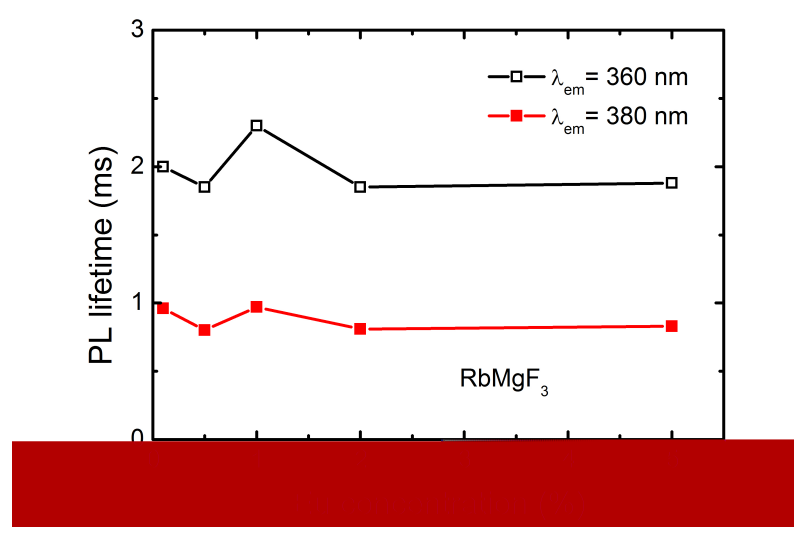

(a)

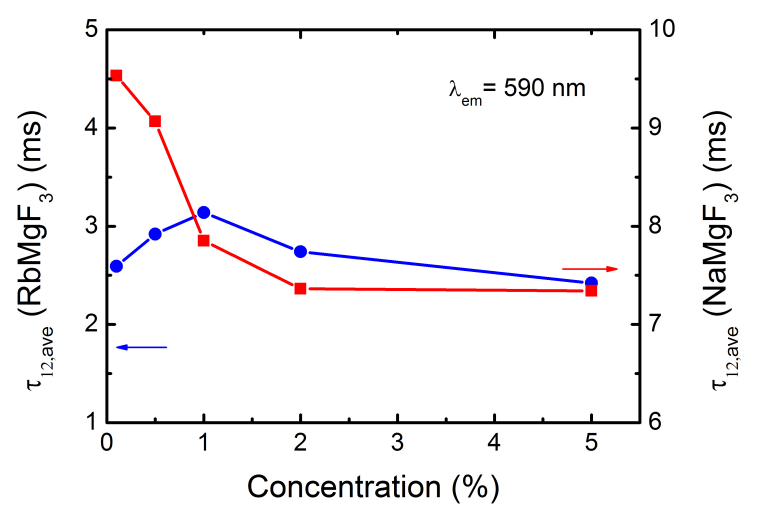

(c)

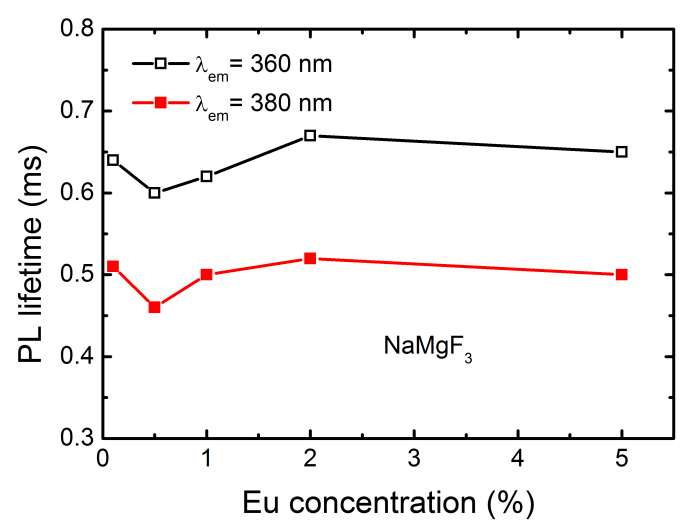

(b)

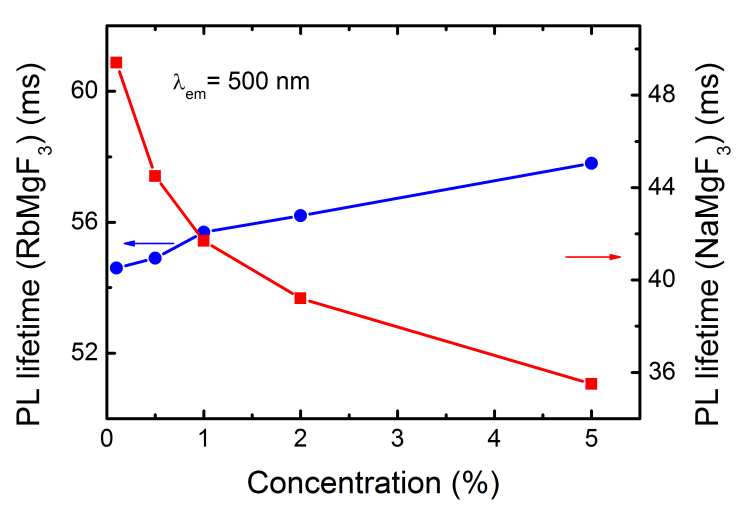

(d)

Figure 8.6: (a) Average PL lifetime for $\mathrm{Eu}^{2+}\left(\lambda_{e m}=360 \mathrm{~nm}\right.$ : open squares and $\lambda_{e m}=380$ $\mathrm{nm}$ : filled squares at $\left.\lambda_{e x}=256 \mathrm{~nm}\right)$ doped $\mathrm{RbMgF}_{3}$ nanoparticles against the rare earth concentration and

(b) average PL lifetime for $\mathrm{Eu}^{2+}\left(\lambda_{e m}=360 \mathrm{~nm}\right.$ : open squares and $\lambda_{e m}=380$ $\mathrm{nm}$ : filled squares at $\left.\lambda_{e x}=256 \mathrm{~nm}\right)$ doped $\mathrm{NaMgF}_{3}$ nanoparticles against the rare earth concentration.

(c) PL lifetime for the $\mathrm{Eu}^{3+}$ ion with $\lambda_{e m}=590 \mathrm{~nm}$ and $\lambda_{e x}=375 \mathrm{~nm}$ for the $\mathrm{RbMgF}_{3}$ (circles) and $\mathrm{NaMgF}_{3}$ (squares).

(d) PL lifetime for the broad peak at $\lambda_{e m}=500 \mathrm{~nm}$ and $\lambda_{e x}=375 \mathrm{~nm}$ for the $\mathrm{RbMgF}_{3}$ (circles) and $\mathrm{NaMgF}_{3}$ (squares).

The measurements were done at room temperature. 
The above Fig. 8.6 shows a summary of the average lifetimes for different ions in nanoparticles. Fig. 8.6 $(\mathrm{a}+\mathrm{b})$ show the lifetimes for the $\mathrm{Eu}^{2+}$ ion in the nanoparticles and it was observed the lifetimes are not dependent of the Eu concentration. The lifetimes for the $\mathrm{Eu}^{3+}$ are plotted in Fig. 8.6(c) and the PL lifetime increases until it reaches a maximum after a concentration $1 \% \mathrm{Eu}$. After this initial increase the PL lifetime starts to decrease until a concentration of $5 \% \mathrm{Eu}$. The average PL lifetime decreases with increasing $\mathrm{Eu}$ concentration for the $\mathrm{NaMgF}_{3}$ nanoparticles. For the $0.5 \%$ sample, the highest $\mathrm{I}_{{ }_{7}} / \mathrm{I}_{7} F_{1}$ ratio was calculated and it was as high as 1.9:1. This indicates, the higher this ratio the more distorted is the average crystal environment of the $\mathrm{Eu}^{3+}$ ion. Due to the production of the nanoparticles the crystal environment of the $0.5 \%$ sample could have changed and become more distorted. The PL lifetime of the broad defect centred around $470 \mathrm{~nm}$ increases for $\mathrm{RbMgF}_{3}$ and decreases for $\mathrm{NaMgF}_{3}$ with increasing Eu concentration. The radiative lifetimes and quantum efficiencies were calculated for the $\mathrm{Eu}^{3+}$ ions and are listed in Tab. 8.1

Table 8.1: Summary of the different measured PL lifetimes, $\tau_{12, \text { ave }}$, the radiative lifetime, $\tau_{\text {rad }}$, and the quantum efficiency, $\eta$, for $\mathrm{RbMgF}_{3}$ doped with different concentrations of $\mathrm{Eu}[11]$.

\begin{tabular}{|l|l|l|l|}
\hline Concentration (\%) & $\tau_{12, \text { ave }}(\mathrm{ms})$ & $\tau_{\text {rad }}(\mathrm{ms})$ & $\eta(\%)$ \\
\hline \hline \multirow{3}{*}{0.1} & $2.0 \pm 0.2(\mathrm{em} @ 360 \mathrm{~nm})$ & & \\
& $0.96 \pm 0.09(\mathrm{em} @ 380 \mathrm{~nm})$ & & \\
& $54.6 \pm 5.5(\mathrm{em} @ 500 \mathrm{~nm})$ & & \\
& $2.59 \pm 0.26(\mathrm{em} @ 592 \mathrm{~nm})$ & 6.73 & 38.5 \\
\hline \multirow{3}{*}{0.5} & $1.9 \pm 0.2(\mathrm{em} @ 360 \mathrm{~nm})$ & & \\
& $0.8 \pm 0.1(\mathrm{em} @ 380 \mathrm{~nm})$ & & \\
& $54.9 \pm 5.5(\mathrm{em} @ 500 \mathrm{~nm})$ & & \multirow{2}{*}{ @ 38.9} \\
\hline & $2.9 \pm 0.3(\mathrm{em} @ 592 \mathrm{~nm})$ & 5.97 & \\
\hline
\end{tabular}

Continued on next page 
Table 8.1 - Continued from previous page

\begin{tabular}{|c|c|c|c|}
\hline Concentration (\%) & $\tau_{12, \text { ave }}(\mathrm{ms})$ & $\tau_{\text {rad }}(\mathrm{ms})$ & $\eta(\%)$ \\
\hline 1 & $\begin{array}{l}0.97 \pm 0.1(\mathrm{em} @ 380 \mathrm{~nm}) \\
55.7 \pm 5.6(\mathrm{em} @ 500 \mathrm{~nm}) \\
3.1 \pm 0.3(\mathrm{em} @ 592 \mathrm{~nm})\end{array}$ & 7.47 & 42.0 \\
\hline 2 & $\begin{array}{l}1.9 \pm 0.2(\mathrm{em} @ 360 \mathrm{~nm}) \\
0.8 \pm 0.1(\mathrm{em} @ 380 \mathrm{~nm}) \\
56.2 \pm 5.6(\mathrm{em} @ 500 \mathrm{~nm}) \\
2.7 \pm 0.3(\mathrm{em} @ 592 \mathrm{~nm})\end{array}$ & 7.58 & 36.1 \\
\hline 5 & $\begin{array}{l}1.9 \pm 0.2(\mathrm{em} @ 360 \mathrm{~nm}) \\
0.8 \pm 0.1(\mathrm{em} @ 380 \mathrm{~nm}) \\
57.8 \pm 5.8(\mathrm{em} @ 500 \mathrm{~nm}) \\
2.4 \pm 0.2(\mathrm{em} @ 592 \mathrm{~nm})\end{array}$ & 6.23 & 38.9 \\
\hline
\end{tabular}

All calculated $\tau_{\text {rad }}$ are greater than the measured PL lifetimes and this indicates there are non-radiative decays in the crystal. $\tau_{12 \text {,ave }}$ slightly decreases with increasing $\mathrm{Eu}^{3+}$ concentration (for the $1 \% \mathrm{Eu}$ till $5 \% \mathrm{Eu}$ doped samples). This was also observed for the $\mathrm{NaMgF}_{3}: \mathrm{Eu}$ nanoparticles, see chapter 6. But the lifetimes of the $\mathrm{Eu}^{3+}$ sites are lower than observed for the $\mathrm{NaMgF}_{3}$ nanoparticles. Therefore, it can be assumed that the energy transfer with increasing concentration is already saturated. This is also illustrated in Fig. 8.7(b) where the PL lifetime decay curves are plotted for the different Eu concentrations. 


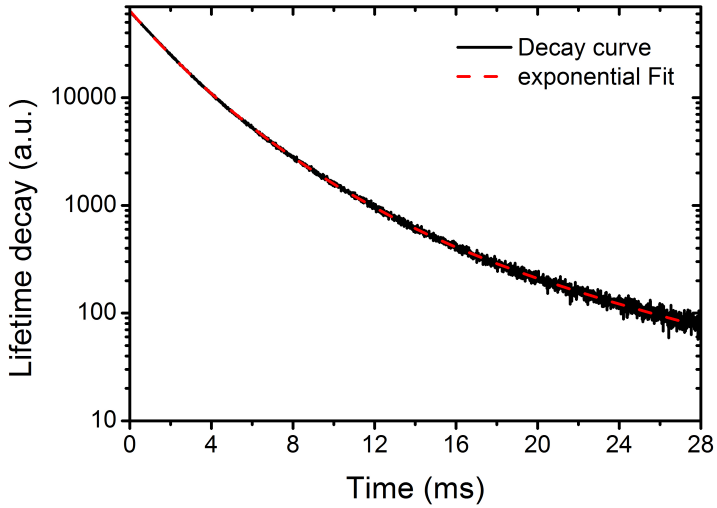

(a)

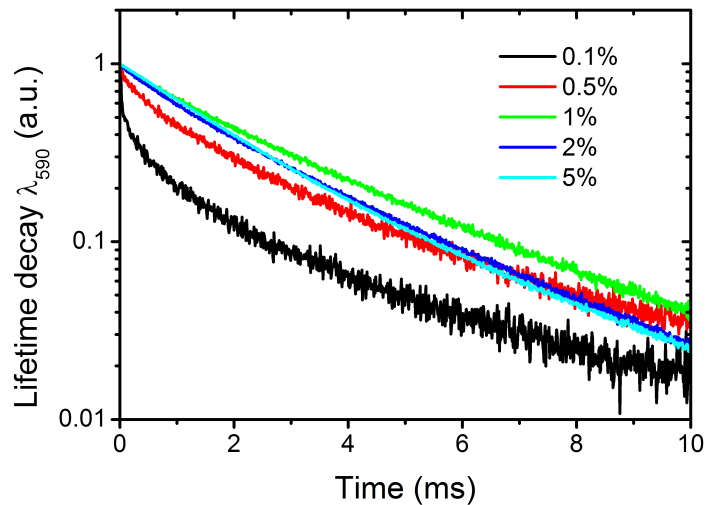

(b)

Figure 8.7: (a) PL lifetime decay of $5 \% \mathrm{Eu}$ doped $\mathrm{RbMgF}_{3}$ at $\lambda_{\text {em }}=590 \mathrm{~nm}$ (solid curve). The dashed curve is the exponential fit. The lifetime was measured at $\lambda_{e x}=$ $375 \mathrm{~nm}$.

(b) The lifetime decay curves at $\lambda_{e m}=590 \mathrm{~nm}$ emission for the different $\mathrm{Eu}$ concentrations in $\mathrm{NaMgF}_{3}$.

$\tau_{\text {rad }}$ can also be used to calculate the quantum efficiency. The quantum efficiency for the $0.1 \%$ doped sample was similar to the $5 \%$ Eu sample. The quantum efficiency was as high as $48.9 \%$ for the $0.5 \%$ doped nanoparticles. The lifetime of the unknown defect was measured with an emission wavelength of $500 \mathrm{~nm}\left(\lambda_{e x}=375 \mathrm{~nm}\right)$. The average lifetimes are listed in Tab. 8.1. It was observed that the lifetime increases with increasing Eu concentration. The average PL lifetime was as high as $57.8 \pm 5.8 \mathrm{~ms}$ for the $5 \%$ doped sample. The lifetimes of the $\mathrm{RbMgF}_{3}$ and $\mathrm{NaMgF}_{3}$ bulk materials are listed in Tab. 8.2. For the $\mathrm{Eu}^{2+}$ and $\mathrm{Eu}^{3+}$ ions in $\mathrm{NaMgF}_{3}$ the PL lifetimes of the bulks and nanoparticles are comparable. An exception is the lifetime of the unknown defect centred at $470 \mathrm{~nm}$. This PL lifetime is about $10 \%$ higher for the bulk materials compared to the nanoparticles which might be due to the distribution of the traps in the samples. The nanoparticles show a shorter lifetime for the unknown defect. This might be due to the existence of more defects next to it. This makes this transition more likely which results in a shorter lifetime. 
For the different $\mathrm{RbMgF}_{3}: \mathrm{Eu}$ samples (nanoparticles and bulk materials), the calculated lifetime with an emission wavelength of $360 \mathrm{~nm}$ for the $\mathrm{Eu}^{2+}$ ion are similar. The lifetime of the transition from the $5 \mathrm{~d}$ level is higher for the nanoparticles. This might be caused that the bulks were measured with an emission wavelength at $400 \mathrm{~nm}$ and the nanoparticles at $380 \mathrm{~nm}$. This difference in the emission wavelength could cause a selection of a different inequivalent site of $\mathrm{Eu}^{2+}$. The lifetime of the unknown defect is also higher in the $\mathrm{RbMgF}_{3}$ bulk materials, which might be due to the distribution of the traps in the samples.

Table 8.2: Summary of the measured PL lifetimes, $\tau_{\text {ave }}$, for different Eu-ions and the broad peak around $470 \mathrm{~nm}$ for $\mathrm{RbMgF}_{3}$ and $\mathrm{NaMgF}_{3}$ bulk materials.

\begin{tabular}{|l|l|l|}
\hline Compound & $\lambda_{\text {em }}(\mathrm{nm})$ & $\tau_{\text {ave }}(\mathrm{ms})$ \\
\hline \hline $\mathrm{NaMgF}_{3}$ & 360 & $0.63 \pm 0.06$ \\
& 390 & $0.42 \pm 0.04$ \\
& 500 & $67 \pm 7$ \\
& 592 & $11.1 \pm 1.1$ \\
\hline & 360 & $2.0 \pm 0.2$ \\
$\mathrm{RbMgF}_{3}$ & 400 & $0.51 \pm 0.05$ \\
& 500 & $68 \pm 7$ \\
\hline
\end{tabular}

In Fig. 8.8, the quantum efficiencies for the $\mathrm{RbMgF}_{3}$ and $\mathrm{NaMgF}_{3}$ nanoparticles are plotted versus the $\mathrm{Eu}$ concentration. The overall quantum efficiencies for the $\mathrm{RbMgF}_{3}$ samples are lower than the quantum efficiencies calculated for the $\mathrm{NaMgF}_{3}$ nanoparticles. Both of the quantum efficiencies follow the same behaviour. The highest $\eta$ was calculated for the 0.5 $\%$ samples which also shows the highest concentration of distorted defects near the $\mathrm{Eu}^{3+}$ sites. 


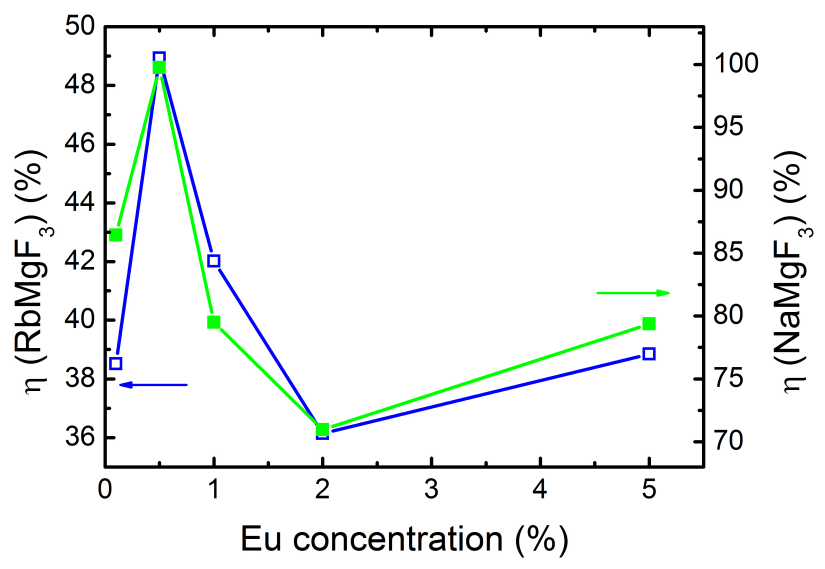

Figure 8.8: Quantum efficiency, $\eta$, for $\mathrm{RbMgF}_{3}$ and $\mathrm{NaMgF}_{3}$ against the Eu concentration.

The Judd-Ofelt parameters, $\Omega_{2}$ and $\Omega_{4}$, for the $\mathrm{Eu}^{3+}$ ions in $\mathrm{RbMgF}_{3}$ nanoparticles were calculated [65, 66, 92]. The parameters were estimated from equation 3.4, where $\mathrm{I}_{0-J}$ are the values from the PL spectra in Fig. 8.2 at room temperature after subtracting the unknown defect observed at $\sim 470 \mathrm{~nm}$. The resulting $\Omega_{2}$ and $\Omega_{4}$ values are listed in Tab. 8.3. $\Omega_{2}$ and $\Omega_{4}$ are $8.81 \times 10^{-20} \mathrm{~cm}^{2}$ and $32.7 \times 10^{-20} \mathrm{~cm}^{2}$ for the $1 \%$ doped Eu sample and $13.4 \times 10^{-20} \mathrm{~cm}^{2}$ and $33.4 \times 10^{-20} \mathrm{~cm}^{2}$ for $5 \% \mathrm{Eu}$, respectively. For the $\mathrm{NaMgF}_{3}$ sample the calculated values of $\Omega_{2}$ and $\Omega_{4}$ were $6.87 \times 10^{-20} \mathrm{~cm}^{2}$ and $25 \times 10^{-20} \mathrm{~cm}^{2}$ for $1 \% \mathrm{Eu}$ and $7.52 \times 10^{-20} \mathrm{~cm}^{2}$ and $27.9 \times 10^{-20} \mathrm{~cm}^{2}$ for $5 \%$ Eu doped $\mathrm{NaMgF}_{3}$ (see Tab. 6.2). As mentioned for the $\mathrm{NaMgF}_{3}$ :Eu nanoparticles, $\Omega_{2}$ is found in ranges of $\sim 1 \times 10^{-20} \mathrm{~cm}^{2}$ to $33 \times 10^{-20} \mathrm{~cm}^{2}$ in other nanoparticle compounds, and the higher $\Omega_{2}$ is the more $\mathrm{Eu}^{3+}$ is in a distorted site [22, 93, 94, 95]. $\Omega_{4}$ is found in ranges from $(0.2-13) \times 10^{-20} \mathrm{~cm}^{2}$ [93]. It is not clear why $\Omega_{4}$ is higher for the $\mathrm{RbMgF}_{3}$ : $\mathrm{Eu}^{3+}$ as found in other compounds. 
Table 8.3: The Judd-Ofelt parameters $\Omega_{2}$ and $\Omega_{4}$ for ${ }^{5} \mathrm{D}_{0} \rightarrow{ }^{7} \mathrm{~F}_{2}$ transitions for different concentrations of Eu doped $\mathrm{RbMgF}_{3}$ nanoparticles measured using the PL emission.

\begin{tabular}{|l|l|l|}
\hline Concentration $(\%)$ & $\Omega_{2}\left(10^{-20} \mathrm{~cm}^{2}\right)$ & $\Omega_{4}\left(10^{-20} \mathrm{~cm}^{2}\right)$ \\
\hline \hline 0.1 & $9.3 \pm 0.9$ & $40 \pm 4$ \\
\hline 0.5 & $16.6 \pm 1.7$ & $27.5 \pm 2.8$ \\
\hline 1 & $8.8 \pm 0.9$ & $32.7 \pm 3.3$ \\
\hline 2 & $9.2 \pm 0.9$ & $29.4 \pm 2.9$ \\
\hline 5 & $13.4 \pm 1.3$ & $33.4 \pm 3.3$ \\
\hline
\end{tabular}

Fig. 8.9 shows the Judd-Ofelt parameters, $\Omega_{2}$ and $\Omega_{4}$, for the $\mathrm{Eu}^{3+}$ ions for $\mathrm{RbMgF}_{3}$ and $\mathrm{NaMgF}_{3} . \Omega_{2}$ has a comparable behaviour for the $\mathrm{NaMgF}_{3}$ and $\mathrm{RbMgF}_{3}$ nanoparticles. $\Omega_{2}$ of the $\mathrm{RbMgF}_{3}$ samples shows a maximum for the $0.5 \%$ Eu sample. This sample has also the highest quantum efficiency, $\eta$, and the average crystal environment of the $\mathrm{Eu}^{3+}$ is more distorted compared to the other samples of $\mathrm{RbMgF}_{3}$ with different Eu concentrations. $\Omega_{4}$ does not show a dependence of the Eu concentration or the crystal environment.

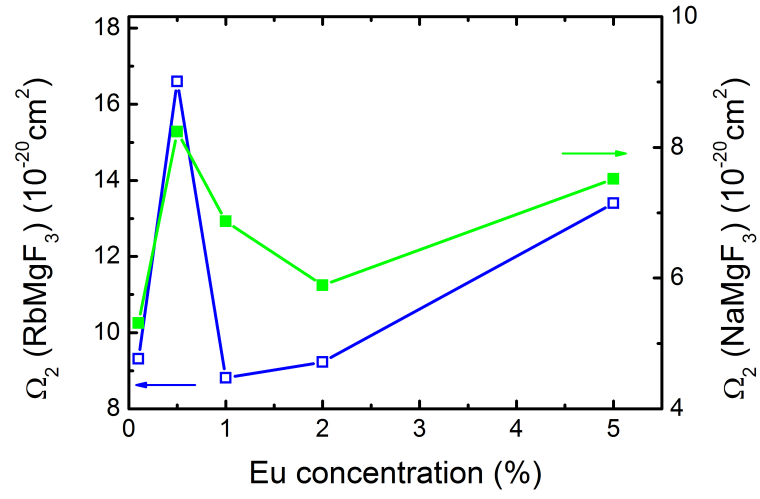

(a)

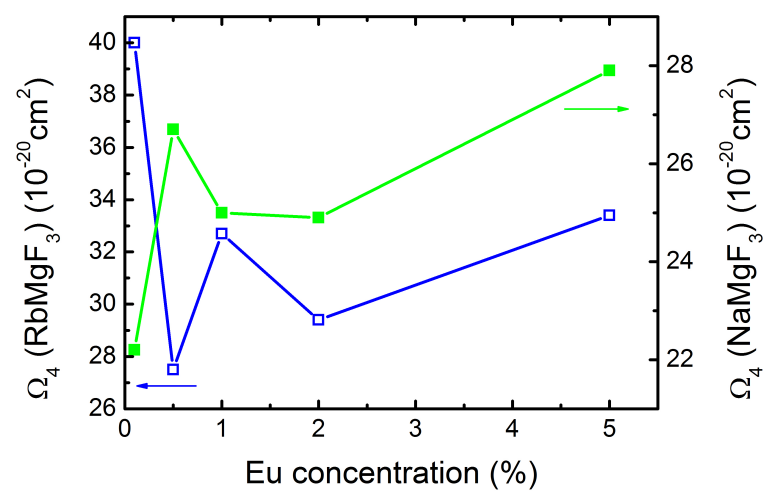

(b)

Figure 8.9: The Judd-Ofelt parameters $\Omega_{2}$ and $\Omega_{4}$ for the $\mathrm{Eu}^{3+}$ ions for $\mathrm{RbMgF}_{3}$ (a) and $\mathrm{NaMgF}_{3}$ (b) against the Eu concentration. 


\subsubsection{Mn doped $\mathrm{RbMgF}_{3}$ nanoparticles}

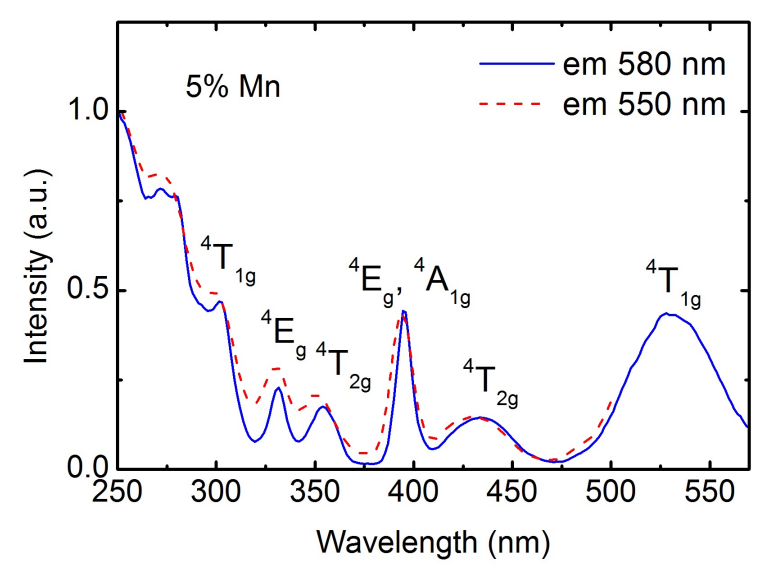

(a)

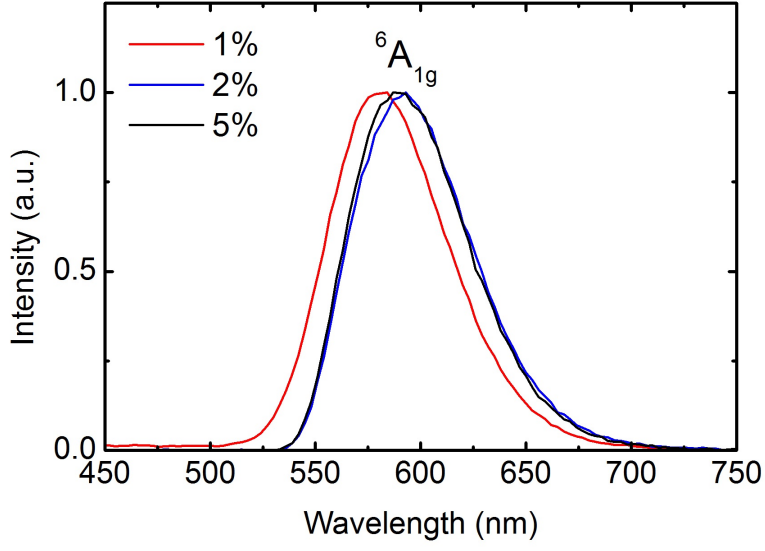

(b)

Figure 8.10: (a) PL excitation spectra for $5 \% \mathrm{Mn}$ doped $\mathrm{RbMgF}_{3}$ at $\lambda_{e m}=500 \mathrm{~nm}$ (dashed curve) and $\lambda_{e m}=600 \mathrm{~nm}$ (solid curve) for the distorted $\mathrm{Mn}^{2+}$ site [11].

(b) PL emission spectrum for $\mathrm{RbMgF}_{3}$ doped with $1 \% \mathrm{Mn}$ (red), $2 \%$ (blue) and $5 \% \mathrm{Mn}$ (black) excited at $396 \mathrm{~nm}$ [11].

PL excitation and emission spectra for the $\mathrm{RbMgF}_{3}: \mathrm{Mn}^{2+}$ nanoparticles are plotted in Fig. 8.10, where the PL excitation and emission peaks are consistent with $\mathrm{Mn}^{2+} \cdot \mathrm{Mn}^{2+}$ substitutes for $\mathrm{Mg}$, and $\mathrm{RbMgF}_{3}$ has the $\mathrm{P}_{6_{3} / m m c}$ space group with two inequivalent $\mathrm{Mg}$ sites. Therefore, two $\mathrm{Mn}^{2+}$ sites where expected: one site, $\mathrm{Mn} 1$, has $\mathrm{C}_{3 v}$ symmetry and the other site, $\mathrm{Mn} 2$, has $\mathrm{D}_{3 d}$ symmetry [3]. This difference in the crystal field between the two sites is large enough to be detected in the PL excitation spectra (Fig. 8.10(a)), resulting in a shift in the PL excitation peaks, when detecting at $550 \mathrm{~nm}$ or $580 \mathrm{~nm}$. The excited state levels of $\mathrm{Mn}^{2+}$ are indicated in Fig. 8.10(a) [11, 96, 97]. Fig. 8.10(b) shows the emission spectra of $1 \%$ (red), $2 \%$ (blue), and $5 \%$ (black) $\mathrm{Mn}^{2+}$ doped $\mathrm{RbMgF}_{3}$ nanoparticles. The emission spectra, when excited at $396 \mathrm{~nm}$, contains one peak at $580 \mathrm{~nm}$ for $1 \% \mathrm{Mn}$, and at $590 \mathrm{~nm}$ for $2 \%$ and $5 \% \mathrm{Mn}$. The small shift in the emission maximum to higher energies for the $1 \% \mathrm{Mn}^{2+}$ concentration might be due to a small change in the $\mathrm{Mn} 1 / \mathrm{Mn} 2$ 
ratio, in the different distribution of traps or in the crystal field [11, 96, 97]. The transition wavelength of the ${ }^{6} \mathrm{~A}_{1 g}$ level was found at $580 \mathrm{~nm}$ for the $\mathrm{RbMgF}_{3}: 0.2 \% \mathrm{Mn}$ doped bulk materials.

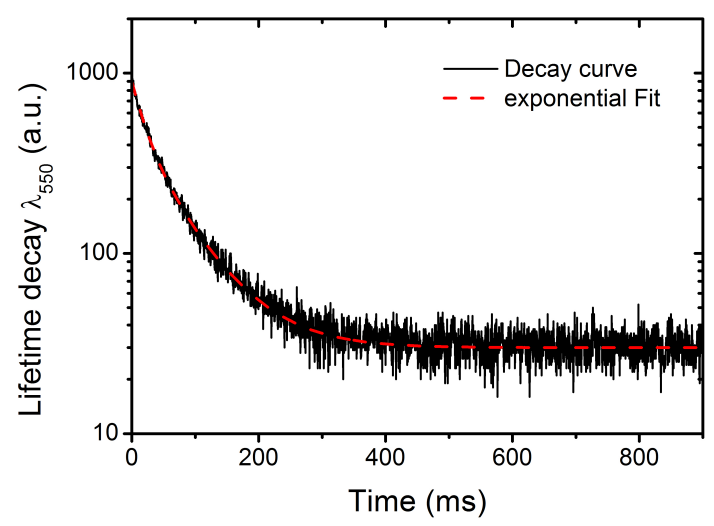

(a)

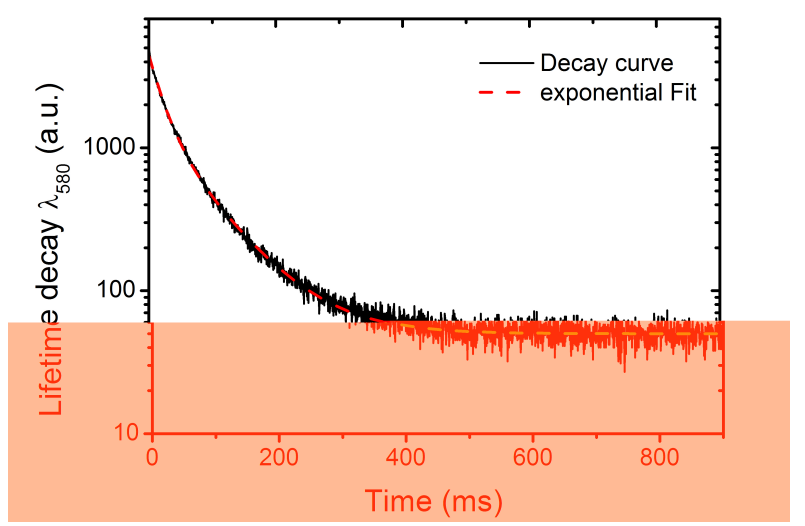

(b)

Figure 8.11: (a) PL lifetime decay of $5 \% \mathrm{Mn}$ doped $\mathrm{RbMgF}_{3}$ at $\lambda_{e m}=550 \mathrm{~nm}$ (solid curve) and (b) at $\lambda_{e m}=580 \mathrm{~nm}$ (solid curve). The dashed curve is the exponential fit. The lifetime was measured at $\lambda_{e x}=465 \mathrm{~nm}$.

PL lifetime measurements were performed with the Jobin-Yvon FluoroLog ${ }^{\circledR}$. The PL decay curves are illustrated in Fig. 8.11 for the $5 \%$ Mn doped sample for an emission wavelength at (a) $550 \mathrm{~nm}$ (solid curve) and for an wavelength (b) at $580 \mathrm{~nm}$ (solid curve). The lifetimes for different concentrations of Mn are summarised in Tab. 8.4. The PL decay could be fitted with two - exponential components and the average PL lifetimes, $\tau_{\text {ave }}$, were obtained from these components. PL lifetimes were measured at emission wavelengths of $550 \mathrm{~nm}$ and $580 \mathrm{~nm}$ for $\mathrm{RbMgF}_{3}$ nanoparticles for the two inequivalent $\mathrm{Mn}^{2+}$ sites. $\tau_{\text {ave }}$ slightly shifts to higher lifetimes for the $\mathrm{Mn}^{2+}$ site measured at an emission wavelength at $580 \mathrm{~nm}$ with increasing $\mathrm{Mn}^{2+}$ concentrations. The two calculated lifetimes measured for the $1 \% \mathrm{Mn}$ sample are in the same range within the uncertainty (Tab. 8.4). $\tau_{\text {ave }}$ is as large as $55 \pm 56 \mathrm{~ms}$ and comparable to the lifetimes observed in the $\mathrm{RbMgF}_{3}: \mathrm{Mn}$ bulk materials $(62.9 \pm 6.3 \mathrm{~ms})$ for the site observed at a emission wavelength at $580 \mathrm{~nm}$, see also 
chapter 7. The $\mathrm{RbMgF}_{3}$ bulk materials show a higher lifetime for the Mn site measured at an emission wavelength of $550 \mathrm{~nm}(87.7 \pm 9 \mathrm{~ms})$. The difference in the lifetimes of the two Mn sites in the nanoparticles is not as significant as it is calculated in the bulks and it remains unchanged with increasing $\mathrm{Mn}^{2+}$ concentration [11. The difference of the PL lifetimes of the $\mathrm{Mn}^{2+}$ in the nanoparticles compared to the bulk materials might be due to a higher concentration if distorted sites near $\mathrm{Mn}^{2+}$ in the nanoparticles resulting in a lower PL lifetime.

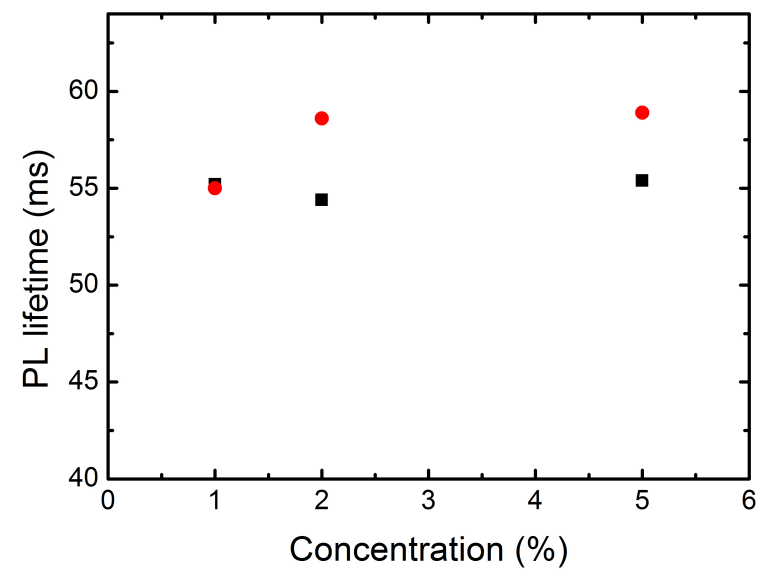

Figure 8.12: Average PL lifetime for the two inequivalent sites of $\mathrm{Mn}^{2+}\left(\lambda_{e m}=550 \mathrm{~nm}\right.$ circles and $\lambda_{e m}=580 \mathrm{~nm}$ - squares) in $\mathrm{RbMgF}_{3}$ nanoparticles against the rare earth concentration $\left(\lambda_{e x}=465 \mathrm{~nm}\right)$. 
Table 8.4: Summary of different measured PL lifetimes, $\tau_{\text {ave }}$, for $\mathrm{RbMgF}_{3}$ doped with different concentrations of Mn, exited at $465 \mathrm{~nm}$.

\begin{tabular}{|l|l|}
\hline Concentration $(\%)$ & $\tau_{\text {ave }}(\mathrm{ms})$ \\
\hline \hline & $55.0 \pm 5.5(\mathrm{em} @ 550 \mathrm{~nm})$ \\
& $55.2 \pm 5.5(\mathrm{em} @ 580 \mathrm{~nm})$ \\
\hline & $58.6 \pm 5.9(\mathrm{em} @ 550 \mathrm{~nm})$ \\
2 & $54.4 \pm 5.4(\mathrm{em} @ 580 \mathrm{~nm})$ \\
\hline & $58.9 \pm 5.9(\mathrm{em} @ 550 \mathrm{~nm})$ \\
5 & $55.4 \pm 5.5(\mathrm{em} @ 580 \mathrm{~nm})$ \\
\hline
\end{tabular}

\subsection{Thermoluminescence measurements}

Thermoluminescence measurements were performed on powdered samples immersed with isopropanol. All the measurements were performed after 20 minutes irradiation with an $\mathrm{X}$-ray generator operating at $40 \mathrm{~mA}$ and $40 \mathrm{kV}$. The data was collected using a PMT at a heating rate of $1 \mathrm{~K} / \mathrm{s}$, heating up from $300 \mathrm{~K}$ until $630 \mathrm{~K}$. The peaks were fitted with first order kinetics curve deconvolution.

\subsubsection{Eu doped $\mathrm{RbMgF}_{3}$ nanoparticles}

The TL measurements for the Eu doped nanoparticles are shown for the 1\%, $2 \%$ and $5 \%$ Eu doped samples. There was no TL detected for the $0.1 \%$ and $0.5 \%$ doped samples (Fig. ??). The data from the glow curves are summarised in Tab. 8.5. The temperature peaks of the glow curves for the different compounds of $\mathrm{RbMgF}_{3}$ are not comparable with each other. The normalised TL glow curves for the nanoparticles (1\% Eu doped $\mathrm{RbMgF}_{3}$ and $0.5 \% \mathrm{Eu}$ doped $\left.\mathrm{NaMgF}_{3}\right)$ and the bulk materials $\left(0.2 \% \mathrm{Eu}\right.$ doped $\left.\mathrm{RbMgF}_{3}\right)$ are plotted in Fig. 8.13(d). $\mathrm{RbMgF}_{3}$ bulk and nanoparticles seem have peaks at similar temperatures 
but the activation energies for the TL peaks in the nanoparticles are smaller than observed in the bulk materials (Tab. 7.6). These values for the $\mathrm{NaMgF}_{3}$ : Eu nanoparticles compare well with the values obtained for the $\mathrm{RbMgF}_{3}$ : Eu nanoparticles. These traps seem to arise from the same defects.

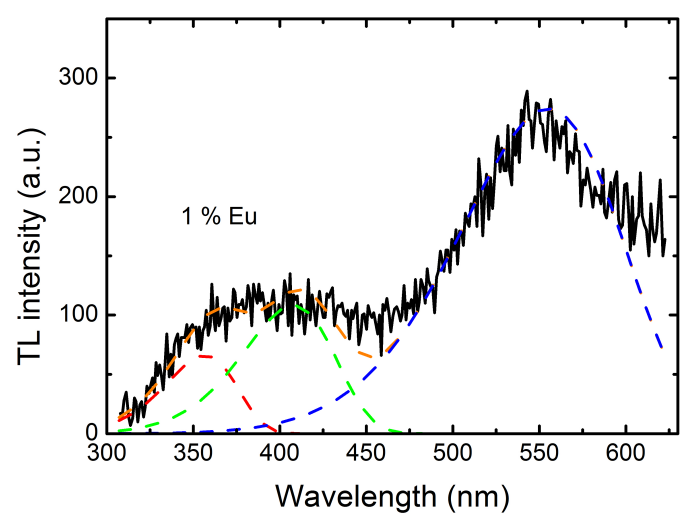

(a)

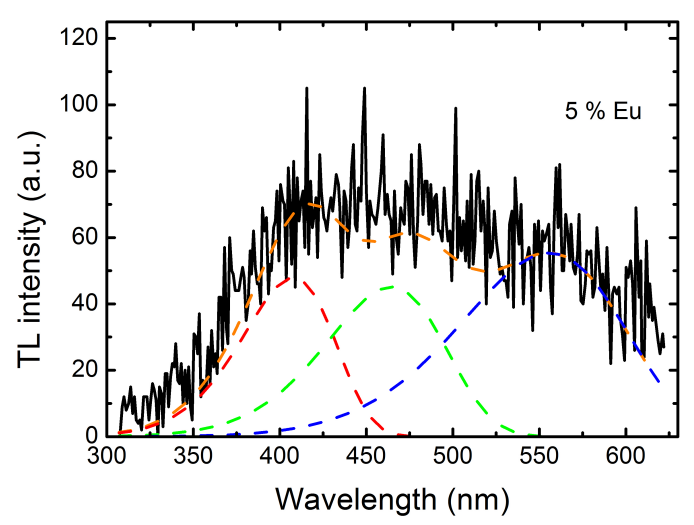

(c)

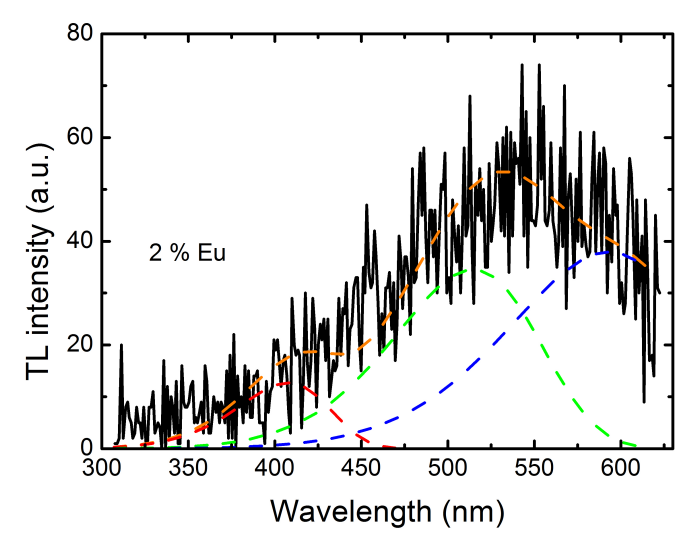

(b)

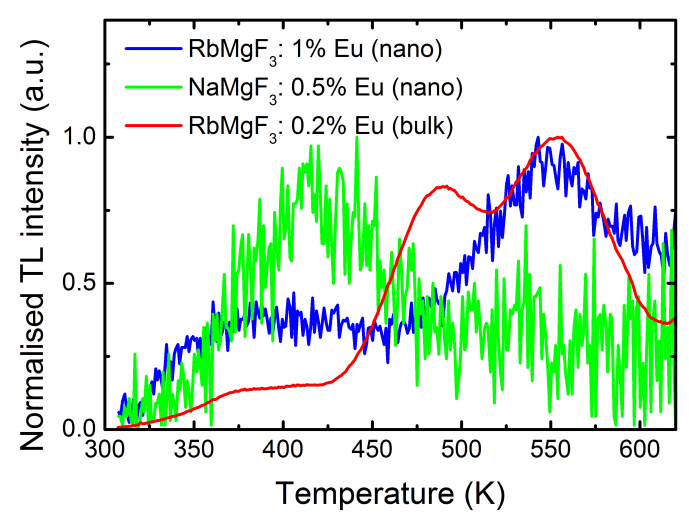

(d)

Figure 8.13: TL glow curves for $\mathrm{RbMgF}_{3}$ doped with $1 \%$ (a), $2 \%$ (b) and $5 \%$ (c) Eu after 20 min irradiation with the X-ray generator operating at $40 \mathrm{kV}$ and $40 \mathrm{~mA}$ (solid curves). The TL was detected with the PMT. The fitted peaks were obtained using first order kinetics glow curve deconvolution (dashed curves). (d) Normalised TL glow curves for $1 \%$ Eu doped $\mathrm{RbMgF}_{3}$ nanoparticles (blue curve), $0.5 \% \mathrm{Eu}$ doped $\mathrm{NaMgF}_{3}$ nanoparticles (green curve) and $0.2 \% \mathrm{Eu}$ doped $\mathrm{RbMgF}_{3}$ bulks (red curve). 
Table 8.5: The activation energies of the individual glow curve peaks for the $\operatorname{RbMgF}_{3}: 1 \%$, $2 \%$ and $5 \%$ Eu sample obtained by first order kinetics glow curve deconvolution. The top line is the activation energy for the glow curve.

\begin{tabular}{|l|l|l|l|}
\hline & $0.50 \pm 0.05 \mathrm{eV}$ & $0.50 \pm 0.05 \mathrm{eV}$ & $0.52 \pm 0.05 \mathrm{eV}$ \\
\hline \hline $1 \%$ & $357 \mathrm{~K}$ & $408 \mathrm{~K}$ & $555 \mathrm{~K}$ \\
peak height & 70 & 110 & 270 \\
\hline $2 \%$ & $408 \mathrm{~K}$ & $515 \mathrm{~K}$ & $593 \mathrm{~K}$ \\
peak height & 10 & 40 & 40 \\
\hline $5 \%$ & $408 \mathrm{~K}$ & $464 \mathrm{~K}$ & $556 \mathrm{~K}$ \\
peak height & 50 & 50 & 60 \\
\hline
\end{tabular}

\subsubsection{Mn doped $\mathrm{RbMgF}_{3}$ nanoparticles}

Fig. 8.14 shows the TL measurements on the Mn doped samples. The graph consist of low temperature peaks at $\sim 357 \mathrm{~K}$ for the $1 \%$, and $2 \% \mathrm{Mn}$ sample and at $\sim 359 \mathrm{~K}$ for the $5 \% \mathrm{Mn}$ sample. The activation energy for this low temperature peaks is $0.72 \mathrm{eV}$. The second TL peak with an activation energy of $0.58 \mathrm{eV}$ was measured at $375 \mathrm{~K}$ for the $1 \%$ doped sample and shifts to higher temperatures for the other Mn concentrations (379 K for the $2 \% \mathrm{Mn}$ and $378 \mathrm{~K}$ for the $5 \% \mathrm{Mn}$ sample). The activation energy of the peak centred at $\sim 415 \mathrm{~K}$ is $0.56 \mathrm{eV}$. The last two temperature peaks have an activation energy of $0.5 \mathrm{eV}$ and the peaks are centred at $459 \mathrm{~K}$ and $536 \mathrm{~K}$ for the $1 \%$ sample and at higher temperatures for the $2 \%$ sample ( $461 \mathrm{~K}$ and $539 \mathrm{~K}$ ) shifting to lower temperatures for the $5 \%$ sample $(454 \mathrm{~K}$ and $523 \mathrm{~K})$. Fig. $8.14(\mathrm{~d})$ shows the normalised TL glow curves for the nanoparticles ( $5 \% \mathrm{Mn}$ doped $\mathrm{RbMgF}_{3}$ and $5 \% \mathrm{Mn}$ doped $\mathrm{NaMgF}_{3}$ ) and the

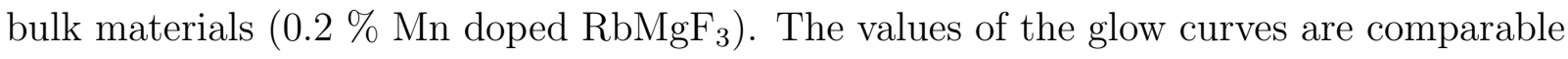
to each other. The low temperature TL peaks for $\mathrm{RbMgF}_{3}$ nanoparticles, at $\sim 346 \mathrm{~K}$ and $\sim 374 \mathrm{~K}$, were also observed for the bulk materials but the activation energies were different 
for the different compounds (see Tab. 7.4). However, the nanoparticles show mainly low temperature peaks.

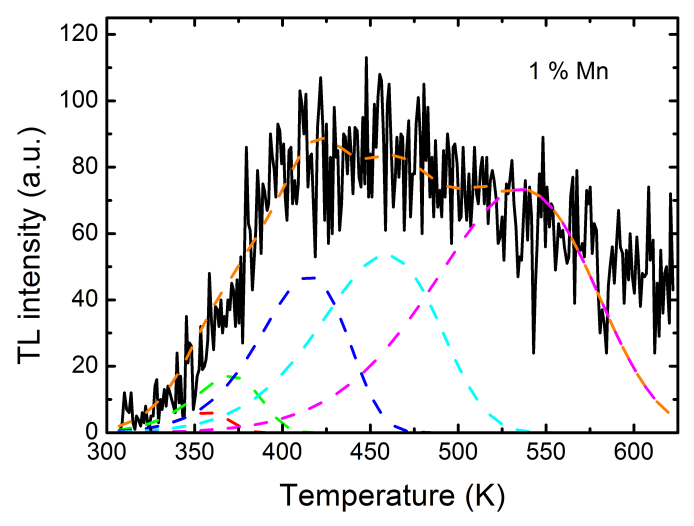

(a)

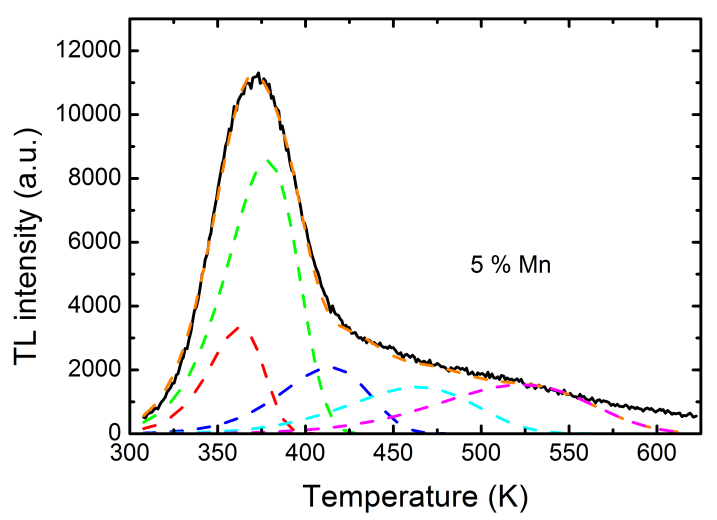

(c)

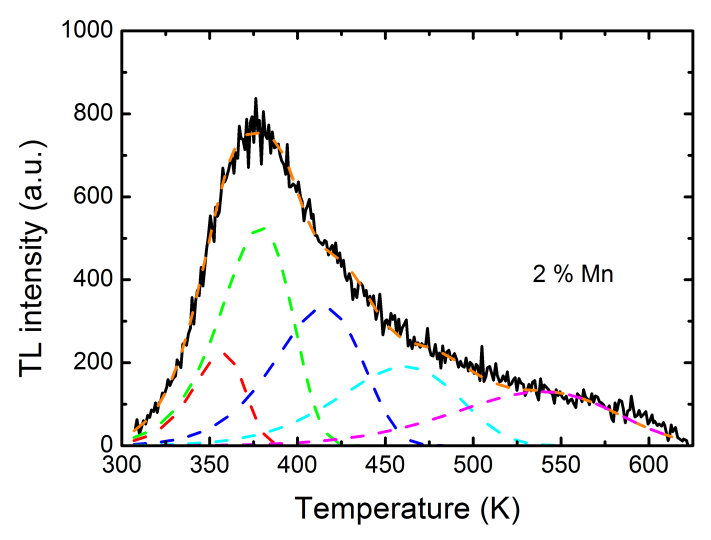

(b)

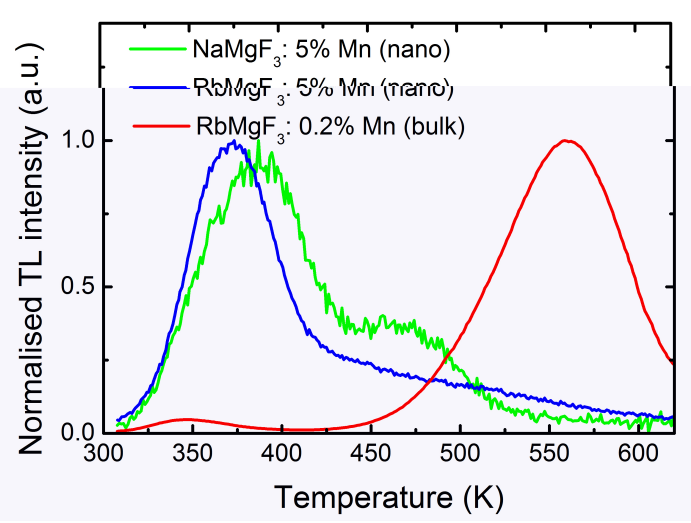

(d)

Figure 8.14: TL glow curves for $\mathrm{RbMgF}_{3}$ doped with $1 \% \mathrm{Mn}(\mathrm{a}), 2 \% \mathrm{Mn}$ (b), $5 \% \mathrm{Mn}$ (c) after 20 min irradiation with the X-ray generator operating at $40 \mathrm{kV}$ and 40 $\mathrm{mA}$ (solid curves). The TL was detected with the PMT. The fitted peaks were obtained using first order kinetics glow curve deconvolution (dashed curves). (d) Normalised TL glow curves for $5 \% \mathrm{Mn}$ doped $\mathrm{RbMgF}_{3}$ nanoparticles (blue curve), $5 \% \mathrm{Mn}$ doped $\mathrm{NaMgF}_{3}$ nanoparticles (green curve) and $0.2 \%$ Mn doped $\mathrm{RbMgF}_{3}$ bulks (red curve).

These values for the $\mathrm{NaMgF}_{3} \mathrm{Mn}$ and Eu nanoparticles compare well with the values 
obtained for the and $\mathrm{RbMgF}_{3}$ nanoparticles (see Fig. 6.11 and Tab. 6.5).

Table 8.6: The activation energies of the individual glow curve peaks for $\mathrm{RbMgF}_{3}$ doped with different concentration of Mn obtained by first order kinetics glow curve deconvolution. The top line is the activation energy for the different peaks for the glow curves.

\begin{tabular}{|l|l|l|l|l|l|}
\hline & $\begin{array}{l}0.72 \pm 0.07 \\
\mathrm{eV}\end{array}$ & $\begin{array}{l}0.58 \pm 0.06 \\
\mathrm{eV}\end{array}$ & $\begin{array}{l}0.56 \pm 0.05 \\
\mathrm{eV}\end{array}$ & $\begin{array}{l}0.50 \pm 0.05 \\
\mathrm{eV}\end{array}$ & $\begin{array}{l}0.50 \pm 0.05 \\
\mathrm{eV}\end{array}$ \\
\hline \hline $1 \%$ & $357 \mathrm{~K}$ & $375 \mathrm{~K}$ & $415 \mathrm{~K}$ & $45 \mathrm{~K}$ & $536 \mathrm{~K}$ \\
peak height & 10 & 20 & 50 & 50 & 70 \\
\hline $2 \%$ & $357 \mathrm{~K}$ & $379 \mathrm{~K}$ & $415 \mathrm{~K}$ & $461 \mathrm{~K}$ & $539 \mathrm{~K}$ \\
peak height & 220 & 530 & 330 & 190 & 130 \\
\hline $5 \%$ & $359 \mathrm{~K}$ & $378 \mathrm{~K}$ & $414 \mathrm{~K}$ & $454 \mathrm{~K}$ & $523 \mathrm{~K}$ \\
peak height & 330000 & 8600 & 2100 & 1500 & 1500 \\
\hline
\end{tabular}

\subsection{Radioluminescence and temperature dependent RL measurements}

Continuous radioluminescence measurements were performed on pressed pellets with an X-ray generator operating at $40 \mathrm{kV}$ and $40 \mathrm{~mA}$ and collected using a CCD detector. The $5 \%$ samples were additional irradiated with the ${ }^{241} \mathrm{Am}$ source to observe their low dose behaviour. During this irradiation the signal was collected using a PMT detector. The temperature dependent RL measurements were performed on pulverised samples immersed with isopropanol under the same conditions as the RL measurements. The temperature was changed in $10 \mathrm{~K}$ steps and 10 measurements with and integration time of $5 \mathrm{~s}$ were performed for each temperature step. After a certain temperature step, the sample was thermally bleached by heating it up until $350{ }^{\circ} \mathrm{C}$. 


\subsubsection{Radioluminescence measurements}

\subsubsection{Eu doped $\mathrm{RbMgF}_{3}$ nanoparticles}

The following Fig. 8.15-8.17 show the RL spectra and the dependence of the integrated RL intensity on the dose for the $\mathrm{RbMgF}_{3}$ doped with different concentrations of Eu. The RL spectra from the $\mathrm{Eu} \mathrm{RbMgF}_{3}$ doped samples show a strong $\mathrm{Eu}^{3+}$ component and a weaker $\mathrm{Eu}^{2+}$ component. The $\mathrm{Eu}^{2+}$ line emission centred at $360 \mathrm{~nm}$ arises from the $\mathrm{Eu}^{2+} 4 \mathrm{f} \rightarrow 4 \mathrm{f}$ transition and the broader peak centred at $450 \mathrm{~nm}$ is due to the unknown defect as observed in the PL spectra. The higher ratio of the $\mathrm{Eu}^{2+}$ component to the $\mathrm{Eu}^{3+}$ component for the $0.1 \%$ sample indicates that this sample has a higher $\mathrm{Eu}^{2+}$ fraction. The ratio of $\mathrm{Eu}^{3+} / \mathrm{Eu}^{2+}$ increases with increasing Eu concentration. At $1.8 \mathrm{~Gy}$, the ratio is $2: 1$ for the $0.1 \% \mathrm{Eu}$ doped sample (Fig. 8.16(a)) and 7:1 for the $5 \%$ doped sample (Fig. 8.17(a)). The ratio changes after heavy irradiation $(12.7 \mathrm{kGy})$ and it is 11:1 for $0.1 \%$ doped sample, $25: 1$ for $0.5 \%$ Eu doped sample, 5:1 for the $1 \%$ sample and 38:1 for the $5 \%$ sample. The difference in the ratio for the $1 \%$ doped sample could be due to the preparation process. The $\mathrm{Eu}^{3+}$ RL emission wavelengths appear at the same wavelengths as observed in the PL emission spectra. The ratio $I_{7} F_{2} / I_{7} F_{1}$ remains unchanged after irradiation. It was calculated with 1:1.6 for the $0.1 \%$ Eu sample and decreases to 1:1.3 for the $5 \%$ sample. This decrease may be caused by the crystal environment of the $\mathrm{Eu}^{3+}$ which gets more distorted with increasing Eu concentration. For the PL spectra, a ratio $I_{7} F_{2} / I_{7} F_{1}$ of $1.1: 1$ for the $0.1 \%$ sample, 1.9:1 for the $0.5 \%$ sample, $1: 1$ for the $1 \%$ Eu doped sample, 1.1:1 for the $2 \%$ doped sample and 1.6:1 for the $5 \%$ sample was calculated. In the PL spectra, the crystal environment of $\mathrm{Eu}^{3+}$ is more distorted for the $5 \%$ sample than the $0.1 \%$ sample but there was not a increase in the ratio for the $0.5 \%$ sample observed. This might be caused by the effect that the PL is exciting directly in the band. The carriers generated by RL are more mobile and theses carriers might not recombine with the highly distorted site in the $0.5 \%$ sample. 


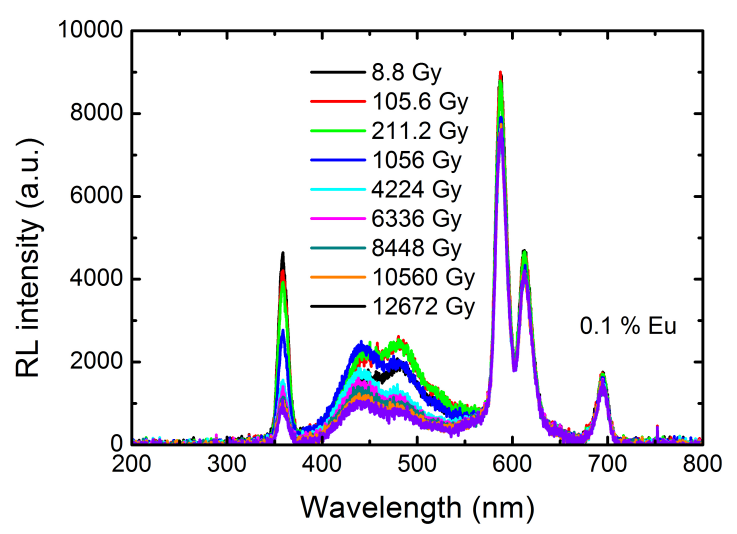

(a)

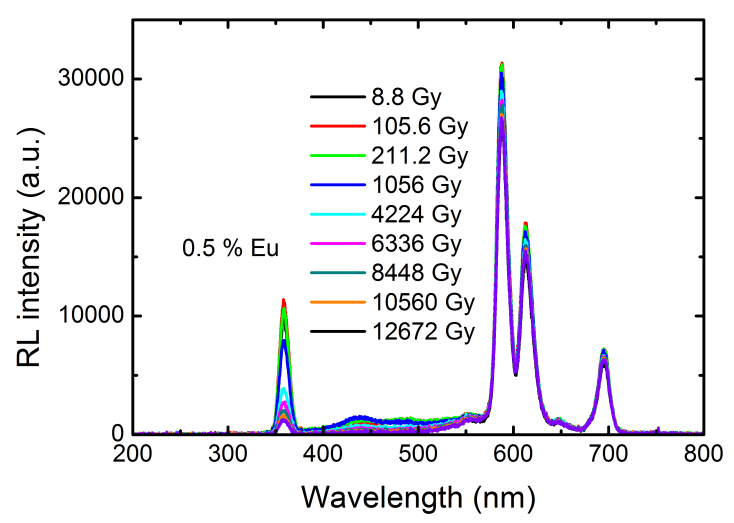

(c)

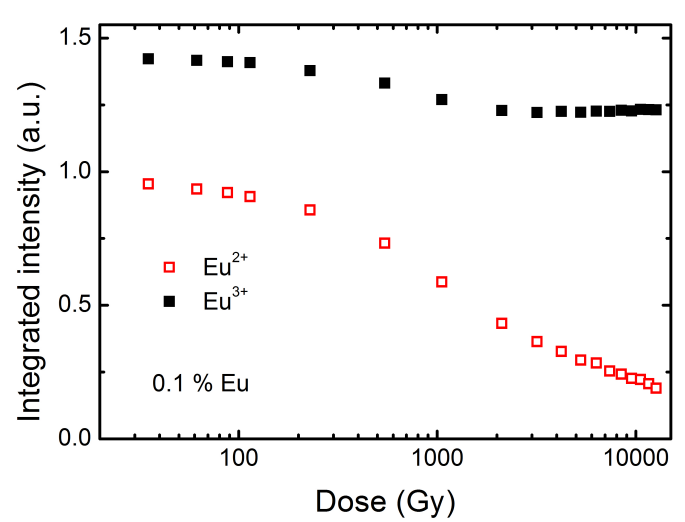

(b)

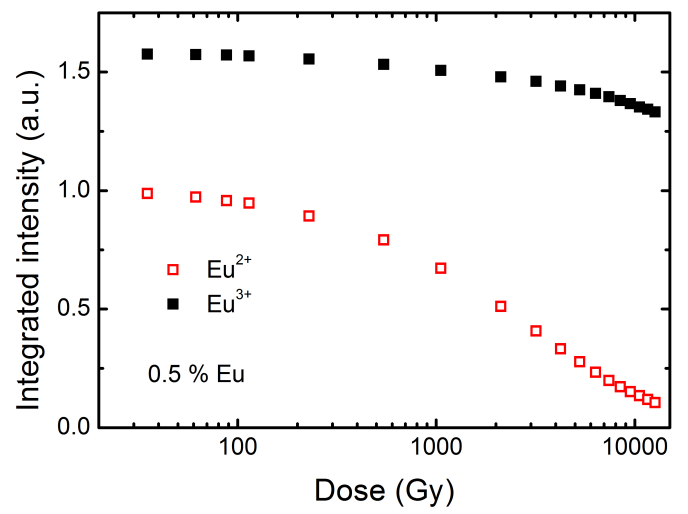

(d)

Figure 8.15: Radioluminescence spectra for $\mathrm{RbMgF}_{3}$ nanoparticles doped with $0.1 \% \mathrm{Eu}$ (a) and $0.5 \% \mathrm{Eu}(\mathrm{c})$ for different doses and the integrated RL spectra as a function of dose during continuous X-ray irradiation for $0.1 \% \mathrm{Eu}(\mathrm{b})$ and 0.5 $\% \mathrm{Eu}(\mathrm{d})$ doped samples ( $\mathrm{Eu}^{2+}$ open squares and $\mathrm{Eu}^{3+}$ filled squares).

All samples show a decrease in the RL signal after heavy irradiation. $\mathrm{I}_{R L}$ of the $0.1 \% \mathrm{Eu}$ sample decreases by $9.9 \%$ at $1 \mathrm{kGy}$ and by $12.7 \%$ at $12.7 \mathrm{kGy}$ for the $\mathrm{Eu}^{3+}$ ion. The RL signal for the $\mathrm{Eu}^{2+}$ ion decreases by $39.9 \%$ at $1 \mathrm{kGy}$ and by $80.6 \%$ at $12.7 \mathrm{kGy}$ (Fig. 8.15(b)). At $\sim 2 \mathrm{kGy}$, the RL intensity seems to be constant with increasing dose. The 0.5 $\%$ sample shows the highest decrease of the RL intensity. It decreases by $4.3 \%$ at $1 \mathrm{kGy}$ and by $15.5 \%$ at $12.7 \mathrm{kGy}$ for the $\mathrm{Eu}^{3+}$ ion. For the $\mathrm{Eu}^{2+}$ ion, the intensity decreases by 
$31.9 \%$ at $1 \mathrm{kGy}$ and by $89.3 \%$ at $12.7 \mathrm{kGy}$ (see Fig. 8.15(d)).

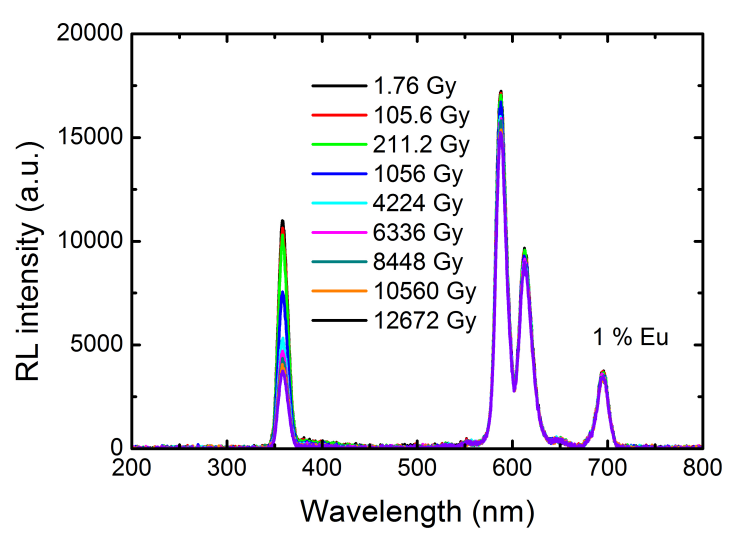

(a)

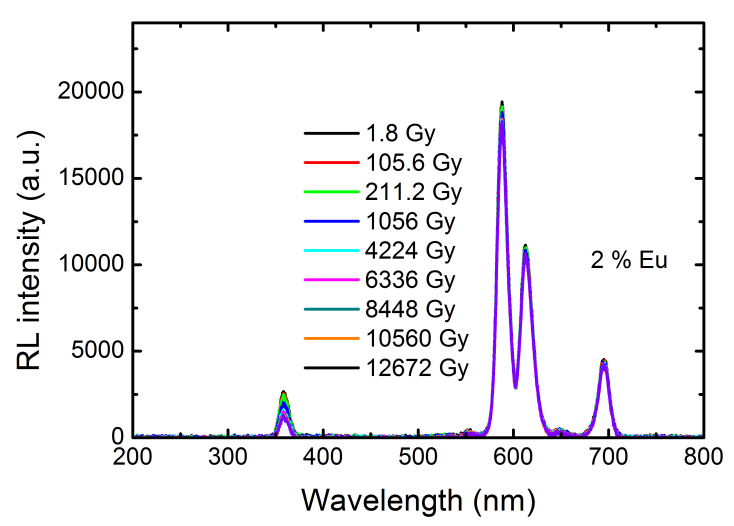

(c)

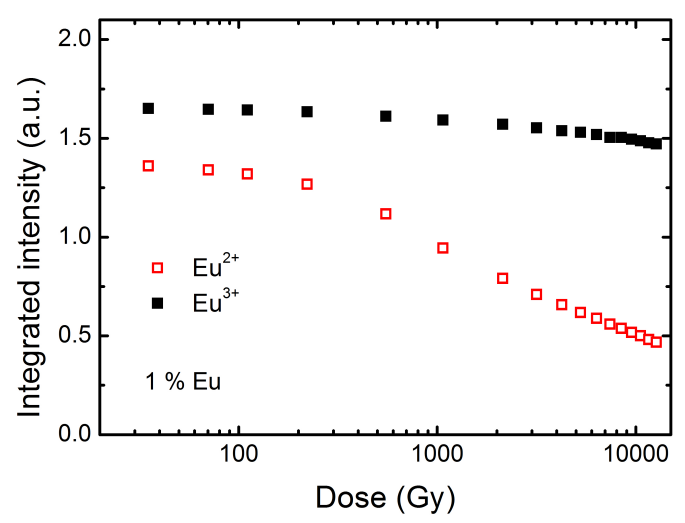

(b)

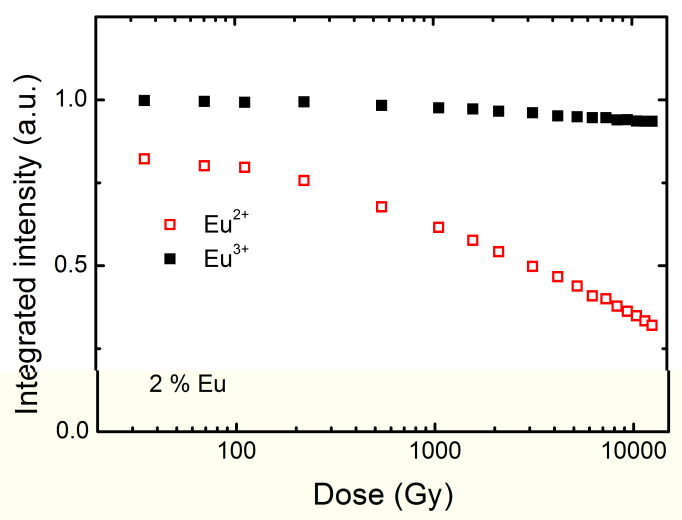

(d)

Figure 8.16: Radioluminescence spectra for $\mathrm{RbMgF}_{3}$ nanoparticles doped with $1 \% \mathrm{Eu}$ (a) and $2 \% \mathrm{Eu}(\mathrm{c})$ for different doses and the integrated RL spectra as a function of dose during continuous X-ray irradiation for $1 \% \mathrm{Eu}$ (c) and $2 \% \mathrm{Eu}$ (d) doped samples $\left(\mathrm{Eu}^{2+}\right.$ open squares and $\mathrm{Eu}^{3+}$ filled squares).

Fig. 8.16(a) shows the dose dependence of the RL signal for the $1 \%$ sample. The $\mathrm{Eu}^{3+}$ RL intensity decreases by $3.9 \%$ at $1 \mathrm{kGy}$ and $11.2 \%$ at $12.7 \mathrm{kGy}$. For the $\mathrm{Eu}^{2+}$ ion, the signal decreases by $31.7 \%$ at $1 \mathrm{kGy}$ and by $66.2 \%$ at $12.7 \mathrm{kGy}$. The lowest decrease of $\mathrm{I}_{R L}$ was calculated for the $2 \% \mathrm{Eu}$ doped sample where the signal of the $\mathrm{Eu}^{3+}$ ion decreases 
by $2.5 \%$ at $1 \mathrm{kGy}$ and by $6.7 \%$ at $12.7 \mathrm{kGy}$. For the $\mathrm{Eu}^{2+}$ ion it decreases by $25.1 \%$ at 1 kGy and by $61.0 \%$ at 12.7 kGy (Fig. 8.16(d)).

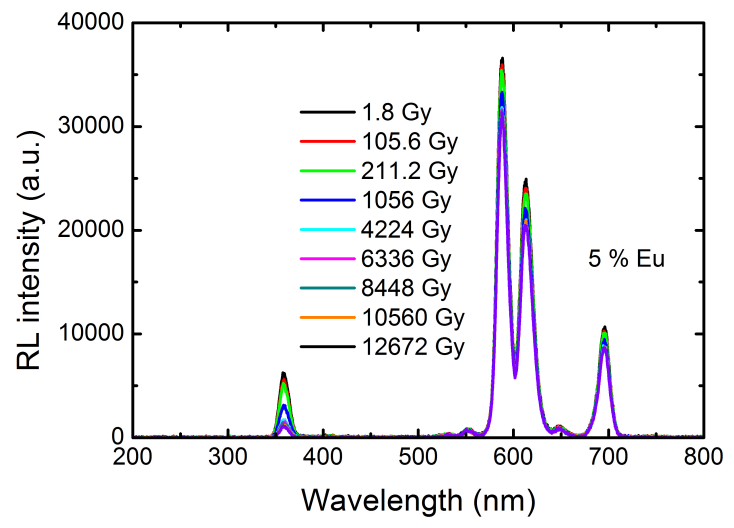

(a)

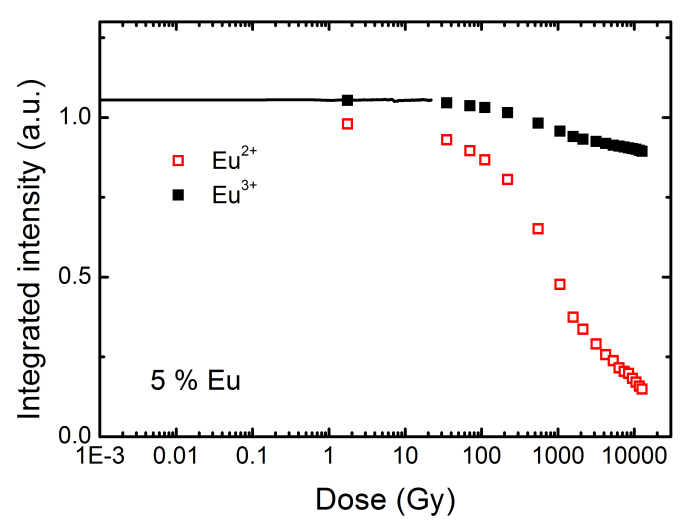

(b)

Figure 8.17: Radioluminescence spectra for $\mathrm{RbMgF}_{3}$ nanoparticles doped with $5 \% \mathrm{Eu}$ (a) for different doses and the integrated RL spectra as a function of dose during continuous X-ray irradiation for $5 \% \mathrm{Eu}$ (b) doped samples $\left(\mathrm{Eu}^{2+}\right.$ open squares and $\mathrm{Eu}^{3+}$ filled squares, solid line ${ }^{241} \mathrm{Am}$ irradiation). The $\gamma$-ray RL was scaled to match the X-ray RL.

In Fig. 8.17(b), the $\mathrm{I}_{R L}$ of the $5 \%$ doped sample is plotted. The RL intensity decreases by $9.2 \%$ at $1 \mathrm{kGy}$ and by $15.2 \%$ at $12.7 \mathrm{kGy}$ for the $\mathrm{Eu}^{3+}$ ion. For the $\mathrm{Eu}^{2+}$, the signal decreases by $51.3 \%$ at $1 \mathrm{kGy}$ and by $84.8 \%$ at $12.7 \mathrm{kGy}$. Fig. 8.17(b) shows the measurements with the ${ }^{241} \mathrm{Am}$ source which emits monochrome $\gamma$-rays (solid line). It shows that the RL intensity is dose independent down to at least 10 mGy. The decrease of the RL signal at high doses might be due to the generation of defects that act as non-radiative decay centres. $\mathrm{I}_{R L}$ of the $\mathrm{Eu}^{2+}$ decreases in a faster rate than the RL signal of $\mathrm{Eu}^{3+}$ ion. As already mentioned for the $\mathrm{NaMgF}_{3}$ nanoparticles, this might be caused by radiation induced defects that preferentially occur near the $\mathrm{Eu}^{2+}$ sites leading to more quenching of the $\mathrm{Eu}^{2+} \mathrm{RL}$ compared to the RL signal of the $\mathrm{Eu}^{3+}$ ion [11, 12]. The $0.1 \%$ sample (Fig. 8.15(a)) and $0.5 \%$ sample (Fig. 8.15(c)) show the unknown defect centred around 
$470 \mathrm{~nm}$ in their RL emission spectra. The intensity of the unknown defect decreases with increasing Eu concentration. For higher Eu concentration above $1 \%$, the unknown defect will not be detected (Fig. 8.16(a+b) and Fig. 8.17(a)). This unknown defect was also detected in the PL spectra for all the different Eu concentrations (Fig. 8.2) and the PL intensity of this defect decreases with increasing Eu concentration. The RL emission for the unknown defect in the $0.1 \%$ sample increases and reaches a maximum after a dose of 200 Gy. After this initial increase the RL intensity starts to decrease until $12.7 \mathrm{kGy}$ (Fig. 8.15(a)). The RL intensity is initially low because some of the X-ray generated electrons and holes become trapped and hence the electrons and holes available for RL is low. For a high enough dose, all of the traps are filled and hence all of the X-ray generated electrons and holes are available for RL. Thus, the RL will increase with dose until all of the electron and hole traps are filled. This explains the initial increase in the RL until 200 Gy. The RL starts to decrease at high doses due to X-ray induced radiation damage that results in non-radiative recombination sites. Thus, some of the X-ray generated electrons and holes are not available for RL due to non-radiative recombination, which leads to a reduction in the RL for high doses. This non- radiative centre competes with unknown defect and the probability for mobile carriers to recombine in this centre is higher compared to unknown defect.

\subsubsection{2 $\mathrm{Mn}$ doped $\mathrm{RbMgF}_{3}$ nanoparticles}

Fig. 8.18 and 8.19 show the RL emission spectra and dose dependence of the RL intensity of $\mathrm{RbMgF}_{3}$ with different concentrations of $\mathrm{Mn}$. The RL spectra can be completely attributed to the $\mathrm{Mn}^{2+}$ ion with RL emission centred at $580 \mathrm{~nm}$ for the $1 \%$ and $590 \mathrm{~nm}$ for the $2 \%$ and $5 \%$ Mn sample. The RL and PL emission wavelengths remain unchanged after heavy irradiation. In Fig. 8.18(b), the $\mathrm{I}_{R L}$ is plotted against the X-ray dose for $1 \% \mathrm{Mn}$ and the RL increases by $31.9 \%$ and reaches a maximum after a dose of $5.3 \mathrm{kGy}$. After this initial increase the RL intensity starts to decrease. After a dose of $12.7 \mathrm{kGy}$ it has been decreased by $3.8 \%$. This results with a total difference of the RL signal of $26.9 \%$. The $1 \% \mathrm{Mn}$ 
sample shows the biggest dependence for the RL signal on the dose. The RL intensity of the $2 \% \mathrm{Mn}$ doped sample increases by $8.3 \%$ and reaches a maximum after a dose of 3.2 kGy. After this initial increase the RL intensity starts to decrease. After a dose of 12.7 kGy it has been decreased by $8.9 \%$ (Fig. $8.18(\mathrm{~d})$ ). This results with a total difference of the RL signal of $1.5 \%$. The RL signal of the $2 \%$ Mn sample is less dose dependent compared to the other two samples.

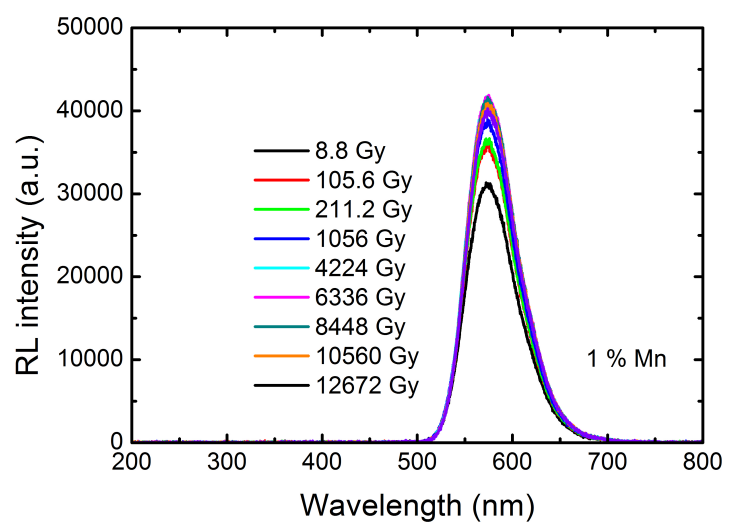

(a)

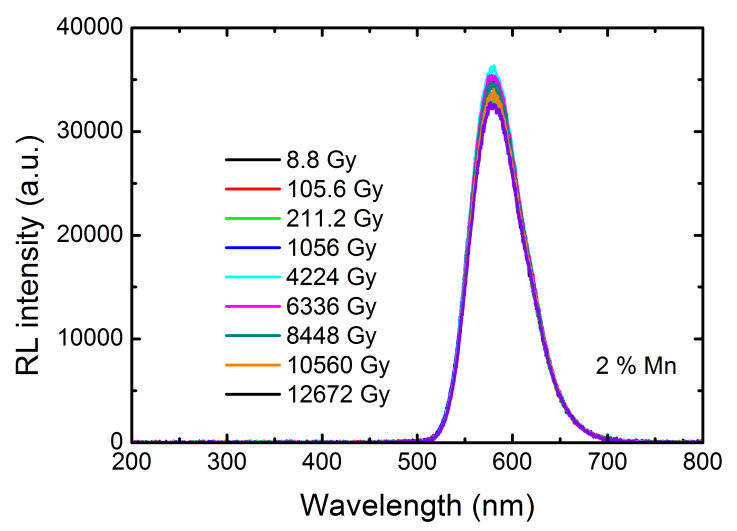

(c)

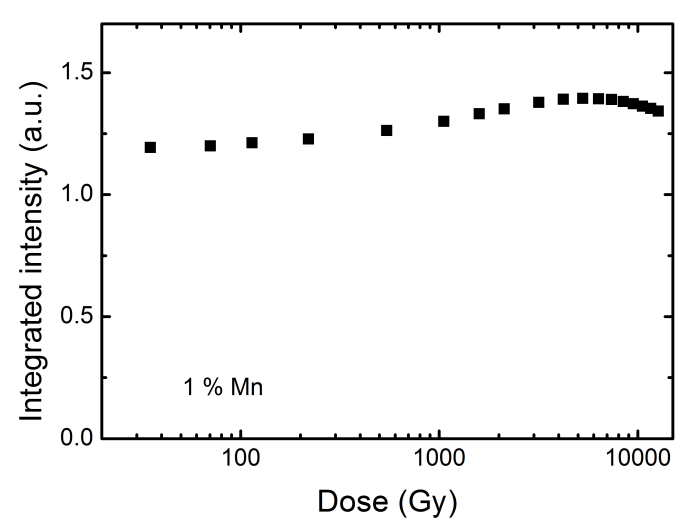

(b)

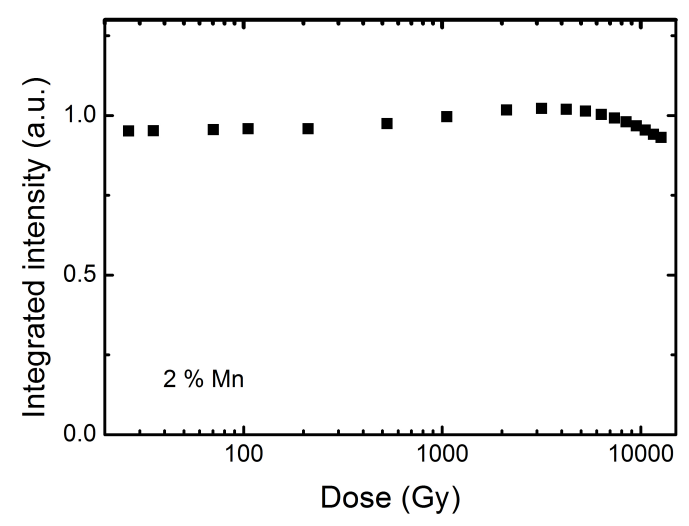

(d)

Figure 8.18: Radioluminescence spectra for $\mathrm{RbMgF}_{3}$ nanoparticles doped with $1 \% \mathrm{Mn}$ (a) and $2 \% \mathrm{Mn}$ (c) for different doses and the integrated RL spectra as a function of dose during continuous X-ray irradiation for $1 \% \mathrm{Mn}(\mathrm{b})$ and $2 \% \mathrm{Mn}$ (d) doped samples. 
For the $5 \%$ Mn doped sample, the RL intensity increases by $20.4 \%$ and reaches a maximum after a dose of $5.3 \mathrm{kGy}$. After this initial increase the RL intensity starts to decrease. After a dose of $12.7 \mathrm{kGy}$ it has been decreased by $5.3 \%$. This results with a total difference of the RL signal of $14.0 \%$ (Fig. 8.19(b)). Similar measurements of the RL intensity could not be performed for lower doses because the sensitivity of the CCD detector was not high enough. Therefore, additional low dose measurements were performed with the ${ }^{241} \mathrm{Am}$ source for the $5 \%$ doped sample. It was observed the RL signal is dose independent down to 10 mGy (solid line in Fig. 8.19(b)) [11].

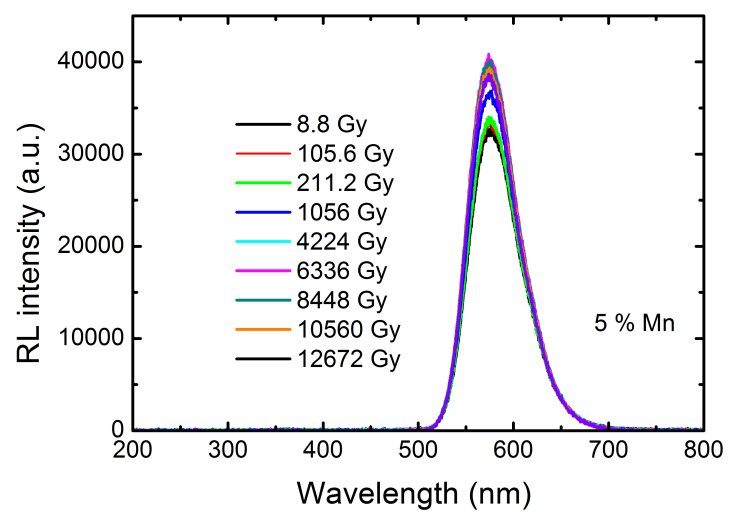

(a)

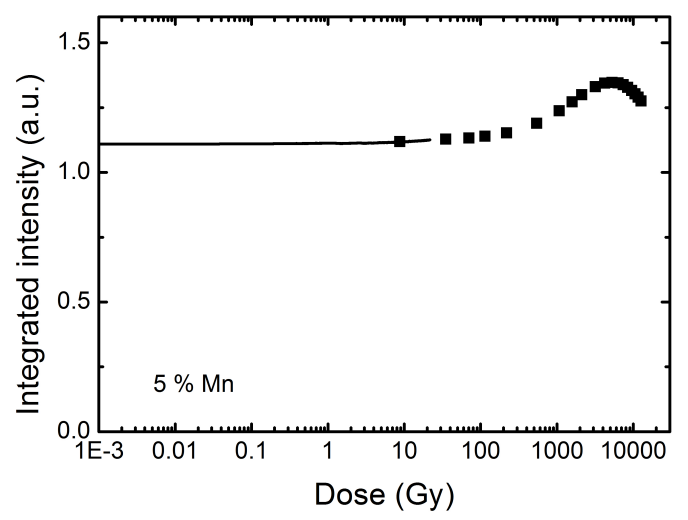

(b)

Figure 8.19: Radioluminescence spectra for $\mathrm{RbMgF}_{3}$ nanoparticles doped with $5 \% \mathrm{Mn}$ (a), for different doses and the integrated RL spectra as a function of dose during continuous X-ray irradiation for $5 \% \mathrm{Mn}$ (b) doped samples, solid line ${ }^{241} \mathrm{Am}$ irradiation. The $\gamma$-ray RL was scaled to match the X-ray RL.

For the $\mathrm{RbMgF}_{3}$ bulk materials, a similar behaviour of dose dependence of the RL signal was observed. The RL intensity for the bulk materials increases unitl it reaches a maximum after a dose of $\sim 1 \mathrm{kGy}$. After this initial increase the IRl intensity starts to decrease as observed for the nanoparticles (see Fig. 7.10(b)). The behaviour of the RL signal can be explained with the same model, which was used for the $\mathrm{RbMgF}_{3}$ : Eu doped bulk materials (see Fig. $7.8(\mathrm{c}+\mathrm{d})$ ). The RL intensity is initially low because some of the X-ray generated 
electrons and holes become trapped and hence electrons and holes available for RL are low. For a high enough dose, all of the traps are filled and hence all of the X-ray generated electrons and holes are available for RL. Thus, the RL will increase with dose until all of the electron and hole traps are filled. This explains the initial increase in the RL until $3.2 \mathrm{kGy}$ for the $2 \% \mathrm{Mn}$ and $5.3 \mathrm{kGy}$ for the $1 \%$ and $5 \% \mathrm{Mn}$ sample (Fig. 7.8(c)). The decrease of the RL signal for doses above $3.2 \mathrm{kGy}$ for the $2 \% \mathrm{Mn}$ and $5.3 \mathrm{kGy}$ for the $1 \%$ and $5 \%$ $\mathrm{Mn}$ is due to X-ray induced radiation damage that results in non-radiative recombination sites. Thus, as shown in Fig. 7.8(d), some of the X-ray generated electrons and holes are not available for RL due to non-radiative recombination, which leads to a reduction in the RL for high doses. Additional, there could be a conversion of $\mathrm{Mn}^{2+} \rightarrow \mathrm{Mn}^{3+}$. However, there was no $\mathrm{Mn}^{3+}$ in the PL spectra. This could be due to the PL of $\mathrm{Mn}^{3+}$ being too low to be detected. The nanoparticles show a similar behaviour of dose dependence in the RL compared to the bulk materials. However, the overall dose dependence of the RL is smaller for the nanoparticles. The RL intensity changed up to $104 \%$ for the $\mathrm{RbMgF}_{3}$ bulk materials and only up to $26.9 \%$ for the $1 \%$ doped nanoparticles. This leads to the conclusion that there is less generation of defects in the nanoparticles compared to the bulk materials. An initial increase of the RL signal is an indication that the nanoparticles should show OSL but no OSL was observed.

\subsubsection{Temperature dependent $\mathrm{RL}$ measurements}

\subsubsection{Eu doped $\mathrm{RbMgF}_{3}$ nanoparticles}

Fig. 8.20 and 8.21 show the temperature dependent RL measurements for the $0.1 \%, 1$ $\%$ and $5 \% \mathrm{RbMgF}_{3}$ nanoparticles. The integrated RL intensity of the $\mathrm{Eu}^{3+}$ ion increases by $0.9 \%$ compared to room temperature when the temperature is increased to $100{ }^{\circ} \mathrm{C}$ and decreases by $0.02 \%$ compared to $100{ }^{\circ} \mathrm{C}$ when the temperature is decreased to 40 ${ }^{\circ} \mathrm{C}$. The difference between the first value measured at $40{ }^{\circ} \mathrm{C}$ and the last one is $0.9 \%$ (Fig. 8.20(a) top graph). The RL signal of $\mathrm{Eu}^{2+}$ decreases by $31.8 \%$ compared to room 
temperature when the temperature is increased to $100{ }^{\circ} \mathrm{C}$ and the RL intensity decreases by $9.6 \%$ compared to $100{ }^{\circ} \mathrm{C}$ when the temperature is decreased to $40{ }^{\circ} \mathrm{C}$, resulting in a total difference for the RL intensity of $38.3 \%$ (Fig. 8.20(a) lower graph).

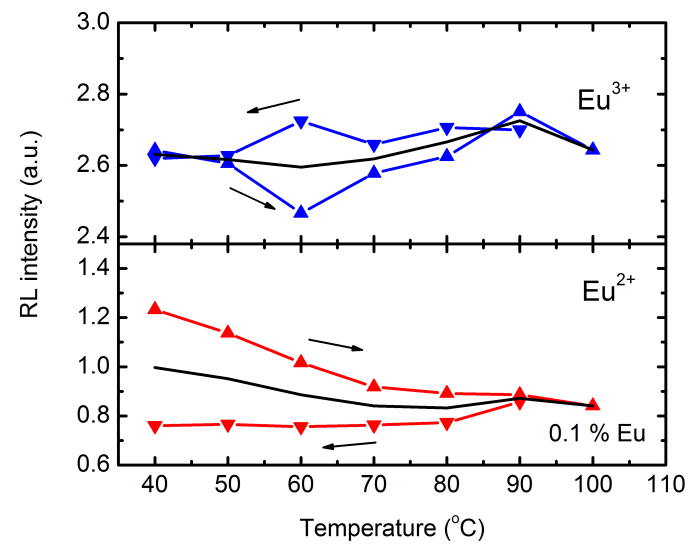

(a)

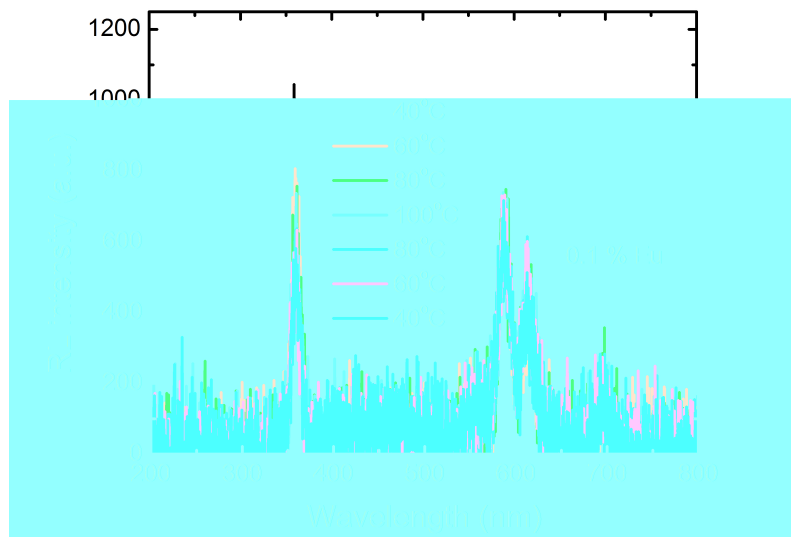

(b)

Figure 8.20: Temperature dependent RL spectra for $\mathrm{RbMgF}_{3}$ nanoparticles doped with 0.1 $\% \mathrm{Eu}(\mathrm{a})$ for different temperatures up to $100{ }^{\circ} \mathrm{C}$ and the respective RL spectra versus the wavelength for different temperatures ((b) for $0.1 \% \mathrm{Eu}) . \mathrm{Eu}^{2+}$ is indicated by the red colour and $\mathrm{Eu}^{3+}$ by the blue colour.

Arrows facing up signal an increase of temperature, and arrows facing down signal a decrease in temperature. Black curves show the trend of the temperature dependent RL.

The $\mathrm{Eu}^{3+}$ integrated intensity increases by $16.8 \%$ compared to room temperature when the temperature is increased to $100{ }^{\circ} \mathrm{C}$ and decreases by $15.9 \%$ compared to $100{ }^{\circ} \mathrm{C}$ when the temperature is decreased to $40{ }^{\circ} \mathrm{C}$, with a total decrease of the RL intensity by 2.1 $\%$ for the $1 \%$ Eu sample (Fig. 8.21(c)). $\mathrm{I}_{R L}$ of the $\mathrm{Eu}^{2+} \mathrm{RL}$ signal decreases by $14.5 \%$ compared to room temperature when the temperature is increased to $100{ }^{\circ} \mathrm{C}$ and decreases by $7.7 \%$ compared to $100{ }^{\circ} \mathrm{C}$ when the temperature is decreased to $40{ }^{\circ} \mathrm{C}$. A total decrease by $21.1 \%$ was calculated (see also Fig. 8.21(c)). The biggest change of the RL signal with temperature increasing was observed with the $5 \%$ Eu sample (Fig. 8.21(c)). The 
$\mathrm{RL}$ of $\mathrm{Eu}^{3+}$ increases by $101.7 \%$ compared to room temperature when the temperature is increased to $100{ }^{\circ} \mathrm{C}$ and decreases by $18 \%$ compared to $100{ }^{\circ} \mathrm{C}$ when the temperature is decreased to $40{ }^{\circ} \mathrm{C}$, resulting in a total difference of $65.3 \%$. The $\mathrm{Eu}^{2+}$ signal decreases by $14.2 \%$ at $100{ }^{\circ} \mathrm{C}$ and further decreases by $8.6 \%$ at $40{ }^{\circ} \mathrm{C}$, with a total difference of the RL signal of $21.6 \%$.

For the $\mathrm{Eu}^{2+}$ and $\mathrm{Eu}^{3+}$ ions, the change of the RL signal with the temperature shows a similar behaviour for all of the different concentrations of $\mathrm{Eu}$ in the nanoparticles. The $\mathrm{Eu}^{3+}$ signal increases with increasing temperature and the $\mathrm{Eu}^{2+}$ signal is slightly decreasing with increasing temperature. In section 5.3.1, the increase for the RL signal with increasing temperature was explained with a model, which explained the same effect in $\mathrm{NaMgF}_{3}$ : Eu bulk materials. (Fig. 5.16(d)). The model shows the RL signal depends on the normal RL trapping and the trapping of deep traps, $\tau_{d t}$. The probability of this trapping, $\tau_{d t}$, can be estimated from the following equation,

$$
\tau_{d t}=\tau_{o} e^{-\frac{\Delta_{1}}{k T}}
$$

where $\Delta_{1}$ is the energy of the level, $\mathrm{k}$ the Boltzmann's constant and $\mathrm{T}$ the temperature. It means, the probability, $\tau_{d t}$, of filling these deep traps increases with increasing temperature. Thus, the RL signal increases with increasing temperature, which was observed for the $\mathrm{Eu}^{3+}$ site in the $\mathrm{RbMgF}_{3}$ (Fig. 8.20 and 8.21). The decrease of the RL intensity with increasing temperature for the $\mathrm{Eu}^{2+}$ can be explained with an energy transfer to deep TL traps. These traps are filled during irradiation, but they show a non-radiative recombination. With temperature decreasing until $40{ }^{\circ} \mathrm{C}$, the RL intensity further decreases at a slower rate. The nanoparticles are irradiated for $5 \mathrm{~s}$ and ten measurements are performed at each temperature step. It was already mentioned in the previous chapter thermal bleaching is not enough to empty the deep traps. Therefore, the decrease in the RL intensity with decreasing temperature can be caused by the normal dose dependence of $\mathrm{I}_{R L}$. This was also observed for the $\mathrm{NaMgF}_{3}$ : Eu nanoparticles. 


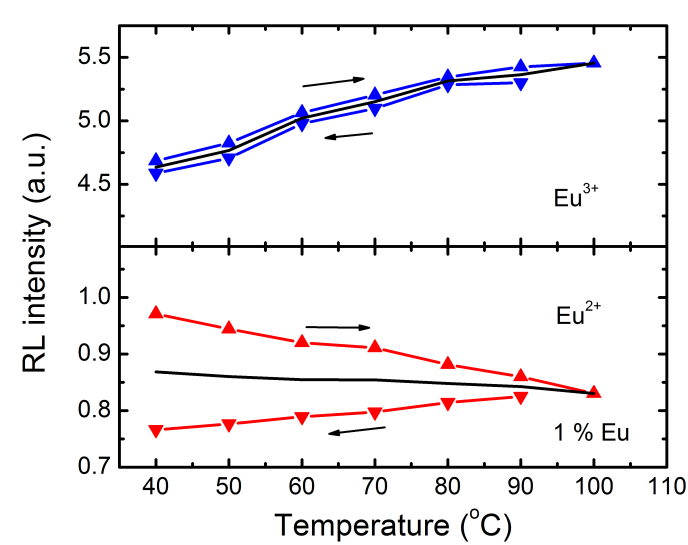

(a)

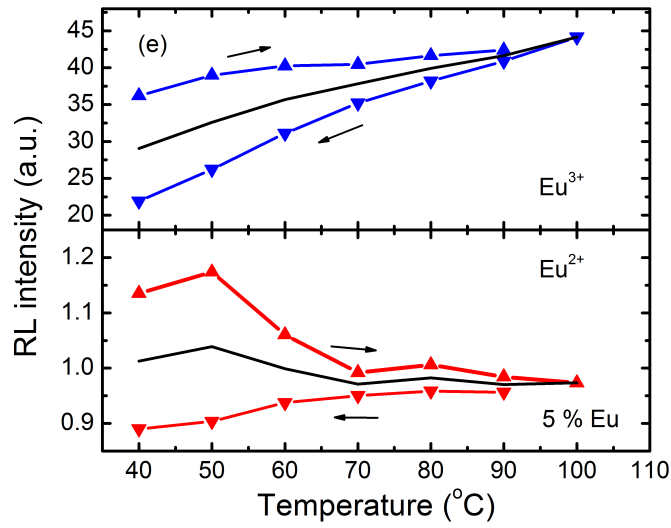

(c)

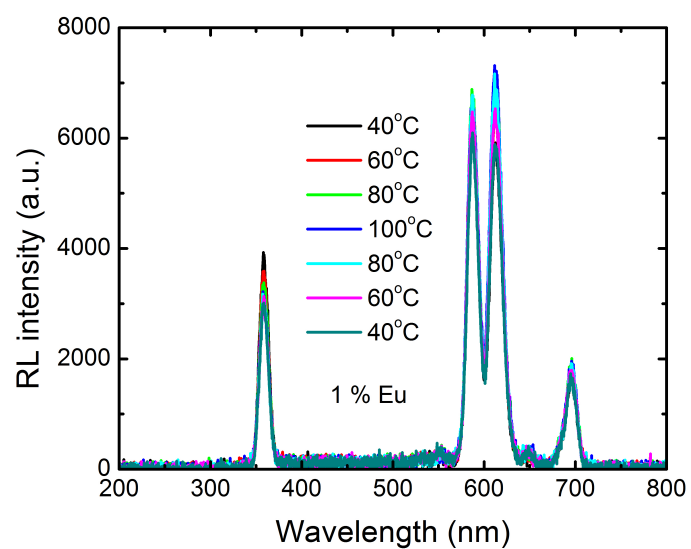

(b)

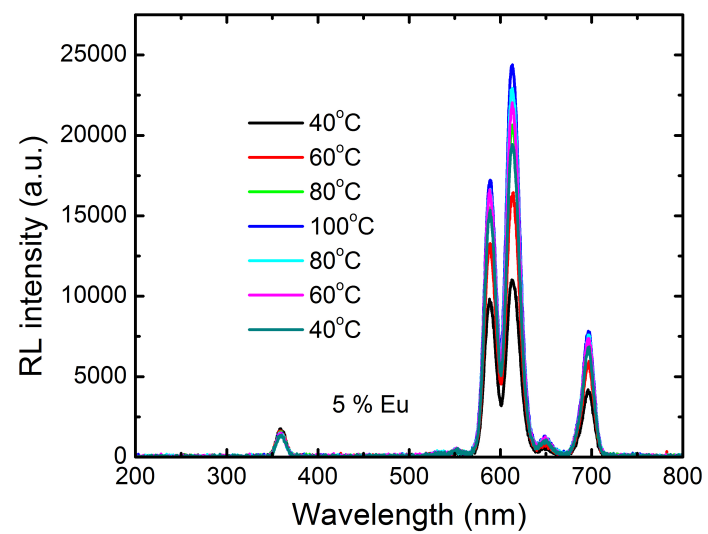

(d)

Figure 8.21: Temperature dependent RL spectra for $\mathrm{RbMgF}_{3}$ nanoparticles doped with 1 $\% \mathrm{Eu}(\mathrm{a})$ and $5 \% \mathrm{Eu}(\mathrm{c})$ for different temperatures up to $100{ }^{\circ} \mathrm{C}$ and the respective RL spectra versus the wavelength for different temperatures ((b) for $1 \% \mathrm{Eu}$ and (d) for $5 \% \mathrm{Eu}) . \mathrm{Eu}^{2+}$ is indicated by the red colour and $\mathrm{Eu}^{3+}$ by the blue colour.

Arrows facing up signal an increase of temperature, and arrows facing down signal a decrease in temperature. Black curves show the trend of the temperature dependent RL.

There was no observable shift in the RL emission wavelengths of $\mathrm{Eu}^{2+}$ and $\mathrm{Eu}^{3+}$ transitions with increasing temperature because the temperature change was too low. The ratio of 
$\mathrm{I}_{7} F_{2} / \mathrm{I}_{7} F_{1}$ changes with increasing temperature for the $5 \%$ sample. It is around 1.3:1 at $40{ }^{\circ} \mathrm{C}$ and it increases with increasing temperature to $1.5: 1$ at $100{ }^{\circ} \mathrm{C}$ and decreases again to $1.4: 1$ at $40{ }^{\circ} \mathrm{C}$. The increasing ratio $\mathrm{I}_{{ }^{F}} / \mathrm{I}_{7} F_{1}$ means the crystal environment of the $\mathrm{Eu}^{3+}$ gets more distorted when increasing the temperature. It decreases with decreasing temperature until it reaches the initial ratio of the first measurement at $40{ }^{\circ} \mathrm{C}$.

Tab. 8.7 summarises the thermal coefficient of the RL signal for different Eu-ions in the crystal. The thermal coefficient of the RL signal for the $\mathrm{Eu}^{2+}$ ion decreases with increasing temperature and is as high as $0.27 \pm 0.2 \% / \mathrm{K}$ for the $0.1 \% \mathrm{Eu}$ doped sample. It is as low as $0.07 \pm 0.1 \% / \mathrm{K}$ for the $5 \%$ sample. The opposite was observed for the $\mathrm{Eu}^{3+}$ ion. The thermal coefficient of the RL signal increases with increasing concentration of Eu. It is as low as $0.01 \pm 0.1 \% / \mathrm{K}$ for the $0.1 \%$ sample and as high as $0.24 \pm 0.05 \% / \mathrm{K}$ for $1 \%$ $\mathrm{Eu}$ in the nanoparticles. The thermal coefficient could not be calculated for the $5 \% \mathrm{Eu}$ doped sample because the oscillations in the measured RL intensity was too large. The $\mathrm{RbMgF}_{3}$ bulk materials showed a thermal coefficient of the RL of $0.13 \pm 0.1 \% / \mathrm{K}$ for the $0.2 \% \mathrm{Eu}^{2+}$ samples, which is comparable to the value as was calculated for the $0.1 \% \mathrm{Eu}$ doped nanoparticles.

Table 8.7: Summary of thermal coefficients of the RL signal for different doped samples and the $\mathrm{Eu}^{2+}$ and $\mathrm{Eu}^{3+}$ ions in $\mathrm{RbMgF}_{3}$ nanoparticles.

\begin{tabular}{|l|l|l|}
\hline Concentration $(\%)$ & $\mathrm{Eu}^{2+}(\% / \mathrm{K})$ & $\mathrm{Eu}^{3+}(\% / \mathrm{K})$ \\
\hline \hline 0.1 & $0.27 \pm 0.2$ & $0.01 \pm 0.1$ \\
\hline 1 & $0.08 \pm 0.1$ & $0.24 \pm 0.05$ \\
\hline 5 & $0.07 \pm 0.1$ & \\
\hline
\end{tabular}

\subsubsection{2 $\mathrm{Mn}$ doped $\mathrm{RbMgF}_{3}$ nanoparticles}

Fig. 8.22 shows the temperature dependence of the RL signal for the $1 \% \mathrm{Mn}(\mathrm{a}+\mathrm{b})$ and 5 $\% \mathrm{Mn}(\mathrm{c}+\mathrm{d})$ doped nanoparticles. The RL signal for the $1 \% \mathrm{Mn}$ doped sample increases 
by $10.7 \%$ compared to room temperature when the temperature is increased to $100{ }^{\circ} \mathrm{C}$ and increases by $6.9 \%$ compared to $100{ }^{\circ} \mathrm{C}$ when the temperature is decreased to $40{ }^{\circ} \mathrm{C}$, leading to a total discrepancy of $16.8 \%$ (Fig. 8.22(a)). For the $5 \% \mathrm{Mn}$ doped sample, the RL signal increases by $14.4 \%$ compared to room temperature when the temperature is increased to $100{ }^{\circ} \mathrm{C}$ and decreases by $6.7 \%$ compared to $100{ }^{\circ} \mathrm{C}$ when the temperature is decreased to $40{ }^{\circ} \mathrm{C}$, resulting in a discrepancy of $8.7 \%$ (Fig. 8.22(c)). The increase of the RL signal with increasing temperature for both Mn concentrations might be due to the normal RL trapping and the trapping of deep traps, which was already explained for the Eu doped $\mathrm{RbMgF}_{3}$ nanoparticles. It can be estimated from equation 8.2 . The probability of the trapping, $\tau_{d t}$, of deep traps increases with increasing temperature as shown in Fig. $5.16(\mathrm{~d})$.

The further increase of the RL for the $1 \% \mathrm{Mn}$ sample compared to $100{ }^{\circ} \mathrm{C}$ when the temperature is decreased to $40{ }^{\circ} \mathrm{C}$ was at a slower rate. This could be due to a redistribution of the carriers, which causes a further increase of the trapping rate if shallow traps will be filled during this redistribution and emptied during irradiation at low temperature. Tab. 8.8 summarises the thermal coefficient of the RL signal for the different Mn concentrations in $\mathrm{RbMgF}_{3}$. The thermal coefficient of the RL signal increases with increasing concentration of Mn. It is as low as $0.01 \pm 0.1 \% / \mathrm{K}$ for the $1 \%$ sample, and as high as $0.05 \pm 0.05 \% / \mathrm{K}$ for $5 \% \mathrm{Mn}$ in the nanoparticles. The $\mathrm{RbMgF}_{3}$ bulk materials show a thermal coefficient of the RL of $0.06 \pm 0.1 \%$ for $0.2 \% \mathrm{Mn}$, which is comparable to the values calculated for the nanoparticles.

Table 8.8: Summary of the thermal coefficient of the RL signal for the different doped samples and the $\mathrm{Mn}^{2+}$ ion in $\mathrm{RbMgF}_{3}$ nanoparticles.

\begin{tabular}{|l|l|}
\hline Concentration (\%) & $\mathrm{Mn}^{2+}(\% / \mathrm{K})$ \\
\hline \hline 1 & $0.01 \pm 0.1$ \\
\hline 5 & $0.05 \pm 0.05$ \\
\hline
\end{tabular}




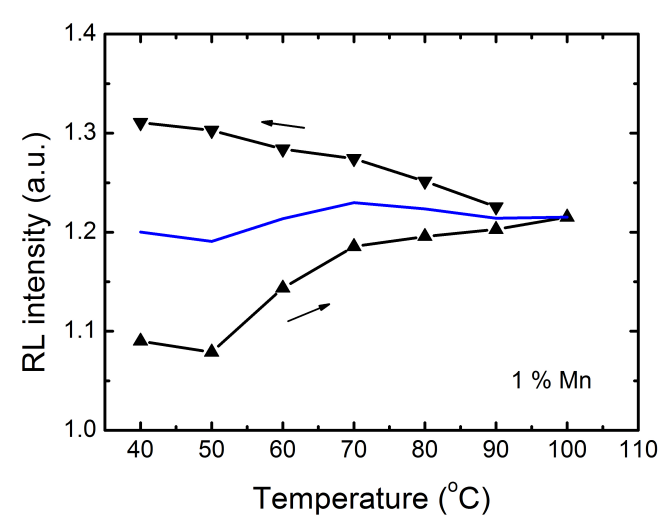

(a)

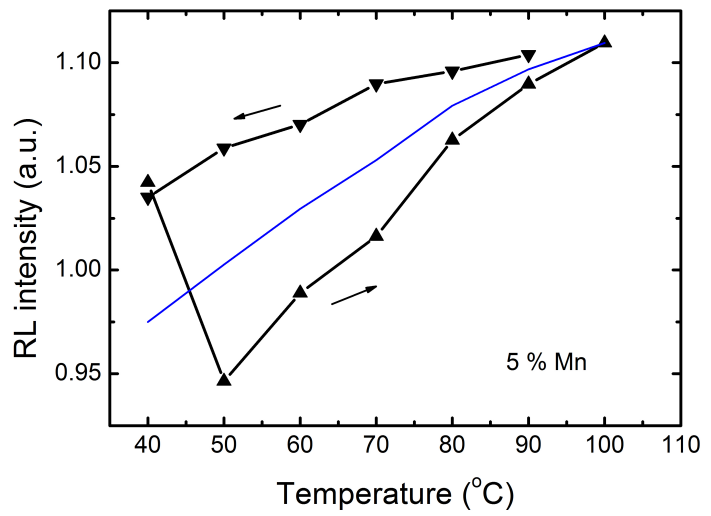

(c)

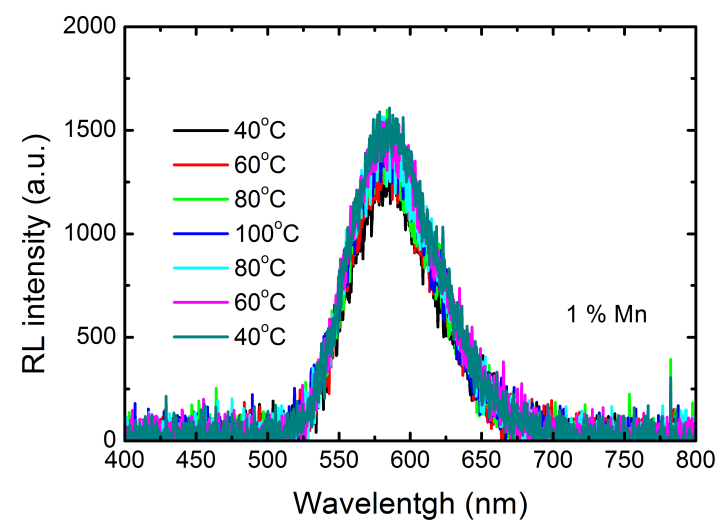

(b)

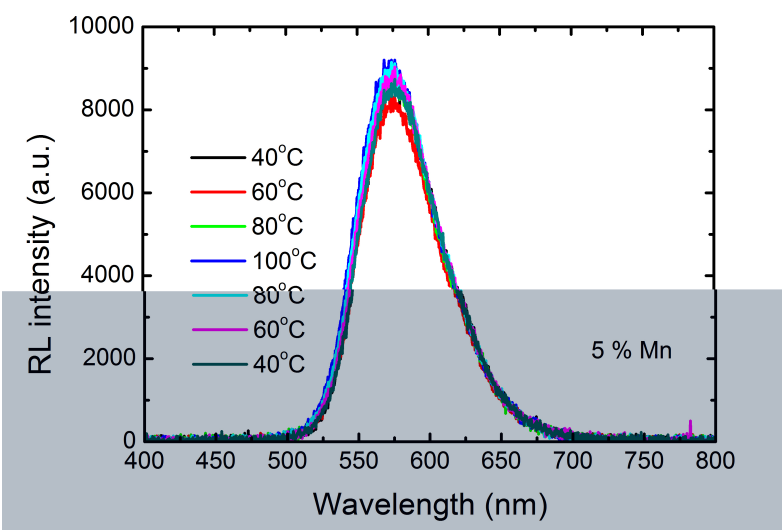

(d)

Figure 8.22: Temperature dependent RL spectra for $\mathrm{RbMgF}_{3}$ nanoparticles doped with 1 $\% \mathrm{Mn}(\mathrm{a})$ and $5 \% \mathrm{Mn}$ (c) for different temperatures up to $100{ }^{\circ} \mathrm{C}$ and the respective RL spectra versus the wavelength for different temperatures ((b) for $1 \% \mathrm{Mn}$ and (d) for $5 \% \mathrm{Mn}$ ).

Arrows facing up signal an increase of temperature, and arrows facing down signal a decrease in temperature. Blue curves show the trend of the temperature dependent RL. 


\subsection{Conclusion}

$\mathrm{Eu}^{2+}, \mathrm{Eu}^{3+}$ and a unknown defect centred around $470 \mathrm{~nm}$ were observed in the PL spectra of the nanoparticles. Temperature dependent PL measurements for the Eu doped samples show that the PL intensity of the unknown defect centred around $470 \mathrm{~nm}$ increases with decreasing temperature. The emission of the unknown defect in the excitation spectra broadens for measurements at low temperature. The intensity of the unknown defect is also dependent of the concentration of the dopant, which means it increases with decreasing Eu concentration. The PL lifetimes of the Eu doped nanoparticles are similar to the bulk materials. $\tau_{12, \text { ave }}$ of the $\mathrm{Eu}^{3+}$ ion increases up to a Eu concentration of $1 \%$ and then decreases again for the $5 \%$ Eu sample. The quantum efficiency was as high as 48.9 \%. Temperature dependent PL lifetime measurements show $\tau_{12 \text {,ave }}$ is nearly constant for decreasing temperature. The PL lifetime of the unknown defect is long and has a value of $57.8 \mathrm{~ms}$ for the $5 \%$ sample and the average lifetime decreases to $54.6 \mathrm{~ms}$ for the 0.1 $\%$ Eu doped sample. It shows thermally activated behaviour with an activation energy of $129 \pm 42 \mathrm{meV}$ for the $1 \%$ doped sample and $108 \pm 44 \mathrm{meV}$ for the $5 \%$ one. The Judd-Ofelt parameters have been calculated as well and $\Omega_{2}$ is in the range found in other nanoparticle compounds. $\Omega_{4}$ is higher than $\Omega_{2}$ and also higher than observed in other compounds. The $\mathrm{RbMgF}_{3}: \mathrm{Eu}$ nanoparticles show comparable TL peaks to the Eu doped bulk materials. The activation energies for these TL peaks measured for the nanoparticles were higher for the bulk materials. $\mathrm{Eu}^{2+}$ and $\mathrm{Eu}^{3+}$ were measured in the RL spectra. The unknown defect centred at $\sim 470 \mathrm{~nm}$ was only observed for the $0.1 \%$ and $0.5 \%$ Eu samples. $\mathrm{I}_{R L}$ is independent of dose from 10 mGy to 100 Gy. The RL intensity decreases for high doses. The integrated RL intensity of the $\mathrm{Eu}^{3+}$ decreases at a slower rate than the $\mathrm{Eu}^{2+} \mathrm{RL}$ intensity which might be due that radiation induced defects preferentially occur neat the $\mathrm{Eu}^{2+}$ sites leading to more quenching of the $\mathrm{Eu}^{2+} \mathrm{RL}$, when compared with the $\mathrm{Eu}^{3+} \mathrm{RL}$. The RL intensity shows a higher dependence of the temperature than the bulk materials. For the $0.1 \% \mathrm{Eu}$ doped nanoparticles, a thermal coefficient for the RL of $0.01 \pm 0.1 \% / \mathrm{K}$ for the $\mathrm{Eu}^{3+}$ ion and of $0.27 \pm 0.2 \% / \mathrm{K}$ for the $\mathrm{Eu}^{2+}$ ion was calculated, which is similar to the 
value calculated for the $0.2 \%$ Eu doped bulk materials $(0.13 \pm 0.1 \% / \mathrm{K})$. For the $1 \%$ doped sample, the thermal coefficient of the RL is $0.24 \pm 0.05 \% / \mathrm{K}$ for the for the $\mathrm{Eu}^{3+}$ ion and $0.07 \pm 0.1 \% / \mathrm{K}$ for the $\mathrm{Eu}^{2+}$ ion. The thermal coefficient of the $\mathrm{RL}$ for the $\mathrm{Eu}^{2+}$ decreases with increasing concentration of $\mathrm{Eu}$ and increases with increasing $\mathrm{Eu}^{3+}$ concentration.

PL measurements of the $\mathrm{Mn}^{2+}$ doped samples show two inequivalent sites of the $\mathrm{Mn}^{2+}$, which were expected from the crystal structure. The two Mn sites were also observed in the $\mathrm{RbMgF}_{3}$ bulk materials. The PL decay of the $\mathrm{Mn}^{2+}$ nanoparticles could be fitted two-exponential for the two regular sites of $\mathrm{Mn}^{2+}$. The $\mathrm{Mn}$ site centred at $550 \mathrm{~nm}$ had a slightly higher lifetime than the Mn centred at higher wavelength, which means this site has a lower concentration of non-radiative sites. TL measurements for the Mn doped $\mathrm{RbMgF}_{3}$ nanoparticles show similar peaks and activation energies as the $\mathrm{NaMgF}_{3}$ : $\mathrm{Mn}$ nanoparticles. $\mathrm{Mn}^{2+}$ is also observed in the RL spectra and the integrated intensity has the same dose dependence as observed for the bulk materials. It increases until $\sim 1 \mathrm{kGy}$ for $5 \% \mathrm{Mn}^{2+}$. Above $1 \mathrm{kGy}$, the RL signal decreases. For the Mn samples doped with $1 \%$ and $2 \%$, the integrated RL intensity decreases also for low doses. The RL intensity is less dependent on the dose as it was observed for the bulk samples, which means that there are different defect distributions in the polycrystalline samples and nanoparticles. This means the nanoparticles show defects on the surface and the bulk materials show non-radiative recombination centres near the RE ion. For the $1 \% \mathrm{Mn}$ doped nanoparticles, the value for the thermal coefficient of the RL was $0.01 \pm 0.1 \% / \mathrm{K}$. For the $5 \% \mathrm{Mn}$ doped sample, the coefficient was as high as $0.05 \pm 0.05 \% / \mathrm{K}$. The thermal coefficient increases with increasing concentration and is comparable to the value, which was observed for the Mn doped bulk materials $(0.06 \pm 0.1 \% / K)$. Next to the luminescent ion there exist additional electronhole-traps. These electrons and holes, which are mobile after irradiation, will more likely recombine on the electron and hole traps and therefore, fewer electrons get trapped in the luminescent ion. The electron and hole traps saturate after an irradiation with a finite dose. Thus, for future temperature dependent RL measurements a finite dose should be 
applied before the measurements. 


\section{Other polycrystalline nanoparticles}

The following chapter gives an overview over measurements performed on other nanoparticles, which have potential to be employed in radiation dosimetry. Fluoride nanoparticles are being actively investigated, because of their wide range of potential applications that include scintillation detectors of ionizing radiation [23, 36] and optical amplifiers [22, 99. In this case, the particular interest is in radiation detectors that are optical transparent, which takes advantage of the reduced scattering that is seen in composites containing nanoparticles [22, 100, 101]. Other advantages of transparent radiation detectors include the potential to detect over a larger volume, when compared with detectors made from bulk compounds, which can result in the ability to detect significantly lower radiation doses. Nanoparticle composites also have the advantage that they can be pressed into arbitrary shapes. Recently, fluoride nanoparticles have been researched for optical amplifiers [22, 99], but reports of the dosimeter properties of these nanoparticles is very limited [23, 24, 36]. In this chapter, the results of the concentration of the dopant on the PL spectra, PL lifetime and RL measurements of fluoride nanoparticles will be discussed.

\subsection{Photoluminescence and PL lifetime measurements of $\mathrm{LaF}_{3}$}

The $\mathrm{LaF}_{3}$ nanoparticles doped with $\mathrm{Sm}^{3+}$ and $\mathrm{Eu}^{3+}$ were prepared using high purity $\mathrm{LaCl}_{3}$, $\mathrm{EuCl}_{3} \times 6 \mathrm{H}_{2} \mathrm{O}, \mathrm{SmCl}_{3} \times 6 \mathrm{H}_{2} \mathrm{O}, \mathrm{NaF}, \mathrm{OA}$, chloroform and ethanol. The nanoparticles capped 
with oleic acid (OA) were prepared with a modified procedure described by Wang et al. [102]. NaF was dissolved in de-ionised water $(90 \mathrm{ml})$ and ethanol $(60 \mathrm{ml})$ and $\mathrm{OA}(20 \mathrm{ml})$ were added to it. The mixture was then stirred and heated up to $75^{\circ} \mathrm{C}$ under an inert atmosphere. $\mathrm{LaCl}_{3}$ and $\mathrm{EuCl}_{3} \times 6 \mathrm{H}_{2} \mathrm{O}$ or $\mathrm{SmCl}_{3} \times 6 \mathrm{H}_{2} \mathrm{O}$ in an aqueous solution was added drop wise to the mixture and stirred at $75{ }^{\circ} \mathrm{C}$ for $5 \mathrm{~h}$. The resultant solution was diluted with ethanol to precipitate the nanoparticles during centrifugation the whole solution at 4 $000 \mathrm{rpm}$ to wash the nanoparticles to remove the residual OA. After repeating this for 5 times the nanoparticles were then dispersed in hexane or chloroform for storage [22].

From XRD measurements, the space group of $\mathrm{LaF}_{3}$ and with the Scherrer formula, the crystal size was obtained. The space group was $\mathrm{P}_{-3 c 1}$ with a crystal size of $\sim 11 \mathrm{~nm}$ [19]. The appearance of the pressed pellets was transparent and the photos of the nanoparticles was already published by Janssens et al. [22, 99]. The powders were pressed into pellets that were $\sim 1 \mathrm{~mm}$ thick where the diameters were $\sim 9 \mathrm{~mm}$ for $\mathrm{Eu}^{3+}$ and $\sim 6 \mathrm{~mm}$ for $\mathrm{Sm}^{3+}$ doped samples [12]. PL spectra and the PL lifetimes were measured using a Varian Cary Eclipse spectrofluorometer, which has a pulsed light source.

\subsubsection{Eu doped $\mathrm{LaF}_{3}$ nanoparticles}

Fig. 9.1 shows the PL excitation spectra (a) and emission spectra (b) for $\mathrm{LaF}_{3}$ doped with $\mathrm{Eu}^{3+}$. The PL excitation spectra shows the PL excitation from the $\lambda_{e m}=590 \mathrm{~nm}$ emission of the $\mathrm{Eu}^{3+}$ ions of the $5 \% \mathrm{Eu}$ doped nanoparticles, which corresponds to the ${ }^{7} \mathrm{~F}_{1}$ level (Fig. 9.1(a)). In Fig. 9.1, the emission spectra for the $\mathrm{Eu}^{3+}$ spectra of the $5 \%$ (b) doped sample and for all of the different $\mathrm{Eu}^{3+}$ concentrations (c) are plotted. The PL emission was excited at $395 \mathrm{~nm}$ and into the intense ${ }^{5} \mathrm{G}_{6}$ and ${ }^{5} \mathrm{~L}_{6}$ levels. It originates mainly from the transitions from the ${ }^{5} \mathrm{D}_{0}$ level, and the transitions from the ${ }^{5} \mathrm{D}_{1}$ become weaker with increasing $\mathrm{Eu}^{3+}$ concentration, which is shown in Fig. 9.1 (c), and can be related to the $\mathrm{Eu}^{3+}$ cross relaxation [84]. The ratio of $\mathrm{I}_{7} F_{2} / \mathrm{I}_{7} F_{1}$ is $0.9: 1$ in the $5 \%$ doped sample. There was no evidence from PL emission measurements for any $\mathrm{Eu}^{2+}$ transitions when excited in 
the ultraviolet [12, 22].

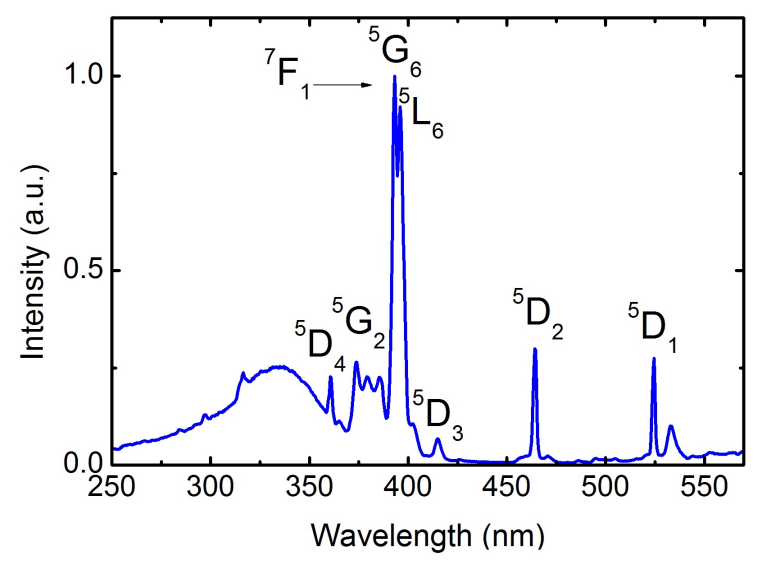

(a)

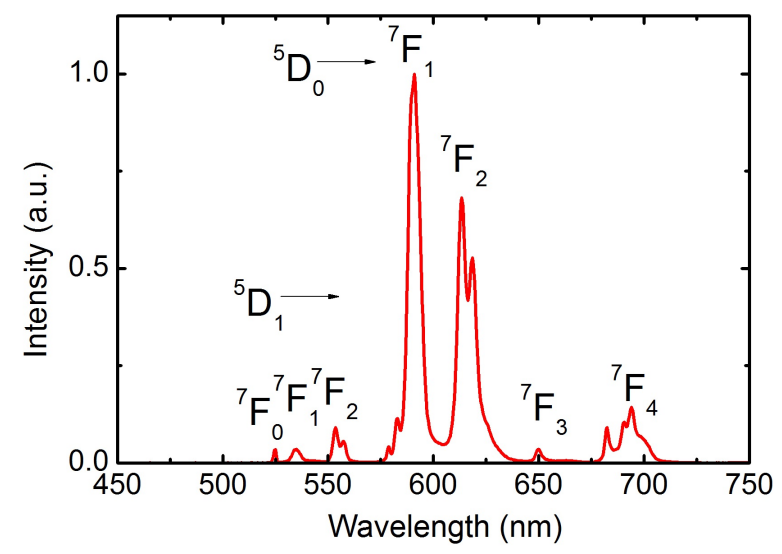

(b)

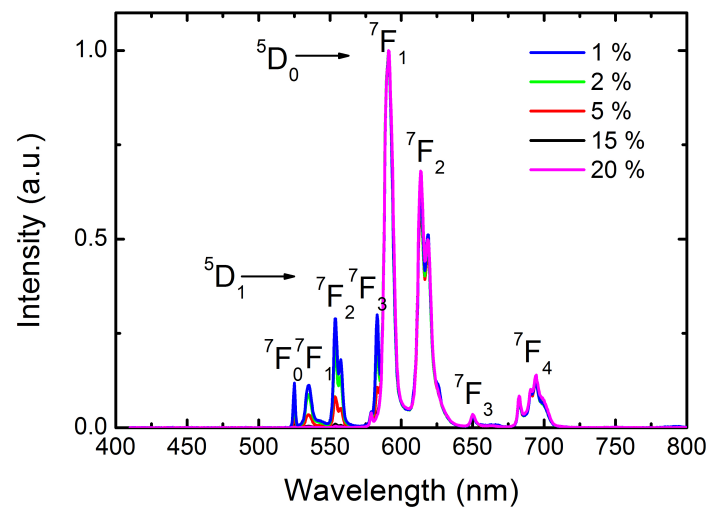

(c)

Figure 9.1: (a) PL excitation $\left(\lambda_{e m}=590 \mathrm{~nm}\right)$ and (b) PL emission $\left(\lambda_{e x}=395 \mathrm{~nm}\right)$ spectra for $\mathrm{LaF}_{3}: 5 \% \mathrm{Eu}^{3+}$ nanoparticles.

(c) PL emission $\left(\lambda_{e x}=395 \mathrm{~nm}\right)$ spectra for all the different $\mathrm{Eu}^{3+}$ concentrations in $\mathrm{LaF}_{3}$ nanoparticles [12].

Fig. 9.2 shows the average PL lifetime of $\mathrm{LaF}_{3}$ doped with different concentrations of $\mathrm{Eu}^{3+}$ and a lifetime decay curve for $5 \% \mathrm{Eu}$. The PL decay could be fitted to two PL lifetimes, and $\tau_{\text {ave }}$ was obtained from $\left.\tau_{\text {ave }}=\left[\int t \mathrm{x} I(t) d t\right] / \int I(t) d t\right]$. Fig. 9.2 shows the average PL lifetimes, $\tau_{\text {ave }}$, for the $\mathrm{LaF}_{3}$ nanoparticles doped with different concentrations of $\mathrm{Eu}^{3+}$. The average PL lifetime decreases with increasing $\mathrm{Eu}^{3+}$ concentration [12]. The 
decrease in $\tau_{\text {ave }}$ with increasing $\mathrm{Eu}^{3}$ concentration has been previously reported for the other nanoparticles $\left(\mathrm{RbMgF}_{3}\right.$ and $\left.\mathrm{NaMgF}_{3}\right)$, and can be attributed to energy transfer from $\mathrm{Eu}^{3+}$ sites away from the surface to $\mathrm{Eu}^{3+}$ sites near the surface followed by non-radiative decay at unidentified surface non-radiative recombination sites [10, 11, 12, 22]. This energy transfer process can occur via a dipole-dipole or a dipole-quadrupole interaction [91, and hence it will increase with increasing $\mathrm{Eu}^{3+}$ concentration. The $\mathrm{Eu}^{3+} \mathrm{PL}$ lifetime will decrease until it reaches the PL lifetime of $\mathrm{Eu}^{3+}$ near the surface [12]. The radiative lifetime was calculated with a different method than explained for the $\mathrm{NaMgF}_{3}$ : Eu and $\mathrm{RbMgF}_{3}$ nanoparticles. In the method which was used for the other nanoparticles the radiative lifetime was estimated from [68],

$$
\tau_{\text {rad }}=\frac{1}{\sum_{J} A_{0-J}}
$$

where $\mathrm{A}_{0-J}$ are the 0-J transition rates calculated with the Judd-Ofelt theory. In this method the radiative lifetime, $\tau_{r a d}$, was estimated from [103],

$$
\frac{1}{\tau_{\text {rad }}}=A_{M D} n^{3} \frac{I_{t o t}}{I_{M D}}
$$

where $n$ is the effective refractive index of the pressed nanoparticles. In this case it is the average of the refractive indices of the nanocrystalline material $\mathrm{n}_{\mathrm{LFF}_{3}}(=1.59)$ and the surrounding media $\mathrm{n}_{O A}(=1.459)$, which leads to

$$
n=x \times n_{L F_{3}}+(1-x) n_{O A}
$$

where $\mathrm{x}(=0.4)$ is the nanoparticle volume fraction. $\mathrm{A}_{M D}\left(=14.65 \mathrm{~s}^{-1}\right)$ is the spontaneous emission probability for the magnetic dipole ${ }^{5} \mathrm{D}_{0} \rightarrow{ }^{7} \mathrm{~F}_{1}$ transition in vacuo. $\mathrm{I}_{t o t}$ and $\mathrm{I}_{M D}$ are the total and the ${ }^{5} \mathrm{D}_{0} \rightarrow{ }^{7} \mathrm{~F}_{1}$ integrated emission intensity of the corrected $\mathrm{Eu}^{3+}$ emission spectra, respectively. It is relatively easy to calculate the $\mathrm{Eu}^{3+}$ quantum efficiency,

$$
\eta=\frac{\tau_{\text {ave }}}{\tau_{\text {rad }}}
$$

with the known radiative lifetime, $\tau_{\text {rad }}$. It was calculated that $\tau_{\text {rad }}$ is $(7.2 \pm 0.7) \mathrm{ms}$, which is greater than the measured PL lifetimes. The resultant quantum efficiencies are listed in Tab. 9.1 and the quantum efficiency was as high as $79 \%$ [19]. 


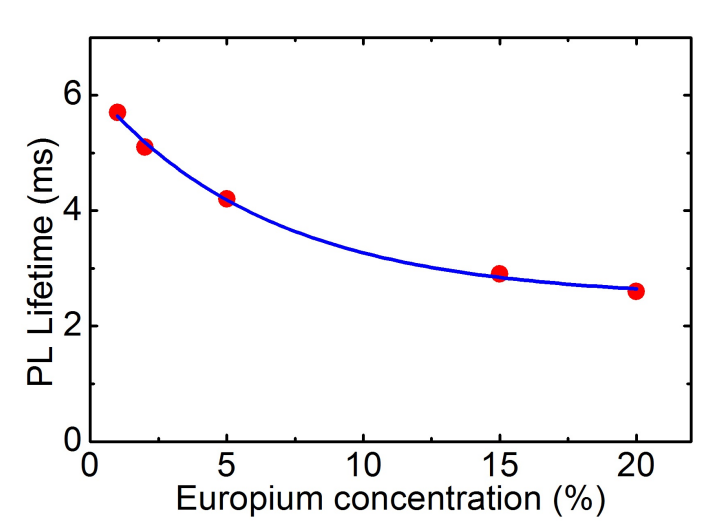

(a)

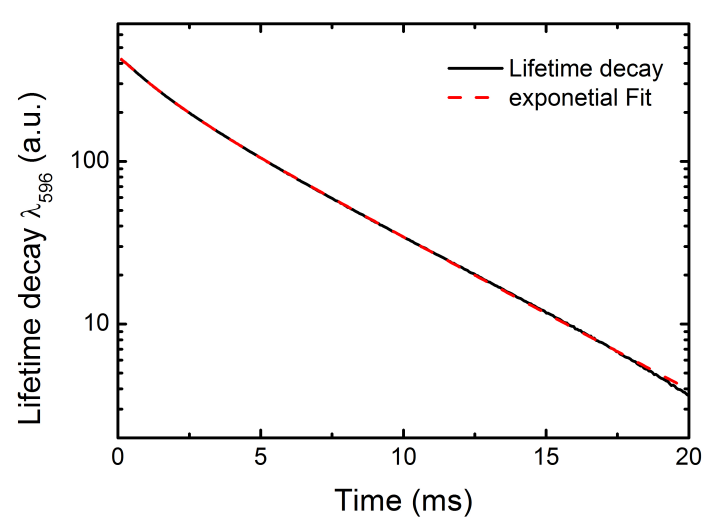

(b)

Figure 9.2: (a) Average PL lifetime for $\mathrm{Eu}^{3+}\left(\lambda_{e m}=590 \mathrm{~nm}, \lambda_{e x}=400 \mathrm{~nm}\right)$ doped $\mathrm{LaF}_{3}$ samples against the rare earth concentration.

The line is guided to the eye [12].

(b) PL lifetime decay of $5 \% \mathrm{Eu}$ doped $\mathrm{LaF}_{3}$ at $\lambda_{\text {em }}=590 \mathrm{~nm}$ (black curve). The red curve is the exponential fit. The lifetime was measured at $\lambda_{e m}=400$ nm.

Janssens et al. reported in a recent paper PL and PL lifetime studies of Eu doped $\mathrm{LaF}_{3}$ nanoparticles. Their average PL lifetime was calculated with a method described in chapter 3.2.4 but the PL lifetimes were the same as calculated with this method. Janssens et al. already showed extensive studies of the PL decay curves in their paper as well as in his Ph.D. thesis. Hence, no further PL lifetime curves will be discussed as it was already published [19, 22]. 
Table 9.1: Summary of different measured PL lifetimes, $\tau_{\text {ave }}$ and the quantum efficiency, $\eta$, for $\mathrm{LaF}_{3}$ doped with different concentrations of $\mathrm{Eu}^{3+}[12]$.

\begin{tabular}{|l|l|l|}
\hline Concentration (\%) & $\tau_{\text {ave }}(\mathrm{ms})$ & $\eta(\%)$ \\
\hline \hline 1 & $5.7 \pm 0.6$ & 79 \\
\hline 2 & $5.1 \pm 0.5$ & 71 \\
\hline 5 & $4.2 \pm 0.4$ & 58 \\
\hline 15 & $2.9 \pm 0.3$ & 40 \\
\hline 20 & $2.6 \pm 0.3$ & 36 \\
\hline
\end{tabular}

Janssens et al. reported in their paper similar quantum efficiencies. The quantum efficiency was as high as $83.8 \%$ and their calculated $\Omega_{2}$ and $\Omega_{4}$ were in the range of (1.6 - 1.9) $\times 10^{-20} \mathrm{~cm}^{2}$ and $(2.5-3.6) \times 10^{-20} \mathrm{~cm}^{2}$, respectively [22]. It was observed that $\Omega_{2}$ is larger than $\Omega_{4}$, which was also reported for the $\mathrm{NaMgF}_{3}$ and $\mathrm{RbMgF}_{3}$ nanoparticles in this thesis. There was no dependence of $\Omega_{2}$ and $\Omega_{4}$ on the concentration of the rare earth observed. Higher values for $\Omega_{2}$ and $\Omega_{4}$ for the TTA/OA (TTA=thenoyltrifluoroacetone) capped nanoparticles were reported by Janssens et al.. $\Omega_{2}$ and $\Omega_{4}$ were in ranges of (4.1-8.7) $\times 10^{-20} \mathrm{~cm}^{2}$ and (3.0-4.7) $\times 10^{-20} \mathrm{~cm}^{2}$, respectively. A decrease of $\Omega_{2}$ and $\Omega_{4}$ with increasing $\mathrm{Eu}^{3+}$ concentration was investigated [22]. As already mentioned in the previous chapters, $\Omega_{2}$ is higher for more distorted sites, which means that low concentrations of $\mathrm{Eu}^{3+}$ in the TTA/OA capped materials show a higher distorted site of $\mathrm{Eu}^{3+}$ [10, 93].

\subsubsection{Sm doped $\mathrm{LaF}_{3}$ nanoparticles}

The PL emission and excitation spectra for $\mathrm{LaF}_{3}$ nanoparticles doped with $5 \% \mathrm{Sm}^{3+}$ are shown in Fig. 9.3(a) and Fig. 9.3(b). The PL emission was excited into the $\mathrm{Sm}^{3+}{ }^{7} \mathrm{~F}_{5 / 2}$ level $(400 \mathrm{~nm})$ and the emission for the excitation spectra was detected at $600 \mathrm{~nm}$, which 
corresponds to the $\mathrm{Sm}^{3+}{ }^{6} \mathrm{H}_{7 / 2}$ level. The PL emission occurs via initial non-radiative decay to the ${ }^{4} \mathrm{G}_{5 / 2}$ level. $\mathrm{Sm}^{2+}$ can sometimes occur in nanoparticles even when $\mathrm{Sm}^{3+}$ is in the starting compounds [19, 104]. For this reason, PL measurements were also done by exciting in the ultraviolet and there was no evidence for any $\mathrm{Sm}^{2+}$.

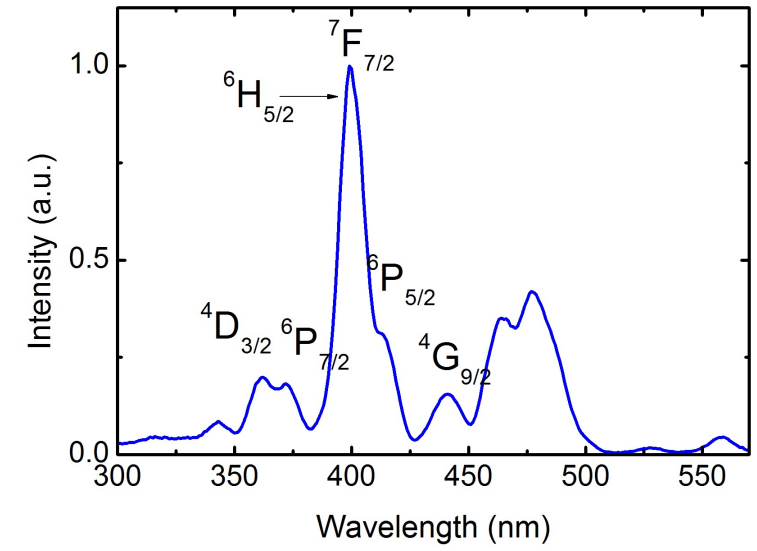

(a)

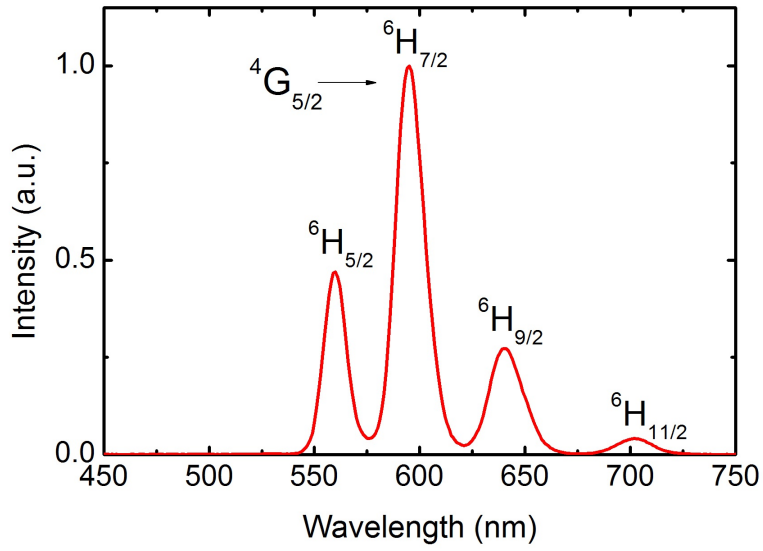

(b)

Figure 9.3: (a) PL excitation $\left(\lambda_{e m}=600 \mathrm{~nm}\right)$ and (b) PL emission $\left(\lambda_{e x}=400 \mathrm{~nm}\right)$ spectra for $\mathrm{LaF}_{3}: 5 \% \mathrm{Sm}^{3+}$ nanoparticles [12].

For the $\mathrm{Eu}^{3+}$ doped $\mathrm{LaF}_{3}$, it was shown that the decrease in $\tau_{\text {ave }}$ with increasing $\mathrm{Eu}^{3}$ concentration can be attributed to energy transfer from $\mathrm{Eu}^{3+}$ sites away from the surface to $\mathrm{Eu}^{3+}$ sites near the surface followed by non-radiative decay at unidentified surface nonradiative recombination sites $[10,11,12,22]$. A similar energy transfer process is also likely for $\mathrm{Sm}^{3+}$. However, cross-relaxation to neighbouring $\mathrm{Sm}^{3+}$ sites can also occur [105]. For example, excitation followed by non-radiative decay to the ${ }^{4} \mathrm{G}_{5 / 2}$ level can result in a ${ }^{4} \mathrm{G}_{5 / 2}$ $\rightarrow{ }^{6} \mathrm{~F}_{5 / 2}$ transition on one $\mathrm{Sm}^{3+}$ site, and a ${ }^{6} \mathrm{H}_{5 / 2} \rightarrow{ }^{6} \mathrm{~F}_{11 / 2}$ transition on the neighbouring $\mathrm{Sm}^{3+}$ (Fig. 9.4). 


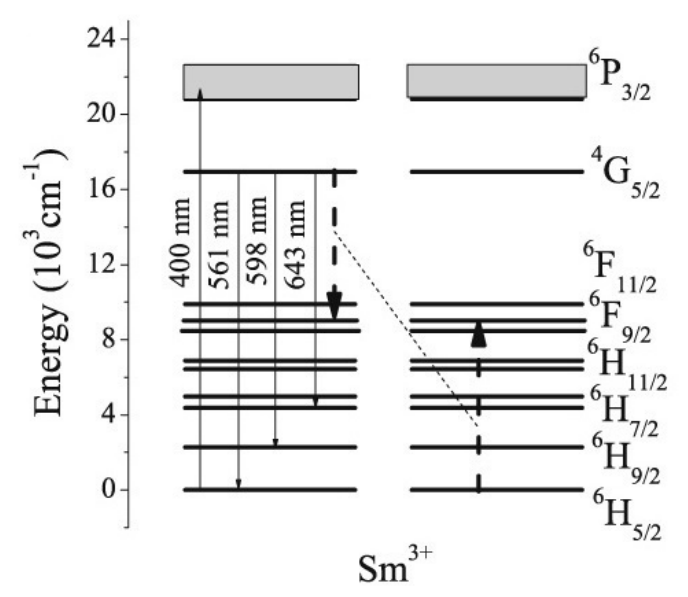

Figure 9.4: Cross relaxation process between $\mathrm{Sm}^{3+}$ ion [13].

This leaves one $\mathrm{Sm}^{3+}$ ion in the ${ }^{6} \mathrm{~F}_{5 / 2}$ level and the other $\mathrm{Sm}^{3+}$ ion in the ${ }^{6} \mathrm{~F}_{11 / 2}$ level followed by non-radiative decay. This leads to two possible mechanisms that can cause the $\mathrm{Sm}^{3+}$ PL lifetime to decrease. The first energy transfer mechanism to $\mathrm{Sm}^{3+}$ sites near the surface followed by non-radiative decay should result in a PL lifetime that decreases and reaches a constant value for high concentrations. This is observed in Fig. 9.5(a). The second mechanism where there is cross relaxation and non-radiative decay might be expected to continually decrease with increasing $\mathrm{Sm}^{3+}$ concentration. This appears to be inconsistent with the data plotted in Fig. 9.5(a), and hence the first mechanism is probably the main process. Tab. 9.2 shows the summary of the measured average PL lifetimes, $\tau_{\text {ave }}$, for $\mathrm{LaF}_{3}$ doped with different concentrations of $\mathrm{Sm}^{3+}[12]$. 


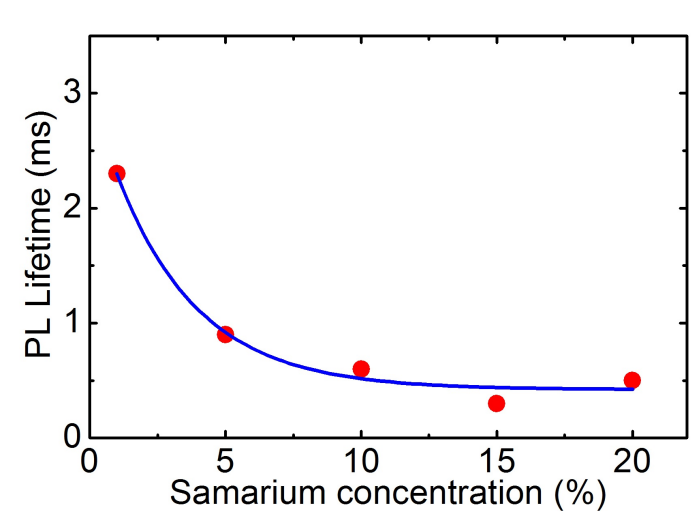

(a)

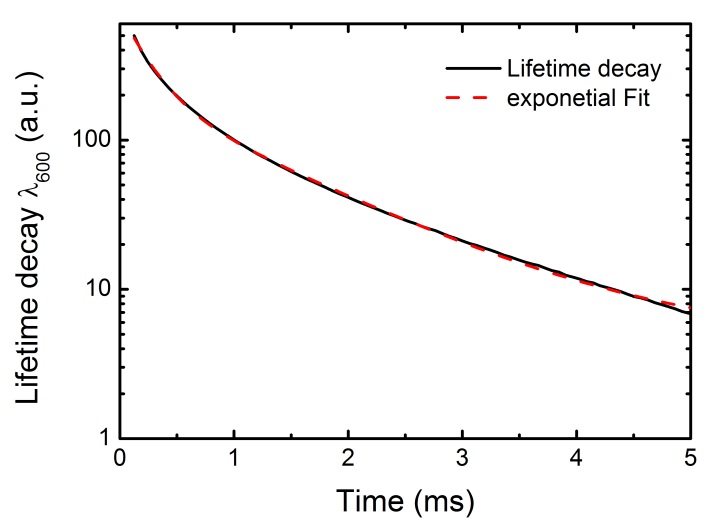

(b)

Figure 9.5: (a) Average PL lifetimes for $\mathrm{Sm}^{3+}\left(\lambda_{e m}=600 \mathrm{~nm}, \lambda_{e x}=400 \mathrm{~nm}\right)$ doped $\mathrm{LaF}_{3}$ samples against the rare earth concentration. The line is guided to the eye [12]. (b) PL lifetime decay of $5 \% \mathrm{Sm}$ doped $\mathrm{LaF}_{3}$ at $\lambda_{e m}=600 \mathrm{~nm}$ (black curve). The red curve is the exponential fit. The lifetime was measured at $\lambda_{e m}=400$ nm.

A detailed study of the PL and PL lifetime measurements including the PL decay curves was already published in the Ph.D. thesis of Stefaan Janssens and will not be repeated in this thesis [19].

Table 9.2: Summary of the different measured PL lifetimes, $\tau_{\text {ave }}$, for $\mathrm{LaF}_{3}$ doped with different concentrations of $\mathrm{Sm}^{3+}$.

\begin{tabular}{|l|l|}
\hline Concentration (\%) & $\tau_{\text {ave }}(\mathrm{ms})$ \\
\hline \hline 1 & $2.3 \pm 0.2$ \\
\hline 5 & $0.9 \pm 0.1$ \\
\hline 10 & $0.6 \pm 0.1$ \\
\hline 15 & $0.30 \pm 0.03$ \\
\hline 20 & $0.5 \pm 0.1$ \\
\hline
\end{tabular}




\subsection{Radioluminescence measurements of $\mathrm{LaF}_{3}$}

The RL measurements were performed during continuous $60 \mathrm{keV} \gamma$-ray irradiation using a ${ }^{241} \mathrm{Am}$ source or during exposure with X-rays generated by a cobalt X-ray tube $(40 \mathrm{kV}$ and $40 \mathrm{~mA}$ ) with an Aluminium filter. The ${ }^{241} \mathrm{Am}$ RL measurements were performed using a custom-built system, where the ${ }^{241} \mathrm{Am}$ was placed on top of the sample that was located on top of a photomultiplier tube. Higher dose measurements were constructed employing a custom-built system attached to an X-ray set where the sample was located $\sim 3 \mathrm{~cm}$ from the exit port. This system was also used to collect the RL spectra where an optical fibre was connected to an Ocean Optics USB 4000 spectrometer that had a CCD detector array.

\subsubsection{Eu doped $\mathrm{LaF}_{3}$ nanoparticles}

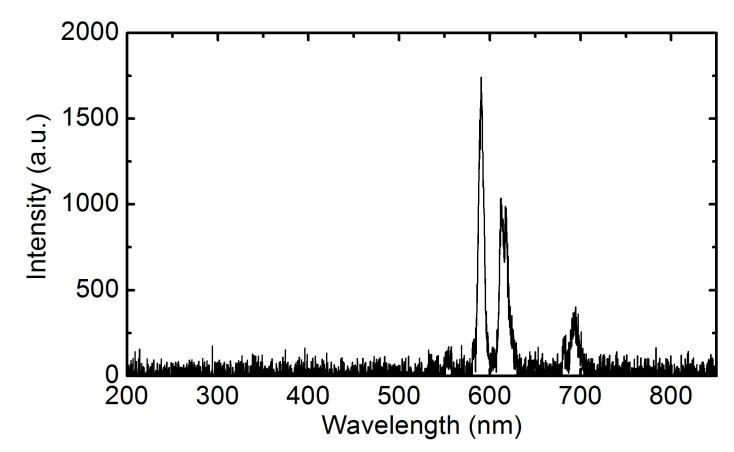

(a)

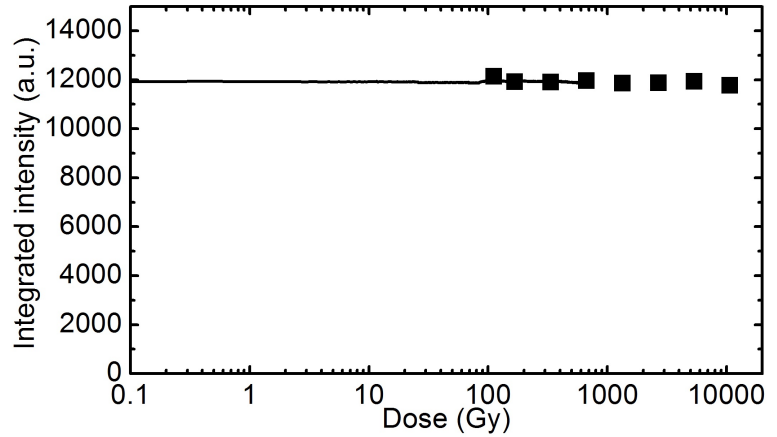

(b)

Figure 9.6: (a) Radioluminescence spectra for $\mathrm{LaF}_{3}: 5 \% \mathrm{Eu}^{3+}$ nanoparticles during continuous X-ray irradiation.

(b) The integrated RL spectra as a function of irradiation time for $\mathrm{LaF}_{3}: 5 \%$ $\mathrm{Eu}^{3+}$ nanoparticles during continuous X-ray irradiation (filled squares) or 60 $\mathrm{keV} \gamma$-ray irradiation (solid line).

The dashed line is a guide to the eye. 
Fig. 9.6(a) shows the RL spectra during continuous X-ray excitation for a $5 \% \mathrm{Eu}^{3+}$ sample. The RL can be completely attributed to $\mathrm{Eu}^{3+}$ and there are no $\mathrm{Eu}^{2+}$ emissions. The energies of the $\mathrm{Eu}^{3+}$ emissions are the same as those observed in the PL spectra. The integrated RL intensity is plotted in Fig. 9.6(b) (filled squares), and it can be observed that it is independent of dose up to $\sim 10 \mathrm{kGy}$. It was not possible to perform similar measurements at lower doses, because the sensitivity of the CCD detector was not high enough. For this reason low dose measurements were performed with a ${ }^{241} \mathrm{Am}$ source. The resultant RL intensity is plotted in Fig. 9.6(b) (solid line), and scaled to match the data obtained from X-ray irradiation. It is apparent that the RL is linear even for low doses where a dose dependence might be expected if there exists electron and hole traps that become filled during irradiation [73]. Thus, it was found that the RL signal is linear from 0.1 Gy to $10 \mathrm{kGy}$ and spanning 5 orders of magnitude. This is advantageous for real time dose monitoring, because other compounds can be damaged when exposed to doses in the kGy region and they can also display a dose-dependent RL. The RL signal decreases by $2.3 \%$ at $1 \mathrm{kGy}$ and by $3.0 \%$ at $10 \mathrm{kGy}$. The ratio $\mathrm{I}_{{ }^{7} F_{2}} / \mathrm{I}_{7} F_{1}$ is $0.9: 1$ in the $5 \%$ doped sample, which was the same as observed in the PL spectra.

\subsubsection{Sm doped $\mathrm{LaF}_{3}$ nanoparticles}

The RL spectra for the $5 \% \mathrm{Sm}^{3+}$ sample is plotted in Fig. 9.7(a), and it contains only $\mathrm{Sm}^{3+}$ emissions where the energy of the emissions are the same as those observed in the PL spectra. Figure 9.7(b) shows the integrated RL intensity plotted against the X-ray dose. It is apparent that the RL is independent of the dose up to $\sim 5 \mathrm{kGy}$, and there in only a very small increase by $5.3 \%$ in the RL intensity at $10 \mathrm{kGy}$ [12]. 


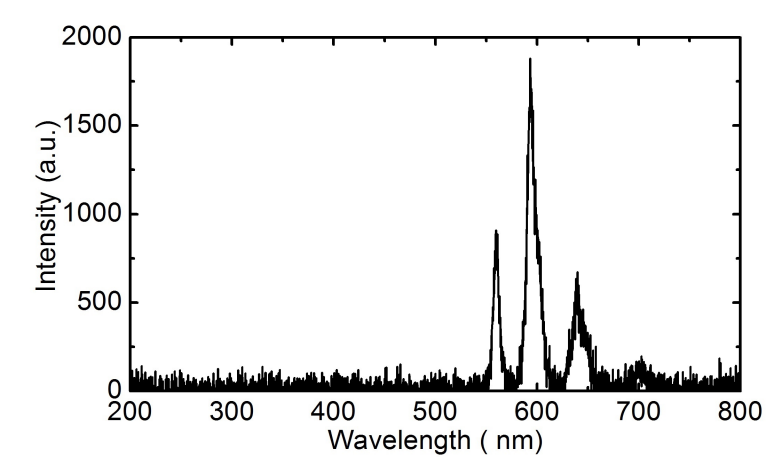

(a)

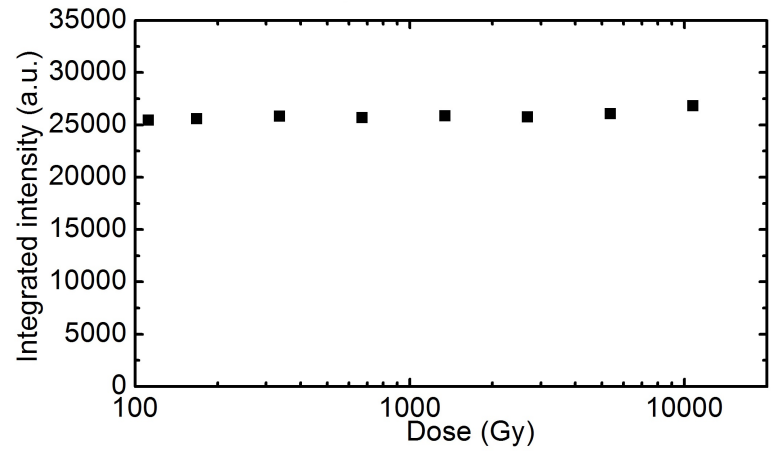

(b)

Figure 9.7: (a) Radioluminescence spectra for (a) $\mathrm{LaF}_{3}: 5 \% \mathrm{Sm}^{3+}$ nanoparticles.

(b) The integrated RL spectra as a function of irradiation time for $\mathrm{LaF}_{3}: 5 \%$ $\mathrm{Sm}^{3+}$ nanoparticles. The radioluminescence data were recorded during continuous X-ray irradiation.

\subsection{Photoluminescence measurements of $\mathrm{BaF}_{2}$}

$\mathrm{BaF}_{2}$ nanoparticles doped with $5 \% \mathrm{Eu}^{3+}$ were produced with a reverse micro-emulsion method similar to producing the $\mathrm{NaMgF}_{3}$ and $\mathrm{RbMgF}_{3}$ nanoparticles. The precursors were high purity $\mathrm{BaNO}_{3}, \mathrm{Eu}\left(\mathrm{NO}_{3}\right)_{3}, \mathrm{NH}_{4} \mathrm{~F}$ and $\mathrm{CTAB}$. BaF 2 has a cubic crystal structure and the crystal size was measured from the XRD data with the Scherrer formula and the nanoparticles had an average diameter of $\sim 12 \mathrm{~nm}$ [26]. PL measurements were performed with the Cary50. $\mathrm{BaF}_{2}$ were doped with $5 \% \mathrm{Eu}$ and the PL excitation and emission spectra is plotted in Fig. 9.8. The PL excitation spectra was measured at an emission wavelength of $590 \mathrm{~nm}$ and it consists only the $\mathrm{Eu}^{3+}$ emission from the ${ }^{7} \mathrm{~F}_{1}$ level. The PL emission spectra was excited at $395 \mathrm{~nm}$, which are into the intense ${ }^{5} \mathrm{G}_{6}$ and ${ }^{5} \mathrm{~L}_{6}$ levels. The PL emission originates mainly from the ${ }^{5} \mathrm{D}_{0}$ transitions. The ${ }^{5} \mathrm{D}_{1}$ transitions are very weak (Fig. $9.8(\mathrm{~b}))$. 


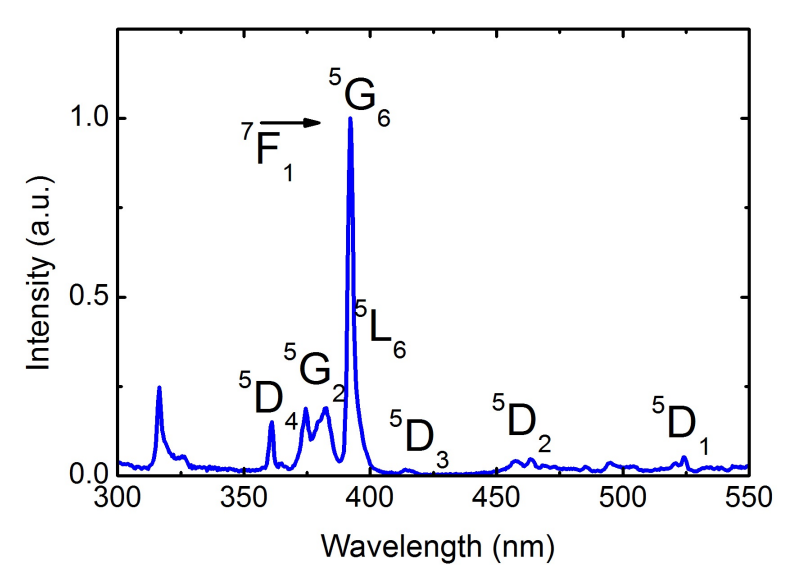

(a)

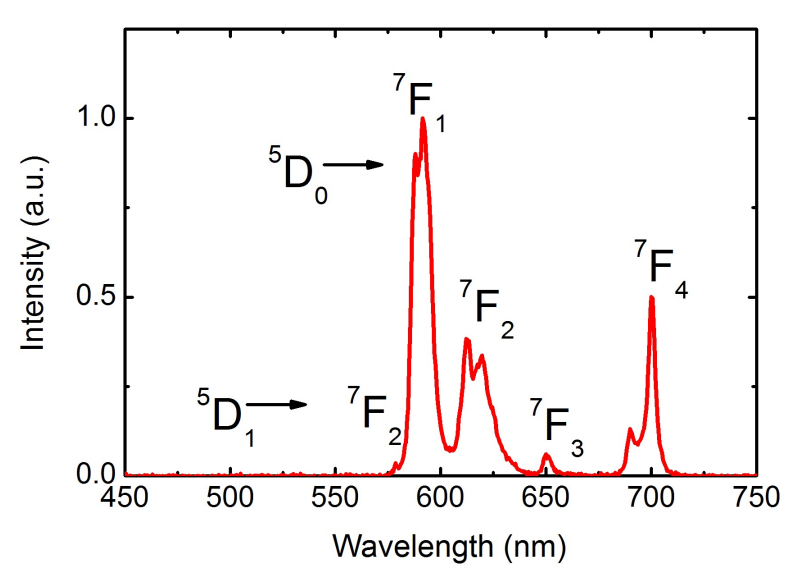

(b)

Figure 9.8: (a) PL excitation $\left(\lambda_{e m}=590 \mathrm{~nm}\right)$ and (b) PL emission $\left(\lambda_{e x}=395 \mathrm{~nm}\right)$ spectra for $\mathrm{BaF}_{2}: 5 \% \mathrm{Eu}^{3+}$ nanoparticles.

\subsection{Radioluminescence measurements of $\mathrm{BaF}_{2}$}

RL measurements were performed with X-rays generated by a cobalt X-ray tube $(40 \mathrm{kV}$ and $40 \mathrm{~mA}$ ) with an Aluminium filter, and RL was detected with a CCD detector. In Fig. 9.9 (a), the RL spectra for $\mathrm{BaF}_{2}: 5 \% \mathrm{Eu}^{3+}$ nanoparticles during continuous X-ray irradiation, is plotted. Fig. 9.9(b) shows the integrated RL spectra as a function of irradiation time during continual X-ray irradiation. The RL emission can be completely contributed to the $\mathrm{Eu}^{3+}{ }^{5} \mathrm{D}_{0}$ and ${ }^{5} \mathrm{D}_{1}$ transitions with $\mathrm{RL}$ emissions being the same as observed in the PL spectra (Fig. 9.9(a)). There is no shift in the RL or PL emission wavelength after heavily irradiation. Fig. 9.9(b) shows the integrated RL intensity plotted against the X-ray dose for $5 \% \mathrm{Eu}^{3+}$ and the RL is nearly independent of dose up to $\sim 10$ kGy. The RL intensity increases by $3.9 \%$ at $\sim 1 \mathrm{kGy}$ and decreases by $1.3 \%$ at $\sim 7 \mathrm{kGy}$. 


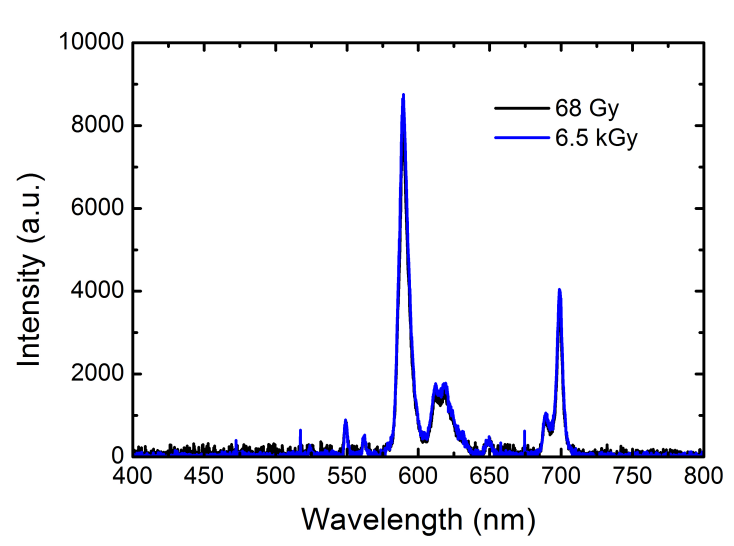

(a)

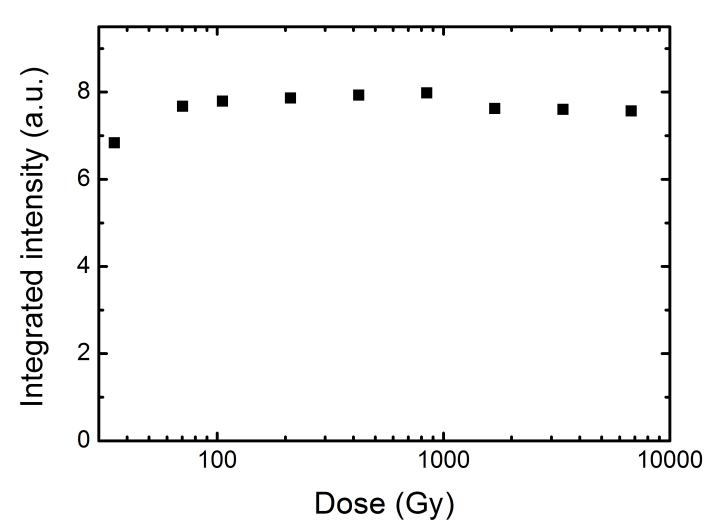

(b)

Figure 9.9: (a) Radioluminescence spectra for $\mathrm{BaF}_{2}: 5 \% \mathrm{Eu}^{3+}$ nanoparticles during continuous X-ray irradiation after 68 Gy (black line) and 6.5 kGy (blue line).

(b) The integrated RL spectra as a function of irradiation time for $\mathrm{BaF}_{2}: 5 \%$ $\mathrm{Eu}^{3+}$ nanoparticles during continuous X-ray irradiation.

\subsection{Photoluminescence measurements of $\mathrm{BaMgF}_{4}$}

$\mathrm{BaMgF}_{4}$ doped with $5 \% \mathrm{Mn}^{2+}$ were produced with a reverse micro-emulsion method similar to that used to the method to produce the other nanoparticles, i.e. $\mathrm{NaMgF}_{3}$, $\mathrm{RbMgF}_{3}$ and $\mathrm{BaMgF}_{4}$ nanoparticles. The precursors were high purity $\mathrm{BaNO}_{3}, \mathrm{MgNO}_{3}$, $\mathrm{Mn}\left(\mathrm{NO}_{3}\right)_{3}, \mathrm{NH}_{4} \mathrm{~F}$ and $\mathrm{CTAB}$. BaMgF 4 has a orthorhombic crystal structure and the crystal size was measured from the XRD data with the Scherrer formula and the nanoparticles had an average diameter of $\sim 11 \mathrm{~nm}[19$, 77]. PL measurements were performed with the Jobin-Yvon FluoroLog ${ }^{\circledR}$. $\mathrm{Mn}^{2+}$ can substitute for two lattice sites in $\mathrm{BaMgF}_{4}$, the $\mathrm{Ba}^{2+}$ and the $\mathrm{Mg}^{2+}$ site. An emission at $630 \mathrm{~nm}$ was detected which corresponds to the $\mathrm{Mg}^{2+}$ site [81. $\mathrm{BaMgF}_{4}$ were doped with $5 \% \mathrm{Mn}^{2+}$ and the PL excitation and emission spectra is plotted in Fig. 9.10. The PL excitation spectra were measured at an emission wavelength of $630 \mathrm{~nm}$ and it shows only the $\mathrm{Mn}^{2+}$ emission of the ${ }^{6} \mathrm{~A}_{1 g}$ level, which are indicated in Fig. 9.10(a). The PL emission spectra was excited at $396 \mathrm{~nm}$, which are into the intense 
${ }^{4} \mathrm{E}_{g}$ and ${ }^{4} \mathrm{~A}_{1 g}$ levels (Fig. 9.10(b)). The PL emission consists of one peak at $630 \mathrm{~nm}$. There are no additional peaks from a highly distorted $\mathrm{Mn}^{2+}$ site detected in the PL emission spectrum as it was observed for the $\mathrm{NaMgF}_{3}: \mathrm{Mn}$ bulk and nanoparticles.

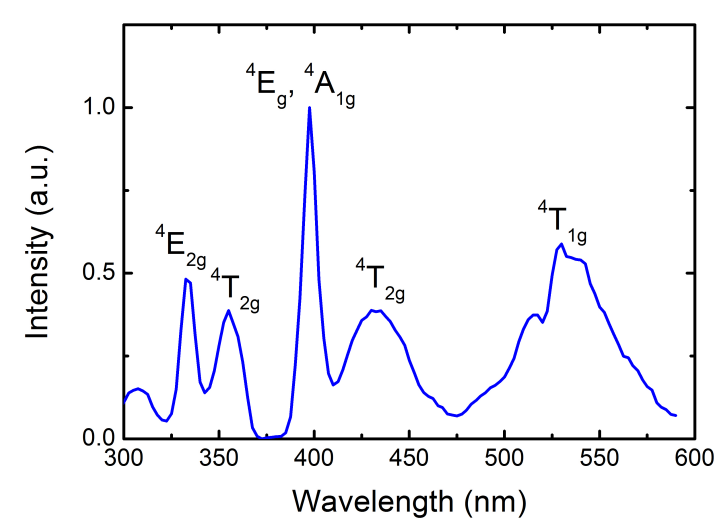

(a)

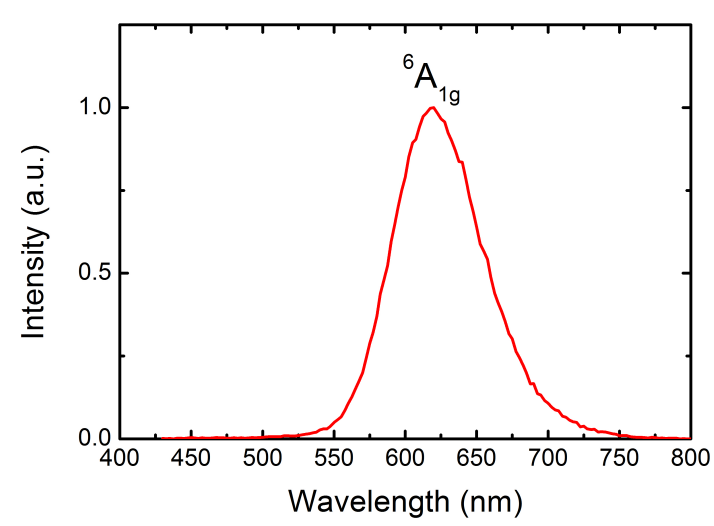

(b)

Figure 9.10: (a) PL excitation $\left(\lambda_{e m}=630 \mathrm{~nm}\right)$ and (b) PL emission $\left(\lambda_{e x}=396 \mathrm{~nm}\right)$ spectra for $\mathrm{BaMgF}_{4}: 5 \% \mathrm{Mn}^{2+}$ nanoparticles.

\subsection{Radioluminescence measurements of $\mathrm{BaMgF}_{4}$}

RL measurements were performed with X-rays generated by a cobalt X-ray tube $(40 \mathrm{kV}$ and $40 \mathrm{~mA}$ ) with an Aluminium filter and RL was detected with a CCD detector. In Fig. 9.11(a), the RL spectra for $\mathrm{BaMgF}_{4}: 5 \% \mathrm{Mn}^{2+}$ nanoparticles during X-ray irradiation is plotted. Fig. 9.11(b) shows the integrated RL spectra as a function of irradiation time during continuous X-ray irradiation. The RL emission can be completely contributed to the $\mathrm{Mn}^{2+}$ emission at $\sim 630 \mathrm{~nm}$ with $\mathrm{RL}$ emissions being the same as observed in the PL spectra (Fig. 9.11(a)). There is no shift in the RL or PL emission wavelength after heavily irradiation. Fig. 9.11(b) shows the integrated RL intensity plotted against the X-ray dose for $5 \% \mathrm{Mn}^{3+}$ and the RL is increases by $7.3 \%$ at $1 \mathrm{kGy}$ and increases by $17.4 \%$ at $\sim 10$ kGy. 


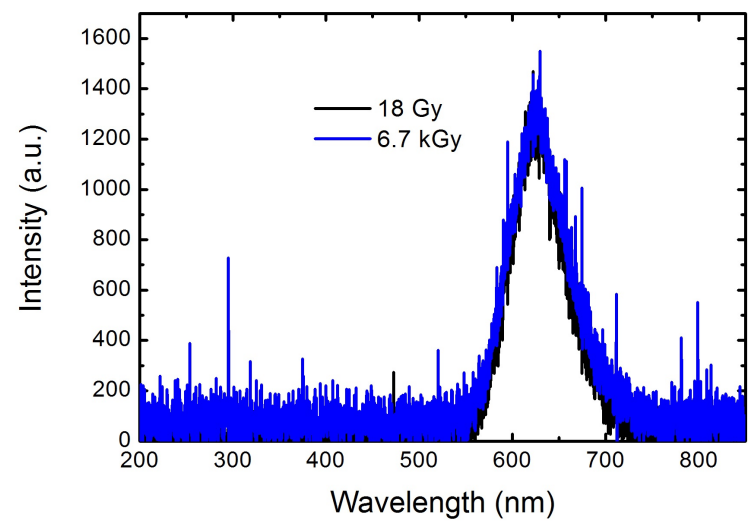

(a)

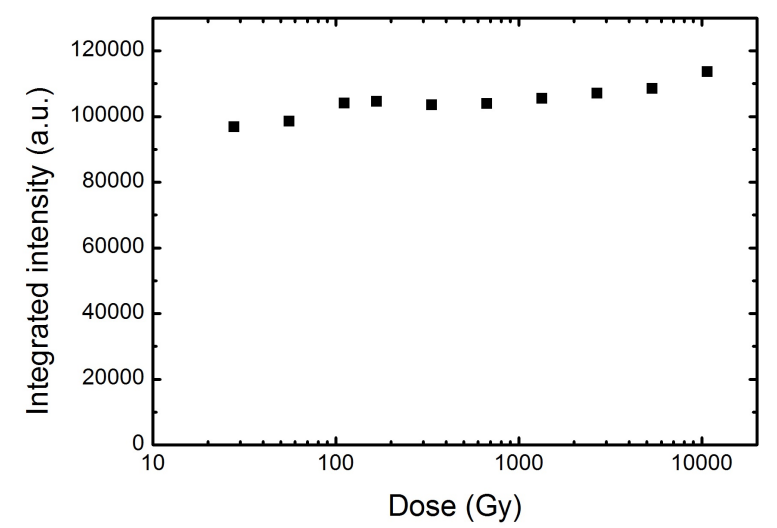

(b)

Figure 9.11: (a) Radioluminescence spectra for $\mathrm{BaMgF}_{4}: 5 \% \mathrm{Mn}^{2+}$ nanoparticles during continuous X-ray irradiation after 68 Gy (black line) and 6.5 kGy (blue line).

(b) The integrated RL spectra as a function of irradiation time for $\mathrm{BaMgF}_{4}$ : $5 \% \mathrm{Mn}^{2+}$ nanoparticles during continuous X-ray irradiation.

\subsection{Conclusion}

Fluoride nanoparticles show PL and RL. There were no distorted sites of Mn observed in the $\mathrm{Mn}^{2+}$ doped $\mathrm{BaMgF}_{3}$ as it was performed for the $\mathrm{NaMgF}_{3}$ nanoparticles and bulk materials. $\mathrm{Eu}^{3+}$ emission was detected in the RL spectra for the fluoride nanoparticles, which is different to the RL emission of $\mathrm{Eu}^{2+}$ and $\mathrm{Eu}^{3+}$ in $\mathrm{NaMgF}_{3}$ and $\mathrm{RbMgF}_{3}$ nanoparticles, which was reported in the previous chapters. The PL lifetimes for the $\mathrm{LaF}_{3}$ decreases with increasing rare earth concentrations that can be attributed to energy transfer from luminescence ions in the core to luminescence ion near the surface followed by non-radiative decay. In comparison with $\mathrm{RbMgF}_{3}$ and $\mathrm{NaMgF}_{3}$, the decrease of the PL lifetimes was more due to non-radiative recombination centres inside the crystal.

The RL spectrum for $\mathrm{LaF}_{3}$ shows only $\mathrm{Eu}^{3+}$ and $\mathrm{Sm}^{3+}$ occur. The integrated RL intensity is independent of dose for up to 5 orders of magnitude and for doses as high as $10 \mathrm{kGy}$. In 
the range of $\sim 100$ Gy to $\sim 10$ kGy the RL signal of the other fluoride nanoparticles is nearly independent of dose which are summarised in Tab. 9.3. The Eu doped $\mathrm{LaF}_{3}$ and $\mathrm{BaF}_{2}$ samples show that there have very little dose dependence of the RL signal. The $\mathrm{NaMgF}_{3}$ samples doped with $1 \%$ Eu showed a constant RL signal after receiving a pre-dose of 15 kGy and the $1 \% \mathrm{Mn}$ doped sample show a constant RL signal after a pre-dose of $\sim 1$ kGy.

Table 9.3: Summary of the dose dependence of the RL signal for different fluoride compounds after receiving a dose of $\sim 10 \mathrm{kGy}$.

\begin{tabular}{|l|l|}
\hline Compound & Dose dependence of RL (\%) \\
\hline \hline $\mathrm{LaF}_{3}: 5 \% \mathrm{Eu}$ & 3.0 \\
\hline $\mathrm{LaF}_{3}: 5 \% \mathrm{Sm}$ & 5.3 \\
\hline $\mathrm{BaF}_{2}: 5 \% \mathrm{Eu}$ & 1.3 \\
\hline $\mathrm{BaMgF}_{3}: 5 \% \mathrm{Mn}$ & 17.4 \\
\hline
\end{tabular}

The dose independence of the $\mathrm{RL}$ of $\mathrm{LaF}_{3}$ is a desirable property for real time radiation dosimeters that can detect low doses as well as very high doses without being damaged. Therefore $\mathrm{LaF}_{3}$ has potential as a dosimeter for non-tissue equivalent applications or radiation imaging materials. Further work should be performed on the dependency of the dose for the RL after longterm irradiation. PL measurements before and after heavy irradiation should also be performed to observe the change of the PL emission and the excitation spectra as a function of dose. 


\section{Summary and Conclusion}

In this thesis, fluoroperovskite materials have been investigated for their potential use in dosimetric applications. Both polycrystalline bulk materials and nanoparticles were researched and their behaviour in photoluminescence (PL), radioluminescence (RL), temperature dependent RL and thermoluminescence (TL) were compared. The main focus was on the RL and TL properties of these materials. These materials have the advantage that they can be employed as real-time dosimeters due to their good response to RL. The bulk materials and nanoparticles can be produced transparent, and therefore the incident light will not be scattered during stimulation of the sample. The nanoparticles are radiation hard for high doses. This means the RL is dose independent after heavy irradiation. $\mathrm{NaMgF}_{3}$ doped with $\mathrm{Mn}$ is nearly tissue equivalent, which means that the material responds to radiation in a similar way as tissue. This is a desirable property of dosimeters employed in personal dosimetry.

It was observed that the bulk materials show optically stimulated luminescence (OSL) in addition to RL and TL [3]. Therefore, their use would be for OSL dosimeters, because they show a high sensitivity in PL, TL, RL and OSL. Their RL is dose history dependent and hence not favourable in real-time applications. With Mn being the best material for dosimeter applications for the polycrystalline samples because it shows less dose history dependence. Their thermal coefficient of the RL was smaller than observed for other materials. The Eu doped bulk materials show additional unidentified defects at around $470 \mathrm{~nm}$. It shows a large PL lifetime for all of the different compounds. Hence, it can be assumed that the unknown defect is the same for the $\mathrm{Rb}$ and $\mathrm{Na}$ samples and needs to be 
further investigated. Therefore, further work should be performed to find the origin of the unknown peak and distinguish if it originates is a result of F-centres or other defects. One possibility could be, to measure the sample with EPR. If the unknown peak is dominated by F-centres, it would be possible to detect this with EPR [98]. Another possibility would be, to powder the sample and heat it up. The mean free path will increase and oxygen can diffuse inside the crystal. By performing PL after this, an increase of the peak would mean the defects in the peak are caused by oxygen. Oxygen can also be detected by performing vacuum UV-PL on the peak. Future work should also be performed to measure TL after heavy irradiation for the $\mathrm{NaMgF}_{3}$ : Eu and $\mathrm{RbMgF}_{3}$ samples.

The fluoroperovskite nanoparticles were produced with the reverse micro-emulsion method [76]. The nanoparticles can be pressed into pellets, which can be made transparent as it was shown for the $\mathrm{LaF}_{3}$ nanoparticles [22] depending on the thickness of the pressed sample and if they are capped with oleic acid. The $\mathrm{NaMgF}_{3}$ and $\mathrm{RbMgF}_{3}$ fluoroperovskite nanoparticles, which were observed in this thesis, were not capped with any organics and hence, their appearance was not transparent. None of the nanoparticles show any OSL. The generation of defects is less compared to the bulk materials. Therefore, the RL is less dependent on the dose. After a pre-dose of a few kGy, the nanoparticles with a larger particle size show a constant RL signal with increasing dose. This is a desirable property for real-time radiation dosimeters that can detect high doses without being damaged. Hence, the nanoparticles are suitable for applications as a real-time dosimeter. Mn or Eu doped nanoparticles are favourable for this application since both dopants show a dose history independent RL. However, a dosimeter with two detectors, Mn and Eu, can be produced with the nanoparticles. The thermal coefficient of the RL for the nanoparticles has a high uncertainty.

The Mn and Eu doped nanoparticles show a different trap distribution compared to the bulk materials, which is shown in the lower PL lifetimes. The bulk materials show radiation damage and trapping of charged carriers in non-radiative recombination centres centres and the nanoparticles show an energy transfer to the surface of the crystal. The 
crystal environment of the $\mathrm{Eu}^{3+}$ in the nanoparticles is less distorted compared to the bulk materials, which results in a lower ratio of $I_{F_{F}}: I_{7}$. The TL glow curves of $\mathrm{RbMgF}_{3}: \mathrm{Mn}$ and $\mathrm{NaMgF}_{3}$ : Mn bulk materials all show high temperature peaks, whereas the $\mathrm{RbMgF}_{3}$ : $\mathrm{Mn}$ and $\mathrm{NaMgF}_{3}$ : Mn nanoparticles show low temperature peaks. It was mentioned that for the determination of the thermal coefficient of the RL, deep traps might not be completely emptied after heating the sample up to $620 \mathrm{~K}$. Hence, further measurements should be performed with optical bleaching after each RL measurement.

For the fluoride nanoparticles, it was observed that the PL lifetimes for the $\mathrm{LaF}_{3}$ decreases with increasing rare earth concentrations. This can be attributed to energy transfer from luminescence ions in the core to luminescence ions near the surface followed by non-radiative decay. In comparison, the decrease of the PL lifetimes of $\mathrm{RbMgF}_{3}$ and $\mathrm{NaMgF}_{3}$ is predominantly due to non-radiative recombination centres inside the crystal. The RL spectra for $\mathrm{LaF}_{3}$ shows that only $\mathrm{Eu}^{3+}$ and $\mathrm{Sm}^{3+}$ was detected. The integrated RL intensity is independent of the dose for up to 5 orders of magnitude and for doses as high as $10 \mathrm{kGy}$. Therefore, $\mathrm{LaF}_{3}$ can be employed as a real-time dosimeter that can detect low doses as well as high doses without being damaged for non-tissue equivalent applications or radiation imaging materials. Further work should be performed on the dependency of the dose for the RL after long-term irradiation. PL measurements before and after heavy irradiation should also be performed to observe the change of the PL emission and the excitation spectra as a function of dose. 


\section{Bibliography}

[1] A. S. Beddart, T. R. Mackie, and F. H. Attix, "Water-equivalent plastic scintillation detectors for high- energy beam dosimetry," Phys. Med. Biol, vol. 37, no. 10, pp. 1883-1900, 1992.

[2] J. Seuntjens, W. Strydom, and K. Shortt, "Dosimetric principles, quantities and units," Radiation oncology physics, pp. 45-70, 2005.

[3] C. J. Dotzler, Optically Stimulated Luminescence in Fluoroperovskites for Dosimetric Applications. PhD thesis, Victoria University of Wellington, 2008.

[4] C. Dotzler, G. V. M. Williams, U. Rieser, and J. Robinson, "Photoluminescence, optically stimulated luminescence, and thermoluminescence study of $\mathrm{RbMgF}_{3}: \mathrm{Eu}^{2+}, "$ Journal of Applied Physics, vol. 105, no. 2, p. 023107, 2009.

[5] S. McKeever, M. Moscovitch, and P. Townsend, Thermoluminescence Dosimetry Materials: Properties and Uses. Nuclear Technology Publishing, 1995.

[6] L. Bøtter-Jensen, S. McKeever, and A. Wintle, Optically Stimulated Luminescence Dosimetry. Elvsevier, 2003.

[7] Varian, "Cary Eclipse Fluorescence Spectrophotometer.".

[8] Jobin-Yvon, "Jobin-Yvon FluoroLog ${ }^{\circledR}$ Spectrophotometer Handbook.". 
[9] E. Valyashko, S. Bodrug, V. N. Mednikova, and V. A. Smirnov, "Spectra of Eu" in $\mathrm{KMgF}_{3}$ and $\mathrm{NaMgF}_{3}$ crystals," Journal of Applied Physics, vol. 26, no. 5, pp. 628$633,1977$.

[10] G. Williams, S. Janssens, C. Gaedtke, and D. Clarke, "Observation of photoluminescence and radioluminescence in $\mathrm{Eu}$ and $\mathrm{Mn}$ doped $\mathrm{NaMgF}_{3}$ nanoparticles," Journal of Luminescence, 2013.

[11] C. Gaedtke, G. Williams, S. Janssens, S. Raymond, and D. Clarke, "Photoluminescence and radioluminescence study of Eu and $\mathrm{Mn}$ doped $\mathrm{RbMgF}_{3}$ nanoparticles," Radiation Measurements, pp. 4-8, Feb. 2013.

[12] C. Gaedtke, G. Williams, S. Janssens, S. Raymond, and D. Clarke, "The effect of ionizing radiation on the luminescence properties of $\mathrm{Eu}^{3+}$ and $\mathrm{Sm}^{3+}$ doped $\mathrm{LaF}_{3}$ nanoparticles," Physica Status Solidi (C), vol. 9, pp. 2247-2250, Dec. 2012.

[13] L. Zhang, M. Peng, G. Dong, and J. Qiu, "Spectroscopic properties of $\mathrm{Sm}^{3+}$-doped phosphate glasses," Journal of Materials Research, vol. 27, pp. 2111-2115, June 2012.

[14] L. Dussau and J. Gasiot, "Online and realtime dosimetry using optically stimulated luminescence," International Journal of High Speed Electronics and Systems, vol. 14, no. 02 , pp. 605-623, 2004.

[15] J. T. Bushberg, "Radiation Exposure and Contamination," 2009.

[16] Health Physics Society, "Radiation Exposure from Medical Diagnostic Imaging Procedures," pp. 1-3.

[17] B. F. Wall and D. Hart, "Revised radiation doses for typical X-ray examinations," The British Journal of Radiology, vol. 70, pp. 437-439, 1997.

[18] K. N. Shinde, S. Dhoble, H. Swart, and K. Park, Phosphate Phosphors for SolidState Lighting, vol. 174 of Springer Series in Materials Science. Berlin, Heidelberg: Springer Berlin Heidelberg, 2012. 
[19] S. Janssens, New Materials for Ultrafast Switching and Optical Regeneration. PhD thesis, Victoria University of Wellington, 2012.

[20] B. Walsh, Judd-Ofelt theory: principles and practices. Springer, 2006.

[21] R. Dekker, V. Sudarsan, F. Veggel, K. Wörhoff, and A. Driessen, "Erbium doped $\mathrm{LaF}_{3}$ nanoparticles incorporated in silicondioxide thin films for active integrated optical applications," Proceedings Symposium IEEE/LEOS Benelux Chapter, no. 1, pp. 3-6, 2004.

[22] S. Janssens, G. Williams, and D. Clarke, "Systematic study of sensitized $\mathrm{LaF}_{3}: \mathrm{Eu}^{3+}$ nanoparticles," Journal of Applied Physics, vol. 109, no. 023506, pp. 1-9, 2011.

[23] C. D’Ambrosio, F. de Notaristefani, G. Hull, V. Orsolini Cencelli, and R. Pani, "Study of $\mathrm{LaCl}_{3}$ :Ce light yield proportionality with a hybrid photomultiplier tube," Nuclear Instruments and Methods in Physics Research Section A: Accelerators, Spectrometers, Detectors and Associated Equipment, vol. 556, pp. 187-191, Jan. 2006.

[24] L. G. Jacobsohn, C. L. McPherson, K. B. Sprinkle, E. G. Yukihara, and T. A. Devol, "Scintillation of rare earth doped fluoride nanoparticles," Appl. Phys. Lett., vol. 99, p. 113111, 2011.

[25] Univeristy of Wisconsin and Madison, Radiation Detection and Measurement. 2005.

[26] G. Williams, "OSL Dosimetry." presentation at Wellington Hospital, Dec 2010.

[27] S. McKeever and M. Moscovitch, "Topics under debate: On the advantages and disadvantages of optically stimulated luminescence dosimetry and thermoluminescence dosimetry," Radiation protection dosimetry, vol. 104, no. 3, pp. 263-270, 2003.

[28] “§20.1004 units of radiation dose.” U.S. Nuclear Regulatory Commission, 2013.

[29] C. Dotzler, G. V. M. Williams, U. Rieser, and A. Edgar, "Optically stimulated luminescence in $\mathrm{NaMgF}_{3}: \mathrm{Eu}^{2+}$," Applied Physics Letters, vol. 91, no. 12, p. 121910, 2007. 
[30] Luxel+ and Track Etch Technology, "Luxel@+ Dosimeter for X-, Gamma-, Beta-, and Neutron Radiation," 2005.

[31] M. Akselrod, L. Bøtter-Jensen, and S. McKeever, "Optically stimulated luminescence and its use in medical dosimetry," Radiation measurements, vol. 41, pp. 78-99, 2006.

[32] E. Yukihara and S. McKeever, "Optically stimulated luminescence (OSL) dosimetry in medicine," Physics in medicine and biology, vol. 351, 2008.

[33] A. Macovski, Medical Imaging Systems. Englewood Cliffs, New York, 1983.

[34] E. C. McCullough, "Photon attenuation in computed tomography," Med. Phys., vol. 2, pp. 307-320, 1975.

[35] C. Furetta, M. Prokic, and R. Salamon, "Dosimetric characteristics of tissue equivalent thermoluminescent solid TL detectors based on lithium borate," Nuclear Instruments and Methods in Physics Research, vol. 456, no. A, pp. 411-417, 2001.

[36] K. Sankar, J. Plumley, T. Akins, B.A.and Memon, N. Withers, G. Smolyakov, and M. Osinski, "Synthesis and characterization of scintillating cerium-doped lanthanum fluoride nanocrystals," Proc. SPIE, vol. 7189, p. 718909, 2009.

[37] International Commission on Radiation units and Measurements, "ICRU report 51: Quantities and Units in radiation Protection Dosimetry," 1993.

[38] ICRU, "Fundamental Quantities and Units for Ionizing Radiation," ICRU report, vol. 60 .

[39] A. Pradhan, "Photon energy response of luminescence dosemeters and its impact on assessment of $\mathrm{Hp}(10)$ and $\mathrm{Hp}(0.07)$ in mixed fields of varying energies of photons and beta radiation.," Radiation protection dosimetry, vol. 101, pp. 173-8, Jan. 2002.

[40] S. Miljanic, B. Vekic, and R. Martincic, "Determination of X ray effective energy and absorbed dose using $\mathrm{CaF}_{2}$ : $\mathrm{Mn}$ and $\mathrm{LiF}$ : $\mathrm{Mg}$, Ti thermoluminescence dosemeters," Radiation protection dosimetry, vol. 85, pp. 381-384, 1999. 
[41] K. Becker, Solid State Dosimetry. CRC Press, Cleveland, OH, 1973.

[42] "Annals of the icrp," vol. 26, pp. 1-205, 1996.

[43] "5. Dosimetry," Journal of the ICRU, vol. 11, pp. 23-28, Apr. 2011.

[44] C. Wernli, "External dosimetry: operational quantities and their measurement," 11th International Congress of IRPA Madrid, no. May, 2004.

[45] G. Dietze, C. Streffer, C. M. W. Alberts, M. Balonov, V. Berkovski, A. Bouville, A. Edwards, J. Harrison, J. Lipzstein, M. Pellicioni, A. Phipps, and A. Pradhan, "Basis for dosimetric quantities used in radiological protection: Task Group of ICRP Committee 2 :," 2005.

[46] J. Zoetelief, H. Julius, and P. Christensen, Recommendations for patient dosimetry in diagnostic radiology using TLD. Topical report. EUR-19604, 2000.

[47] J. C. McDonald, "What is radiation protection dosimetry?," Radiation protection dosimetry, vol. 138, pp. 1-2, Jan. 2010.

[48] C. E. Andersen, M. C. Aznar, L. Bøtter-Jensen, S. Back, S. Mattsson, and J. Medin, "Development of optical fiber luminescence techniques for real time in vivo dosimetry in radiotherapy," Proceedings of the Standards and codes of practice in medical radiation dosimetry. International symposium in Vienna (AT), 25-28 November 2002, vol. 2, pp. 353-360, 2003.

[49] V. Rybka, L. Davydov, I. Shlyakhov, V. Kutny, I. Prokhoretz, D. Kutny, and a.N. Orobinsky, "Gamma-radiation dosimetry with semiconductor CdTe and CdZnTe detectors," Nuclear Instruments and Methods in Physics Research Section A: Accelerators, Spectrometers, Detectors and Associated Equipment, vol. 531, pp. 147-156, Sept. 2004.

[50] S. Beddar, M. Salehpour, T. M. Briere, H. Hamidian, and M. T. Gillin, "Preliminary evaluation of implantable MOSFET radiation dosimeters," Physics in Medicine and Biology, vol. 50, pp. 141-149, Jan. 2005. 
[51] B. Nilsson, B. Ruden, and B. Sorcini, "Characteristics of silicon diodes as patient dosimeters in external radiation therapy," Radiotherapy and Oncology, vol. 11(3), pp. 279-288, 1988.

[52] R. Gaza, S. McKeever, M. Akselrod, a. Akselrod, T. Underwood, C. Yoder, C. Andersen, M. Aznar, C. Marckmann, and L. Bøtter-Jensen, "A fiber-dosimetry method based on OSL from $\mathrm{Al}_{2} \mathrm{O}_{3}$ :C for radiotherapy applications," Radiation Measurements, vol. 38, pp. 809-812, Aug. 2004.

[53] G. D. Planque and T. Gesell, "Environmental measurements with thermoluminescence dosemeters-trends and issues," Radiation Protection Dosimetry, vol. 17, pp. 193-200, 1986.

[54] G. Klemic, J. Shobe, S. Sengupta, and P. Shebell, "State of the art of environmental dosimetry: 11th international intercomparison and proposed performance tests," Radiation Protection Dosimetry, vol. 85, pp. 201-206, 1999.

[55] S. W. S. McKeever, M. W. Blair, E. Bulur, R. Gaza, R. Gaza, R. Kalchgruber, D. M. Klein, and E. G. Yukihara, "Recent advances in dosimetry using the optically stimulated luminescence of $\mathrm{Al}_{2} \mathrm{O}_{3}$ :C.," Radiation protection dosimetry, vol. 109, pp. 269-76, Jan. 2004.

[56] E. I. Tolstykh, M. O. Degteva, E. a. Shishkina, V. I. Zalyapin, and V. a. Krivoschapov, "Possibilities of using human teeth for retrospective dosimetry: analysis of the Techa River data.," Radiation protection dosimetry, vol. 127, pp. 511-5, Jan. 2007.

[57] B. Henke, Structure Sensitive Investigations on Mn-activated Perovskites and RareEarth-doped Aluminates. PhD thesis, University of Paderborn, 2007.

[58] K. Gschneidner Jr, L. Eyring, and G. Lander, Handbook on the physics and chemistry of rare earths. Elsevier Science B.V., vol. 25 ed., 1998. 
[59] J. Garcia, W. Sibley, C. Hunt, and J. Spaeth, "Optical properties of $\mathrm{Eu}^{2+}$, $\mathrm{Eu}^{3+}$ and $\mathrm{Mn}^{2+}$ in cubic perovskite crystals ," Journal of Luminescence, vol. 42, no. 1, pp. 35-42, 1988.

[60] Honbun, "Judd-Ofelt analysis of RE-M nanoclusters," pp. 111-134.

[61] M. J. Weber, J. D. Myers, and D. H. Blackburn, "Optical properties of $\mathrm{Nd}^{3+}$ in tellurite and phosphotellurite glasses," Journal of Applied Physics, vol. 52, no. 4, p. 2944, 1981.

[62] R. Sosa, M. Flores, T. Rodrìguez, and A. Munoz, "Optical properties Judd-Ofelt intensity parameters of $\mathrm{Eu}^{3+}$ in PMMA: PAAC copolymer samples," Rev. Mex. Fis., vol. 49, no. 6, pp. 519-524, 2009.

[63] G. de Sà, O. Malta, C. de Mello Donegà, A. Simas, R. Longo, P. Santa-Cruz, and E. da Silva Jr., "Spectroscopic properties and design of highly luminescent lanthanide coordination complexes," Coordination Chemistry Reviews, vol. 196, pp. 165-195, 2000.

[64] J. Kai, D. F. Parra, and H. F. Brito, "Polymer matrix sensitizing effect on photoluminescence properties of $\mathrm{Eu}^{3+}-\beta$-diketonate complex doped into poly- $\beta$ hydroxybutyrate (PHB) in film form," Journal of Materials Chemistry, vol. 18, no. 38, p. 4549, 2008.

[65] W. Krupke, "Induced-emission cross sections in neodymium laser glasses," IEEE Journal of Quantum Electronics, no. 4, pp. 450-457, 1974.

[66] W. T. Carnall, G. L. Goodman, K. Rajnak, and R. S. Rana, "A systematic analysis of the spectra of the lanthanides doped into single crystal $\mathrm{LaF}_{3}$," The Journal of Chemical Physics, vol. 90, no. 7, p. 3443, 1989.

[67] A. Meijerink, "Spectroscopy and Vibronic Transitions of Divalent Europium in $\mathrm{LiBaF}_{3}$," Journal of Luminescence, vol. 55, pp. 125-138, 1993.

[68] M. Weber, Optical Properties of Ions in Crystal. Interscience, 1967. 
[69] R. Meltzer, W. Yen, and H. Zheng, "Effect of the matrix on the radiative lifetimes of rare earth doped nanoparticles embedded in matrices," Journal of Luminescence, vol. 95, pp. 217-220, 2001.

[70] G. Williams, S. Raymond, N. Denton, and C. Gaedtke, "Development and Characterization of a Portable Fibre Optic Dosimeter," MATERIALS SCIENCE FORUM, vol. 700 , pp. $174-177,2012$.

[71] G. Williams and S. Raymond, "Fiber-optic-coupled $\mathrm{RbMgF}_{3}: \mathrm{Eu}^{2+}$ for remote radiation dosimetry," Radiation Measurements, vol. 46, pp. 1099-1102, 2011.

[72] P. Dorenbos, C. van Eijk, R. Hollander, and P. Schotanus, "Scintillation properties of $\mathrm{Nd}^{3+}$ doped $\mathrm{LaF}_{3}$ crystals," IEEE TRANSACTIONS ON NUCLEAR SCIENCE, vol. 37, no. 2, pp. 119-123, 1990.

[73] J. Polf, E. Yukihara, M. Akselrod, and S. McKeever, "Real-time luminescence from $\mathrm{Al}_{2} \mathrm{O}_{3}$ fiber dosimeters," Radiation Measurements, vol. 38, pp. 227-240, Apr. 2004.

[74] R. Chen and P. L. Leung, "Dose dependence and dose-rate dependence of the optically stimulated luminescence signal," Journal of Applied Physics, vol. 89, no. 1, p. 259, 2001.

[75] W. Pingqiang, C. Zhaoyang, F. Yanwei, S. Yurun, and Z. Yun, "Dose-rate dependence of optically stimulated luminescence signal," Journal of Semiconductors, vol. 31, p. 102001, Oct. 2010.

[76] R. Hua, Z. Jia, and C. Shi, "Preparation of $\mathrm{KMgF}_{3}$ and Eu-doped $\mathrm{KMgF}_{3}$ nanocrystals in water-in-oil microemulsions," Materials research bulletin, vol. 42, pp. 249-255, 2007.

[77] S. Janssens, G. Williams, and D. Clarke, "Synthesis and characterization of rare earth and transition metal doped $\mathrm{BaMgF}_{4}$ nanoparticles," Journal of Luminescence, vol. 134, pp. 277-283, Feb. 2013. 
[78] A. Guinier, X-ray diffraction: In crystals, imperfect crystals and amorphous bodies. Dover Publications, 1994.

[79] T. Nishimatsu, N. Terakubo, H. Mizuseki, Y. Kawazoe, D. a. Pawlak, K. Shimamura, and T. Fukuda, "Band Structures of Perovskite-Like Fluorides for VacuumUltraviolet-Transparent Lens Materials," Japanese Journal of Applied Physics, vol. 41, pp. L365-L367, Apr. 2002.

[80] N. Kristianpoller, D. Weiss, and R. Chen, "Radiation effects in $\mathrm{KMgF}_{3}$ crystals," Radiation effects and defects in Solids, vol. 157, pp. 583-588, Jan. 2002.

[81] M. True, Fine structure in $d-f$ and $f$ - $f$ transitions of $\mathrm{Tm}^{3+}$ and systematic investigations of $3 d^{5}-3 d^{4} 4 s$ absorption of $\mathrm{Mn}^{2+}$ doped fluorides. $\mathrm{PhD}$ thesis, Hamburg University, 2004.

[82] R. Alcala, D. K. Sardar, and W. Sibley, "Optical transitions of $\mathrm{Eu}^{2+}$ ions in $\mathrm{RbMgF}_{3}$ crystals," Journal of Luminescence, vol. 27, pp. 273-284, 1982.

[83] V. Sudarsan, F. C. J. M. van Veggel, R. a. Herring, and M. Raudsepp, "Surface Eu" ions are different than "bulk" $\mathrm{Eu}^{3+}$ ions in crystalline doped $\mathrm{LaF}_{3}$ nanoparticles," Journal of Materials Chemistry, vol. 15, no. 13, p. 1332, 2005.

[84] M. Dejneka, E. Snitzer, and R. Riman, "Blue, green and red fluorescence and energy transfer of $\mathrm{Eu}^{3+}$ in fluoride glasses," Journal of Luminescence, vol. 65, pp. 227-245, 1995.

[85] J. Choi, T.-K. Tseng, M. Davidson, and P. H. Holloway, "Enhanced photoluminescence from $\mathrm{Gd}_{2} \mathrm{O}_{3}: \mathrm{Eu}^{3+}$ nanocores with a $\mathrm{Y}_{2} \mathrm{O}_{3}$ thin shell," Journal of Materials Chemistry, vol. 21, no. 9, p. 3113, 2011.

[86] L. G. Jacobsohn, a. Toncelli, K. B. Sprinkle, C. J. Kucera, and J. Ballato, "Spectral engineering of $\mathrm{LaF}_{3}: \mathrm{Ce}^{3+}$ nanoparticles: The role of $\mathrm{Ce}^{3+}$ in surface sites," Journal of Applied Physics, vol. 111, no. 7, p. 074315, 2012. 
[87] K. Baumann, "On the Temperature Dependence of the Band Gap in Semiconductors," physica status solidi (b), vol. 71, pp. 8-11, 1974.

[88] H. Cardona, M. Lauck, and R. K. Kremer, "Temperature dependence of band gaps in semiconductors: Electron-phonon interaction," Physical Review B, vol. 86, p. 195208, Nov. 2012.

[89] S. Kazeminia, K. Hadidi, A. Khoei, and M.-N. Azarmanesh, "Effect of bandgap energy temperature dependence on thermal coefficient of bandgap reference voltage," 2011 20th European Conference on Circuit Theory and Design (ECCTD), pp. 449452, Aug. 2011.

[90] K. P. O'Donnell and X. Chen, "Temperature dependence of semiconductor band gaps," Applied Physics Letters, vol. 58, no. 25, p. 2924, 1991.

[91] D. L. Dexter, "A Theory of Sensitized Luminescence in Solids," The Journal of Chemical Physics, vol. 21, no. 5, p. 836, 1953.

[92] A. Souza, L. Rodrigues, and H. Brito, "Novel europium and gadolinium complexes with methaneseleninate as ligand: synthesis, characterization and spectroscopic study," Inorganic Chemistry Communications, vol. 15, pp. 97-101, 2012.

[93] H. Brito, O. Malta, and J. Menezes, "Luminescent properties of diketonates of trivalent europium with dimethyl sulfoxide," Journal of Alloys and Compounds, vol. 303304, pp. 336-339, May 2000.

[94] L. Liu and X. Chen, "Energy levels, fluorescence lifetime and Judd-Ofelt parameters of $\mathrm{Eu}^{3+}$ in $\mathrm{Gd}_{2} \mathrm{O}_{3}$ nanocrystals," Nanotechnology, vol. 255704, 2007.

[95] K. Nissamudeen and K. Gopchandran, " $\mathrm{Y}_{2} \mathrm{O}_{3}: \mathrm{Eu}^{3+}$ based nanophosphors with higher oscillator strength through lithium incorporation and indirect oxidation," Journal of Alloys and Compounds, vol. 490, pp. 399-406, Feb. 2010. 
[96] C. J. Dotzler, G. V. M. Williams, and a. Edgar, "Thermoluminescence, photoluminescence and optically stimulated luminescence properties of X-ray irradiated $\mathrm{RbMgF}_{3}: \mathrm{Mn}^{2+}, "$ Physica Status Solidi (C), vol. 4, pp. 992-995, Mar. 2007.

[97] C. Dotzler, G. Williams, and A. Edgar, "Radiation-induced optically and thermally stimulated luminescence in $\mathrm{RbCdF}_{3}$ and $\mathrm{RbMgF}_{3}, "$ Current Applied Physics, vol. 8, pp. 447-450, 2008.

[98] C. Dotzler, G. V. M. Williams, a. Edgar, S. Schweizer, B. Henke, J. M. Spaeth, a. Bittar, J. Hamlin, and C. Dunford, "The effect of x-ray, $\gamma$-ray, and UV radiations on the optical properties of $\mathrm{RbCdF}_{3}: \mathrm{Mn}^{2+}, "$ Journal of Applied Physics, vol. 100, no. 3, p. 033102, 2006.

[99] S. Janssens, G. Williams, D. Clarke, and M. Sharp, "Photoluminescence study of $\mathrm{LaF}_{3}: \mathrm{Eu}^{3+}$ nanoparticles capped with different ligands," physica status solidi (b), vol. 443, no. 2, pp. 439-443, 2011.

[100] S. Schweizer, L. Hobbs, M. Secu, J.-M. Spaeth, A. Edgar, G. Williams, and J. Hamlin, "Photostimulated luminescence from fluorochlorozirconate glass ceramics and the effect of crystallite size," Journal of Applied Physics, vol. 97, no. 8, p. 083522, 2005.

[101] G. Williams, A. Bittar, C. Dotzler, A. Beaudin, C. Varoy, and C. Dunford, "Glassceramics and epoxy-composites for radiation imaging," Radiation Measurements, vol. 42, pp. 899-902, Apr. 2007.

[102] J. Wang, J. Hu, D. Tang, X. Liu, and Z. Zhen, "Oleic acid (OA)-modified LaF 3 :Er,Yb nanocrystals and their polymer hybrid materials for potential optical-amplification applications," Journal of Materials Chemistry, vol. 17, no. 16, p. 1597, 2007.

[103] M. Werts, R. Jukes, and J. Verhoeven, "The emission spectrum and the radiative lifetime of $\mathrm{Eu}^{3+}$ in luminescent lanthanide complexes," Physical Chemistry Chemical Physics, vol. 4, no. 9, pp. 1542-1548, 2002. 
[104] S. Janssens, G. Williams, and D. Clarke, "Samarium Doped KMgF $\mathrm{F}_{3}$ Nanoparticles," Materials Science Forum, vol. 700, pp. 45-48, Sept. 2011.

[105] P. Solarz and W. Ryba-Romanowski, "Luminescence and energy transfer processes of $\mathrm{Sm}^{3+}$ in $\mathrm{K}_{5} \mathrm{Li}_{2} \mathrm{LaF}_{10}: \mathrm{Sm}^{3+}-\mathrm{K}_{5} \mathrm{Li}_{2} \mathrm{SmF}_{10}$ single crystals," Physical Review B, vol. 72 , p. 075105 , Aug. 2005. 


\section{List of publications}

1. C. Gaedtke, G.V.M. Williams, "Photoluminescence and radioluminescence study of $\mathrm{NaMgF}_{3}:$ Eu nanoparticles", Radiation Measurements, in press.

2. C. Gaedtke, G.V.M. Williams, D. Clarke, "Thermoluminescence studies of nanopar-

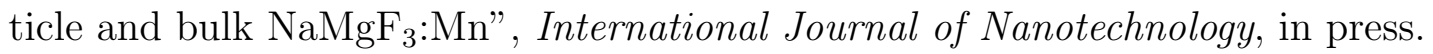

3. C. Gaedtke, G.V.M. Williams, S. Janssens, S. Raymond, D. Clarke, "Photoluminescence and radioluminescence study of $\mathrm{Eu}$ and $\mathrm{Mn}$ doped $\mathrm{RbMgF}_{3}$ nanoparticles", Radiation Measurements 56, 187-191, (2013).

4. G.V.M. Williams, S. Janssens, C. Gaedtke, S.G. Raymond, D. Clarke, "Observation of photoluminescence and radioluminescence in $\mathrm{Eu}$ and $\mathrm{Mn}$ doped $\mathrm{NaMgF}_{3}$ nanoparticles", Journal of Luminescence, 143, 219-225 (2013).

5. C. Gaedtke, G. V. M. Williams, S. Janssens, S. G. Raymond, D. J. Clarke, "The effect of ionizing radiation on the luminescence properties of $\mathrm{Eu}^{3+}$ and $\mathrm{Sm}^{3+}$ doped $\mathrm{LaF}_{3}$ nanoparticles", Phys. Status Solidi (c) 9, No. 12, 2247-2250 (2012).

6. G.V.M. Williams, S.G. Raymond, N. Denton, C. Gaedtke, "Development and characterization of a portable fibre optic dosimeter", Materials Science Forum 700, 174 (2011). 